Universidade de São Paulo

Instituto de Física

\title{
Aspectos de Teoria de Campos e Mecânica Estatística
}

\author{
Pedro Rogério Sergi Gomes \\ ORIENTADOR: Prof. Dr. Marcelo Otavio Caminha Gomes
}

Tese apresentada ao Instituto de Física da Universidade de São Paulo para obtenção do título de Doutor em Ciências

Comissão Examinadora

Prof. Dr. Marcelo Otavio Caminha Gomes (IFUSP)

Prof. Dr. Fernando Tadeu Caldeira Brandt (IFUSP)

Prof. Dr. Silvio Roberto de Azevedo Salinas (IFUSP)

Prof. Dr. Eduardo Cantera Marino (UFRJ)

Prof. Dr. Francisco Castilho Alcaraz (IFSC/USP)

São Paulo

2013 
Theoretical Physics is truly blessed, in that the quests for truth and beauty coincide. Kerson Huang 


\section{Agradecimentos}

Ao longo da minha trajetória, tive a oportunidade de encontrar algumas pessoas das quais pude extrair muitas coisas boas. Gostaria de expressar aqui minha profunda gratidão a cada uma delas.

Ao meu orientador, o professor Marcelo Gomes, que é um excelente orientador e físico, dando a liberdade necessária para o meu desenvolvimento sem deixar perder o foco. Além disso, tem uma característica especial que aprecio: a de pensar bastante sobre as questões até que fiquem completamente esclarecidas; não simplesmente as esquece. Muitas foram as vezes que após uma questão que nos deparávamos em nossos estudos, ele aparecia na minha sala algum tempo depois para mostrar o que havia pensado. Acredito que essa é uma característica essencial em um cientista.

À minha mulher e companheira Paula Fernanda Bienzobaz, por ser a pessoa especial que é e com quem divido a minha vida com grande felicidade. Além disso, nossa convivência me proporcionou um contato maior com a Mecânica Estatística e influenciou não apenas este trabalho, mas também minhas atividades científicas.

Aos meus pais, Pedro Waldir Gomes e Maria Aparecida Sergi Gomes, e minha irmã Maria Carolina Sergi Gomes, por todo carinho, apoio e incentivo que sempre me deram.

Aos amigos Leandro Ibiapina Beviláqua e Renann Lipinski Jusinskas, meus principais incentivadores no estudo da Teoria de Cordas, que muito me influenciaram e com quem aprendi bastante. Em especial ao Renann pelas string sections, nas quais sempre posso esclarecer diversos pontos.

Aos professores da época da graduação em Maringá, Luiz Roberto Evangelista e Renio dos Santos Mendes, que foram muito importantes no início dos meus estudos, e por me apresentarem a física teórica sempre com um entusiasmo contagiante.

À Fapesp, não apenas pelo apoio financeiro, mas por ser uma excelente agência de financiamento à pesquisa, que deveria servir de exemplo para todas agências do Brasil. 


\section{Resumo}

A teoria quântica de campos pode ser vista como um conjunto de métodos e idéias que além de sua importância no estudo das partículas elementares, tem sido amplamente usada em outras áreas. Em especial, ela constitui uma ferramenta indispensável no estudo moderno de transições de fases e fenômenos críticos. A origem dessa constante relação entre a teoria de campos e a matéria condensada deve-se ao fato que, apesar de suas diferenças superficiais, ambas tratam de problemas envolvendo um grande número de

graus de liberdade. Assim, não é surpreendente que as mesmas técnicas possam ser úteis nos dois campos.

Este trabalho trata de problemas nessas duas áreas e está essencialmente divido em duas partes. A primeira parte é dedicada ao estudo de teorias de campos com uma anisotropia entre o espaço e o tempo, o que implica uma quebra da simetria de Lorentz. Uma das motivações para considerar esse tipo de teoria vem justamente do estudo de transições de fase em sistemas da matéria condensada. Análises do grupo de renormalização com ênfase na possibilidade de restauração da simetria de Lorentz e também uma discussão sobre identidades de Ward são realizadas. Na segunda parte, a atenção é voltada para a mecânica estatística mas com uma abordagem típica da teoria de campos, em especial, voltada para o estudo de transições de fase clássicas e quânticas a partir da versão quantizada do modelo esférico e de sua extensão supersimétrica. 


\section{Abstract}

Quantum field theory can be seen as a set of methods and ideas that, besides its importance in the study of the elementary particles, has been widely used in other areas. In particular, it constitutes an indispensable framework in the modern approach to phase transitions and critical phenomena. The origin of this constant relationship between field theory and condensed matter is due to the fact that despite their superficial differences, both deal with problems involving a large number of degrees of freedom. Thus, it is not surprising that the same techniques may be useful in both fields.

This work addresses problems in these two areas and it is essentially divided in two parts. The first part is devoted to the study of field theories with an anisotropy between space and time, which implies a breaking of the Lorentz symmetry. One of the motivations for considering this kind of theory is precisely the study of phase transitions in condensed matter systems. Renormalization group analysis with emphasis on the possibility of restoration of the Lorentz symmetry and also a discussion about Ward identities are performed. In the second part, the attention is centered on statistical mechanics but with an approach typical of field theory, in particular, focused to the study of classical and quantum phase transitions from the quantized version of the spherical model and its supersymmetric extension. 


\section{Sumário}

\begin{tabular}{lr}
\hline Introdução & 9
\end{tabular}

1 Aspectos Gerais 13

1.1 Teorias Relativísticas com Derivadas de Ordem Superior . . . . . . . . . . 13

1.2 Quebra Suave da Simetria de Lorentz . . . . . . . . . . . . . . . . . . 15

1.3 Transicões de Fase Contínuas $\ldots \ldots \ldots$. . . . . . . . . . . . . . . 18

1.4 Construcão de Modelos . . . . . . . . . . . . . . . . . . . . . . . . . . . . 22

1.4 .1 Campos Escalares e Espinorias . . . . . . . . . . . . . . . 22

1.4 .2 Teorias de Calibre. . . . . . . . . . . . . . . . . . . . . . . . 23

1.4 .3 Gravitação . . . . . . . . . . . . . . . . . . . . 25

2 Grupo de Renormalização - Teorias Escalares 27

2.1 Teoria Livre . . . . . . . . . . . . . . . . . . . . . . . . . . . 27

2.2 Renormalizacão . . . . . . . . . . . . . . . . . . . . . . . . . . . . . 28

2.3 O modelo $\varphi^{3}$ e Liberdade Assintótica . . . . . . . . . . . . . . . . . 30

2.4 Parte Divergente das Funções de Vértice . . . . . . . . . . . . . . . . . 30

2.5 Funcão de 2 pontos . . . . . . . . . . . . . . . . . . . . . . 33

2.6 Funcão de 3 pontos . . . . . . . . . . . . . . . . . . . . . . . . . . . 36

2.7 Equacão do Grupo de Renormalizacão … . . . . . . . . . . . . . 37

2.8 Funcões do Grupo de Renormalização . . . . . . . . . . . . . . . . . . . . . . . . 39

2.8 .1 Funcão de 2 pontos . . . . . . . . . . . . . . . . . . . . . . . . . 39

2.8 .2 Funcão de 3 pontos . . . . . . . . . . . . . . . . . . . . . 40

2.9 Solucão Perturbativa $\ldots \ldots \ldots$. . . . . . . . . . . . . . . . . . . . . . . . . . . . . 41

2.10 Modelo $\varphi^{4} \ldots \ldots \ldots \ldots$. . . . . . . . . . . . . . . . . . . 44

2.10 .1 Grupo de Renormalizacão . . . . . . . . . . . . . . . . 46

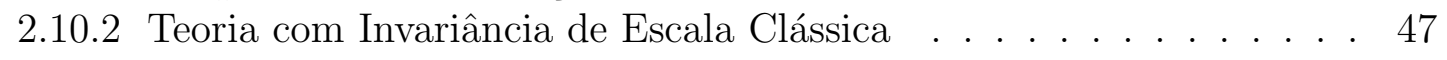

3 Grupo de Renormalizacão - Modelo de Yukawa 50

3.1 Consideracões Gerais e Contagem de Potências . . . . . . . . . . . . . 50

3.2 Modelo de Yukawa . . . . . . . . . . . . . . . . . . . . . . . . . 51

3.3 Parâmetros Efetivos . . . . . . . . . . . . . . . . . . . . . 54 
4 Identidades de Ward 59

4.1 Simetrias Clássicas . . . . . . . . . . . . . . . . . . . . . . . . . . 59

4.2 Simetrias Quânticas . . . . . . . . . . . . . . . . . . . . . . . 60

4.3 Identidades de Ward em Teorias com Anisotropia . . . . . . . . . . . . . . 62

4.3 .1 Produtos Normais. . . . . . . . . . . . . . . . . . . 62

4.4 Tensor de Energia-Momento . . . . . . . . . . . . . . . . . . . . . . . . . . . 69

4.5 Anomalia de Dilatacão . . . . . . . . . . . . . . . . . . . 70

4.5 .1 Análise Clássica . . . . . . . . . . . . . . . . . . . 70

4.5 .2 Análise Quântica . . . . . . . . . . . . . . . . . . 71

5 Modelos Estatísticos Clássicos $\quad 75$

5.1 Modelo de Ising . . . . . . . . . . . . . . . . . . . . . . . . 75

5.1 .1 Conexão com a Teoria de Campos . . . . . . . . . . . . . . . . . 80

5.2 Modelo Esférico Clássico . . . . . . . . . . . . . . . . . . . . 83

6 Modelo Esférico Quântico $\quad 87$

6.1 Transicões de Fase Quânticas . . . . . . . . . . . . . . . . . . . . . 87

6.2 Modelo Esférico Quântico . . . . . . . . . . . . . . . . . . . . . . . . . 88

6.2 .1 Formalismo do Tempo Imaginário . . . . . . . . . . . . . . . . 89

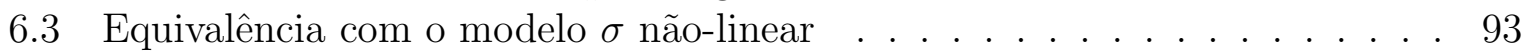

7 Modelo Esférico Quântico Supersimétrico 96

7.1 Idéia de Supersimetria . . . . . . . . . . . . . . . . . . . . . . . . . 96

7.2 Supersimetria e Transicões de Fase Quânticas . . . . . . . . . . . . . . . . 97

7.3 Extensão Supersimétrica $\ldots \ldots \ldots \ldots$. . . . . . . . . . . . . . . . . . . . . . . . 99

7.4 Funcão de Particão . . . . . . . . . . . . . . . . . . . . . . . . . . . . . . . 100

7.5 Comportamento Crítico . . . . . . . . . . . . . . . . . . . . . . 103

7.5 .1 Temperatura Finita . . . . . . . . . . . . . . . . . . 103

7.5 .2 Temperatura Zero . . . . . . . . . . . . . . . . . . 105

$\begin{array}{ll}\text { Considerações Finais } & 107\end{array}$

A Grupo de Renormalizacão - Modelo de Yukawa 110

A.1 Parte Divergente das Integrais . . . . . . . . . . . . . . . . . . . . . . . 110

A.1.1 Funcão de 2 pontos escalar . . . . . . . . . . . . . . . . . . . . 112

A.1.2 Funcão de 2 pontos espinorial . . . . . . . . . . . . . . . . . . . . 115

A.1.3 Funcão de 3 pontos . . . . . . . . . . . . . . . . . . . . . . . . . . . 118

A.1.4 Funcão de 4 pontos . . . . . . . . . . . . . . . . . . . . . . . . . . . 119

A.2 Grupo de Renormalizacão . . . . . . . . . . . . . . . . . . . . . . . . . . 119

A.2.1 Funcão de 2 pontos escalar . . . . . . . . . . . . . . . . . . . . . 120

A.2.2 Funcão de 2 pontos espinorial . . . . . . . . . . . . . . . . . 121

A.2.3 Função de 3 pontos . . . . . . . . . . . . . . . . . . . . . . . . . . 121 
A.2.4 Funcão de 4 pontos . . . . . . . . . . . . . . . . . . . . . . . . . 121

A.3 Solucão Perturbativa . . . . . . . . . . . . . . . . . . . . . . 122

A.3.1 Funcão de 2 pontos escalar . . . . . . . . . . . . . . . . . . . . . . . . 122

A.3.2 Funcão de 2 pontos espinorial . . . . . . . . . . . . . . . . . . . . . 122

A.3.3 Funcão de 3 pontos . . . . . . . . . . . . . . . . . . . . 123

A.3.4 Funcão de 4 pontos . . . . . . . . . . . . . . . . . . . . 123

A.4 Parâmetros Efetivos . . . . . . . . . . . . . . . . . 123

B Teorema de Noether para teorias com derivadas de terceira ordem 124

\begin{tabular}{lll}
\hline C Soma de Séries Infinitas via Teorema de Resíduos & 126
\end{tabular}

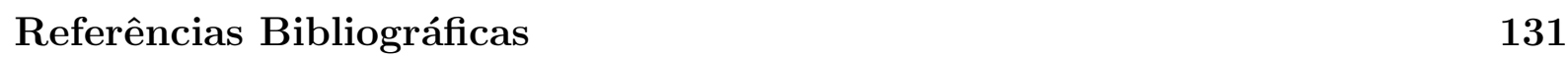




\section{Introdução}

A estrutura de curtas distâncias do espaço-tempo não é totalmente compreendida. Não sabemos ao certo quais são os graus de liberdade relevantes em tais escalas. Contudo, a descrição das partículas observadas e suas interações não depende desse entendimento. Isso porque a física, de uma maneira geral, é caracterizada pelo desacoplamento entre diferentes escalas de comprimento ou, equivalentemente, de energia. Em particular, na teoria de campos, o que ocorre em distâncias abaixo de uma certa escala, $\Lambda^{-1}$, não interfere na física em escalas superiores. $\Lambda$ é denominado corte (cutoff) da teoria e fisicamente pode ser interpretado como o seu regime de validade. Isso nos conduz à idéia de descrições efetivas.

Em teorias renormalizáveis, o corte $\Lambda$ pode ser eliminado completamente por meio de uma reparametrização dos parâmetros envolvidos (um conjunto finito), em contraste com teorias não-renormalizáveis, que são caracterizadas pela impossibilidade de eliminar $\Lambda$ dessa forma. O exemplo clássico e mais desafiador de uma teoria não-renormalizável é a gravitação [1].

Isso sugere que deve haver uma descrição mais fundamental, em que uma nova física deve governar tais regimes extremos. Nessa busca, a teoria de cordas aparece como uma candidata considerável, proporcionando alguns resultados impressionantes [2]. Apesar de haver muitos problemas pela frente, provavelmente ela contém alguns ingredientes fundamentais para uma descrição unificada da natureza.

Já que estamos imaginando uma situação em que uma nova física deve emergir, é natural questionar sobre o caráter dessas novas leis físicas. Por exemplo, quais as simetrias fundamentais nessa nova escala? Ou melhor, se as simetrias exibidas por um determinado processo (digamos, um espalhamento de partículas) sobrevivem à medida que se muda a escala em que o sistema é observado. Ou ainda, se novas simetrias possam surgir, como se acredita acontecer com a supersimetria. É difícil responder a essas questões, uma vez que escalas muito elevadas não são acessíveis experimentalmente. Essencialmente, os únicos guias que temos são a consistência da teoria subjacente e os limites de baixas energias.

Nesse sentido, o tema principal da primeira parte deste trabalho refere-se à possibilidade da quebra da simetria de Lorentz. Essa possibilidade já foi considerada em diversos trabalhos e ainda é motivo de intenso estudo. Veja as referências [3, 4, 5, 6, 7], por exemplo. Há diferentes maneiras para implementar essa quebra. De uma maneira geral, nos 
concentraremos na quebra da simetria de Lorentz por meio de uma modificação na parte livre da ação, em favor de uma melhora no comportamento ultravioleta da teoria. Mais precisamente, incluindo termos com derivadas espaciais de ordem superior. Dessa forma, o sistema passa a exibir uma anisotropia entre o espaço e o tempo.

Estudos no sentido de melhorar o comportamento ultravioleta adicionando-se termos com derivadas de ordem superior, mas preservando a simetria de Lorentz, já foram realizados e são muito discutidos atualmente [8, 9]. A covariância relativística requer também termos com derivadas temporais de ordem superior. Decorrente desses termos, surge o problema do aparecimento de fantasmas, comprometendo assim a unitariedade da teoria. No primeiro capítulo, há uma seção dedicada a ilustrar essa questão.

Devido a essas dificuldades, foi sugerido em [10, 11, 12, 13] a introdução de derivadas superiores de maneira anisotrópica, ou seja, apenas derivadas espaciais. Ao adicionar termos envolvendo apenas derivadas espaciais de ordem superior, não temos o problema de aparecimento de fantasmas e ainda assim temos a melhora no comportamento ultravioleta desejada. O prejuízo aqui deve-se à quebra da simetria de Lorentz, conforme já foi mencionado. A idéia geral é que a simetria de Lorentz possa emergir num limite de baixas energias. Um dos nossos objetivos é investigar, de um modo mais quantitativo, a viabilidade dessas idéias.

Esse tipo de comportamento anisotrópico entre espaço e tempo é comum em alguns sistemas de matéria condensada, no contexto de transições de fase e fenômenos críticos 14, 15, 16, 17]. Realmente, esse grau de anisotropia é caracterizado por um expoente crítico, denominado expoente crítico dinâmico $z$ e pode ser associado à existência de um ponto de Lifshitz quântico. Deixamos para uma seção posterior uma discussão um pouco mais elaborada desse aspecto.

O estudo de transições de fase e fenômenos críticos é uma das principais atividades da mecânica estatística e possui diversas conexões com a teoria de campos [18, 19, 20]. Como será discutido, nas proximidades do ponto crítico, uma rede discreta pode ser aproximada por um espaço contínuo e consequentemente o sistema passa a ser descrito efetivamente por uma teoria de campos. Como exemplos tradicionais de modelos definidos sobre redes cristalinas destacamos o modelo de Ising e o modelo esférico.

O modelo de Ising desempenha um papel fundamental no estudo de transições de fases em sistemas magnéticos. Apesar da simplicidade de sua formulação, o cálculo da função de partição se torna mais elaborado à medida que aumentamos a dimensãd1. De fato, em uma dimensão espacial o modelo é exatamente solúvel na presença de campo externo, mas não exibe uma transição de fase. Em duas dimensões, ele tem uma solução exata na ausência de campo (solução de Onsager) e em três dimensões não possui solução exata mesmo na ausência de campo.

Devido a essas dificuldades, em 1952 Berlin e Kac [21] introduziram o modelo esférico, que é uma espécie de aproximação contínua do modelo de Ising. A função de partição

\footnotetext{
${ }^{1}$ Até o máximo de três dimensões, uma vez que a partir de quatro dimensões o modelo se torna trivial, com um comportamento crítico de campo médio.
} 
do modelo esférico é exatamente solúvel em qualquer número de dimensões mesmo na presença de campo externo e a solução exibe um comportamento crítico não trivial, o que o torna um excelente laboratório para investigar diversas propriedades. Além disso, como mostrado por Stanley em 1968 [22], o modelo esférico é equivalente ao modelo de Heisenberg clássico no limite de spins com dimensionalidade infinita.

Apesar de suas características muito interessantes, o modelo apresenta uma patologia na entropia em baixas temperaturas: $S \sim \ln T$ e assim $S \rightarrow-\infty$ quando $T \rightarrow 0$, em contradição com a terceira lei da termodinâmica. Na tentativa de melhorar esse comportamento, versões quânticas do modelo esférico passaram a ser consideradas [23, 24]. Em sua versão quantizada, o modelo continua tendo solução exata e realmente a introdução de flutuações quânticas corrige o problema da entropia.

Outro fator que chamou a atenção para os modelos quantizados decorre do intenso estudo de transições de fase quânticas [25], isto é, transições a temperatura zero. Em particular, como mostrado em [26], a versão quântica do modelo esférico exibe além do comportamento clássico não trivial uma transição de fase quântica caracterizada por novos expoentes críticos. Mais do que isso, a versão quântica é equivalente ao modelo sigma não-linear no limite do número de campos tendendo para o infinito. Essa conexão desperta bastante interesse, visando a possibilidade de aplicação de métodos da teoria de campos. Um dos nossos objetivos é explorar essa conexão por meio de uma abordagem da teoria de campos, a partir da qual se torna mais natural a extensão do modelo para o caso supersimétrico.

Originalmente introduzida no contexto da teoria de campos como uma generalização da simetria de Poincaré, a supersimetria tem sido frequentemente aplicada ao estudo de problemas em outras áreas. Na situação não-relativística, de principal interesse para a física da matéria condensada, a supersimetria foi introduzida por Nicolai em [27, 28], no estudo de sistemas de spins, e por Witten em [29], na construção da mecânica quântica supersimétrica. Exemplos de aplicações em diversos sistemas da matéria condensada podem ser encontrados em [30, 31, 32].

Quanto à estrutura, a tese está dividida basicamente em duas partes. A primeira parte é mais voltada para a teoria de campos, englobando os capítulos 1 a 4 . A segunda parte, envolvendo os capítulos 5 a 7, é direcionada para a mecânica estatística.

O primeiro capítulo é dedicado a algumas motivações do trabalho além de uma discussão geral sobre teorias com anisotropia entre as coordenadas espaciais e temporal, indicando a construção de modelos escalares, espinoriais, de calibre e gravitacionais. No segundo capítulo, discutimos aspectos do procedimento de renormalização em teorias escalares anisotrópicas, culminando com um estudo do grupo de renormalização. Esse estudo é prosseguido no capítulo 3, num modelo com uma interação de Yukawa, que além do campo escalar envolve um campo espinorial. O capítulo 4 trata do estudo de simetrias ao nível quântico, com um estudo das identidades de Ward, generalizando o método dos produtos normais para o caso de teorias anisotrópicas. Também discutimos aplicações desse método. 
A segunda parte, iniciada no capítulo 5, apresenta alguns aspectos básicos envolvidos na solução do modelo de Ising, como a dualidade entre altas e baixas temperaturas e a conexão com a teoria de campos, e também na solução do modelo esférico clássico que constitui o ponto de partida para os estudos dos capítulos seguintes. Realmente, no capítulo 6, consideramos a versão quântica do modelo esférico, numa abordagem via integração funcional e discutimos a conexão com o modelo sigma não-linear. No capítulo 7, apresentamos a extensão supersimétrica do modelo esférico quântico e finalizamos com a análise de seu comportamento crítico. Um sumário do trabalho, destacando pontos importantes e algumas conclusões, é apresentado nas Considerações Finais. 


\section{Capítulo 1}

\section{Aspectos Gerais}

O objetivo deste capítulo introdutório é expor de uma maneira geral diversos aspectos e possibilidades envolvidas no estudo de teorias com anisotropia, bem como as motivações as quais nos levam a considerar tais teorias. Em particular, discutimos a conexão com as transições de fase contínuas (segunda ordem) que ocorrem em muitos sistemas da matéria condensada e que nos leva ao estudo de modelos estatísticos.

\subsection{Teorias Relativísticas com Derivadas de Ordem Superior}

Uma maneira muito natural de obter teorias relativísticas com um melhor comportamento ultravioleta consiste em incluir na parte livre da lagrangiana termos com derivadas superiores. Por exemplo, no caso de um campo escalar real podemos escrever

$$
\mathcal{L}=-\frac{1}{2} \varphi\left(\alpha \square^{2}+\square+m^{2}\right) \varphi+\mathcal{L}_{\text {int }}(\varphi) .
$$

Duas questões imediatamente surgem: 1. Qual o significado físico desse novo tipo de termo? Ou, o que estamos incluindo de novo na teoria? 2. Isso pode nos trazer algum problema? Em princípio, não há uma razão física evidente para justificar a presença de um termo assim. As respostas a essas perguntas estão relacionadas. Para entender melhor isso é conveniente reescrever a lagrangiana acima como

$$
\mathcal{L}=-\frac{1}{2} \alpha \varphi\left(\square+m_{+}^{2}\right)\left(\square+m_{-}^{2}\right) \varphi+\mathcal{L}_{\text {int }}(\varphi),
$$

em que definimos

$$
m_{ \pm}^{2} \equiv \frac{1}{2 \alpha}\left(1 \pm \sqrt{1-4 m^{2} \alpha}\right),
$$


com $0<4 m^{2} \alpha \leq 1$. Introduzindo campos $\varphi_{1}$ e $\varphi_{2}$ por meio de

$$
\varphi_{1,2} \equiv \frac{\sqrt{\alpha}}{\sqrt{m_{+}^{2}-m_{-}^{2}}}\left(\square+m_{ \pm}^{2}\right) \varphi
$$

obtemos

$$
\mathcal{L}=-\frac{1}{2} \varphi_{1}\left(\square+m_{-}^{2}\right) \varphi_{1}+\frac{1}{2} \varphi_{2}\left(\square+m_{+}^{2}\right) \varphi_{2}+\mathcal{L}_{\text {int }}\left(\varphi_{1}, \varphi_{2}\right) .
$$

Esse resultado mostra explicitamente que o propagador livre do campo $\varphi_{2}$ tem um sinal errado. De uma forma geral, isso está associado à presença de estados com norma negativa na teoria, denominados fantasmas. Se não houver interação, esses estados simplesmente se desacoplam não trazendo problemas. Entretanto, a interação acopla esses graus com os graus físicos e isso pode trazer consequências drásticas. De fato, esses graus de liberdade são incompatíveis com a unitariedade, que é uma propriedade indispensável para a interpretação física da teoria.

Observe que a lagrangiana (1.5) tem uma estrutura análoga a de uma teoria com uma regularização tipo Pauli-Villars. Teorias formuladas dessa maneira tornam-se, em geral, renormalizáveis. Um exemplo importante disso é o caso da gravitação. Como mostrado por Stelle em [33], ao incluir termos com derivadas superiores na ação de Einstein-Hilbert,

$$
S=\int d^{D} x \sqrt{-g}\left[\gamma \frac{1}{\kappa^{2}} R+\frac{1}{\kappa^{4}} \Lambda-\frac{1}{\alpha^{2}}\left(R_{\mu \nu} R^{\mu \nu}-\frac{1}{3} R^{2}\right)+\beta R^{2}\right]
$$

a teoria torna-se renormalizável. Outra questão pertinente é se essa teoria recupera a relatividade geral de Einstein? A relatividade geral de Einstein pode ser pensada como uma aproximação de longas distâncias da ação (1.6) , isto é, como uma ação efetiva de baixas energias

$$
S_{e f} \sim \int d^{D} x \sqrt{-g}\left(\gamma \frac{1}{\kappa^{2}} R+\frac{1}{\kappa^{4}} \Lambda\right) .
$$

Um último comentário antes de concluir esta seção é que o surgimento de estados tipo fantasma está diretamente relacionado com a inclusão de derivadas temporais de ordem superior. Essa observação traz novas possibilidades para tratar o problema. Abrindo mão da simetria de Lorentz, podemos ter um efeito semelhante ao anterior, no sentido da melhora do comportamento ultravioleta, adicionando-se termos de derivadas de ordem superior envolvendo apenas a parte espacial. Antes de considerar concretamente essa questão, vamos discutir um pouco as relações entre uma anisotropia suave entre espaço e tempo e a simetria de Lorentz. 


\subsection{Quebra Suave da Simetria de Lorentz}

No estudo sobre a possibilidade de restauração da simetria de Lorentz em algum limite de baixa energia, basicamente devemos estudar o fluxo dos parâmetros da teoria mediante o grupo de renormalização. Nesse sentido é importante entender algumas características desse fluxo no caso de teorias com uma quebra suave da simetria de Lorentz.

Para uma teoria envolvendo apenas um tipo de campo, o modelo mais simples que podemos escrever é dado pela lagrangiana

$$
\mathcal{L}=\frac{1}{2} \partial_{0} \varphi \partial_{0} \varphi-\frac{b_{\varphi}^{2}}{2} \partial_{i} \varphi \partial_{i} \varphi-\frac{m^{2}}{2} \varphi^{2}-\frac{\lambda}{3 !} \varphi^{3}
$$

Esse modelo é renormalizável em $5+1$ dimensões espaço-temporais. Estamos supondo que um termo linear no campo foi adicionado à lagrangiana com o coeficiente ajustado de modo a eliminar todos os diagramas com uma linha externa (tadpoles). Para obter o comportamento efetivo do parâmetro $b_{\varphi}$, o qual é definido por $\beta_{b_{\varphi}^{2}} \equiv \mu \partial b_{\varphi}^{2} / \partial \mu$, sendo $\mu$ a escala introduzida no processo de renormalização, basta analisar a função de dois pontos. Usando a equação do grupo de renormalização de t'Hooft-Weinberg, obtemos o resultado $\beta_{b_{\varphi}^{2}}=0$. Isso nada mais é que o reflexo da simetria de Lorentz: a velocidade da luz não é um parâmetro efetivo no sentido do grupo de renormalização. Isso justifica a escolha do sistema natural de unidades, isto é, temos liberdade para escolher $c=1$.

A análise de grupo de renormalização acima é na realidade completamente desnecessária. De fato, a lagrangiana (1.8) corresponde a escolha de um sistema de unidades em que $c=b_{\varphi}$. Obviamente isso não prejudica a simetria de Lorentz. Além disso, pensando em termos da ação, esse fator pode sempre ser eliminado por meio de um reescalonamento da coordenada espacial $x^{i}, x^{i} \rightarrow b_{\varphi} x^{i}$. O resultado é um fator $b_{\varphi}^{5}$ global na ação, que é fisicamente irrelevante.

No caso de teorias envolvendo mais que um campo, temos a possibilidade de considerar fatores diferentes acompanhando as derivadas espaciais. Para exemplificar isso, consideremos o modelo de Yukawa em $3+1$ dimensões:

$$
\begin{aligned}
\mathcal{L} & =\frac{1}{2} \partial_{0} \varphi \partial_{0} \varphi-\frac{b_{\varphi}^{2}}{2} \partial_{i} \varphi \partial_{i} \varphi-\frac{m^{2}}{2} \varphi^{2}+\bar{\psi}\left(i \gamma^{0} \partial_{0}+i b_{\psi} \gamma^{i} \partial_{i}-M\right) \psi \\
& +i g \bar{\psi} \gamma^{5} \psi \varphi-\frac{\lambda}{4 !} \varphi^{4}
\end{aligned}
$$

Note que, ao contrário da situação anterior, agora não é possível eliminar simultaneamente os parâmetros $b_{\varphi}$ e $b_{\psi}$ por meio de reescalonamento da coordenada espacial. Temos então, uma quebra da simetria de Lorentz! Nessa situação, o comportamento desses parâmetros não é trivial. Podemos esperar, contudo, que quando $b_{\varphi}=b_{\psi}$, as funções $\beta_{b_{\varphi}^{2}}$ e $\beta_{b_{\psi}^{2}}$ se anulem, pois esse é o caso da simetria de Lorentz. Mas, e no caso $b_{\varphi} \neq b_{\psi}$ ? Qual é o comportamento das soluções? Para obter o comportamento dos parâmetros $b_{\varphi}$ e 
$b_{\psi}$, devemos analisar as funções de dois pontos tanto do campo escalar como do campo espinorial. Dessa forma, repetindo o procedimento descrito acima, encontramos

$$
\beta_{b_{\varphi}^{2}}=\frac{1}{4 \pi^{2}} \frac{\left(b_{\varphi}+b_{\psi}\right)}{b_{\psi}^{3}}\left(b_{\varphi}-b_{\psi}\right) g^{2}
$$

e

$$
\beta_{b_{\psi}}=-\frac{1}{6 \pi^{2}} \frac{1}{b_{\varphi}\left(b_{\varphi}+b_{\psi}\right)^{2}}\left(b_{\varphi}-b_{\psi}\right) g^{2} .
$$

Como esperado, quando $b_{\varphi}=b_{\psi}$, temos $\beta_{b_{\varphi}^{2}}=\beta_{b_{\psi}}=0$. Agora, vamos analisar a situação em que $b_{\varphi} \neq b_{\psi}$. Temos duas possibilidades:

1. $b_{\varphi}>b_{\psi}$. Nesse caso, a função $b_{\varphi}$ é monotonamente crescente, enquanto que $b_{\psi}$ é monotonamente decrescente. Esse comportamento é ilustrado, de maneira qualitativa, na figura 1.1. Como $b_{\varphi}>b_{\psi}$, estamos a direita do ponto em que os fluxos se cruzam. Assim,

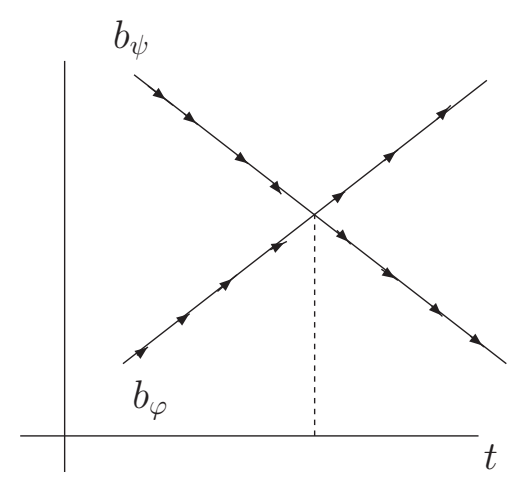

Figura 1.1: Fluxo dos Parâmetros para $b_{\varphi}>b_{\psi}$ conforme $t$ aumenta.

conforme $t\left(t=\ln \mu / \mu_{0}\right)$ vai aumentando, estamos cada vez mais distantes de recuperar a simetria de Lorentz. Por outro lado, ao diminuir $t$, o fluxo é revertido e, então, os dois parâmetros tendem para o ponto em que se tornam iguais.

2. $b_{\varphi}<b_{\psi}$. Agora, a situação se inverte, ou seja, a função $b_{\varphi}$ torna-se monotonamente decrescente, enquanto que $b_{\psi}$ torna-se monotonamente crescente. Ilustramos esse comportamento na figura 1.2. Novamente, nos encontramos à direita do ponto onde os fluxos se encontram. De fato, independentemente da condição inicial, sempre estamos localizados à direita do ponto em que $b_{\varphi}=b_{\psi}$; portanto, quando $t$ vai diminuindo, o fluxo sempre nos leva a esse ponto. Uma vez atingido esse ponto, de acordo com as equações (1.10) e (1.11), as funções $\beta_{b_{\varphi}^{2}}$ e $\beta_{b_{\psi}^{2}}$ tornam-se iguais a zero e não mudam mais. Então, na verdade, o fluxo à esquerda do ponto deve ser uma linha horizontal, como mostrado na figura 1.3, e não como os diagramas acima. Isso nos leva à seguinte conclusão. A simetria de Lorentz é um ponto fixo do grupo de renormalização, conforme diminuímos a escala de energia. Em outras palavras, a simetria de Lorentz é uma consequência inevitável do fluxo do grupo de renormalização no limite de baixas energias. 


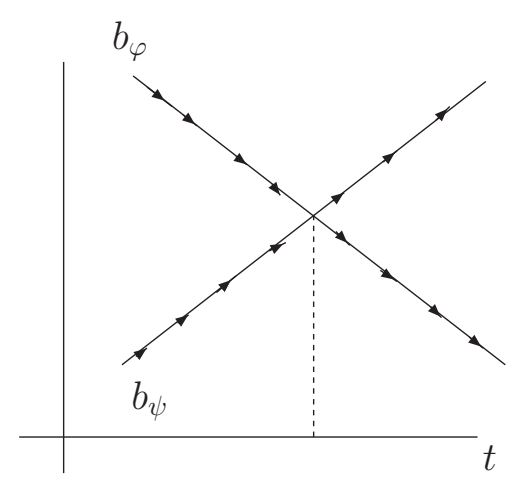

Figura 1.2: Fluxo dos Parâmetros para $b_{\varphi}<b_{\psi}$ conforme $t$ aumenta.

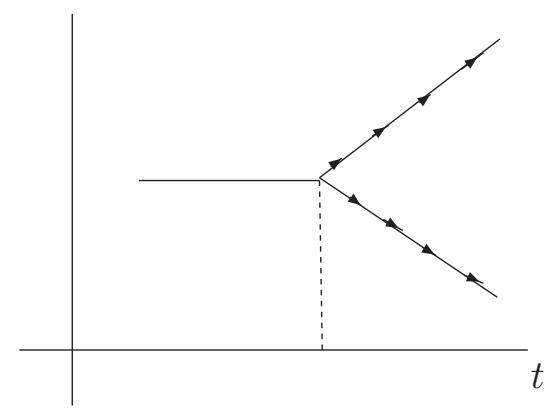

Figura 1.3: Fluxo dos Parâmetros conforme $t$ aumenta.

A modificação feita na lagrangiana (1.9), introduzindo os parâmetros $b_{\varphi}$ e $b_{\psi}$, corresponde a uma quebra suave da simetria de Lorentz por meio de uma anisotropia entre o espaço e o tempo. A teoria não sofre nenhuma mudança estrutural. A contagem de potências é a mesma e consequentemente o grau de divergência superficial bem como a dimensão crítica permanecem inalterados. Em suma, a estrutura UV não é modificada.

Entretanto, podemos introduzir a anisotropia de uma maneira mais drástica, isto é, adicionando-se à lagrangiana termos envolvendo derivadas espacias de ordem superior, por exemplo, $\varphi \Delta^{2} \varphi$, em que $\Delta$ é o laplaciano. Então, será que algum mecanismo de restauração da simetria de Lorentz semelhante ao mostrado acima sobrevive a esse tipo de quebra? Um dos principais objetivos da primeira parte deste trabalho é discutir essa questão. Queremos estudar a estrutura UV de algumas teorias e quais as consequências dessas modificações no fluxo do grupo de renormalização dos parâmetros envolvidos. Trabalhos nessa direção também foram apresentados em [34, 35]. 


\subsection{Transições de Fase Contínuas}

Conforme mencionamos na Introdução, vamos discutir um pouco sobre a anisotropia entre o espaço e o tempo no contexto das transições de fases [14, 15, 16].

Quando um sistema passa por uma transição de fase, ele muda de propriedades físicas, ou seja, ele muda de ordem. Para caracterizar essa mudança, introduzimos uma grandeza, chamada parâmetro de ordem, possuindo as seguintes propriedades: em uma das fases é nulo (fase desordenada) e na outra fase é não nulo (fase ordenada). Estamos nos restringindo a transições contínuas (ou de segunda ordem). Assim, nas proximidades da região crítica, o parâmetro de ordem é pequeno, justificando o desenvolvimento da energia livre como uma série de potências desse parâmetro de ordem, conhecida como expansão de Landau-Ginzburg [36, 37].

Citamos aqui alguns exemplos típicos de transições de fase em sistemas físicos e o parâmetro de ordem correspondente. Numa transição para-ferromagnética, o parâmetro de ordem é a magnetização. Para uma transição gás-líquido, o parâmetro de ordem adequado é a diferença entre os volumes específicos das fases coexistentes, $v_{G}-v_{L}$. Em tais sistemas, encontrar um parâmetro de ordem adequado é fácil e até mesmo natural. Entretanto, isso nem sempre é uma tarefa simples.

A expansão de Landau-Ginzburg mencionada anteriormente, fornece uma descrição geral do comportamento crítico, isto é, ela não descreve um sistema específico. Mas, as transições ocorrem em tantos sistemas diferentes, cada um com as suas particularidades e suas características microscópicas. Qual a razão para considerar uma teoria geral de transições de fase? A resposta a essa pergunta nos leva ao conceito de universalidade, que é a característica que faz o estudo dos fenômenos críticos tão especial e fascinante.

Imediatamente nas vizinhanças da região crítica, certas grandezas termodinâmicas, como o calor específico e a suscetibilidade, apresentam um comportamento peculiar, com divergências que são governadas por um conjunto de expoentes críticos. Nesse sentido, os expoentes críticos descrevem a natureza das singularidades e caracterizam a transição de fase. Todas as transições que possuem o mesmo conjunto de expoentes, pertencem à mesma classe de universalidade. O fato mais marcante é que as classes de universalidade são determinadas por poucos fatores, essencialmente a dimensão espacial do sistema considerado e as simetrias envolvidas.

O que está por trás da universalidade é o fato que, na região crítica, o comprimento de correlação é a única escala de comprimento da teoria. Distante do ponto crítico, o comprimento de correlação é da ordem do espaçamento entre os sítios da rede, no caso de um sólido. Assim, as propriedades microscópicas são importantes e conduzem a características muito distintas entre diferentes materiais. Porém, nas proximidades do ponto crítico, o comprimento de correlação diverge (torna-se da ordem do tamanho do sistema) e as características microscópicas do sistema passam a ser irrelevantes. 
Nas proximidades do ponto crítico, o comprimento de correlação $\xi$ comporta-se como

$$
\xi \sim|t|^{-\nu}
$$

em que $\nu$ é o expoente crítico do comprimento de correlação e $t$ é algum parâmetro que mede a distância ao ponto crítico. Por exemplo, numa transição que ocorre quando o sistema passa por uma temperatura crítica $T_{c}$, ele é usualmente escolhido como $t \equiv$ $\left(T-T_{c}\right) / T_{c}$. Em adição às correlações espaciais, o sistema exibe correlações temporais. A escala de tempo típica que as flutuações levam para decair é definida pelo tempo de correlação $\tau_{c}$, caracterizado pelo seguinte comportamento:

$$
\tau_{c} \sim|t|^{-z \nu} \sim \xi^{z}
$$

sendo $z$ o expoente crítico dinâmico. Esse expoente determina a anisotropia entre o espaço e o tempo, no sentido descrito acima.

O expoente crítico dinâmico é inerente ao estudo de transições de fase em sistemas quânticos. No caso de um sistema clássico, a função de partição se fatora,

$$
Z=Z_{\text {Cinética }} Z_{\text {Potencial }}
$$

Em outras palavras, a estática e a dinâmica se desacoplam. Assim, podemos estudar o comportamente crítico com parâmetros de ordem independentes do tempo, mas com uma dependência espacial. Para uma hamiltoniana quântica, o desacoplamento acima não ocorre e nos obriga a considerar modelos com parâmetros de ordem dependentes do tempo também.

É nesse contexto que passamos a estudar o ponto de Lifshitz. Um ponto de Lifshitz em um diagrama de fases é definido como o ponto de encontro entre uma fase desordenada, uma fase uniforme e uma fase modulada. Esse tipo de estrutura ocorre em diversos sistemas magnéticos, cristais líquidos, polímeros entre outros. Na discussão que segue usaremos a linguagem de sistemas magnéticos, baseada em modelos tipo Ising.

Diagramas exibindo essa estrutura de fases decorrem de sistemas envolvendo interações competitivas, favorecendo diferentes ordenamentos. Como um protótipo, consideraremos um modelo tipo ANNNI generalizado (do inglês, axial-next-nearest-neighbor Ising) sobre uma rede quadrada bidimensional com espaçamento $a$, com interações ferromagnéticas entre primeiros vizinhos, anti-ferromagnéticas entre segundos vizinhos e uma interação indefinida a priori entre vizinhos diagonais. Além disso, por uma questão de conveniência, vamos supor que as variáveis de spin em cada sítio sejam contínuaș e que haja competição em todas as direções. A hamiltoniana é dada por

$$
\mathcal{H}=-J_{1} \sum_{<\mathbf{r}, \mathbf{r}^{\prime}>} S_{\mathbf{r}} S_{\mathbf{r}^{\prime}}-J_{2} \sum_{\ll \mathbf{r}, \mathbf{r}^{\prime} \gg} S_{\mathbf{r}} S_{\mathbf{r}^{\prime}}-J_{3} \sum_{\prec \mathbf{r}, \mathbf{r}^{\prime} \succ} S_{\mathbf{r}} S_{\mathbf{r}^{\prime}}
$$

\footnotetext{
${ }^{1}$ Variáveis tipo as do modelo esférico. Discutiremos em detalhes esse modelo na parte final deste trabalho.
} 
com $J_{1}>0$ favorecendo o ordenamento ferromagnético, $J_{2}<0$ o anti-ferromagnético e $J_{3}$ sem sinal definido a priori. Os símbolos $<>$ e $\ll \gg$ indicam soma sobre primeiros e segundos vizinhos, respectivamente, enquanto $\prec \succ$ significa soma entre vizinhos nas diagonais. A ilustração geométrica dessa situação é mostrada na figura 1.4. O caso ANNNI usual corresponde a $J_{3}=0$.

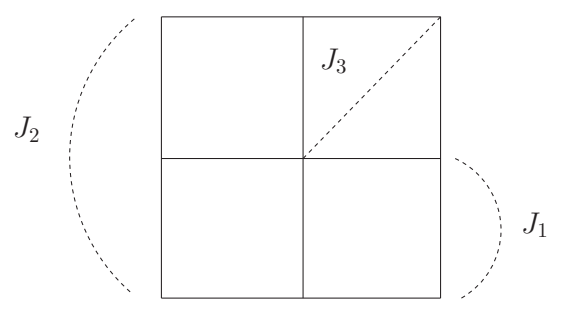

Figura 1.4: Interações competitivas na rede quadrada.

Para quantizar o modelo, precisamos atribuir uma dinâmica, o que pode ser feito por meio da adição de um termo cinético. A lagrangiana correspondente pode ser escrita como

$$
\mathcal{L}=\frac{1}{2} \sum_{\mathbf{r}}\left(\frac{d S_{\mathbf{r}}}{d t}\right)^{2}+J_{1} \sum_{<\mathbf{r}, \mathbf{r}^{\prime}>} S_{\mathbf{r}} S_{\mathbf{r}^{\prime}}+J_{2} \sum_{\ll \mathbf{r}, \mathbf{r}^{\prime} \gg} S_{\mathbf{r}} S_{\mathbf{r}^{\prime}}+J_{3} \sum_{\prec \mathbf{r}, \mathbf{r}^{\prime} \succ} S_{\mathbf{r}} S_{\mathbf{r}^{\prime}}
$$

A partir dessa expressão podemos construir a teoria quântica por meio da integração funcional, por exemplo. Esse procedimento de quantização de modelos estatísticos será discutido em detalhe nos últimos capítulos, no estudo de transições de fase quânticas.

Para entender qualitativamente a conexão entre (1.16) e os modelos de teoria de campos estudados em grande parte deste trabalho, e além disso o efeito das interações entre vizinhos no modelo correspondente, vamos estudar o limite contínuo da rede $a \rightarrow 0$. Lembre que, nas proximidades do ponto crítico o comprimento de correlação diverge $\xi \gg a$. Em primeiro lugar é conveniente escrever a lagrangiana acima explicitando as interações da seguinte forma

$$
\begin{aligned}
\mathcal{L} & =\frac{1}{2} \sum_{x, y}\left(\frac{d S_{x, y}}{d t}\right)^{2}+J_{1} \sum_{x, y}\left(S_{x+a, y} S_{x, y}+S_{x, y+a} S_{x, y}\right)+J_{2} \sum_{x, y}\left(S_{x+2 a, y} S_{x, y}+S_{x, y+2 a} S_{x, y}\right) \\
& +J_{3} \sum_{x, y}\left(S_{x+a, y+a} S_{x, y}+S_{x+a, y} S_{x, y+a}\right)
\end{aligned}
$$

Observe que no limite do contínuo a interação entre primeiros vizinhos $S_{x+a, y} S_{x, y}$ pode ser identificada com uma derivada

$$
\left(\frac{\partial S_{x, y}}{\partial x}\right)^{2} \sim\left(\frac{S_{x+a, y}-S_{x, y}}{a}\right)^{2}
$$


Assim, o termo cruzado correspondente à interação entre primeiros vizinhos é escrito como

$$
S_{x+a, y} S_{x, y} \sim S_{x, y}^{2}-\frac{1}{2} a^{2}\left(\frac{\partial S_{x, y}}{\partial x}\right)^{2}
$$

levando em conta a somatória sobre $x$ e $y$. Nas deduções seguintes isso também está subentendido. A expressão para $S_{x, y+a} S_{x, y}$ é semelhante. A interação entre segundos vizinhos $S_{x+2 a, y} S_{x, y}$ pode ser identificada a partir da derivada segunda

$$
\left(\frac{\partial^{2} S_{x, y}}{\partial x^{2}}\right)^{2} \sim\left(\frac{S_{x+2 a, y}-2 S_{x+a, y}+S_{x, y}}{a^{2}}\right)^{2}
$$

de modo que

$$
S_{x+2 a, y} S_{x, y} \sim S_{x, y}^{2}-2 a^{2}\left(\frac{\partial S_{x, y}}{\partial x}\right)^{2}+\frac{1}{2} a^{4}\left(\frac{\partial^{2} S_{x, y}}{\partial x^{2}}\right)^{2}
$$

com uma expressão análoga para $S_{x, y+2 a} S_{x, y}$. Por fim, as interações diagonais são escritas a partir de termos contendo derivadas mistas

$$
S_{x+a, y+a} S_{x, y}+S_{x+a, y} S_{x, y+a} \sim-6 S_{x, y}^{2}+a^{2}\left(\frac{\partial S_{x, y}}{\partial x}\right)^{2}+a^{2}\left(\frac{\partial S_{x, y}}{\partial y}\right)^{2}+\frac{1}{2} a^{4}\left(\frac{\partial^{2} S_{x, y}}{\partial x \partial y}\right)^{2} .
$$

Reunindo todas as contribuições, a lagrangiana (1.17) torna-se

$$
\begin{aligned}
\mathcal{L} & =\int d^{2} r\left\{\frac{1}{2}\left(\frac{\partial S}{\partial t}\right)^{2}+2\left(J_{1}+J_{2}-3 J_{3}\right) S^{2}-\frac{1}{2} a^{2}\left(J_{1}+4 J_{2}-2 J_{3}\right)(\nabla S)^{2}\right. \\
& \left.+\frac{1}{2} a^{4} J_{2}\left[\left(\frac{\partial^{2} S}{\partial x^{2}}\right)^{2}+\left(\frac{\partial^{2} S}{\partial y^{2}}\right)^{2}+\frac{J_{3}}{J_{2}}\left(\frac{\partial^{2} S}{\partial x \partial y}\right)^{2}\right]\right\},
\end{aligned}
$$

em que estamos omitindo a dependência espaço-temporal da variável $S(x, y, t)$. O ponto de Lifshitz é caracterizado pelo anulamento do termo $(\nabla S)^{2}$, o que ocorre quando $J_{1}+$ $4 J_{2}-2 J_{3}=0$ (No caso usual com $J_{3}=0$ o ponto de Lifishitz se reduz a $J_{1}+4 J_{2}=0$ [38]). Na teoria quantizada dizemos que esse é um ponto de Lifshitz quântico! A sua característica especial é que o sistema exibe um escalonamento anisotrópico entre espaço e tempo, com um expoente crítico dinâmico $z=2$. Por outro lado, quando $J_{2}=0 \mathrm{e}$ $J_{3}=0$, a lagrangiana exibe a simetria relativística, correspondendo a $z=1$. Da discussão acima fica claro que a inclusão de interações entre vizinhos mais distantes é equivalente a considerar termos com derivadas espaciais de ordens mais altas, podendo dar origem a valores arbitrários de $z$.

Para as propostas da teoria de campos, buscamos uma lagrangiana com invariância rotacional (espacial), o que requer que a última linha de (1.23) seja reconhecida como 
$\left(\nabla^{2} S\right)^{2}$. Isso é obtido com a escolha $J_{3}=2 J_{2}$ e fazendo integrações por partes no termo com as derivadas mistas,

$$
\mathcal{L}=\int d^{2} r\left[\frac{1}{2}\left(\frac{\partial S}{\partial t}\right)^{2}+2\left(J_{1}-5 J_{2}\right) S^{2}-\frac{1}{2} a^{2} J_{1}(\nabla S)^{2}+\frac{1}{2} a^{4} J_{2}\left(\nabla^{2} S\right)^{2}\right] .
$$

A lagrangiana (1.24) tem exatamente a forma de uma teoria escalar livre com a inclusão de um termo envolvendo derivadas espaciais de ordem superior. Nesse sentido, podemos tentar generalizar essa estrutura para teorias com campos de diferentes carateres como espinores, campos de calibre e gravitacionais.

\subsection{Construção de Modelos}

\subsubsection{Campos Escalares e Espinorias}

Não há uma maneira unívoca para a construção de teorias exibindo anisotropia entre espaço e tempo. Por exemplo, para um campo escalar, a modificação da parte livre mais geral envolve todas as potências do laplaciano entre 1 e $z$ :

$$
\mathcal{L}_{0}=\frac{1}{2} \partial_{0} \varphi \partial_{0} \varphi+\frac{1}{2} \varphi\left(\alpha_{1}^{2} \Delta-\alpha_{2}^{2} \Delta^{2}+\cdots+\alpha_{z}^{2} \Delta^{z}\right) \varphi-\frac{m^{2}}{2} \varphi^{2} .
$$

No estudo sobre a possibilidade de restauração da simetria de Lorentz é necessário considerar os termos de ordem inferior a $z$, principalmente o termo usual $(z=1)$, pois essencialmente, devemos comparar a relevância desses operadores em diferentes regimes de energia. Mais do que isso, eles podem ser necessários para garantir a renormalizabilidade da teoria. Por outro lado, a presença dos termos de ordem inferior traz dificuldades técnicas praticamente intransponíveis na análise de diagramas envolvendo mais que um laço.

Além dos tipos de termos a serem considerados, temos ainda uma ambiguidade na questão dos sinais que acompanham cada termo. Esses sinais serão escolhidos de modo que a relação de dispersão seja uma expressão positiva definida,

$$
E^{2}=\alpha_{1}^{2} \mathbf{p}^{2}+\alpha_{2}^{2}\left(\mathbf{p}^{2}\right)^{2}+\cdots+\alpha_{z}^{2}\left(\mathbf{p}^{2}\right)^{z}+m^{2} .
$$

Para teorias envolvendo campos fermiônicos a construção é semelhante e requer apenas um cuidado especial para garantir que a lagrangiana seja hermitiana,

$$
\mathcal{L}_{0}=\bar{\psi} i \gamma^{0} \partial_{0} \psi+\bar{\psi}\left[\alpha_{1} i \gamma^{i} \partial_{i}+\alpha_{2}\left(i \gamma^{i} \partial_{i}\right)^{2}+\cdots+\alpha_{z}\left(i \gamma^{i} \partial_{i}\right)^{z}-M\right] \psi
$$

Existe ainda outra possibilidade de construir operadores com altas derivadas espaciais, a saber,

$$
\gamma^{i}(-\Delta)^{\frac{z-1}{2}} \partial_{i}
$$


Observe que termos com essa estrutura trazem dificuldades quando $z$ é par, conduzindo a um operador não-local na parte cinética. Por esse motivo, descartaremos termos desse tipo em nossos estudos, os quais serão restritos ao caso $z=2$. Quando $z$ é um número ímpar, (1.28) se reduz ao tipo de termos presentes em (1.27).

Podemos tentar um raciocínio análogo sobre a relação de dispersão para decidir os sinais. Por simplicidade, vamos nos concentrar diretamente no caso $z=2$. Sendo assim, a posição do pólo do propagador associado à lagrangiana (1.27) fornece

$$
E^{2}=\alpha_{1} \mathbf{p}^{2}+\left(\alpha_{2} \mathbf{p}^{2}+M\right)^{2}
$$

Essa análise não é suficiente para fixar os sinais. Qualquer que seja o sinal do termo com derivada superior $\alpha_{2}$, a relação de dispersão será positiva definida. Nesse caso não temos uma escolha preferencial e, então, podemos considerar o sinal positivo, tal como escrito acima.

Esse tipo de construção não afeta a estrutura de interação da teoria (a estrutura topológica dos diagramas). Ela modifica apenas as partes livres, que são relevantes para a contagem de potências e, consequentemente, para o grau de divergência superficial. Os propagadores têm um melhor comportamento ultravioleta e de uma forma geral há uma melhoria nas propriedades de convergência das funções de Green, favorecendo a renormalização. Entretanto, no caso de teorias com simetria de calibre esse efeito não é tão evidente, pois a introdução da anisotropia dá origem a novos termos de interação. Reservamos a seção seguinte para discutir um pouco melhor essa questão.

Para concluir, mencionamos que quando temos uma teoria envolvendo diferentes campos (sejam de mesmo caráter ou não) interagindo é desejável que o grau de anisotropia seja o mesmo. Em outras palavras, os propagadores são modificados com derivadas da mesma ordem para todos os campos, correspondendo ao mesmo grau z. Essa homogeneidade conduz a uma contagem de potências anisotrópica muito natural, em que é possível discutir de maneira geral as propriedades de renormalização. Isso também será estudado em detalhe.

\subsubsection{Teorias de Calibre}

Em constraste com a discussão precedente, a construção de teorias de calibre com anisotropia acaba afetando a parte de interação. Isso decorre do requisito da simetria de calibre no acoplamento com campos de matéria. Sabemos que um produto arbitrário de derivadas covariantes tem a lei de transformação desejada, isto é,

$$
\left(D_{\mu} D_{\nu} \cdots D_{\rho} \Phi\right)^{\prime}=e^{i q \Lambda(x)} D_{\mu} D_{\nu} \cdots D_{\rho} \Phi
$$

em que $\Phi$ designa genericamente um campo (escalar ou espinorial) cuja transformação é da forma $\Phi^{\prime}=e^{i q \Lambda(x)} \Phi$, e a derivada covariante é definida da maneira usual, $D_{\mu} \equiv$ 
$\partial_{\mu}-i q A_{\mu}$. Assim, para introduzir a anisotropia sem prejudicar a simetria de calibre, os termos adicionais envolverão derivadas covariantes de ordem superior e isso introduz novos termos de interação, podendo modificar drasticamente as propriedades da teoria.

Para ilustrar esse fato, consideremos a eletrodinâmica com $z=2$ [35],

$$
\mathcal{L}=\frac{1}{2} F_{0 i} F_{0 i}-\frac{\sigma_{1}^{2}}{4} F_{i j} F_{i j}-\frac{\sigma_{2}^{2}}{4} F_{i j} \Delta F_{i j}+\bar{\psi} i \gamma^{0} D_{0} \psi+\bar{\psi}\left(\alpha_{1} i \gamma^{i} D_{i}+\alpha_{2}\left(i \gamma^{i} D_{i}\right)^{2}-M\right) \psi
$$

De passagem, observe que o grau de anisotropia é uniforme entre o campos espinorial e de calibre, conforme mencionado anteriormente. Além da interação usual,

$$
\mathcal{L}_{i n t}=q \bar{\psi} \gamma^{0} \psi A_{0}-\alpha_{1} q \bar{\psi} \gamma^{i} \psi A^{i}
$$

teremos novos tipos de termos, tais como

$$
\mathcal{L}_{\text {int }}=-\alpha_{2} q^{2} \bar{\psi} \psi A^{i} A^{i}-i \alpha_{2} q \bar{\psi} \gamma^{i} \gamma^{j} \psi \partial_{i} A^{j}+\cdots
$$

representados graficamente na figura 1.5. Assim, no cálculo da função de três pontos
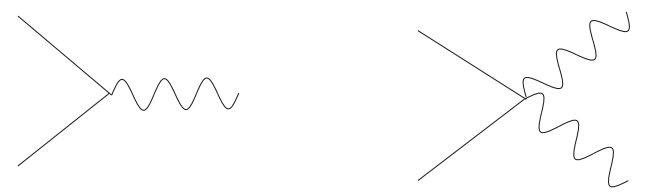

Figura 1.5: Vértices de Interação.

$\left\langle\bar{\psi} \psi A_{\mu}\right\rangle$, por exemplo, teremos dois diagramas da mesma ordem contribuindo, mostrados na figura 1.6. Além do diagrama usual, o primeiro diagrama da figura 1.6, temos a

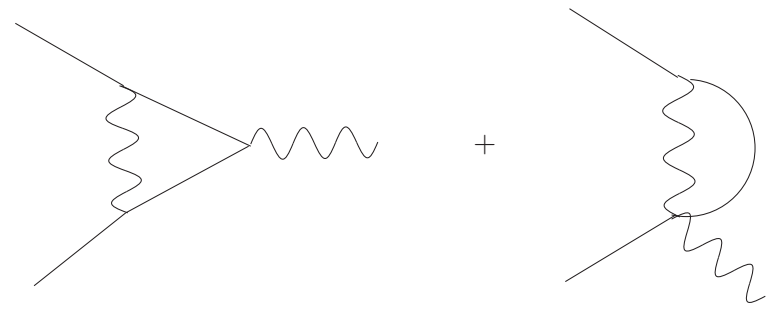

Figura 1.6: Diagramas que contribuem para a função $\beta_{q}$.

contribuição adicional do segundo diagrama. Uma vez que esses diagramas contribuem para a função $\beta_{q}$, não é claro a priori se isso afetará ou não a estabilidade da teoria no ultravioleta e no infravermelho. Teorias não-abelianas também já foram consideradas na literatura [39], mas não as discutiremos aqui. 


\subsubsection{Gravitação}

Um dos casos de maior interesse para aplicar as idéias descritas anteriormente tratase da gravitação [13]. Em particular, há uma forma especialmente adequada para a implementação da anisotropia. De fato, o formalismo ADM [40, 41, que essencialmente refere-se à decomposição $3+1$ do espaço-tempo em espaço e tempo, é muito conveniente.

A ação ADM é construída a partir da foliação do espaço-tempo $M$ em superfícies de Cauchy, $\Sigma_{t}$, parametrizadas por uma função global (tempo) $t$, ou seja, cada superfície $\Sigma_{t}$ corresponde a um $t$ fixo. A métrica do espaço-tempo, $g_{a b}$, induz uma métrica espacial $h_{a b}$ (métrica tri-dimensional Riemanniana) sobre cada $\Sigma_{t}$ de acordo com

$$
h_{a b}=g_{a b}+n_{a} n_{b},
$$

em que $n^{a}$ é um vetor unitário tipo tempd2 $\left(n^{a} n_{a}=-1\right)$ normal à superfície $\Sigma_{t}$. Note que $n^{a} h_{a b}=0$, tal que $h_{a b}$ pode ser interpretado como uma projeção sobre $\Sigma_{t}$.

Seja $t^{a}$ um campo vetorial sobre $M$, satisfazendo a condição $t^{a} \nabla_{a} t=1$. $\nabla_{a}$ é o operador derivativo compatível com $g_{a b}, \nabla_{a} g_{b c}=0$. Podemos decompor $t^{a}$ nas partes normal e tangencial, definindo assim a função lapso, $N$, e o vetor shift, $N^{a}$, em relação a $t^{a}:$

$$
N \equiv-t^{a} n_{a}
$$

$\mathrm{e}$

$$
N_{a} \equiv h_{a b} t^{b}
$$

A figura [1.7 ilustra essa situação. Outro objeto fundamental nessa construção é a curva-

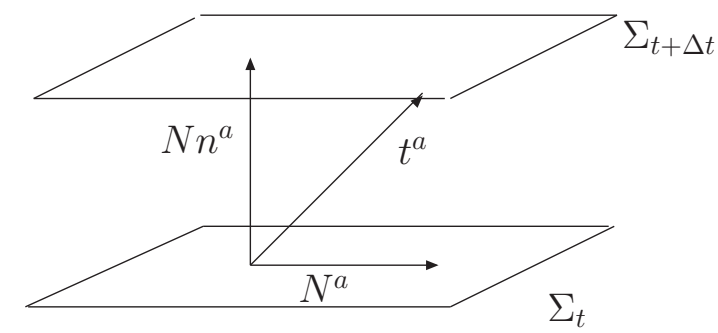

Figura 1.7: Definição da função lapso, $N$, e do vetor shift $N^{a}$.

tura extrínseca $K_{a b}$, definida por

$$
K_{a b} \equiv h_{a}{ }^{c} \nabla_{c} n_{b}=h_{a}{ }^{c} h_{b}{ }^{d} \nabla_{c} n_{d}=\frac{1}{2} £_{n} h_{a b},
$$

em que $£_{n}$ é a derivada de Lie e está relacionada com a derivada temporal, de modo que

$$
K_{a b}=\frac{1}{2 N}\left(\dot{h}-D_{a} N_{b}-D_{b} N_{a}\right)
$$

\footnotetext{
${ }^{2}$ Nesta seção estamos usando a métrica com assinatura $(-1,1,1,1)$, usual na relatividade geral.
} 
$D_{a}$ é o operador derivativo compatível com $h_{a b}, D_{a} h_{b c}=0$. A partir desses ingredientes é possível relacionar as quantidades do espaço-tempo (quadridimensionais) com as quantidades tridimensionais sobre $\Sigma_{t}$. Em particular, o escalar de Ricci pode ser escrito como

$$
R={ }^{(3)} R-K^{2}+K_{a b} K^{a b},
$$

em que $K \equiv K_{a}{ }^{a}$. A observação importante é que há uma separação entre as derivadas temporais e espaciais. De fato, as derivadas temporais estão presentes apenas na curvatura extrínseca $K_{a b}$, ao passo que o escalar de curvatura ${ }^{(3)} R$ envolve apenas derivadas espaciais. Outra expressão útil na construção da ação é a relação entre os determinantes das métricas $\sqrt{-g}=N \sqrt{h}$. Dessa forma, a ação de Einstein-Hilbert, escrita de acordo com a decomposição $3+1$, torna-se

$$
S=\int d^{3} x d t N \sqrt{h}\left[{ }^{(3)} R-K^{2}+K_{a b} K^{a b}\right] .
$$

Conforme mencionamos, essa forma é especialmente adequada para a implementação da anisotropia entre o espaço e tempo. A anisotropia pode ser introduzida de uma maneira muito natural simplesmente adicionando-se termos com potências maiores de ${ }^{(3)} R,{ }^{(3)} R_{a b}$ $\mathrm{e}^{(3)} R_{a b c d}$ :

$$
S=\int d^{3} x d t N \sqrt{h}\left[K_{a b} K^{a b}-\sigma K^{2}+\alpha_{1}{ }^{(3)} R+\alpha_{2}{ }^{(3)} R^{2}+\alpha_{3}{ }^{(3)} R_{a b}{ }^{(3)} R^{a b}+\cdots\right],
$$

de acordo com o grau de anisotropia que desejamos considerar [13.

Após essa discussão da implementação da anisotropia em modelos com diversos tipos de campos, passaremos a estudar as propriedades de renormalização de forma um pouco mais aprofundada, culminando com uma análise do grupo de renormalização em modelos específicos. Esse programa será iniciado no capítulo seguinte, a partir do estudo de campos escalares e continuará no terceiro capítulo com um estudo do modelo de Yukawa. 


\section{Capítulo 2}

\section{Grupo de Renormalização - Teorias Escalares}

O objetivo deste capítulo é apresentar alguns aspectos técnicos inerentes ao procedimento de renormalização em teorias com anisotropia, por meio do estudo de modelos envolvendo apenas campos escalares. Tais aspectos são necessários para determinar o comportamento dos parâmetros efetivos da teoria, o que é obtido via grupo de renormalização [42].

\section{$2.1 \quad$ Teoria Livre}

Em teorias com derivadas espaciais de ordem superior, as dimensões das coordenadas temporal e espacial são adequadamente definidas como

$$
\operatorname{Dim}\left[x^{0}\right] \equiv z \text { e } \operatorname{Dim}\left[x^{i}\right] \equiv 1 .
$$

Esse tipo de anisotropia conduz a uma contagem de potências muito natural, que permite determinar o grau de divergência superficial e, portanto, discutir de maneira sistemática a renormalizabilidade da teoria.

Retomando a discussão inicial do primeiro capítulo, a parte livre da lagrangiana de uma teoria escalar possui a estrutura

$$
\mathcal{L}_{0}=\frac{1}{2} \partial_{0} \varphi \partial_{0} \varphi+\frac{1}{2} \varphi\left(\alpha_{1}^{2} \Delta-\alpha_{2}^{2} \Delta^{2}+\cdots+\alpha_{z}^{2} \Delta^{z}\right) \varphi-\frac{m^{2}}{2} \varphi^{2},
$$

em que $\Delta \equiv \nabla^{2}$ é o laplaciano em $d$ dimensões espaciais. O propagador correspondente é

$$
\Delta(k)=\frac{i}{k_{0}^{2}-\alpha_{1}^{2} \mathbf{k}^{2}+\cdots+(-1)^{z} \alpha_{z}^{2}\left(\mathbf{k}^{2}\right)^{z}-m^{2}+i \epsilon} .
$$


Os termos relevantes para a contagem de potências da teoria são: o da derivada temporal, que conduz ao fator $k_{0}^{2}$ e o da derivada espacial de ordem mais alta, conduzindo ao fator $\left(\mathbf{k}^{2}\right)^{z}$. De acordo com (2.1), esses dois fatores escalam com a potência $2 z$.

De uma maneira um pouco superficial e imprecisa, a idéia da restauração da simetria de Lorentz está relacionada ao fato que propagador acima exibe dois comportamentos distintos, conforme a faixa de valores do momento (por simplicidade, vamos assumir que apenas $\alpha_{1}$ e $\alpha_{z}$ são diferentes de zero). Primeiro, para valores pequenos do momento,

$$
\frac{i}{k_{0}^{2}-\alpha_{1}^{2} \mathbf{k}^{2}-\alpha_{z}^{2}\left(\mathbf{k}^{2}\right)^{z}-m^{2}+i \epsilon} \sim \frac{i}{k_{0}^{2}-\alpha_{1}^{2} \mathbf{k}^{2}-m^{2}+i \epsilon}, \quad \mathbf{k}^{2} \rightarrow 0
$$

Nesse limite, o propagador tem um comportamento relativístico. Segundo, para valores grandes do momento,

$$
\frac{i}{k_{0}^{2}-\alpha_{1}^{2} \mathbf{k}^{2}-\alpha_{z}^{2}\left(\mathbf{k}^{2}\right)^{z}-m^{2}+i \epsilon} \sim \frac{i}{k_{0}^{2}-\alpha_{z}^{2}\left(\mathbf{k}^{2}\right)^{z}-m^{2}+i \epsilon}, \quad \mathbf{k}^{2} \rightarrow \infty
$$

e o comportameto ultravioleta é melhorado.

Uma questão que surge é o que acontece numa teoria em interação, em que a estrutura perturbativa envolve integrais correndo sobre todos os valores dos momentos. A simetria de Lorentz poderia emergir num limite de baixas energias? A resposta a essa pergunta é delicada e envolve diversos aspectos que serão discutidos ao longo deste capítulo.

Isso pode fornecer um mecanismo interessante para transformar teorias relativísticas não-renormalizáveis em teorias renormalizáveis sem a simetria de Lorentz, que pode emergir num limite de baixas energias. Para investigar esse mecanismo de um modo mais preciso é imprescindível realizar um estudo sobre o grupo de renormalização. Essencialmente, devemos analisar os parâmetros efetivos da teoria e verificar se há um processo natural nesse sentido.

\subsection{Renormalização}

A primeira etapa da renormalização trata-se da contagem de potências. De fato, para ter controle sobre o comportamento ultravioleta, precisamos determinar o grau de divergência superficial da teoria. Essa é uma tarefa simples. O que deve ser notado é que a dimensão do espaço de fase é efetivamente $d+z$ e cada propagador escala com uma potência $2 z$ do momento no denominador. Então, dado um diagrama arbitrário $G$, com $L$ loops e $n$ linhas internas, temos

$$
d(G)=L(d+z)-n(2 z)
$$

numa teoria sem acoplamento derivativo. Usando a relação de Euler, $L=n-V+1$, sendo $V$ o número de vértices, e a relação topológica, $2 n+N=\sum \nu_{a}$, em que $N$ é o 
número de linhas externas e $\nu_{a}$ é o número de linhas unindo-se no vértice $a$, obtemos

$$
d(G)=(d+z)-\left(\frac{d-z}{2}\right) N-\sum_{a}\left[(d+z)-\left(\frac{d-z}{2}\right) \nu_{a}\right] .
$$

Observe que as dimensões do campo escalar e do vértice são respectivamente

$$
\operatorname{Dim}[\varphi]=\frac{d-z}{2}
$$

$\mathrm{e}$

$$
\operatorname{Dim}\left[V_{a}\right]=\left(\frac{d-z}{2}\right) \nu_{a}
$$

Assim, podemos classificar os vértices em: super-renormalizáveis, se $\operatorname{Dim}\left[V_{a}\right]<d+z$; renormalizáveis, se $\operatorname{Dim}\left[V_{a}\right]=d+z$; e não-renormalizáveis, se $\operatorname{Dim}\left[V_{a}\right]>d+z$. É claro que a contagem de potências acima pode ser generalizada sem dificuldades para incluir acoplamentos derivativos e campos fermiônicos. Faremos isso a medida que for necessário, no capítulo seguinte. Para uma interação do tipo

$$
\mathcal{L}_{\text {int }}=-\frac{\lambda}{P !} \varphi^{P}
$$

com $P>2$, a dimensão da constante de acoplamento será

$$
\operatorname{Dim}[\lambda]=\frac{z(P+2)-d(P-2)}{2} .
$$

Teorias renormalizáveis requerem uma constante de acoplamento $\lambda$ adimensional, ou seja, $\operatorname{Dim}[\lambda]=0\left(\operatorname{Dim}\left[V_{a}\right]=d+z\right)$.

Agora, seguem alguns comentários sobre o esquema de renormalização empregado. Dado um diagrama $G$ primitivamente divergente, ou seja, sem subdiagramas divergentes, ele pode ser feito finito se o seu integrando $I(G)$ for substituído por

$$
R(G)=\left(1-t^{d(G)}\right) I_{G}=I_{G}-\sum_{s=0}^{\left[\frac{d(G)}{z}\right]} \frac{p_{0}^{s}}{s !} \frac{\partial^{s}}{\partial p_{0}^{s}} \sum_{n=0}^{d(G)-s z} \frac{p_{i_{1}} \ldots p_{i_{n}}}{n !} \frac{\partial}{\partial p_{i_{1}}} \ldots \frac{\partial}{\partial p_{i_{n}}} I_{G}
$$

em que $[x]$ é o maior inteiro menor ou igual a $x$. O momento $p_{0}$ designa simbolicamente um conjunto de momentos externos, dependendo do número de linhas externas do diagrama em questão. Todas as derivadas são calculadas com momento externo igual a zero. Nos cálculos de um laço envolvidos neste trabalho, aplicaremos o resultado acima apenas para evidenciar os pólos dos diagramas regularizados dimensionalmente. Uma observação relevante é que essa é uma regularização dimensional modificada, envolvendo apenas o número de dimensões espaciais $d$. Nossa prescrição de renormalização, consiste em remover as partes de pólo, o qual é usualmente chamado esquema de subtração minimal MS. Em geral, as funções beta dependem das constantes de acoplamento bem como do ponto de renormalização $\mu$, por meio da razão $\mu / m$. Entretanto, no esquema de subtração minimal de pólos, elas não dependem de $\mu$ e, por questões dimensionais, não podem depender da massa $m$. 


\subsection{O modelo $\varphi^{3}$ e Liberdade Assintótica}

O modelo $\varphi^{3}$ é especial por diversos motivos. Ele é o modelo mais simples exibindo propriedades não trivias características do procedimento de renormalização. A estrutura topólogica dos diagramas em ordens mais baixas restringe-se a diagramas de um laço. Para análise do grupo de renormalização é sufuciente determinar as partes divergentes (partes de pólos). Como ficará claro mais adiante, devido as potências diferentes das componentes do momento no propagador na situação anisotrópica, o cálculo da parte divergente dos diagramas torna-se uma tarefa complicada para gráficos de um laço e traz dificuldades praticamente intransponíveis para gráficos com mais de um laço, exceto em situações muito especiais.

No caso usual isotrópico, o modelo $\varphi^{3}$ é renormalizável em seis dimensões espaçotemporais. Além disso, ele compartilha com as teorias de calibre não-abelianas a propriedade ímpar de liberdade assintótica no ultravioleta. Realmente, a função beta correspondente é dada por [43]

$$
\beta_{\lambda}=-\frac{3}{4(4 \pi)^{3}} \lambda^{3}
$$

mostrando que a constante de acoplamento efetiva decresce com o aumento da energia. Assim, na situação anisotrópica, será possível investigar se essa propriedade é de fato inalterada, e qual o efeito disso sobre os parâmetros efetivos da teoria.

A lagrangiana, para o caso $z=2$, é dada por

$$
\mathcal{L}=\frac{1}{2} \partial_{0} \varphi \partial_{0} \varphi-\frac{b^{2}}{2} \partial_{i} \varphi \partial_{i} \varphi-\frac{\alpha^{2}}{2} \varphi \Delta^{2} \varphi-\frac{m^{2}}{2} \varphi^{2}-\frac{\lambda}{3 !} \varphi^{3} .
$$

A teoria torna-se renormalizável em $d=10$ dimensões espaciais, com o grau de divergência

$$
d(G)=12-4 N .
$$

Então, as funções de vértice divergentes são aquelas com: $N=1,2,3$ linhas externas. Para $N=1(d(G)=8)$, supomos que há um termo linear em $\varphi$ adicionado à lagrangiana cujo coeficiente é ajustado de modo a cancelar todos os tadpoles. As demais funções têm a divergência superficial $d(G)=4$ para $N=2$ e $d(G)=0$ para $N=3$. Os diagramas correspondentes, até a ordem de um laço, são mostrados nas figuras 2.1 e 2.2.

\subsection{Parte Divergente das Funções de Vértice}

No estudo do grupo de renormalização precisamos extrair as partes divergentes das funções de vértice. Discutiremos agora um procedimento para este fim. A forma geral 


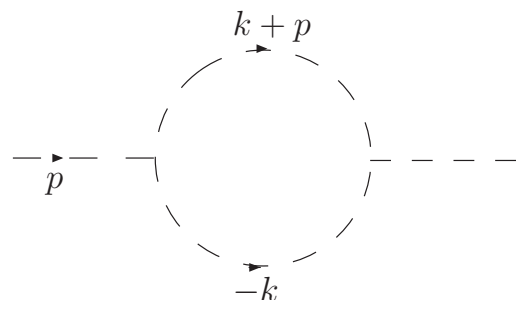

Figura 2.1: Função de dois pontos.

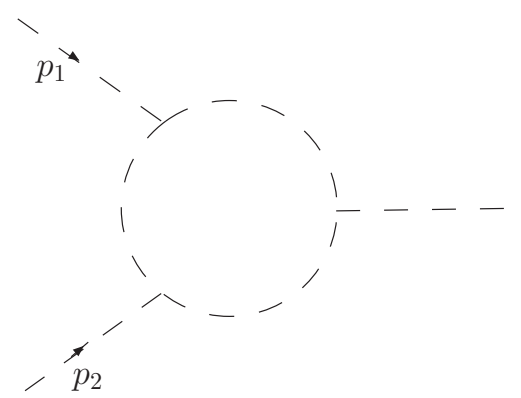

Figura 2.2: Função de três pontos.

das integrais envolvidas é

$$
I(x, y) \equiv \int \frac{d k_{0}}{2 \pi} \frac{d^{d} k}{(2 \pi)^{d}} \frac{|\mathbf{k}|^{x}}{\left[k_{0}^{2}-b^{2} \mathbf{k}^{2}-m^{2}-\alpha^{2}\left(\mathbf{k}^{2}\right)^{2}+i \epsilon\right]^{y}},
$$

em que $d, x$ e $y$ são tais que a integral é no máximo quarticamente divergente. Usando a parametrização de Schwinger generalizada,

$$
\frac{i^{y}}{(A+i \epsilon)^{y}}=\frac{1}{\Gamma(y)} \int_{0}^{\infty} d \gamma \gamma^{y-1} e^{i \gamma(A+i \epsilon)},
$$

podemos escrever $I(x, y)$ como

$$
I(x, y) \equiv \frac{1}{i^{y} \Gamma(y)} \int_{0}^{\infty} d \gamma \gamma^{y-1} e^{i \gamma\left(-m^{2}+i \epsilon\right)} \int \frac{d k_{0}}{2 \pi} e^{i \gamma k_{0}^{2}} \int \frac{d^{d} k}{(2 \pi)^{d}}|\mathbf{k}|^{x} e^{i \gamma\left[-b^{2} \mathbf{k}^{2}-\alpha^{2}\left(\mathbf{k}^{2}\right)^{2}\right]} .
$$

A integral em $k_{0}$ é trivial,

$$
\int \frac{d k_{0}}{2 \pi} e^{i \gamma k_{0}^{2}}=\frac{1}{2 \pi}\left(\frac{\pi}{-i \gamma}\right)^{\frac{1}{2}}
$$

enquanto que a integral na parte espacial necessita de um pouco mais de trabalho. Empregando coordenadas esféricas,

$$
I I \equiv \frac{\Omega_{d}}{(2 \pi)^{d}} \int_{0}^{\infty} d|\mathbf{k}||\mathbf{k}|^{x+d-1} e^{i \gamma\left[-b^{2} \mathbf{k}^{2}-\alpha^{2}\left(\mathbf{k}^{2}\right)^{2}\right]},
$$


com o ângulo sólido em $d$ dimensões $\Omega_{d} \equiv 2 \pi^{\frac{d}{2}} / \Gamma(d / 2)$, o resultado pode ser escrito em termos da função hipergeométrica confluente ${ }_{1} F_{1}$

$$
\begin{aligned}
I I & =\frac{\Omega_{d}}{(2 \pi)^{d}} \frac{1}{4 \alpha^{2}}\left(i \alpha^{2} \gamma\right)^{-\frac{(d+x)}{4}}\left[\alpha^{2} \Gamma\left(\frac{d+x}{4}\right){ }_{1} F_{1}\left(\frac{d+x}{4}, \frac{1}{2}, \frac{i b^{4} \gamma}{4 \alpha^{2}}\right)\right. \\
& \left.-b^{2}\left(i \alpha^{2} \gamma\right)^{\frac{1}{2}} \Gamma\left(\frac{d+x+2}{4}\right){ }_{1} F_{1}\left(\frac{d+x+2}{4}, \frac{3}{2}, \frac{i b^{4} \gamma}{4 \alpha^{2}}\right)\right] .
\end{aligned}
$$

A parte divergente da integração em $\gamma$ está localizada no limite inferior $(\gamma \rightarrow 0)$, tal que podemos considerar apenas os termos dominantes em ${ }_{1} F_{1}$ :

$$
{ }_{1} F_{1}\left(\frac{d+x}{4}, \frac{1}{2}, \frac{i b^{4} \gamma}{4 \alpha^{2}}\right)=1+\frac{i b^{4}(d+x) \gamma}{8 \alpha^{2}}+\mathcal{O}\left(\gamma^{2}\right)
$$

e

$$
{ }_{1} F_{1}\left(\frac{d+x+2}{4}, \frac{3}{2}, \frac{i b^{4} \gamma}{4 \alpha^{2}}\right)=1+\mathcal{O}(\gamma) .
$$

Note que, na última expansão, podemos considerar apenas o primeiro termo, por causa do fator $\gamma^{\frac{1}{2}}$ multiplicando-a em (2.21). Todas as potências de $\gamma$ maiores que as consideradas produzem termos finitos. Dessa forma, substituindo (2.19) e (2.21) em (2.18) levando em conta as expansões acima, ficamos com

$$
\begin{aligned}
I(x, y) & =\frac{\pi^{\frac{1}{2}}}{8 \pi} \frac{\Omega_{d}}{(2 \pi)^{d}} \frac{\alpha^{-\frac{(d+x+4)}{2}}}{i^{y} \Gamma(y)}\left[\alpha^{2} \Gamma\left(\frac{d+x}{4}\right)\right. \\
& \times \int_{0}^{\infty} d \gamma\left(1+\frac{i b^{4}(d+x) \gamma}{8 \alpha^{2}}\right) \gamma^{y-1}\left(\frac{1}{-i \gamma}\right)^{\frac{1}{2}}(i \gamma)^{-\frac{(d+x)}{4}} e^{i \gamma\left(-m^{2}+i \epsilon\right)} \\
& \left.-b^{2} \alpha \Gamma\left(\frac{d+x+2}{4}\right) \int_{0}^{\infty} d \gamma \gamma^{y-1}\left(\frac{1}{-i \gamma}\right)^{\frac{1}{2}}(i \gamma)^{-\frac{(d+x-2)}{4}} e^{i \gamma\left(-m^{2}+i \epsilon\right)}\right] \\
& +\operatorname{termos} \text { finitos } \\
& =\frac{\pi^{\frac{1}{2}}}{8 \pi} \frac{\Omega_{d}}{(2 \pi)^{d}} \frac{\alpha^{-\frac{(d+x+4)}{2}}}{i^{y} \Gamma(y)}\left\{\alpha^{2} \Gamma\left(\frac{d+x}{4}\right)\right. \\
& \times\left[(-1)^{\frac{2-d-x}{8}}\left(i m^{2}\right)^{\frac{2+d+x-4 y}{4}} \Gamma\left(\frac{-2-d-x+4 y}{4}\right)\right. \\
& \left.+\frac{i b^{4}(d+x)}{8 \alpha^{2}}(-1)^{\frac{2-d-x}{8}}\left(i m^{2}\right)^{\frac{-2+d+x-4 y}{4}} \Gamma\left(\frac{2-d-x+4 y}{4}\right)\right] \\
& \left.-i b^{2} \alpha \Gamma\left(\frac{d+x+2}{4}\right)(-1)^{\frac{-(d+x)}{8}}\left(i m^{2}\right)^{\frac{d+x-4 y}{4}} \Gamma\left(\frac{-d-x+4 y}{4}\right)\right\} \\
& +\operatorname{termos} \text { finitos } \\
& \equiv f(d) g(x, y)+\text { termos finitos, }
\end{aligned}
$$


em que definimos uma parte multiplicativa independente de $x$ e $y$,

$$
f(d) \equiv \frac{\pi^{\frac{1}{2}}}{8 \pi} \frac{\Omega_{d}}{(2 \pi)^{d}}
$$

e outra dependente como $g(x, y)$ contendo os demais fatores. Com o resultado acima, estamos prontos para determinar as partes divergentes das funções de vértice relevantes.

\subsection{Função de 2 pontos}

Iniciaremos nosso estudo considerando a função de vértice de dois pontos até a ordem de um laço. A expressão completa do diagrama 2.1 é

$$
\frac{\lambda^{2}}{2} \int \frac{d k_{0}}{2 \pi} \frac{d^{d} k}{(2 \pi)^{d}} \frac{1}{k^{2}-\alpha^{2}\left(\mathbf{k}^{2}\right)^{2}-m^{2}+i \epsilon} \frac{1}{(k+p)^{2}-\alpha^{2}\left[(\mathbf{k}+\mathbf{p})^{2}\right]^{2}-m^{2}+i \epsilon},
$$

em que $k^{2} \equiv k_{0}^{2}-b^{2} \mathbf{k}^{2}$. Seja $I(p, k)$ o integrando dessa expressão. De acordo com (2.12), as partes divergentes podem ser encontradas a partir da aplicação do operador de Taylor $t^{4}$ sobre $I(p, k)$ :

$$
\begin{aligned}
t^{4} I(p, k) & =I(0, k)+\left.\frac{p_{i} p_{j}}{2 !} \frac{\partial^{2} I(p, k)}{\partial p_{i} \partial p_{j}}\right|_{p=0}+\left.\frac{p_{0} p_{0}}{2 !} \frac{\partial^{2} I(p, k)}{\partial p_{0} \partial p_{0}}\right|_{p=0} \\
& +\left.\frac{p_{i} p_{j} p_{k} p_{l}}{4 !} \frac{\partial^{4} I(p, k)}{\partial p_{i} \partial p_{j} \partial p_{k} \partial p_{l}}\right|_{p=0}
\end{aligned}
$$

Observe que as derivadas atuam apenas sobre o segundo propagador, dependente do momento externo. Devido à extensão, vamos calcular cada um dos termos acima separadamente. É conveniente introduzir alguns tensores completamente simétricos que serão úteis em nossos cálculos:

$$
\begin{gathered}
T_{i j k l}^{(0)} \equiv \delta_{i j} \delta_{k l}+\delta_{i k} \delta_{j l}+\delta_{j k} \delta_{i l}, \\
T_{i j k l}^{(2)}(k) \equiv \delta_{i j} k_{l} k_{k}+\delta_{i k} k_{l} k_{j}+\delta_{j l} k_{k} k_{i}+\delta_{j k} k_{l} k_{i}+\delta_{i l} k_{k} k_{j}+\delta_{l k} k_{i} k_{j}
\end{gathered}
$$

$\mathrm{e}$

$$
T_{i j k l}^{(4)}(k) \equiv k_{i} k_{j} k_{k} k_{l} \text {. }
$$

Os índices superiores indicam a potência do momento. 


\section{Derivadas em relação a $p_{i} p_{j} p_{k} p_{l}$}

Vamos iniciar com o termo de quatro derivadas em (2.27). É conveniente definir o coeficiente

$$
\left.D_{i j k l} \equiv \frac{\partial^{4} I(p)}{\partial p_{i} \partial p_{j} \partial p_{k} \partial p_{l}}\right|_{p=0} .
$$

A aplicação de todas derivadas fornece

$$
\begin{aligned}
D_{i j k l} & =\left(\frac{2 h^{2}}{[\operatorname{den}]^{4}}+\frac{8 \alpha^{2}}{[\operatorname{den}]^{3}}\right) T_{i j k l}^{(0)}+\left(\frac{6 h^{3}}{[\operatorname{den}]^{5}}+\frac{48 \alpha^{2} h}{[\operatorname{den}]^{4}}\right) T_{i j k l}^{(2)}(k) \\
& +\left(\frac{24 h^{4}}{[\operatorname{den}]^{6}}+\frac{288 \alpha^{2} h^{2}}{[\operatorname{den}]^{5}}+\frac{384 \alpha^{4}}{[\operatorname{den}]^{4}}\right) T_{i j k l}^{(4)}(k),
\end{aligned}
$$

em que den $\equiv k^{2}-\alpha^{2}\left(\mathbf{k}^{2}\right)^{2}-m^{2}+i \epsilon$ and $h \equiv 2\left(b^{2}+2 \alpha^{2} \mathbf{k}^{2}\right)$. Agora, considerando apenas os termos divergentes, segue que,

$$
\begin{aligned}
D_{i j k l} & =\left(\frac{32 \alpha^{4}\left(\mathbf{k}^{2}\right)^{2}}{[\operatorname{den}]^{4}}+\frac{8 \alpha^{2}}{[\operatorname{den}]^{3}}\right) T_{i j k l}^{(0)}+\left(\frac{384 \alpha^{6}\left(\mathbf{k}^{2}\right)^{3}}{[\operatorname{den}]^{5}}+\frac{192 \alpha^{4} \mathbf{k}^{2}}{[\operatorname{den}]^{4}}\right) T_{i j k l}^{(2)}(k) \\
& +\left(\frac{6144 \alpha^{8}\left(\mathbf{k}^{2}\right)^{4}}{[\operatorname{den}]^{6}}+\frac{4608 \alpha^{6}\left(\mathbf{k}^{2}\right)^{2}}{[\operatorname{den}]^{5}}+\frac{384 \alpha^{4}}{[\operatorname{den}]^{4}}\right) T_{i j k l}^{(4)}(k)+\text { termos finitos. }
\end{aligned}
$$

Tomando vantagem da simetria rotacional, podemos fazer as seguintes mudanças

$$
T_{i j k l}^{(2)}(k) \rightarrow \frac{2 \mathbf{k}^{2}}{d} T_{i j k l}^{(0)}
$$

$\mathrm{e}$

$$
T_{i j k l}^{(4)}(k) \rightarrow \frac{\left(\mathbf{k}^{2}\right)^{2}}{d(d+2)} T_{i j k l}^{(0)}
$$

Com isso, (2.33) torna-se

$$
\begin{aligned}
D_{i j k l} & =\left(\frac{32 \alpha^{4}\left(\mathbf{k}^{2}\right)^{2}}{[\operatorname{den}]^{4}}+\frac{8 \alpha^{2}}{[\operatorname{den}]^{3}}+\frac{768 \alpha^{6}\left(\mathbf{k}^{2}\right)^{4}}{d[\operatorname{den}]^{5}}+\frac{384 \alpha^{4}\left(\mathbf{k}^{2}\right)^{2}}{d[\text { den }]^{4}}+\frac{6144 \alpha^{8}\left(\mathbf{k}^{2}\right)^{6}}{d(d+2)[\text { den }]^{6}}\right. \\
& \left.+\frac{4608 \alpha^{6}\left(\mathbf{k}^{2}\right)^{4}}{d(d+2)[\operatorname{den}]^{5}}+\frac{384 \alpha^{4}\left(\mathbf{k}^{2}\right)^{2}}{d(d+2)[\operatorname{den}]^{4}}\right) T_{i j k l}^{(0)}+\text { termos finitos. }
\end{aligned}
$$

Para obter o resultado final, devemos calcular a integral sobre os momentos,

$$
\frac{\lambda^{2}}{2} \frac{p_{i} p_{j} p_{k} p_{l}}{4 !} \int \frac{d k_{0}}{2 \pi} \frac{d^{d} k}{(2 \pi)^{d}} D_{i j k l} .
$$


Usando o resultado (2.24), obtemos

$$
\begin{aligned}
& \frac{\lambda^{2}}{2} \frac{p_{i} p_{j} p_{k} p_{l}}{4 !} f(d)\left(32 \alpha^{4} g(4,4)+8 \alpha^{2} g(0,3)+\frac{768 \alpha^{6} g(8,5)}{d}\right. \\
+ & \frac{384 \alpha^{4} g(4,4)}{d}+\frac{6144 \alpha^{8} g(12,6)}{d(d+2)}+\frac{4608 \alpha^{6} g(8,5)}{d(d+2)} \\
+ & \left.\frac{384 \alpha^{4} g(4,4)}{d(d+2)}\right) T_{i j k l}^{(0)}(k)+\text { termos finitos. }
\end{aligned}
$$

O cálculo do resíduo em $d=10$, fornece

$$
-i \frac{11}{3932160 \pi^{5}} \frac{\lambda^{2}}{\alpha^{3}} \frac{1}{d-10}\left(\mathbf{p}^{2}\right)^{2} .
$$

\section{Derivadas em relação a $p_{0} p_{0}$}

O termo de duas derivadas resulta em

$$
\left.\frac{\partial^{2} I(p)}{\partial p_{0} \partial p_{0}}\right|_{p=0}=\frac{-2}{[\operatorname{den}]^{3}}+\frac{8 k_{0}^{2}}{[\operatorname{den}]^{4}},
$$

que pode ser escrito como

$$
\left.\frac{\partial^{2} I(p)}{\partial p_{0} \partial p_{0}}\right|_{p=0}=\frac{6}{[\operatorname{den}]^{3}}+\frac{8 \alpha^{2}\left(\mathbf{k}^{2}\right)^{2}}{[\operatorname{den}]^{4}}+\text { termos finitos . }
$$

Agora, podemos usar (2.24). A expressão completa será

$$
\frac{\lambda^{2}}{4} p_{0}^{2} f(d)\left[6 g(0,3)+8 \alpha^{2} g(4,4)\right]+\text { termos finitos. }
$$

Daí, obtemos a parte divergente

$$
-i \frac{1}{393216 \pi^{5}} \frac{\lambda^{2}}{\alpha^{5}} \frac{1}{d-10} p_{0}^{2}
$$

\section{Derivadas em relação a $p_{i} p_{j}$}

Aplicando as duas derivadas, segue que

$$
\begin{aligned}
\left.\frac{\partial^{2} I(p)}{\partial p_{i} \partial p_{j}}\right|_{p=0} & =\left(\frac{32 b \alpha^{2}\left(\mathbf{k}^{2}\right)^{2}}{d[\text { den }]^{4}}+\frac{32 \alpha^{4}\left(\mathbf{k}^{2}\right)^{3}}{d[\text { den }]^{4}}+\frac{2 b^{2}}{[\text { den }]^{3}}+\frac{4 \alpha^{2} \mathbf{k}^{2}}{[\text { den }]^{3}}+\frac{8 \alpha^{2} \mathbf{k}^{2}}{d[\text { den }]^{3}}\right) \delta_{i j} \\
& + \text { termos finitos. }
\end{aligned}
$$


A expressão completa é

$$
\frac{\lambda^{2}}{4} \mathbf{p}^{2} f(d)\left[\frac{32 b^{2} \alpha^{2}}{d} g(4,4)+\frac{32 \alpha^{4}}{d} g(6,4)+2 b^{2} g(0,3)+4 \alpha^{2} g(2,3)+\frac{8 \alpha^{2}}{d} g(2,3)\right]
$$

+ termos finitos.

A parte divergente obtida é

$$
-i \frac{5}{393216 \pi^{5}} \frac{b^{2} \lambda^{2}}{\alpha^{5}} \frac{1}{d-10} \mathbf{p}^{2}
$$

\section{Termo sem derivadas}

Esse é o último termo do operador de Taylor (2.27) que necessitamos avaliar. A expressão é

$$
\frac{\lambda^{2}}{2} \int \frac{d k_{0}}{2 \pi} \frac{d^{d} k}{(2 \pi)^{d}} \frac{1}{\left[k^{2}-m^{2}-\alpha^{2}\left(\mathbf{k}^{2}\right)^{2}+i \epsilon\right]^{2}} .
$$

De acordo com (2.24), temos

$$
\frac{\lambda^{2}}{2} f(d) g(0,2)
$$

com a parte divergente

$$
i \frac{1}{262144 \pi^{5}} \frac{\lambda^{2}}{\alpha^{7}} \frac{1}{d-10}\left(-5 b^{4}+4 \alpha^{2} m^{2}\right) .
$$

\subsection{Função de 3 pontos}

Passemos a considerar a função de vértice de 3 pontos até um laço, mostrada na figura 2.2. O grau de divergência superficial é $d(G)=0$, ou seja, logaritmicamente divergente. A expressão correspondente ao diagrama é

$$
\begin{aligned}
& \lambda^{3} \int \frac{d k_{0}}{2 \pi} \frac{d^{d} k}{(2 \pi)^{d}} \frac{1}{k^{2}-\alpha^{2}\left(\mathbf{k}^{2}\right)^{2}-m^{2}+i \epsilon} \frac{1}{\left(k+p_{1}\right)^{2}-\alpha^{2}\left[\left(\mathbf{k}+\mathbf{p}_{1}\right)^{2}\right]^{2}-m^{2}+i \epsilon} \\
\times \quad & \frac{1}{\left(p_{2}-k\right)^{2}-\alpha^{2}\left[\left(\mathbf{p}_{2}-\mathbf{k}\right)^{2}\right]^{2}-m^{2}+i \epsilon} .
\end{aligned}
$$

Seja $J\left(p_{1}, p_{2}, k\right)$ o integrando acima. O operador de Taylor é simplesmente

$$
t^{0} J\left(p_{1}, p_{2}, k\right)=J(0,0, k)
$$


Então, precisamos avaliar apenas $J(0,0, k)$ :

$$
\lambda^{3} \int \frac{d k_{0}}{2 \pi} \frac{d^{d} k}{(2 \pi)^{d}} \frac{1}{\left[k^{2}-\alpha^{2}\left(\mathbf{k}^{2}\right)^{2}-m^{2}+i \epsilon\right]^{3}} .
$$

De acordo com (2.24), temos

$$
\lambda^{3} f(d) g(0,3)
$$

cuja parte divergente resulta em

$$
i \frac{1}{65536 \pi^{5}} \frac{\lambda^{3}}{\alpha^{5}} \frac{1}{d-10}
$$

\subsection{Equação do Grupo de Renormalização}

Seguindo o procedimento básico da renormalização [44, 45], interpretamos a lagrangiana inicial como a lagrangiana não-renormalizada, a qual, após uma reparametrização adequada, produz resultados finitos para as funções de vértice. Os parâmetros nãorenormalizados serão especificados pela letra $n$ e a lagrangiana não-renormalizada é

$$
\mathcal{L}=\frac{1}{2} \partial_{0} \varphi_{n} \partial_{0} \varphi_{n}-\frac{b_{n}^{2}}{2} \partial_{i} \varphi_{n} \partial_{i} \varphi_{n}-\frac{\alpha_{n}^{2}}{2} \varphi_{n} \Delta^{2} \varphi_{n}-\frac{m_{n}^{2}}{2} \varphi_{n}^{2}-\frac{\lambda_{n}}{3 !} \varphi_{n}^{3} .
$$

As quantidades renormalizadas e não-renormalizadas são relacionadas por meio das reparametrizações

$$
\varphi_{n}=Z_{\varphi}^{\frac{1}{2}} \varphi, m_{n}^{2}=m^{2}+\delta m^{2}, \quad b_{n}^{2}=\frac{Z_{b}}{Z_{\varphi}} b^{2}, \quad \alpha_{n}^{2}=\frac{Z_{\alpha}}{Z_{\varphi}} \alpha^{2} \text { and } \lambda_{n}=\frac{Z_{\lambda}}{Z_{\varphi}^{\frac{3}{2}}} \lambda .
$$

As quantidades $Z_{\varphi}, Z_{b}, Z_{\alpha}, Z_{\lambda}$ e $\delta m^{2}$ são escolhidas de modo a cancelar os termos divergentes das funções de Green de 2 e 3 pontos. Em termos das quantidades renormalizadas, a lagragiana torna-se

$$
\begin{aligned}
\mathcal{L} & =\frac{1}{2} \partial_{0} \varphi \partial_{0} \varphi-\frac{b^{2}}{2} \partial_{i} \varphi \partial_{i} \varphi-\frac{\alpha^{2}}{2} \varphi \Delta^{2} \varphi-\frac{m^{2}}{2} \varphi^{2}+\frac{1}{2}\left(Z_{\varphi}-1\right)\left(\partial_{0} \varphi \partial_{0} \varphi-m^{2} \varphi^{2}\right) \\
& -\frac{b^{2}}{2}\left(Z_{b}-1\right) \partial_{i} \varphi \partial_{i} \varphi-\frac{\delta m^{2} Z_{\varphi}}{2} \varphi^{2}-\frac{\alpha^{2}}{2}\left(Z_{\alpha}-1\right) \varphi \Delta^{2} \varphi-\frac{\lambda Z_{\lambda}}{3 !} \varphi^{3}
\end{aligned}
$$

O propagador renormalizado é

$$
\Delta(p)=\frac{i}{p_{0}^{2}-b^{2} \mathbf{p}^{2}-\alpha^{2}\left(\mathbf{p}^{2}\right)^{2}-m^{2}+i \epsilon}
$$

e as regras de Feynman para os vértices são

$$
\text { Vértice bilinear }=i\left(Z_{\varphi}-1\right)\left(p_{0}^{2}-m^{2}\right)-i b^{2}\left(Z_{b}-1\right) \mathbf{p}^{2}-i \delta m^{2} Z_{\varphi}-i\left(Z_{\alpha}-1\right) \alpha^{2}\left(\mathbf{p}^{2}\right)^{2}
$$


$\mathrm{e}$

$$
\text { Vértice trilinear }=-i \lambda Z_{\lambda} \text {. }
$$

Precisamos encontrar a relação entre os propagadores renormalizado e não-renormalizado. Usando as regras de Feynman acima, obtemos facilmente

$$
\Delta=Z_{\varphi}^{-1} \Delta_{n}
$$

Agora, podemos obter uma relação semelhante para as funções de vértice. A função de vértice de $N$ pontos renormalizada é definida como

$$
\begin{aligned}
& (2 \pi)^{d+1} \delta\left(p_{1}+p_{2}+\cdots+p_{N}\right) \Gamma^{(N)}\left(p_{1}, \cdots, p_{N}\right) \\
\equiv & \prod_{i=1}^{N} \Delta^{-1}\left(p_{i}\right) \int d^{D} x_{1} \cdots d^{D} x_{N}\left\langle 0\left|T \varphi\left(x_{1}\right) \cdots \varphi\left(x_{N}\right)\right| 0\right\rangle^{p r o p} e^{i \sum_{j=1}^{N} p_{j} x_{j}},
\end{aligned}
$$

em que prop significa a função de Green própria, incluindo apenas diagramas 1P1]. Por outro lado, a função não-renormalizada é

$$
\begin{aligned}
& (2 \pi)^{d+1} \delta\left(p_{1}+p_{2}+\cdots+p_{N}\right) \Gamma_{n}^{(N)}\left(p_{1}, \cdots, p_{N}\right) \\
\equiv & \prod_{i=1}^{N} \Delta_{n}^{-1}\left(p_{i}\right) \int d^{D} x_{1} \cdots d^{D} x_{N}\left\langle 0\left|T \varphi_{n}\left(x_{1}\right) \cdots \varphi_{n}\left(x_{N}\right)\right| 0\right\rangle^{p r o p} e^{i \sum_{j=1}^{N} p_{j} x_{j}} \\
= & (2 \pi)^{d+1} \delta\left(p_{1}+p_{2}+\cdots+p_{N}\right) Z_{\varphi}^{-\frac{N}{2}} \Gamma^{(N)}\left(p_{1}, \cdots, p_{N}\right) .
\end{aligned}
$$

Naturalmente, a função de vértice renormalizada dependerá de alguma escala de momento $\mu$ introduzida no processo de renormalização. É conveniente explicitar a dependência dos parâmetros nas funções de vértice, ou seja, $\Gamma_{n}^{(N)}\left(p, m_{n}^{2}, b_{n}^{2}, \alpha_{n}^{2}, \lambda_{n}\right)$ e $\Gamma^{(N)}\left(p, m^{2}, b^{2}, \alpha^{2}, \lambda, \mu\right)$, sendo que estamos representando todos os momentos externos compactamente por $p$. Dessa forma,

$$
0=\mu \frac{d}{d \mu} \Gamma_{n}^{(N)}\left(p, m_{n}^{2}, b_{n}^{2}, \alpha_{n}^{2}, \lambda_{n}\right)
$$

a qual, de acordo com (2.63), conduz à equação do grupo de renormalização de t'HooftWeinberg

$$
\left[\mu \frac{\partial}{\partial \mu}+\delta \frac{\partial}{\partial m^{2}}+\beta_{b^{2}} \frac{\partial}{\partial b^{2}}+\beta_{\alpha^{2}} \frac{\partial}{\partial \alpha^{2}}+\beta_{\lambda} \frac{\partial}{\partial \lambda}-N \gamma\right] \Gamma^{(N)}\left(p, m^{2}, b, \alpha, \lambda, \mu\right)=0,
$$

sendo que definimos,

$\mathrm{e}$

$$
\delta \equiv \mu \frac{\partial m^{2}}{\partial \mu}, \beta_{b^{2}} \equiv \mu \frac{\partial b^{2}}{\partial \mu}, \beta_{\alpha^{2}} \equiv \mu \frac{\partial \alpha^{2}}{\partial \mu}, \quad \beta_{\lambda} \equiv \mu \frac{\partial \lambda}{\partial \mu}
$$

$$
\gamma \equiv \frac{\mu}{2} \frac{1}{Z_{\varphi}} \frac{\partial Z_{\varphi}}{\partial \mu}=\frac{\mu}{2} \frac{\partial}{\partial \mu} \ln Z_{\varphi}
$$

\footnotetext{
${ }^{1}$ Diagramas 1PI são aqueles que não podem ser separados em duas partes cortando-se apenas uma linha interna.
} 


\subsection{Funções do Grupo de Renormalização}

No contexto da regularização dimensional, o parâmetro dimensional $\mu$ é introduzido de modo que a constante de acoplamento mantenha-se adimensional mesmo quando estamos numa dimensão arbitrária, isto é, fora da dimensão física $d=10$. Isso pode ser obtido por meio de

$$
\lambda \rightarrow \lambda \mu^{\frac{\epsilon}{2}}
$$

em que $\epsilon \equiv 10-d$. Então, o termo de interação cúbico da lagrangiana torna-se

$$
\mathcal{L}_{\text {int }}=-\frac{\lambda}{3 !} \mu^{\frac{\epsilon}{2}} \varphi^{3}
$$

\subsubsection{Função de 2 pontos}

Inicialmente, vamos nos concentrar na função de 2 pontos. Podemos escrever,

$$
\Gamma^{(2)}=i\left[p_{0}^{2}-b^{2} \mathbf{p}^{2}-\alpha^{2}\left(\mathbf{p}^{2}\right)^{2}-m^{2}+\Sigma\right],
$$

em que $\Sigma$ representa a correção do diagrama de um laço mostrado na figura 2.1 e possui a estrutura

$$
\left.\Sigma=-\lambda^{2} \mu^{\epsilon} \text { (Parte finita } 1+\text { Pólo }_{1}\right) .
$$

Note que, $\mu^{\epsilon}=e^{\epsilon \ln \mu}=1+\epsilon \ln \mu+O\left(\epsilon^{2}\right)$. Assim, obtemos

$$
\Sigma=-\lambda^{2}\left(\text { Parte finita }_{1}-\ln \mu \text { Resíduo }_{1}+\text { Pólo }_{1}\right) .
$$

É importante observar a mudança de sinal na multiplicação $\epsilon \ln \mu \mathrm{Pólo}_{1}=-\ln \mu$ Resíduo $_{1}$, pois o pólo é caracterizado pelo fator $(d-10)^{-1}$. A função de vértice renormalizada pode ser pensada como resultante de (2.70), depois da aplicação do operador de subtração de pólos, ou seja,

$$
\Gamma^{(2)}=i\left[p_{0}^{2}-b^{2} \mathbf{p}^{2}-\alpha^{2}\left(\mathbf{p}^{2}\right)^{2}-m^{2}-\lambda^{2}\left(\text { Parte finita }_{1}-\ln \mu \text { Resíduo }_{1}\right)\right] .
$$

A estrutura geral do resíduo é

$$
\text { Resíduo }_{1}=a_{1}+a_{2} p_{0}^{2}+a_{3} \mathbf{p}^{2}+a_{4}\left(\mathbf{p}^{2}\right)^{2} .
$$

Os fatores $a_{i}$, podem ser lidos diretamente das equações (2.39), (2.43), (2.46), (2.49), fornecendo,

$$
\begin{gathered}
a_{1}=\frac{1}{262144 \pi^{5}} \frac{5 b^{4}-4 \alpha^{2} m^{2}}{\alpha^{7}}, \quad a_{2}=\frac{1}{393216 \pi^{5}} \frac{1}{\alpha^{5}} \\
a_{3}=\frac{5}{393216 \pi^{5}} \frac{b^{2}}{\alpha^{5}}, \quad a_{4}=\frac{11}{3932160 \pi^{5}} \frac{1}{\alpha^{3}} .
\end{gathered}
$$


Agora, devemos substituir (2.73) na equação do grupo de renormalização (2.65),

$$
\begin{aligned}
& \lambda^{2} \text { Resíduo }_{1}+\delta\left[-1-\lambda^{2}\left(\frac{\partial}{\partial m^{2}} \text { Parte finita }_{1}-a_{1} \ln \mu\right)\right] \\
+ & \beta_{b^{2}}\left[-\mathbf{p}^{2}-\lambda^{2}\left(\frac{\partial}{\partial b^{2}} \text { Parte finita }_{1}-\ln \mu \frac{\partial}{\partial b^{2}} \text { Resíduo }_{1}\right)\right] \\
+ & \beta_{\alpha^{2}}\left[-\left(\mathbf{p}^{2}\right)^{2}-\lambda^{2}\left(\frac{\partial}{\partial \alpha^{2}} \text { Parte finita }_{1}-\ln \mu \frac{\partial}{\partial \alpha^{2}} \text { Resíduo }_{1}\right)\right] \\
+ & \beta_{\lambda}\left[-2 \lambda\left(\text { Parte finita }_{1}-\ln \mu \text { Resíduo }_{1}\right)\right] \\
- & 2 \gamma\left[p_{0}^{2}-b^{2} \mathbf{p}^{2}-\alpha^{2}\left(\mathbf{p}^{2}\right)^{2}-m^{2}-\lambda^{2}\left(\text { Parte finita }_{1}-\ln \mu \text { Resíduo }_{1}\right)\right]=0 .
\end{aligned}
$$

Essa equação, juntamente com a equação correspondente vinda da função de 3 pontos, que será considerada na sequência, podem ser resolvidas perturbativamente como faremos em breve.

\subsubsection{Função de 3 pontos}

Uma análise semelhante a anterior, conduz à função de 3 pontos renormalizada. Um ponto que merece destaque refere-se ao fator $\mu^{\frac{\epsilon}{2}}$ que devemos extrair para manter inalterada a dimensão da função de 3 pontos,

$$
\Gamma^{(3)}=\mu^{\frac{\epsilon}{2}}\left[-i \lambda+i \lambda^{3} \mu^{\epsilon}\left(\text { Parte finita } 2+\text { Pólo }_{2}\right)\right] .
$$

Portanto, a função renormalizada será,

$$
\Gamma^{(3)}=-i \lambda+i \lambda^{3}\left(\text { Parte finita }_{2}-\ln \mu \text { Resíduo }_{2}\right) .
$$

Esse resíduo tem a estrutura simples

$$
\text { Resíduo }_{2}=b_{1},
$$

em que $b_{1}$ pode ser lido em (2.54),

$$
b_{1}=\frac{1}{65536 \pi^{5}} \frac{1}{\alpha^{5}} .
$$

Substituindo (2.79) em (2.65), obtemos

$$
\begin{aligned}
& -\lambda^{3} \text { Resíduo }_{2}+\lambda^{3} \delta\left(\frac{\partial}{\partial m^{2}} \text { Parte finita }_{2}-\ln \mu \frac{\partial}{\partial m^{2}} \text { Resíduo }_{2}\right) \\
+ & \lambda^{3} \beta_{b^{2}}\left(\frac{\partial}{\partial b^{2}} \text { Parte finita }_{2}-\ln \mu \frac{\partial}{\partial b^{2}} \text { Resíduo }_{2}\right) \\
+ & \lambda^{3} \beta_{\alpha^{2}}\left(\frac{\partial}{\partial \alpha^{2}} \text { Parte finita }_{2}-\ln \mu \frac{\partial}{\partial \alpha^{2}} \text { Resíduo }_{2}\right) \\
+ & \beta_{\lambda}\left[-1+3 \lambda^{2}\left(\text { Parte finita }_{2}-\ln \mu \text { Resíduo }_{2}\right)\right] \\
- & 3 \gamma\left[-\lambda+\lambda^{3}\left(\text { Parte finita }_{2}-\ln \mu \text { Resíduo }_{2}\right)\right]=0 .
\end{aligned}
$$




\subsection{Solução Perturbativa}

Vamos estudar a solução perturbativa das equações acima. A idéia básica é desenvolver todas as funções em séries de potências da constante de acoplamento $\lambda$. A utilização do esquema de subtração minimal dos pólos garante que tais funções não dependam explicitamente de razões de massas $(\mu / m)$, dependendo apenas da constante de acoplamento:

$$
\begin{gathered}
\delta=\delta^{(1)}+\delta^{(2)}+\delta^{(3)}+\cdots, \\
\beta_{b^{2}}=\beta_{b^{2}}^{(1)}+\beta_{b^{2}}^{(2)}+\beta_{b^{2}}^{(3)}+\cdots, \\
\beta_{\alpha^{2}}=\beta_{\alpha^{2}}^{(1)}+\beta_{\alpha^{2}}^{(2)}+\beta_{\alpha^{2}}^{(3)}+\cdots, \\
\beta_{\lambda}=\beta_{\lambda}^{(1)}+\beta_{\lambda}^{(2)}+\beta_{\lambda}^{(3)}+\cdots
\end{gathered}
$$

e

$$
\gamma=\gamma^{(1)}+\gamma^{(2)}+\gamma^{(3)}+\cdots,
$$

em que o índice superior designa a ordem correspondente na constante de acoplamento. Levando essas expansões em (2.77) and (2.82), obtemos até a primeira ordem na constante de acoplamento,

$$
\delta^{(1)}=\beta_{b^{2}}^{(1)}=\beta_{\alpha^{2}}^{(1)}=\beta_{\lambda}^{(1)}=\gamma^{(1)}=0,
$$

como esperado. As relações de segunda ordem são

$$
\begin{gathered}
\lambda^{2} a_{1}-\delta^{(2)}+2 \gamma^{(2)} m^{2}=0, \\
\lambda^{2} a_{2}-2 \gamma^{(2)}=0, \\
\lambda^{2} a_{3}-\beta_{b^{2}}+2 b^{2} \gamma^{(2)}=0, \\
\lambda^{2} a_{4}-\beta_{\alpha^{2}}^{(2)}+2 \alpha^{2} \gamma^{(2)}=0
\end{gathered}
$$

e

$$
\beta_{\lambda}^{(2)}=0 .
$$

A equação de terceira ordem é

$$
-b_{1} \lambda^{3}-\beta_{\lambda}^{(3)}+3 \gamma^{(2)} \lambda=0 .
$$

As demais funções são nulas nessa ordem,

$$
\delta^{(3)}=\beta_{b^{2}}^{(3)}=\beta_{\alpha^{2}}^{(3)}=\gamma^{(3)}=0 .
$$

Com essas relações, estamos prontos para analisar o fluxo do grupo de renormalização. A função $\gamma^{(2)}$ é dada diretamente por (2.90):

$$
\gamma^{(2)}=\frac{a_{2}}{2} \lambda^{2}=\frac{1}{786432 \pi^{5}} \frac{\lambda^{2}}{\alpha^{5}} .
$$


Daí, podemos obter as outras funções,

$$
\begin{gathered}
\delta^{(2)}=\left(a_{1}+m^{2} a_{2}\right) \lambda^{2}=\frac{\left(5 b^{4}-\frac{10}{3} m^{2} \alpha^{2}\right)}{262144 \pi^{5}} \frac{\lambda^{2}}{\alpha^{7}}, \\
\beta_{b^{2}}^{(2)}=\left(a_{3}+b^{2} a_{2}\right) \lambda^{2}=\frac{b^{2}}{65536 \pi^{5}} \frac{\lambda^{2}}{\alpha^{5}}, \\
\beta_{\alpha^{2}}^{(2)}=\left(a_{2} \alpha^{2}+a_{4}\right) \lambda^{2}=\frac{7}{1310720 \pi^{5}} \frac{\lambda^{2}}{\alpha^{3}}
\end{gathered}
$$

$\mathrm{e}$

$$
\beta_{\lambda}^{(3)}=\left(\frac{3}{2} a_{2}-b_{1}\right) \lambda^{3}=-\frac{3}{262144 \pi^{5}} \frac{\lambda^{3}}{\alpha^{5}} .
$$

Esses resultados também foram obtidos em [34]. A função $\beta_{\lambda}^{(3)}$ mostra que a teoria é assintoticamente livre, tal como no caso usual (compare com (2.13)). Portanto, a liberdade assintótica da teoria não é afetada pela introdução da anisotropia para esse modelo simples. Além disso, (2.97) define uma relação crítica entre os parâmetros

$$
b_{c}^{4}=\frac{2}{3} m_{c}^{2} \alpha_{c}^{2}
$$

e, dependendo se $b^{4}>\frac{2}{3} m^{2} \alpha^{2}$ ou $b^{4}<\frac{2}{3} m^{2} \alpha^{2}$, a massa efetiva muda seu comportamento mediante o fluxo do grupo de renormalização.

Antes de prosseguir com a solução das equações, devemos fazer alguns comentários sobre a possibilidade da restauração da simetria de Lorentz. Considerando inicialmente a teoria livre, a invariância de Lorentz requer $\alpha=0$. Por outro lado, ao introduzir a interação, as funções de Green tornam-se divergentes e a renormalizabilidade depende de $\alpha$ ser diferente de zero, ou seja, na dimensão considerada, $d=10$, o modelo é renormalizável apenas se o termo com derivadas de ordem superior estiver presente. Como reflexo disso, note que as equações acima divergem quando $\alpha=0$. Portanto, devemos falar da simetria de Lorentz em um sentido aproximado. Dado um conjunto de valores iniciais dos parâmetros da teoria, esperamos que o fluxo do grupo de renormalização conduza os parâmetros a certas configurações em baixas energias tal que o termo de quebra seja pequeno e o sistema exiba um comportamento relativístico efetivo.

Apesar de não ser possível fazer $\alpha=0$ quando há interação, isto é, quando $\lambda \neq 0$, poderíamos ter uma situação interessante tomando os limites $\alpha \rightarrow 0$ e $\lambda \rightarrow 0$ simultaneamente, mas com uma razão apropriada entre esses parâmetros mantida fixa. Isso define um parâmetro adequado para se fazer a teoria de perturbação, sendo dado por

$$
\lambda_{e f f}^{2} \equiv \frac{\lambda^{2}}{\alpha^{5}}
$$

que desempenha o papel de uma constante de acoplamento efetiva. Essa razão é definida a partir da observação que as equações (2.98), (2.99) e (2.100) são todas da forma

$$
\beta_{(\mathrm{P})}=(\cdots)(\mathrm{P}) \lambda_{e f f}^{2},
$$


sendo que $(\cdots)$ representa fatores numéricos e $(\mathrm{P})=b^{2}, \alpha^{2}$ ou $\lambda$. Como $\alpha$ e $\lambda$ têm comportamentos opostos mediante o fluxo do grupo de renormalização, certamente essa relação não se manterá estável. Para ver isso precisamente, devemos investigar as soluções das equações (2.99) e (2.100).

Vamos resolver o conjunto de equações diferenciais acopladas para obter os parâmetros efetivos. Inicialmente, vamos nos concentrar em (2.99) e (2.100). Introduzindo uma escala logaritmica, $t \equiv \ln \mu / \mu_{0}$, em que $\mu_{0}$ é a escala de energia em que os parâmetros efetivos tornam-se iguais aos originais, temos

$$
\frac{\partial \bar{\alpha}^{2}}{\partial t}=\frac{7}{1310720 \pi^{5}} \frac{\bar{\lambda}^{2}}{\bar{\alpha}^{3}}
$$

$\mathrm{e}$

$$
\frac{\partial \bar{\lambda}}{\partial t}=-\frac{3}{262144 \pi^{5}} \frac{\bar{\lambda}^{3}}{\bar{\alpha}^{5}},
$$

sob as condições iniciais $\bar{\alpha}(0)=\alpha$ e $\bar{\lambda}(0)=\lambda$. Note que, podemos escrever (2.104) como

$$
\frac{2}{5} \frac{\partial \bar{\alpha}^{5}}{\partial t}=\frac{7}{1310720 \pi^{5}} \bar{\lambda}^{2}
$$

Assim, substituindo (2.105) no lado esquerdo dessa equação, obtemos uma equação diferencial de segunda ordem não-linear, evolvendo apenas $\bar{\lambda}(t)$,

$$
\bar{\lambda} \frac{\partial^{2} \bar{\lambda}}{\partial t^{2}}=\frac{25}{6}\left(\frac{\partial \bar{\lambda}}{\partial t}\right)^{2} .
$$

A solução geral é

$$
\bar{\lambda}(t)=\frac{c_{2}}{\left(19 t+6 c_{1}\right)^{\frac{6}{19}}}
$$

em que $c_{1}$ e $c_{2}$ são constantes a serem determinadas. Com essa solução, podemos facilmente obter $\bar{\alpha}(t)$ a partir de (2.106)

$$
\bar{\alpha}^{5}(t)=\frac{c_{2}^{2}}{524288 \pi^{5}}\left(19 t+6 c_{1}\right)^{\frac{7}{19}} .
$$

Aplicando as condições iniciais, $\bar{\alpha}(0)=\alpha$ e $\bar{\lambda}(0)=\lambda$, temos

$$
c_{1}=\frac{262144 \pi^{5}}{3} \frac{\alpha^{5}}{\lambda^{2}}
$$

$\mathrm{e}$

$$
c_{2}=64 \pi^{\frac{30}{19}} \alpha^{\frac{30}{19}} \lambda^{\frac{7}{19}}
$$

Substituindo as soluções (2.108) e (2.109) em (2.98), obtemos o parâmetro efetivo $\bar{b}(t)$,

$$
\bar{b}^{2}(t)=\frac{b^{2}}{\left(6 c_{1}\right)^{\frac{8}{19}}}\left(19 t+6 c_{1}\right)^{\frac{8}{19}}
$$


sendo que, levamos em conta a condição inicial $\bar{b}(0)=b$.

A partir das soluções acima, vemos que existe um valor crítico de $t, t_{I R}$, tal que, para valores de $t$ menores que $t_{I R}$, os parâmetros da teoria tornam-se complexos e a mesma não faz mais sentido. Na verdade, mesmo antes de atingir esse valor crítico, a constante de acoplamento cresce, de modo que a própria teoria de perturbação não se justifica. Esse valor crítico fornece um limite infravermelho e é dado por

$$
t_{I R}=-\frac{524288 \pi^{5}}{19} \frac{\alpha^{5}}{\lambda^{2}}
$$

Assim, quando $t \rightarrow t_{I R}$, temos $\bar{\alpha} \rightarrow 0, \bar{g} \rightarrow \infty$ e $\bar{b} \rightarrow 0$.

Por fim, usando as soluções (2.108) e (2.109), podemos determinar $\bar{\lambda}_{\text {eff }}(t)$ de acordo com (2.102):

$$
\bar{\lambda}_{e f f}^{2}(t)=\frac{\lambda_{e f f}^{2}}{1+\frac{19}{524288 \pi^{5}} \lambda_{e f f}^{2} t},
$$

mostrando que a razão $\lambda^{2} / \alpha^{5}$ não é estável no infravermelho, divergindo no ponto $t_{I R}$.

Como uma lição geral, podemos dizer que devido à liberdade assintótica da teoria, não podemos alcançar um limite de energia tão baixo quanto desejamos, pois a própria teoria de perturbação deixa de fazer sentido. Isso impõe um grande obstáculo para os nossos propósitos de estudar os limites de baixas energias. Esperamos que, qualitativamente, esse tipo de comportamento seja compartilhado com outras teorias de maior interesse, como o caso de Yang-Mills e o modelo sigma não-linear, que também são assintoticamente livres. De qualquer forma, o estudo de modelos estáveis no infravermelho pode proporcionar uma situação mais favorável.

\subsection{Modelo $\varphi^{4}$}

De acordo com a discussão anterior, teorias fracamente acopladas no infravermelho são mais apropriadas para o tipo de análise que desejamos realizar, pois podemos acessar limites de baixas energias dentro do esquema perturbativo. Vamos considerar um modelo escalar com interação $\varphi^{4}$ que no caso usual não é assintoticamente livre, com a correspondente função beta

$$
\beta_{\lambda}=\frac{3}{16 \pi^{2}} \lambda^{2}
$$

A lagrangiana para o caso anisotrópico com $z=2$ é dada por

$$
\mathcal{L}=\frac{1}{2} \partial_{0} \varphi \partial_{0} \varphi-\frac{b^{2}}{2} \partial_{i} \varphi \partial_{i} \varphi-\frac{\alpha^{2}}{2} \varphi \Delta^{2} \varphi-\frac{m^{2}}{2} \varphi^{2}-\frac{\lambda}{4 !} \varphi^{4} .
$$

Esse modelo também é de grande relevância na matéria condensada, constituindo um protótipo usado para a descrição geral de transições de fase de acordo com a expansão de 
Landau, para teorias exibindo um ponto de Lifshitz [17]. A teoria é renormalizável em $d=6$ dimensões espaciais, com grau de divergência superficial

$$
d(G)=8-2 N
$$

As funções de vértice divegentes são aquelas com $N=2$ e $N=4$, com divergência superficial $d(G)=4$ e $d(G)=0$, respectivamente. Os diagramas relevantes são mostrados nas figuras 2.3, 2.4 e 2.5. A expressão associada ao diagrama da figura 2.3 é

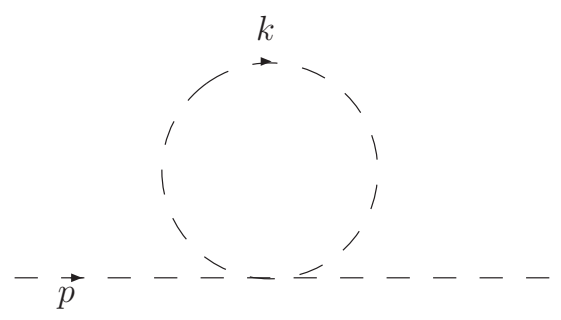

Figura 2.3: Função de 2 pontos da ordem $\lambda$.

$$
\frac{\lambda}{2} \int \frac{d k_{0}}{2 \pi} \frac{d^{d} k}{(2 \pi)^{d}} \frac{1}{k_{0}^{2}-b^{2} \mathbf{k}^{2}-\alpha^{2}\left(\mathbf{k}^{2}\right)^{2}-m^{2}+i \epsilon} .
$$

Em princípio, devemos aplicar um operador de Taylor de quarta ordem, $t^{4}$, para revelar os termos de pólo. Entretanto, como o diagrama não depende do momento externo, apenas o termo sem derivadas sobrevive. Podemos usar (2.16) e (2.24) para identificar

$$
\frac{\lambda}{2} f(d) g(x, y)
$$

O cálculo da parte divergente fornece

$$
-i \frac{\left(-3 b^{4}+4 \alpha^{2} m^{2}\right)}{2048 \pi^{3}} \frac{\lambda}{\alpha^{5}} \frac{1}{d-6} .
$$

Vemos daí que esse gráfico é responsável por uma correção apenas para o termo de massa. Para obter correções para os termos cinéticos, devemos considerar o gráfico da ordem seguinte, correspondente ao diagrama de dois laços da figura 2.4. Esse diagrama proporcionará correções para os parâmetros de maior interesse, $b$ and $\alpha$. No entanto, a extração de sua parte divergente não é uma tarefa simples e parece ser possível apenas em casos especiais, conforme discutiremos. Pelo menos, é possível determinar a função beta associada à constante de acomplamento $\lambda$. Para isso, devemos considerar a função de 4 pontos. Como a divergência é logaritmica, para extrair sua parte divergente basta calcular o diagrama com os momentos externos iguais a zero. Levando em conta a contribuição dos três canais de Mandelstam, temos

$$
\frac{3 \lambda^{2}}{2} \int \frac{d k_{0}}{2 \pi} \frac{d^{d} k}{(2 \pi)^{d}} \frac{1}{\left[k_{0}^{2}-b^{2} \mathbf{k}^{2}-\alpha^{2}\left(\mathbf{k}^{2}\right)^{2}-m^{2}+i \epsilon\right]^{2}} .
$$




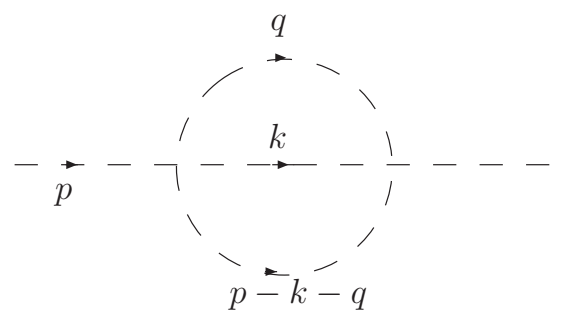

Figura 2.4: Função de 2 pontos da ordem $\lambda^{2}$.

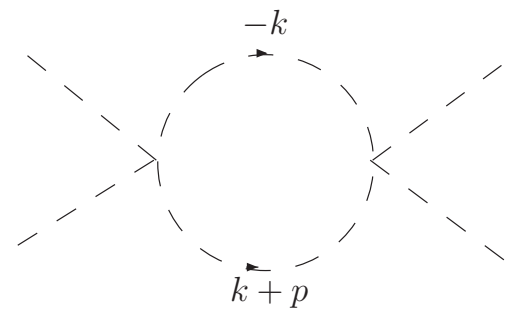

Figura 2.5: Função de 4 pontos.

Novamente, podemos identificar essa expressão com (2.16) e (2.24),

$$
\frac{3 \lambda^{2}}{2} f(d) g(0,2)
$$

tal que a parte divergente resulta em

$$
-i \frac{3}{512 \pi^{3}} \frac{\lambda^{2}}{\alpha^{3}} \frac{1}{d-6}
$$

Agora, procedemos com o estudo do grupo de renormalização.

\subsubsection{Grupo de Renormalização}

A análise do grupo de renormalização é completamente semelhante a do modelo $\varphi^{3}$ e por isso a apresentaremos de uma maneira sucinta. Primeiramente, o parâmetro $\mu$ é introduzido da maneira usual,

$$
\lambda \rightarrow \mu^{\epsilon} \lambda,
$$

em que agora $\epsilon=6-d$. A função de vértice de 2 pontos renormalizada é

$$
\Gamma^{(2)}=i\left[p_{0}^{2}-b^{2} \mathbf{p}^{2}-\alpha^{2}\left(\mathbf{p}^{2}\right)^{2}-m^{2}-\lambda\left(\text { Parte finita }_{1}-\ln \mu \text { Resíduo }_{1}\right)\right] .
$$

Relembre que estamos levando em conta apenas a contribuição de primeira ordem na constante de acoplamento, correspondente ao diagrama 2.3. O resíduo é uma constante 
em relação ao momento, ou seja, tem a estrutura Resíduo ${ }_{1}=a_{1}$, e $a_{1}$ pode ser lida diretamente de (2.120):

$$
a_{1}=-i \frac{\left(-3 b^{4}+4 \alpha^{2} m^{2}\right)}{2048 \pi^{3}} \frac{1}{\alpha^{5}} .
$$

Para a função de 4 pontos renormalizada, temos

$$
\Gamma^{(4)}=-i \lambda-\lambda^{2}\left(\text { Parte finita }_{2}-\ln \mu \text { Resíduo }_{2}\right),
$$

com o resíduo da forma, Resíduo $2=c_{1}$, e $c_{1}$ obtida de (2.123):

$$
c_{1}=i \frac{3}{512 \pi^{3}} \frac{1}{\alpha^{3}}
$$

Substituindo as funções de vértice nas equações do grupo de renormalização correspondentes e considerando expansões tais como em (2.83) até (2.87), obtemos

$$
\delta^{(1)}=i a_{1} \lambda=\frac{\left(-3 b^{4}+4 \alpha^{2} m^{2}\right)}{2048 \pi^{3}} \frac{\lambda}{\alpha^{5}}
$$

e a função beta

$$
\beta_{\lambda}^{(2)}=-i c_{1} \lambda^{2}=\frac{3}{512 \pi^{3}} \frac{\lambda^{2}}{\alpha^{3}} .
$$

Assim como no modelo $\varphi^{3}$, a equação de evolução da massa (2.129) define uma relação crítica $b_{c}^{4}=\frac{4}{3} m_{c}^{2} \alpha_{c}^{2}$. A equação (2.130), por sua vez, mostra que a teoria é estável no infravermelho, o que é desejável para nossas propostas. Entretanto, não temos informações sobre os fluxos de $b$ ou $\alpha$. Nesse caso, é instrutivo considerar alguma situação especial, em que seja possível prosseguir com análise de pelo menos algum deles.

\subsubsection{Teoria com Invariância de Escala Clássica}

Quando os parâmetros dimensionais são tomados iguais a zero, no nosso caso, $b$ e $m$, a teoria torna-se invariante classicamente por transformações de escala anisotrópica $x^{0} \rightarrow l^{z} x^{0}$ e $x^{i} \rightarrow l x^{i}$. Quanticamente, essa simetria é quebrada devido a uma anomalia na corrente de dilatação, refletindo a necessidade da introdução de um parâmetro dimensional no processo de renormalização. Voltaremos a esse ponto no capítulo 4, em que discutiremos a construção de correntes quânticas e, em particular, a corrente associada às transformações de escala. No presente caso com $b=0$ e $m=0$ é possível determinar a parte divergente do diagrama de dois laços (figura 2.4) e consequentemente analisar o fluxo do parâmetro $\alpha$. A lagrangiana torna-se

$$
\mathcal{L}=\frac{1}{2} \partial_{0} \varphi \partial_{0} \varphi-\frac{\alpha^{2}}{2} \varphi \Delta^{2} \varphi-\frac{\lambda}{4 !} \varphi^{4}
$$


com o propagador

$$
\Delta(k)=\frac{i}{k_{0}^{2}-\alpha^{2}\left(\mathbf{k}^{2}\right)^{2}+i \epsilon} .
$$

Vamos nos concentrar no diagrama de dois laços da figura 2.4. A expressão correspondente envolve uma integração sobre dois momentos internos, dada por

$$
\frac{i \lambda^{2}}{3 !} \int \frac{d k_{0}}{2 \pi} \frac{d^{d} k}{(2 \pi)^{d}} \frac{d q_{0}}{2 \pi} \frac{d^{d} q}{(2 \pi)^{d}} \frac{1}{k_{0}^{2}-\alpha^{2}\left(\mathbf{k}^{2}\right)^{2}} \frac{1}{q_{0}^{2}-\alpha^{2}\left(\mathbf{q}^{2}\right)^{2}} \frac{1}{\left(p_{0}-k_{0}-q_{0}\right)^{2}-\alpha^{2}\left((\mathbf{p}-\mathbf{k}-\mathbf{q})^{2}\right)^{2}} .
$$

Por simplicidade estamos omitindo a prescrição $i \epsilon$. Esse tipo de integral foi estudada em detalhes no contexto da matéria condensada na referência [46]. De acordo com os resultados lá apresentados, a parte divergente é

$$
-\frac{i \lambda^{2}}{288} \frac{\pi^{2}}{\alpha^{4}}\left[\frac{1}{\alpha^{2}}\left(1+3 \ln \frac{3}{4}\right) p_{0}^{2}+\frac{7}{72}\left(\mathbf{p}^{2}\right)^{2}\right] \frac{1}{d-6} .
$$

Por razões dimensionais, o diagrama da figura 2.3 não tem contribuição divergente, o que pode ser visto também a partir de (2.120). Por outro lado, a parte divergente da função de 4 pontos, correspondente ao diagrama 2.5, não é alterada quando tomamos $b=m=0$ (veja (2.123) ).

Depois da análise do grupo de renormalização, obtemos

$$
\beta_{\alpha^{2}}^{(2)}=\frac{\pi^{2}}{144}\left(\frac{79}{72}+3 \ln \frac{3}{4}\right) \frac{\lambda^{2}}{\alpha^{4}} \approx 0,23 \frac{\pi^{2}}{144} \frac{\lambda^{2}}{\alpha^{4}},
$$

mostrando que $\alpha(t)$ cresce no ultravioleta. A função beta é aquela dada em (2.130);

$$
\beta_{\lambda}^{(2)}=\frac{3}{512 \pi^{3}} \frac{\lambda^{2}}{\alpha^{3}}
$$

Quanto à solução dessas equações, há uma configuração particular dos valores iniciais dos parâmetros $\alpha$ e $\lambda$, tal que o sistema de equações diferenciais exibe uma solução analítica simples. De fato, definindo as constantes $A \equiv \frac{\pi^{2}}{144}\left(\frac{79}{72}+3 \ln \frac{3}{4}\right)$ e $B \equiv \frac{3}{512 \pi^{3}}$, obtemos a partir (2.135) e (2.136), a seguinte equação para a constante de acoplamento efetiva

$$
\frac{3 A}{2 B^{2}}\left(\frac{\partial \bar{\lambda}}{\partial t}\right)^{3}-2 \bar{\lambda}\left(\frac{\partial \bar{\lambda}}{\partial t}\right)^{2}+\bar{\lambda}^{2}\left(\frac{\partial^{2} \bar{\lambda}}{\partial t^{2}}\right)=0
$$

a qual admite a solução

$$
\bar{\lambda}(t)=\bar{\lambda}(0) e^{\frac{3 A}{2 B^{2}} t}
$$

Assim, de (2.135), obtemos

$$
\bar{\alpha}(t)=\left[\frac{9 A^{2}}{4 B^{2}} \bar{\lambda}^{2}(0)\left(e^{\frac{3 A}{B^{2}} t}-1\right)+\bar{\alpha}^{6}(0)\right]^{\frac{1}{6}} .
$$


Mas essa apenas será solução do sistema sob a condição $\bar{\alpha}^{6}(0) \equiv \frac{9 A^{2}}{4 B^{2}} \bar{\lambda}^{2}(0)$, conduzindo a

$$
\bar{\alpha}(t)=\left(\frac{9 A^{2}}{4 B^{2}} \bar{\lambda}^{2}(0)\right)^{\frac{1}{6}} e^{\frac{A}{2 B^{2}} t}
$$

Esse resultado mostra que o parâmetro de quebra da simetria de Lorentz $\bar{\alpha}$, assim como a constante de acoplamento tendem para zero quando $t \rightarrow-\infty$, na região do infravermelho. Por análise dimensional, vemos que essas soluções não seriam modificadas mesmo na presença dos parâmetros $b$ e $m$, tal como na lagrangiana (2.116). De forma heurística, podemos obter então uma situação em que a simetria de Lorentz se manifeste num limite de energia suficientemente baixo. Para concretizar essa possibilidade, devemos considerar a constante de acoplamente efetiva, que nesse case é definida como

$$
\lambda_{e f f} \equiv \frac{\lambda}{\alpha^{3}}
$$

Assim, podemos determinar $\bar{\lambda}_{e f f}(t)$ por meio das soluções (2.138) e (2.140), cujo resultado é

$$
\bar{\lambda}_{e f f}(t)=0,0078493
$$

independente da escala $t$ ! Isso é muito interessante, pois significa que podemos tomar $\alpha$ e $\lambda$ indo para zero, mantendo fixa a razão (2.141), e essa razão permanecerá fixa qualquer que seja a escala. Logo, esse é um parâmetro muito adequado para se fazer a teoria de perturbação. Como esse resultado não seria modificado mesmo na presença dos parâmetros dimensionais $b$ e $m$, concluímos que a ação efetiva de baixas energias para o modelo anisotrópico (2.116) deve ter a forma

$$
S \sim \int_{I R} d x^{0} d^{6} x\left(\frac{1}{2} \partial_{0} \varphi \partial_{0} \varphi-\frac{b_{e f f}^{2}}{2} \partial_{i} \varphi \partial_{i} \varphi-\frac{m_{e f f}^{2}}{2} \varphi^{2}-\frac{\lambda_{e f f}}{4 !} \varphi^{4}\right)
$$

em que $b_{\text {eff }}$ e $m_{\text {eff }}$ designam valores de baixas energias dos parâmetros $b$ e $m$. O subscrito $I R$ significa que a integração deve ser restrita à região de baixas energias, ou seja, acima de uma determinada escala de comprimento $l:|x|>l$. Com um reescalonamento da coordenada espacial podemos eliminar $b_{\text {eff }}$ e obtemos então temos uma teoria com a invariância de Lorentz! 


\section{Capítulo 3}

\section{Grupo de Renormalização - Modelo de Yukawa}

A construção de teorias com mais de um campo interagindo envolve um número maior de parâmetros e isso torna o estudo sobre a possibilidade de restauração da simetria de Lorentz mais complicado. Neste capítulo, discutimos a questão da anisotropia no contexto de teorias envolvendo campos fermiônicos e bosônicos e prosseguimos na investigação do grupo de renormalização considerando o modelo de Yukawa com $z=2$ [42].

\subsection{Considerações Gerais e Contagem de Potências}

Antes de partir propriamente para o estudo do modelo de Yukawa, vamos retomar à discussão feita no primeiro capítulo e elaborar um pouco mais. A forma geral da parte livre de um campo espinorial com grau de anisotropia $z$ é

$$
\mathcal{L}_{0}=\bar{\psi} i \gamma^{0} \partial_{0} \psi+\bar{\psi}\left(\alpha_{1} i \gamma^{i} \partial_{i}+\alpha_{2}\left(i \gamma^{i} \partial_{i}\right)^{2}+\cdots+\alpha_{z}\left(i \gamma^{i} \partial_{i}\right)^{z}-M\right) \psi,
$$

sendo $\psi$ um espinor de Dirac, com $\bar{\psi} \equiv \psi^{\dagger} \gamma^{0}$, e as matrizes $\gamma^{\mu}$ satisfazendo a álgebra:

$$
\left\{\gamma^{\mu}, \gamma^{\nu}\right\}=2 g^{\mu \nu} .
$$

O propagador correspondente à lagrangiana (3.1) é dado por

$$
S(k)=\frac{i}{\gamma^{0} k^{0}-\alpha_{1} \gamma \cdot \mathbf{k}+\cdots+\alpha_{z}(-\gamma \cdot \mathbf{k})^{z}-M},
$$

em que $\gamma \cdot \mathbf{k} \equiv \gamma^{i} k^{i}$. Como já discutimos, os termos relevantes para a contagem de potências da teoria são o da derivada temporal e o da derivada espacial de ordem mais alta. 
Podemos facilmente generalizar a expressão (2.7) para incluir campos fermiônicos. Dado um diagrama arbitrário $G$, com $L$ laços, $n_{B}$ linhas internas bosônicas e $n_{F}$ linhas internas fermiônicas, o grau de divergêncial superficial de $G$ é

$$
d(G)=(d+z) L-2 z n_{B}-z n_{F} .
$$

Note que o grau de anisotropia $z$ deve ser o mesmo para os campos escalar e espinorial. Agora, usando a relação de Euler, $L=n_{B}+n_{F}-V+1$, em que $V$ é o número de vértices, e as identidades topológicas

$$
2 n_{B}+N_{B}=\sum_{a} \nu_{a}^{B} \quad \text { e } \quad 2 n_{F}+N_{F}=\sum_{a} \nu_{a}^{F}
$$

em que $\nu_{a}^{B}$ e $\nu_{a}^{F}$ são os números de linhas bosônicas e fermiônicas juntando-se no vértice $a$, e $N_{B}$ e $N_{F}$ são linhas externas bosônicas e fermiônicas, obtemos

$$
d(G)=d+z-\operatorname{Dim}[\varphi] N_{B}-\operatorname{Dim}[\psi] N_{F}-\sum_{a}\left(d+z-\operatorname{Dim}[\varphi] \nu_{a}^{B}-\operatorname{Dim}[\psi] \nu_{a}^{F}\right),
$$

com as dimensões dos campos dadas por

$$
\operatorname{Dim}[\varphi]=\frac{d-z}{2} \quad \text { e } \quad \operatorname{Dim}[\psi]=\frac{d}{2}
$$

\subsection{Modelo de Yukawa}

Vamos discutir um modelo envolvendo campos escalar e espinorial com uma interação tipo Yukawa,

$$
\mathcal{L}_{i n t} \sim \bar{\psi} \psi \varphi
$$

De acordo com a contagem de potências acima, considerando $z=2$, o requerimento de renormalizabilidade fixa a dimensão espacial em $d=6$, ou, $D=6+1$ dimensões do espaço-tempo. As matrizes de Dirac da representação mínima são de ordem $2^{3}$. Esse é o procedimento usual para se construir representações em dimensões ímpares; usamos as matrizes da dimensão par imediatamente anterior. De uma forma geral, a ordem da matrizes é $2^{\left[\frac{D}{2}\right]}$, em que $[D / 2]$ significa o maior inteiro menor ou igual a $D / 2$.

Entretanto, não vamos usar a representação mínima das matrizes por dois motivos: primeiro, em dimensões pares do espaço-tempo, é possível construir a matriz de quiralidade $\Gamma^{5}$, a qual será definida logo abaixo. A vantagem de se ter a matriz de quiralidade é que podemos incluí-la na interação, evitando a indução de contratermos tais como $\varphi, \varphi^{3}$, devido à paridade. O segundo motivo é que a dimensão efetiva do espaço-tempo é $6+2$ $(z=2)$ e como vimos, essa é a dimensão relevante para o comportamento ultravioleta. 
Em 8 dimensões espaço-temporais, temos 8 matrizes de Dirac, $\gamma_{8}^{\mu}, \mu=0, \cdots, 7$. A matriz de quiralidade é construída a partir do produto entre as matrizes de Dirac

$$
\Gamma_{8}^{5} \equiv i \prod_{\mu=0}^{7} \gamma^{\mu},
$$

tal que $\left(\Gamma_{8}^{5}\right)^{2}=1$. Além disso, $\left\{\Gamma_{8}^{5}, \gamma_{8}^{\mu}\right\}=0$ e $\operatorname{Tr} \gamma_{8}^{\mu} \gamma_{8}^{\nu}=2^{\frac{d+2}{2}} g^{\mu \nu}$. Vamos usar essas matrizes para construir as matrizes em 7 dimensões espaço-temporais. Escolhemos 7 entre as 8 matrizes acima e a matriz $\Gamma_{7}^{5}$ pode ser escolhida como a matriz restante (a menos de um fator de $i$ ) ou como a própria matriz $\Gamma_{8}^{5}$. No que segue, omitiremos o índice inferior que indica a dimensão do espaço-tempo. Dessa forma, a lagrangiana completa com o acoplamento de Yukawa entre os campos escalar e espinorial e com $z=2$ é

$$
\begin{aligned}
\mathcal{L} & =\frac{1}{2} \partial_{0} \varphi \partial_{0} \varphi-\frac{b_{\varphi}^{2}}{2} \partial_{i} \varphi \partial_{i} \varphi-\frac{m^{2}}{2} \varphi^{2}-\frac{\alpha_{\varphi}^{2}}{2} \varphi \Delta^{2} \varphi \\
& +\bar{\psi}\left(i \gamma^{0} \partial_{0}+i b_{\psi} \gamma^{i} \partial_{i}+\alpha_{\psi} \Delta-M\right) \psi+i g \bar{\psi} \Gamma^{5} \psi \varphi-\frac{\lambda}{4 !} \varphi^{4}
\end{aligned}
$$

Note que $\left(i \gamma^{i} \partial_{i}\right)^{2}=\nabla^{2} \equiv \Delta$. Vamos considerar os parâmetros $\alpha$ 's e $b$ 's positivos. A introdução da anisotropia com $z=2$ quebra, além da simetria de Lorentz, a simetria por transformações quirais globais $\psi \rightarrow e^{i \theta \Gamma^{5}} \psi$ e $\bar{\psi} \rightarrow \bar{\psi} e^{i \theta \Gamma^{5}}$, que também é quebrada pelo termo de massa $\bar{\psi} \psi$. A interação $\varphi^{4}$ é necessária por uma questão de renormalizabilidade, devido à divergência da função de quatro pontos do campo escalar.

Tomando $z=2 \mathrm{em}(3.3)$ e renomeando os parâmetros, o propagador do campo fermiônico se reduz a

$$
S(k)=\frac{i}{\gamma^{0} k^{0}-b_{\psi} \gamma \cdot \mathbf{k}-\alpha_{\psi} \mathbf{k}^{2}-M} .
$$

As regras de Feynman para os vértices são mostradas na figura 3.1. O grau de divergência

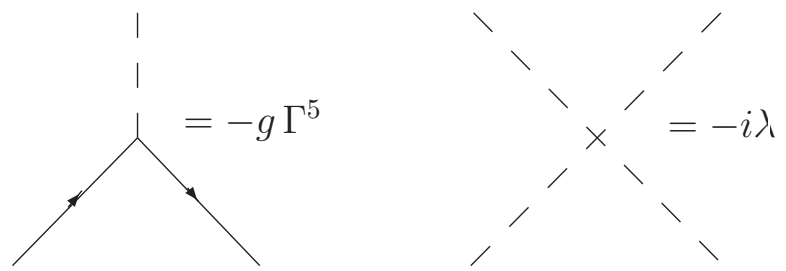

Figura 3.1: Vértices de Interação.

superficial é dado por

$$
d(G)=8-2 N_{B}-3 N_{F}
$$

e as funções de vértice divergentes são: $1 . N_{B}=2$ e $N_{F}=0, \operatorname{com} d(G)=4 ; 2 . N_{B}=4$ e $N_{F}=0, \operatorname{com} d(G)=0 ; 3 . \quad N_{B}=0$ e $N_{F}=2, \operatorname{com} d(G)=2 ; 4 . \quad N_{B}=1$ e 
$N_{F}=2$, com $d(G)=0$. Os diagramas correspondentes, relevantes para a nossa análise, são mostrados nas figuras 3.2, 3.3, 3.4 e 3.5. Assim como nos modelos envolvendo apenas campos escalares, a extração da parte divergente dos diagramas é um tanto elaborada. No presente caso, temos a complicação adicional devido aos diferentes tipos de propagadores em um mesmo diagrama. O procedimento geral é semelhante ao do capítulo anterior e está feito em detalhes no apêndice A. Na sequência, discutiremos as soluções das equações governando a evolução dos parâmetro efetivos do grupo de renormalização.

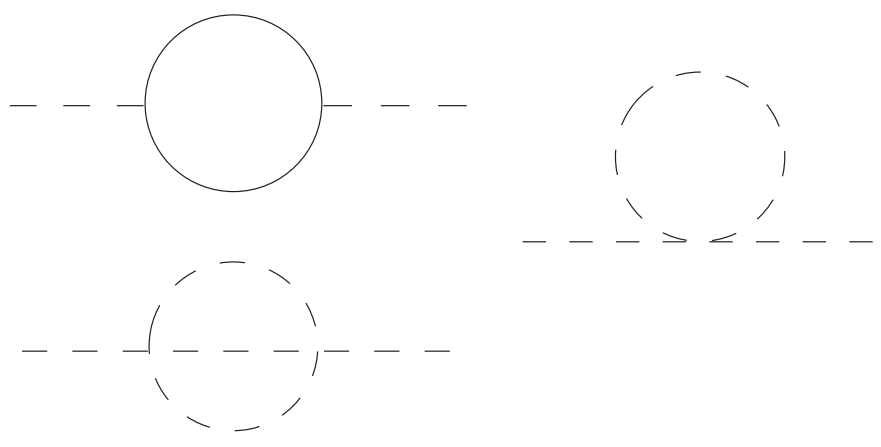

Figura 3.2: Função de dois pontos do campo escalar.

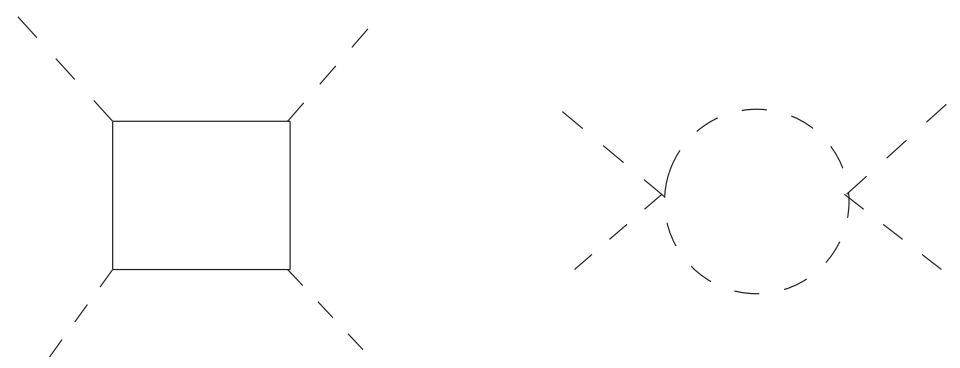

Figura 3.3: Função de quatro pontos do campo escalar.

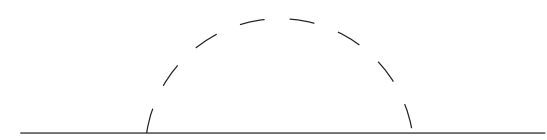

Figura 3.4: Função de dois pontos do campo espinorial. 


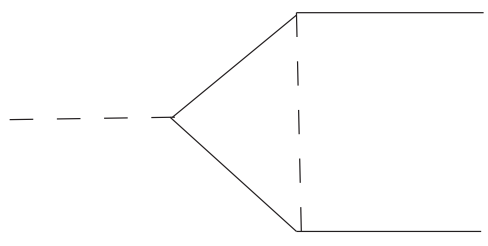

Figura 3.5: Função de três pontos.

\subsection{Parâmetros Efetivos}

Conforme mostrado no apêndice @, os resultados obtidos para os parâmetros efetivos são,

$$
\begin{gathered}
\beta_{b_{\varphi}^{2}}\left(g^{2}\right)=\frac{1}{32 \pi^{3}} \frac{\left(b_{\varphi}^{2}+2 \alpha_{\psi} M\right)}{\alpha_{\psi}^{3}} g^{2}, \\
\beta_{\alpha_{\varphi}^{2}}\left(g^{2}\right)=\frac{1}{384 \pi^{3}} \frac{\left(12 \alpha_{\varphi}^{2}+3 \alpha_{\psi}^{2}\right)}{\alpha_{\psi}^{3}} g^{2}, \\
\beta_{b_{\psi}}\left(g^{2}\right)=\frac{b_{\psi}}{192 \pi^{3}} \frac{\left(\alpha_{\varphi}+2 \alpha_{\psi}\right)}{\alpha_{\varphi} \alpha_{\psi}\left(\alpha_{\varphi}+\alpha_{\psi}\right)^{2}} g^{2}, \\
\beta_{\alpha_{\psi}}\left(g^{2}\right)=\frac{1}{192 \pi^{3}} \frac{\alpha_{\psi}\left(3 \alpha_{\varphi}+2 \alpha_{\psi}\right)}{\alpha_{\varphi}\left(\alpha_{\varphi}+\alpha_{\psi}\right)^{3}} g^{2}, \\
\beta_{g}\left(g^{3}\right)=\frac{1}{128 \pi^{3}}\left[\frac{2 \alpha_{\varphi}^{3}+4 \alpha_{\varphi}^{2} \alpha_{\psi}+3 \alpha_{\varphi} \alpha_{\psi}^{2}+2 \alpha_{\psi}^{3}}{\alpha_{\psi}^{3} \alpha_{\varphi}\left(\alpha_{\varphi}+\alpha_{\psi}\right)^{2}}\right] g^{3}
\end{gathered}
$$

$\mathrm{e}$

$$
\beta_{\lambda}=\frac{3}{512 \pi^{3}} \frac{\lambda^{2}}{\alpha_{\varphi}^{3}}-\frac{3}{8 \pi^{3}} \frac{g^{4}}{\alpha_{\psi}^{3}}+\frac{1}{16 \pi^{3}} \frac{\lambda g^{2}}{\alpha_{\psi}^{3}} .
$$

A primeira observação é que o acoplamento de Yukawa é estável no infravermelho, o que pode ser visto em (3.17), possibilitando em princípio acessar limites de baixas energias suficientemente pequenos dentro da teoria de perturbação. Os parâmetros efetivos correspondentes aos operadores com derivadas espaciais usuais $\left(b_{\varphi}\right.$ e $\left.b_{\psi}\right)$ e de ordem superior $\left(\alpha_{\varphi}\right.$ e $\left.\alpha_{\psi}\right)$, crescem com a energia. Agora nos resta encontrar a solução das equações acima para determinar o comportamento preciso dos parâmetros efetivos. Infelizmente, tais equações não se apresentam numa forma simples o suficiente para permitir soluções analíticas. Isso realmente é uma característica muito desagradável! Sendo assim, temos que partir para outra abordagem para tentar extrair alguma informação adicional.

Um procedimento natural que pode ser adotado, trata-se da identificação entre alguns dos parâmetros. Isso é interessante pois essa possibilidade pode ser associada ao fato que a simetria de Lorentz dá lugar a uma simetria anisotrópica devido à homogeneidade do 
grau de anisotropia, isto é, a deformação na lagrangiana livre dos dois campos é feita com operadores possuindo o mesmo grau de anisotropia $z=2$. A primeira tentativa de identificação corresponde à $\alpha_{\varphi} \equiv \alpha_{\psi}$. Logo percebemos que essa idetificação não é possível, pois conduz a uma inconsistência; a identificação deve ser tal que as duas equações para o parâmetro em questão se reduzam à mesma equação, no caso, $\beta_{\alpha_{\varphi}}=\beta_{\alpha_{\psi}}$, o que não ocorre com (3.14) e (3.16). Podemos tentar, no entanto, uma forma mais fraca do tipo $\alpha_{\psi} \equiv N \alpha_{\varphi}$, em que $N>0$ é um número a ser determinado tal que

$$
\beta_{\alpha_{\varphi}}=\beta_{\alpha_{\psi}}
$$

De acordo com (3.14) e (3.16), temos as duas equações diferenciais

$$
\frac{\partial \alpha_{\varphi}}{\partial t}=\frac{1}{192 \pi^{3}} f(N) \frac{g^{2}}{\alpha_{\varphi}^{2}}
$$

$\mathrm{e}$

$$
\frac{\partial \alpha_{\varphi}}{\partial t}=\frac{1}{192 \pi^{3}} l(N) \frac{g^{2}}{\alpha_{\varphi}^{2}}
$$

em que definimos as funções

$$
f(N) \equiv \frac{1}{4} \frac{\left(12+N^{2}\right)}{N^{3}}
$$

$\mathrm{e}$

$$
l(N) \equiv \frac{3+2 N}{(1+N)^{3}} .
$$

Isso mostra que não há nenhum valor de $N$ real positivo tal que $f(N)=l(N)$ e consequentemente é impossível que (3.19) seja satisfeita. Desse modo, aparentemente não temos outra escolha a não ser recorrer aos métodos numéricos.

A solução numérica permite-nos determinar todos os parâmetros efetivos. Como a equação (3.13) envolve a massa do campo fermiônico, necessitamos também da função do grupo de renormalização correspondente:

$$
\delta_{M}\left(g^{2}\right)=\frac{\left[2 \alpha_{\varphi} \alpha_{\psi}^{2} b_{\varphi}^{2}+\alpha_{\psi}^{3} b_{\varphi}^{2}+\alpha_{\varphi}^{3} b_{\psi}^{2}+2 \alpha_{\varphi}^{2} \alpha_{\psi}\left(b_{\psi}^{2}+2 \alpha_{\psi} M\right)\right]}{256 \pi^{3} \alpha_{\varphi}^{3} \alpha_{\psi}\left(\alpha_{\varphi}+\alpha_{\psi}\right)^{2}} g^{2} .
$$

As equações que governam a evolução dos parâmetros efetivos de quebra de simetria da Lorentz podem ser separados em duas partes. Na primeira, estão as equações para $\bar{\alpha}_{\varphi}$, $\bar{\alpha}_{\psi}$ e $\bar{g}$, as quais não dependem dos demais parâmetros. No segundo conjunto, estão as equações para os parâmetros $\bar{b}_{\varphi}$ e $\bar{b}_{\psi}$, que necessitam das soluções da primeira parte para serem determinados.

Antes de prosseguir, vamos ressaltar qual o significado da restauração da simetria de Lorentz nesse modelo. Como já discutimos nos estudos dos modelos escalares, não podemos tomar os parâmetros $\alpha_{\varphi}$ e $\alpha_{\psi}$ iguais a zero, pois obteríamos uma teoria nãorenormalizável. Nesse sentido, não podemos esperar uma simetria de Lorentz exata, a 
qual corresponderia $\alpha_{\varphi}=\alpha_{\psi}=0$ e $b_{\varphi}=b_{\psi}$; esses nem mesmo são pontos especiais (por exemplo, pontos fixos) das equações do grupo de renormalização. No entanto, podemos considerar a possibilidade de uma simetria de Lorentz aproximada emergindo em algum limite específico de baixas energias, dependendo de um ajuste fino entre os parâmetros envolvidos. De um modo mais preciso, podemos encontrar uma região onde os parâmetros $\bar{\alpha}_{\varphi}$ e $\bar{\alpha}_{\psi}$ são suficientemente pequenos e, além disso, os parâmetros $\bar{b}_{\varphi}$ e $\bar{b}_{\psi}$ são tais que $\bar{b}_{\varphi} \approx \bar{b}_{\psi}$. É claro, essa não é a situação ideal mas poderia fornecer ao menos uma visão positiva quanto a restauração da simetria de Lorentz em teorias anisotrópicas. Para dar uma idéia melhor sobre essa possibilidade, partímos então para uma análise numérica, como descrito na sequência.

Nosso resultados, obtidos com o auxílio do programa Mathematica, mostram que os parâmetros fermiônicos variam muito mais lentamente que os bosônicos. Realmente, as mudanças das tangentes às curvas de alguns desses parâmetro são bem pequenas produzindo a impressão de que são linhas retas. Para valores grandes de momentos, a constante de acoplamento $\bar{g}, \bar{\alpha}_{\varphi}$ e $\bar{b}_{\varphi}$ aumentam abruptamente para $t \sim 10^{9}$, indicando a existência de uma singularidade similar ao pólo de Landau encontrado em muitas teorias sem liberdade assintótica, veja as figuras 3.6, 3.7 e 3.8. Em contraste, $\bar{\alpha}_{\psi}$ e $\bar{b}_{\psi}$ aumentam lentamente como mostrado nas figuras 3.6 e 3.7. Para valores negativos de $t$, correspondendo à região de momentos pequenos, a situação geral é que todos os parâmetros diminuem e, como dito anteriormente, os parâmetros associados ao campo $\psi$ variam mais lentamente que os parâmetros associados ao campo $\varphi$ (see Figs. 3.9 and 3.10). Os gráficos foram construídos tomando como condição inicial $\bar{g}(0)=10^{-3}$ para a constante de acoplamento e os demais parâmetros todos iguais a unidade. Variações dessas condições iniciais não alteram qualitativamente o comportamento dos parâmetros efetivos. Deve ser notado que na região além do final das curvas os dados não são mais confiáveis pois o módulo de $t$ é muito grande, ao mesmo tempo que os parâmetros efetivos tornam-se muito pequenos, possibilidando então grandes erros numéricos.

Portanto, dada uma região de baixas energias, para ter uma situação com a simetria de Lorentz aproximada, deveríamos modificar as configurações inicias dos parâmetros $\alpha_{\varphi}$ e $\alpha_{\psi}$, tal que eles atinjam a faixa de valores desejados na região de energia especificada. Por exemplo, como os $\bar{\alpha}$ 's decrescem monotonicamente quando $t$ diminui, isso pode ser facilmente obtido escolhendo os valores iniciais de $\alpha_{\varphi}$ e $\alpha_{\psi}$ iguais ao máximo que eles deveriam ter na região de interesse. Além disso, precisamos ajustar as configurações iniciais de $b_{\varphi}$ e $b_{\psi}$, tal que $\bar{b}_{\varphi} \approx \bar{b}_{\psi}$ na mesma região. Note que, esses dois requerimentos podem ser satisfeitos simultaneamente devido ao desacoplamento das equações para $\bar{\alpha}$ 's e $\bar{b}$ 's. Como uma lição final, o estudo do modelo de Yukawa nos dá uma boa idéia que a questão da restauração da simetria de Lorentz torna-se muito delicada e dependendo de muitos ajustes entre os parâmetros no caso de teorias anisotrópicas envolvendo mais que um tipo de campo. Essa situação fica mais complexa a medida que o número de campos é aumentado. 

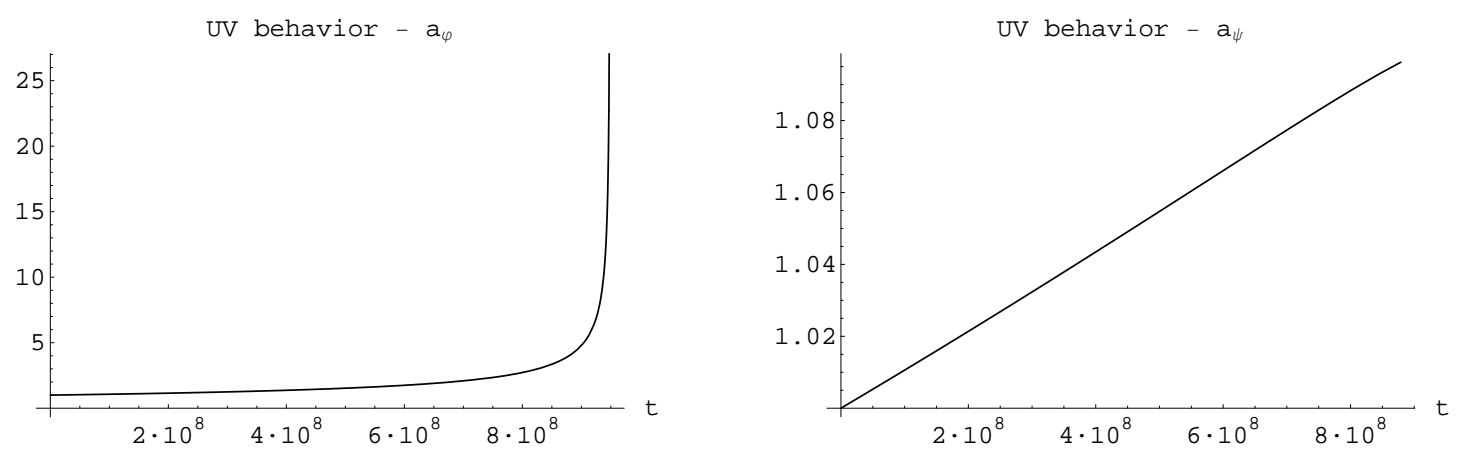

Figura 3.6: Comportamento ultravioleta dos parâmetros $a_{\varphi}$ e $a_{\psi}$.
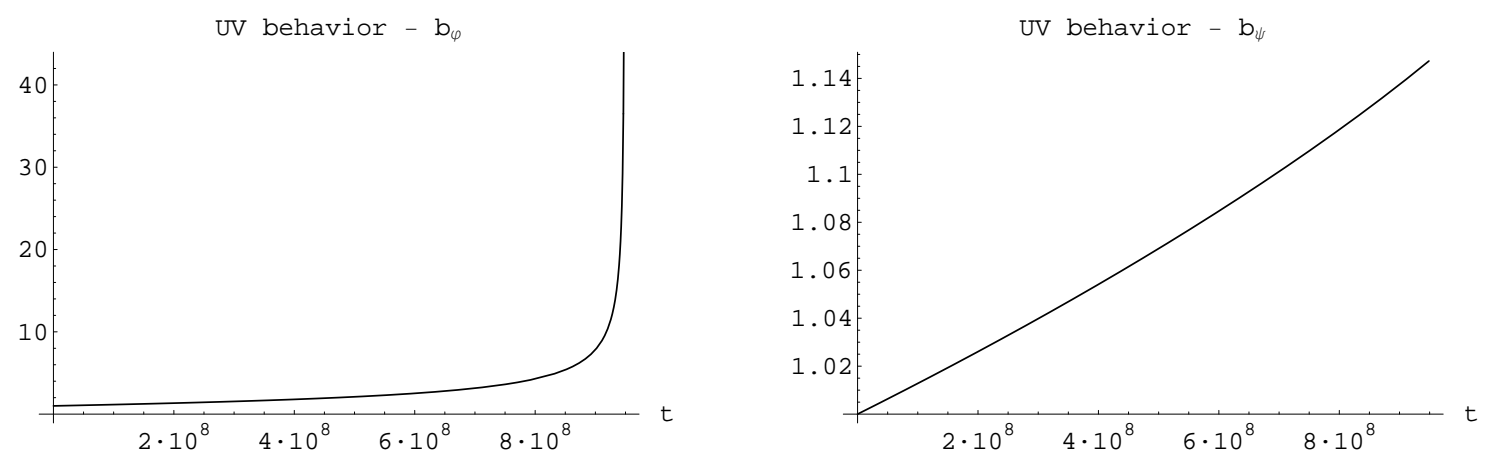

Figura 3.7: Comportamento ultravioleta dos parâmetros $b_{\varphi}$ e $b_{\psi}$.
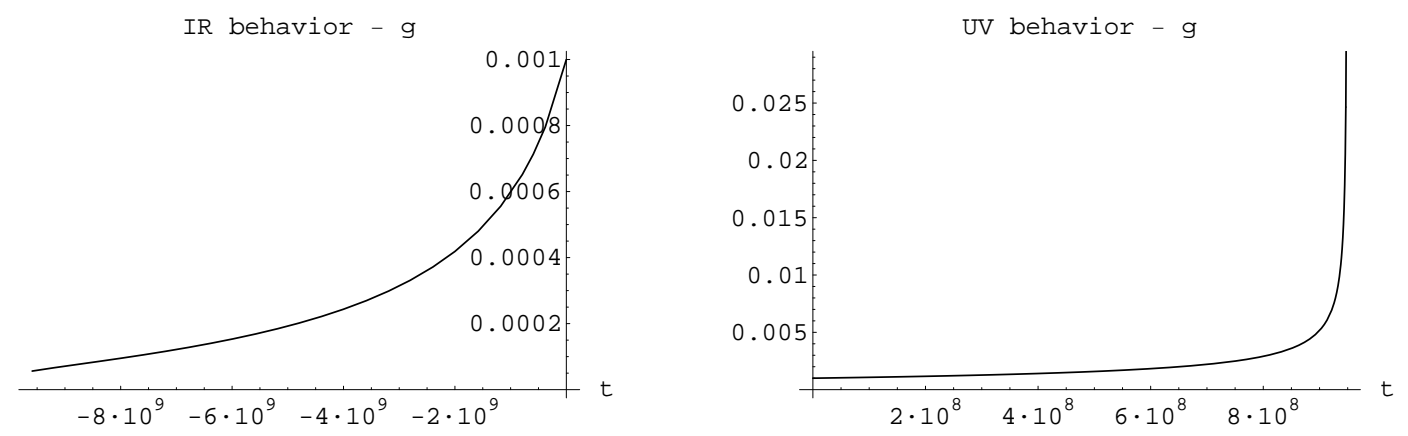

Figura 3.8: Comportamento geral da constante de acoplamento $g$. A singularidade na região UV é similar a um pólo de Landau. 

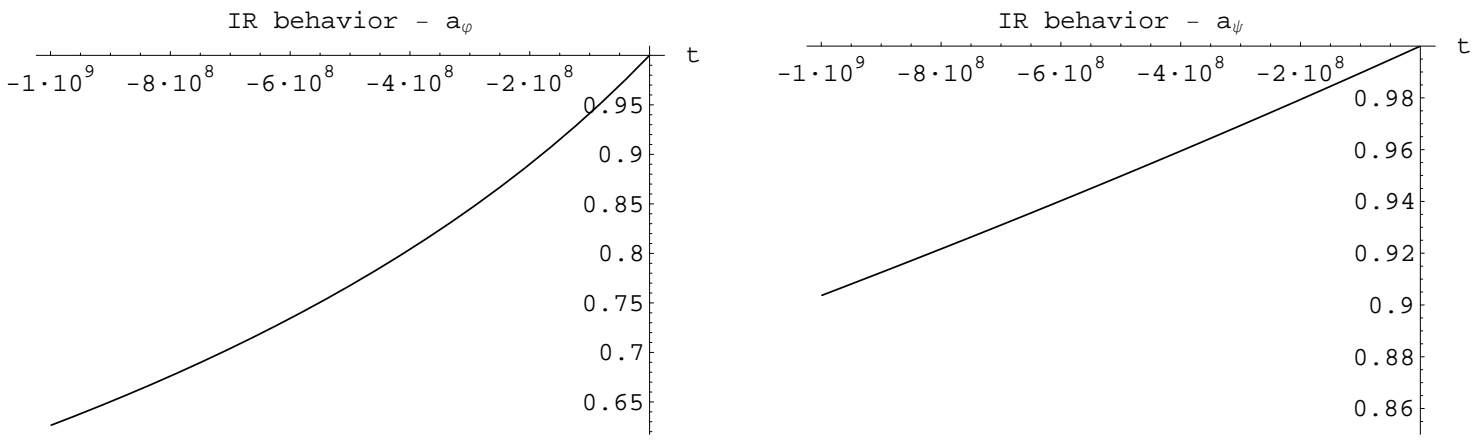

Figura 3.9: Comportamento infravermelho dos parâmetros $a_{\varphi}$ e $a_{\psi}$.
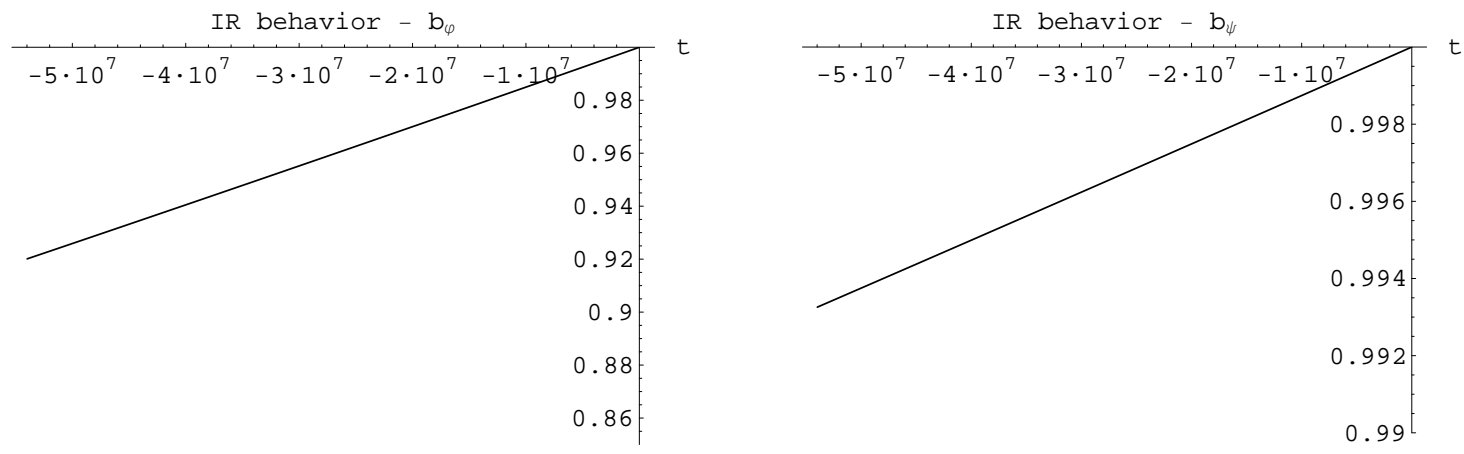

Figura 3.10: Comportamento infravermelho dos parâmetros $b_{\varphi}$ e $b_{\psi}$. 


\section{Capítulo 4}

\section{Identidades de Ward}

Neste capítulo, discutiremos brevemente alguns aspectos clássicos e quânticos de simetrias em teorias relativísticas. A construção de correntes quânticas introduz divergências adicionais que necessitam ser regularizadas, o que pode ser feito de uma maneira sistemática usando o método dos produtos normais. Em seguida, generalizaremos esse método para teorias anisotrópicas e concluiremos com algumas aplicações [47].

\subsection{Simetrias Clássicas}

Uma maneira muito eficiente e elegante de se estudar um sistema físico consiste na análise das simetrias subjacentes. A existência de simetrias contínuas implica na existência de quantidades conservadas. Esse é o conteúdo do teorema de Noether, o qual passamos a descrever.

Queremos construir a corrente de Noether associada a uma transformação contínua arbitrária do campo $\varphi \rightarrow \varphi+\delta \varphi$. Se essa transformação for uma simetria, a corrente será conservada. Para isso, vamos considerar uma teoria com derivadas de ordem superior, que será útil também no estudo de teorias anisotrópicas. Para um campo escalar real, vamos supor que a lagrangiana dependa do campo e de suas primeiras e segundas derivada: $1: \mathcal{L}\left(\varphi, \partial_{\mu} \varphi, \partial_{\mu} \partial_{\nu} \varphi\right)$. A discussão a seguir pode ser facilmente generalizada para teorias com outros tipos de campos (espinoriais, de calibre, etc). A extremização da ação correspondente conduz à equação de movimento

$$
-\frac{\partial \mathcal{L}}{\partial \varphi}+\partial_{\mu} \frac{\partial \mathcal{L}}{\partial \partial_{\mu} \varphi}-\partial_{\mu} \partial_{\nu} \frac{\partial \mathcal{L}}{\partial \partial_{\mu} \partial_{\nu} \varphi}=0
$$

Sob uma variação contínua do campo $\varphi \rightarrow \varphi+\delta \varphi$, a densidade de lagrangiana $\mathcal{L}$ muda

\footnotetext{
${ }^{1}$ A generalização para teorias com derivadas de ordens mais altas é feita no apêndice B.
} 
como

$$
\delta \mathcal{L}=\frac{\partial \mathcal{L}}{\partial \varphi}+\frac{\partial \mathcal{L}}{\partial \partial_{\mu} \varphi} \delta \partial_{\mu} \varphi+\frac{\partial \mathcal{L}}{\partial \partial_{\mu} \partial_{\nu} \varphi} \delta \partial_{\mu} \partial_{\nu} \varphi
$$

Usando a equação de movimento, a expressão acima pode ser escrita como

$$
\delta \mathcal{L}=\partial_{\mu}\left[\frac{\partial \mathcal{L}}{\partial \partial_{\mu} \varphi} \delta \varphi+\frac{\partial \mathcal{L}}{\partial \partial_{\mu} \partial_{\nu} \varphi} \stackrel{\leftrightarrow}{\partial} \delta \varphi\right]
$$

em que $A \stackrel{\leftrightarrow}{\partial_{\mu}} B \equiv A \partial_{\mu} B-\left(\partial_{\mu} A\right) B$. Por outro lado, no caso mais geral, a variação da lagrangiana deve ter a forma $\delta \mathcal{L}=\partial_{\mu} S^{\mu}+\mathcal{O}$, em que $\mathcal{O}$ representa genericamente um termo de quebra de simetria; se $\mathcal{O}=0$, a transformação é uma simetria da teoria. Desse modo, podemos definir a corrente

$$
J^{\mu} \equiv \frac{\partial \mathcal{L}}{\partial \partial_{\mu} \varphi} \delta \varphi+\frac{\partial \mathcal{L}}{\partial \partial_{\mu} \partial_{\nu} \varphi} \overleftrightarrow{\partial}_{\nu} \delta \varphi-S^{\mu}
$$

tal que

$$
\partial_{\mu} J^{\mu}=\mathcal{O}
$$

Portanto, quando $\mathcal{O}=0$, teremos a carga conservada

$$
Q \equiv \int d^{D-1} x J^{0}(x)
$$

Uma questão muito natural e importante trata-se da análise das simetrias no caso quântico. Em outras palavras, se as simetrias da teoria clássica sobrevivem ao processo de quantização. Devido ao procedimento de renormalização isso não é claro e será discutido a seguir.

\subsection{Simetrias Quânticas}

De uma maneira geral, na situação quântica, objetos clássicos são promovidos a operadores e as equações clássicas passam a ser válidas como valores esperados:

$$
\text { Equação Clássica } \Rightarrow\langle\text { Equação Operatorial }\rangle \text {. }
$$

Esse resultado é conhecido como teorema de Ehrenfest. No contexto da teoria quântica de campos, os objetos básicos são as funções de Green, dadas pelo valor esperado no vácuo de um produto de operadores de campo ordenados temporalmente:

$$
\left\langle 0\left|T \varphi\left(x_{1}\right) \cdots \varphi\left(x_{N}\right)\right| 0\right\rangle .
$$


Uma simetria da teoria quântica deve ser refletida na invariância das funções de Green:

$$
0=\delta\left\langle 0\left|T \varphi\left(x_{1}\right) \cdots \varphi\left(x_{N}\right)\right| 0\right\rangle=\sum_{j}\left\langle 0\left|T \varphi\left(x_{1}\right) \cdots \delta \varphi\left(x_{j}\right) \cdots \varphi\left(x_{N}\right)\right| 0\right\rangle .
$$

De uma maneira heurística, podemos pensar que o teorema de Ehrenfest se traduza para a teoria de campos como relações operatorias inseridas em funções de Green. Em particular, para o caso da corrente de Noether, a versão quântica de (4.5) tem a forma

$$
\partial_{\mu}\left\langle 0\left|T J^{\mu}(x) X\right| 0\right\rangle=\langle 0|T \mathcal{O}(x) X| 0\rangle-i \sum_{k} \delta\left(x-x_{k}\right)\left\langle 0\left|T \delta \varphi\left(x_{k}\right) X_{k}\right| 0\right\rangle,
$$

com $X \equiv \prod_{j} \varphi\left(x_{j}\right)$ e $X_{k}$ igual à $X$ porém com a omissão do campo referente à coordenada $x_{k}$. Observe que o último termo reproduz corretamente a condição de simetria das funções de Green (4.9) com $\mathcal{O}=0$, após uma integração sobre o espaço-tempo (na ausência de forças de longo alcance). Relações do tipo (4.10) são conhecias como identidades de Ward e estabelecem relações entre as funções de Green. As identidades de Ward tem um papel fundamental em diversos aspectos da teoria de campos [45, 48.

A relação (4.10) é uma expressão formal que ainda necessita de uma prescrição para ser feita precisa. Isso porque os termos $J^{\mu}(x)$ e $\mathcal{O}(x)$ envolvem, em geral, produtos de campos no mesmo ponto, os chamados operadores compostos. Esses operadores compostos trazem novas divergências que devem ser eliminadas. Um exemplo simples é proporcionado pela função de dois pontos de um campo livre em quatro dimensões espaço-temporais:

$$
\langle 0|T \varphi(x) \varphi(y)| 0\rangle \sim \frac{1}{(x-y)^{2}}, \text { quando } y \rightarrow x .
$$

Isso significa que as correntes devem ser regularizadas. Imediatamente, vemos que na teoria quântica temos classes de correntes, diferindo por partes finitas. Um guia para se regularizar uma corrente consiste em empregar o mesmo esquema utilizado para regularizar e então renormalizar a teoria. Usualmente, a escolha do esquema está relacionada com a preservação das simetrias envolvidas. Por exemplo, numa teoria regularizada dimensionalmente, a subtração de pólos é indicada para a regularização da corrente. Naturalmente, a regularização apropriada é aquela que respeita as simetrias ao nível quântico. Quando não é possível escolher um esquema que preserve as simetrias, a teoria é dita conter anomalias. Em outras palavras, a anomalia pode ser entendida como uma simetria clássica que é quebrada por correções radiativas. As anomalias têm consequências importantes tanto no sentido de consistência dos cálculos perturbativos bem como em aplicações fenomenológicas [49]. Um exemplo bem conhecido é a anomalia da corrente axial na eletrodinâmica quântica:

$$
\partial_{\mu} J_{5}^{\mu}=2 i M \bar{\psi} \gamma^{5} \psi+\frac{e^{2}}{16 \pi^{2}} F_{\mu \nu} \widetilde{F}^{\mu \nu},
$$


sendo que a divergência da corrente não se anula quando $M \rightarrow 0$, devido ao termo proporcional a $F_{\mu \nu} \widetilde{F}^{\mu \nu}$.

Uma maneira sistemática para tratar os problemas mencionados e determinar as identidades de Ward e possíveis anomalias, deve-se ao método dos produtos normais [50, 51]. Esse método está ligado ao esquema de renormalização BPHZ e é baseado na subtração de divergências mediante aplicação de operadores de Taylor. Discutiremos isso em detalhes na próxima seção, mas para a presente discussão é suficiente definir um produto normal de grau $\delta, N_{\delta}$, associado a um operador composto $\mathcal{O}$, com $\delta \geq \operatorname{Dim}[\mathcal{O}]$, tal que uma função de Green com a inserção de $N_{\delta}[\mathcal{O}]$,

$$
\left\langle 0\left|T N_{\delta}[\mathcal{O}] \varphi\left(x_{1}\right) \cdots \varphi\left(x_{N}\right)\right| 0\right\rangle
$$

é bem definida. Assim, na teoria quântica os operadores compostos devem ser definidos com um produto normal associado. Esses produtos normais satisfazem um conjunto de regras que permitem a derivação das identidades de Ward. Nesse contexto, a forma bem definida da expressão (4.10) torna-se

$$
\partial_{\mu}\left\langle 0\left|T N_{\delta-1}\left[J^{\mu}\right](x) X\right| 0\right\rangle=\left\langle 0\left|T N_{\delta}[\mathcal{O}](x) X\right| 0\right\rangle-i \sum_{k} \delta\left(x-x_{k}\right)\left\langle 0\left|T \delta \varphi\left(x_{k}\right) X_{k}\right| 0\right\rangle .
$$

A origem das anomalias nessa expressão é devido aos termos com subtrações a mais que o necessário contidas em $N_{\delta}[\mathcal{O}]$ para tornar a inserção do operador composto finita. Por exemplo, o termo de quebra no caso da corrente axial é da forma $M\left\langle 0\left|T N_{4}\left[\bar{\psi} \gamma^{5} \psi\right](x) X\right| 0\right\rangle$. Entretanto, a dimensão do operador $\bar{\psi} \gamma^{5} \psi$ é três, conduzindo a uma subtração adicional. Os termos provenientes dessa subtração dão origem à anomalia em (4.12).

\subsection{Identidades de Ward em Teorias com Anisotropia}

Agora, vamos discutir a generalização do método dos produtos normais para teorias com escalonamento anisotrópico entre o espaço e o tempo.

\subsubsection{Produtos Normais}

Nos estudos deste capítulo, vamos considerar como protótipo o modelo escalar $\varphi^{4}$ renormalizado via o método $\mathrm{BPHZ}, \operatorname{com} z=2$ e em $d=6$ dimensões espaciais, dado por

$$
\mathcal{L}=\frac{1}{2}(1+A) \partial_{0} \varphi \partial_{0} \varphi-\frac{\left(b^{2}+B\right)}{2} \partial_{i} \varphi \partial_{i} \varphi-\frac{\left(a^{2}+C\right)}{2} \Delta \varphi \Delta \varphi-\frac{\left(m^{2}+D\right)}{2} \varphi^{2}-\frac{(\lambda+E)}{4 !} \varphi^{4}
$$

em que $A, B, C, D$ e $E$ são contra-termos finitos determinados por meio das condições de normalização, $b^{2}, a^{2}, m^{2}$ e $\lambda$ são os parâmetros originais da teoria. Apesar de nos restringir 
a campos escalares, a análise a seguir pode ser facilmente generalizada para outros tipos de campos. Além disso, sempre que oportuno vamos estender nossa discussão para valores arbitrários de $z$.

Seja $\mathcal{O}$ algum produto formal de campos e suas derivadas no mesmo ponto. Associado ao operador $\mathcal{O}$, existe um conjunto infinito de produtos normais $N_{\delta}$, em que $\delta$ é o grau do produto normal e deve ser um inteiro maior ou igual à dimensão canônica do operador $\mathcal{O} ; \delta \geq \operatorname{Dim}[\mathcal{O}]$. Assim, o produto normal é definido como

$$
\left\langle 0\left|T N_{\delta}[\mathcal{O}] X\right| 0\right\rangle \equiv \text { Parte Finita de }\langle 0|T \mathcal{O} X| 0\rangle,
$$

com $X \equiv \prod_{i} \varphi\left(x_{i}\right)$. A prescrição para a parte finita pode ser obtida como segue. Na ausência do produto formal, as funções de Green já estão normalizadas via o esquema BPHZ. Por outro lado, na presença dos produtos formais, temos divergências adicionais que necessitam ser subtraídas. De fato, o grau de divergência superficial no caso anisotrópico para funções de Green sem inserção de produtos normais é

$$
d(G)=d+z-\operatorname{Dim}[\varphi] N_{B}-\operatorname{Dim}[\psi] N_{F}-\sum_{a}\left(d+z-\operatorname{Dim}\left[V_{a}\right]\right)
$$

em que,

$$
\operatorname{Dim}[\varphi]=\frac{d-z}{2} \quad \text { e } \quad \operatorname{Dim}[\psi]=\frac{d}{2}
$$

e $\operatorname{Dim}\left[V_{a}\right]=D_{a}+\operatorname{Dim}[\varphi] \nu_{a}^{B}+\operatorname{Dim}[\psi] \nu_{a}^{F} . \quad \nu_{a}^{B}$ e $\nu_{a}^{F}$ são os números de linhas bosônicas e fermiônicas juntando-se no vértice $V_{a}$ e $D_{a}$ refere-se aos fatores de momento nos vértices, contando-se $z$ para componentes tipo tempo e um para componentes tipo espaço. Essa fórmula generaliza aquelas obtidas em (2.7) e (3.6).

$\mathrm{Na}$ presença do produto normal, temos

$$
d(G)=d+z-\operatorname{Dim}[\varphi] N_{B}-\operatorname{Dim}[\psi] N_{F}-\sum_{a}\left(d+z-\operatorname{Dim}\left[V_{a}\right]\right)-(d+z-\operatorname{Dim}[\mathcal{O}])
$$

Para teorias renormalizáveis, temos $d+z-\operatorname{Dim}\left[V_{a}\right]=0$, tal que

$$
d(G)=\operatorname{Dim}[\mathcal{O}]-\operatorname{Dim}[\varphi] N_{B}-\operatorname{Dim}[\psi] N_{F} .
$$

Substituindo $\operatorname{Dim}[\mathcal{O}] \rightarrow \delta$, lembrando que $\delta \geq \operatorname{Dim}[\mathcal{O}]$, temos

$$
\delta(\gamma)=\delta-\operatorname{Dim}[\varphi] N_{B}-\operatorname{Dim}[\psi] N_{F}
$$

Claramente, uma subtração com um operador de Taylor da ordem $\delta(\gamma)$ produzirá um resultado finito. Além disso, para valores $\delta>\operatorname{Dim}[\mathcal{O}]$, teremos subtrações adicionais. Entretanto, essas subtrações adicionais são finitas no ultravioleta. Finalmente, vamos definir a prescrição para um diagrama arbitrário: empregamos o operador de Taylor de grau $\delta(\gamma)$, arranjado de acordo com a fórmula da floresta:

$$
\delta(\gamma)=\left\{\begin{array}{cl}
\delta-\operatorname{Dim}[\varphi] N_{B}-\operatorname{Dim}[\psi] N_{F}, & \text { se } V_{\mathcal{O}} \in \gamma \\
d+z-\operatorname{Dim}[\varphi] N_{B}-\operatorname{Dim}[\psi] N_{F}, & \text { se } V_{\mathcal{O}} \notin \gamma
\end{array}\right.
$$


sendo $N_{B}$ e $N_{F}$ as linhas externas ao diagrama $\gamma$. Os produtos normais definidos dessa forma satisfazem um conjunto de regras que nos possibilita realizar um estudo sistemático das identidades de Ward e de suas anomalias.

\section{Regra de Derivação}

As regras de derivação permitem comutar derivadas com os produtos normais, levando em conta uma mudança no grau do produto em questão. Podemos obter sem dificuldades as relações

$$
\partial_{0}\left\langle 0\left|T N_{\delta}[\mathcal{O}] X\right| 0\right\rangle=\left\langle 0\left|T N_{\delta+z}\left[\partial_{0} \mathcal{O}\right] X\right| 0\right\rangle
$$

e

$$
\partial_{i}\left\langle 0\left|T N_{\delta}[\mathcal{O}] X\right| 0\right\rangle=\left\langle 0\left|T N_{\delta+1}\left[\partial_{i} \mathcal{O}\right] X\right| 0\right\rangle .
$$

Esses resultados podem ser obtidos a partir da observação que o operador de Taylor anisotrópico satisfaz: $p^{0} t_{p}^{\delta} f(p)=t_{p}^{\delta+z}\left(p^{0} f(p)\right)$ e $p^{i} t_{p}^{\delta} f(p)=t_{p}^{\delta+1}\left(p^{i} f(p)\right)$.

\section{Equação de Movimento}

Trata-se da versão quântica da equação de movimento e para o modelo (4.15) é dada por

$$
\begin{aligned}
& \left\langle 0\left|\mathrm{~T} N_{8}\left[\varphi\left(\partial_{0}^{2}-b^{2} \Delta+a^{2} \Delta^{2}+m^{2}\right) \varphi\right](x) X\right| 0\right\rangle=-A\left\langle 0\left|T N_{8}\left[\varphi \partial_{0}^{2} \varphi\right] X\right| 0\right\rangle \\
+ & B\left\langle 0\left|T N_{8}[\varphi \Delta \varphi] X\right| 0\right\rangle-C\left\langle 0\left|T N_{8}\left[\varphi \Delta^{2} \varphi\right] X\right| 0\right\rangle-D\left\langle 0\left|T N_{8}\left[\varphi^{2}\right] X\right| 0\right\rangle \\
- & \frac{(\lambda+E)}{3 !}\left\langle 0\left|\mathrm{~T} N_{8}\left[\varphi^{4}\right](x) X\right| 0\right\rangle-i \sum_{i=1}^{N} \delta\left(x-x_{i}\right)\langle 0|\mathrm{~T} X| 0\rangle,
\end{aligned}
$$

em que $X=\prod_{i} \varphi\left(x_{i}\right)$. Essa equação pode ser deduzida notando que o operador do lado esquerdo atuando sobre $\varphi$, escrito no espaço de momentos, é igual a $-i$ vezes o inverso do propagador livre.

\section{Identidades de Zimmermann}

As identidades de Zimmermann são relações entre produtos normais de graus diferentes associados ao mesmo produto formal. Vamos discutir alguns exemplos. Primeiro, para o operador $\mathcal{O}=\varphi^{2}$, com dimensão $\operatorname{Dim}\left[\varphi^{2}\right]=4$, queremos obter os termos coletivamente denotados por $\mathcal{R}_{1}$ na relação abaixo

$$
\left\langle 0\left|T N_{8}\left[\varphi^{2}\right] X\right| 0\right\rangle=\left\langle 0\left|T N_{4}\left[\varphi^{2}\right] X\right| 0\right\rangle+\mathcal{R}_{1} .
$$

As duas prescrições são: $\delta_{1}(\gamma)=4-2 N_{\gamma}$ para $N_{4}$ e $\delta_{2}(\gamma)=8-2 N_{\gamma}$ para $N_{8}$. 
a. $N_{\gamma}=2, \delta_{1}(\gamma)=0$ e $\delta_{2}(\gamma)=4$. O termo sem derivadas está presente nas duas subtrações e assim não contribui para a diferença $\mathcal{R}_{1}$. A subtração com $\delta_{2}$ conduz aos seguintes termos: $\left\langle 0\left|T N_{8}[\varphi \Delta \varphi] X\right| 0\right\rangle$ e $\left\langle 0\left|T N_{8}\left[\partial_{i} \varphi \partial_{i} \varphi\right] X\right| 0\right\rangle$ de segunda ordem nas derivadas, e $\left\langle 0\left|T N_{8}\left[\varphi \partial_{0}^{2} \varphi\right] X\right| 0\right\rangle,\left\langle 0\left|T N_{8}\left[\partial_{0} \varphi \partial_{0} \varphi\right] X\right| 0\right\rangle,\left\langle 0\left|T N_{8}[\Delta \varphi \Delta \varphi] X\right| 0\right\rangle,\left\langle 0\left|T N_{8}\left[\varphi \Delta^{2} \varphi\right] X\right| 0\right\rangle$, $\left\langle 0\left|T N_{8}\left[\partial_{i} \partial_{j} \varphi \partial_{i} \partial_{j} \varphi\right] X\right| 0\right\rangle$ e $\left\langle 0\left|T N_{8}\left[\Delta \partial_{i} \varphi \partial_{i} \varphi\right] X\right| 0\right\rangle$ de quarta ordem nas derivadas. Note que os termos de segunda ordem ainda podem ser reduzidos.

b. $N_{\gamma}=4, \delta_{1}(\gamma)=-4$ e $\delta_{2}(\gamma)=0$. Temos apenas o termo sem derivadas correspondendo à subtração de acordo com $\delta_{2}$. Assim, o contra-termo é $\left\langle 0\left|T N_{8}\left[\varphi^{4}\right] X\right| 0\right\rangle$.

Reunindo todas as possibilidades, obtemos

$$
\begin{aligned}
& \left\langle 0\left|T N_{8}\left[\varphi^{2}\right] X\right| 0\right\rangle=\left\langle 0\left|T N_{4}\left[\varphi^{2}\right] X\right| 0\right\rangle+p_{1}\left\langle 0\left|T N_{8}[\varphi \Delta \varphi] X\right| 0\right\rangle \\
+ & p_{2}\left\langle 0\left|T N_{8}\left[\partial_{i} \varphi \partial_{i} \varphi\right] X\right| 0\right\rangle+q_{1}\left\langle 0\left|T N_{8}\left[\varphi \partial_{0}^{2} \varphi\right] X\right| 0\right\rangle+q_{2}\left\langle 0\left|T N_{8}\left[\partial_{0} \varphi \partial_{0} \varphi\right] X\right| 0\right\rangle \\
+ & q_{3}\left\langle 0\left|T N_{8}[\Delta \varphi \Delta \varphi] X\right| 0\right\rangle+q_{4}\left\langle 0\left|T N_{8}\left[\varphi \Delta^{2} \varphi\right] X\right| 0\right\rangle+q_{5}\left\langle 0\left|T N_{8}\left[\partial_{i} \partial_{j} \varphi \partial_{i} \partial_{j} \varphi\right] X\right| 0\right\rangle \\
+ & q_{6}\left\langle 0\left|T N_{8}\left[\Delta \partial_{i} \varphi \partial_{i} \varphi\right] X\right| 0\right\rangle+q_{7}\left\langle 0\left|T N_{8}\left[\varphi^{4}\right] X\right| 0\right\rangle .
\end{aligned}
$$

Os fatores $p_{i}, q_{j}$ são determinados a partir das condições de normalização, as quais serão discutidas em breve. No momento, uma simples análise dimensional mostra que: $\operatorname{Dim}\left[p_{i}\right]=$ -2 e $\operatorname{Dim}\left[q_{j}\right]=-4$. Note que os termos $\left\langle 0\left|T N_{8}[\varphi \Delta \varphi] X\right| 0\right\rangle$ e $\left\langle 0\left|T N_{8}\left[\partial_{i} \varphi \partial_{i} \varphi\right] X\right| 0\right\rangle$ contêm subtrações adicionais e ainda podem ser reduzidos. Consideremos então o operador $\mathcal{O}=\varphi \Delta \varphi$, com dimensão $\operatorname{Dim}[\varphi \Delta \varphi]=6$ :

$$
\left\langle 0\left|T N_{8}[\varphi \Delta \varphi] X\right| 0\right\rangle=\left\langle 0\left|T N_{6}[\varphi \Delta \varphi] X\right| 0\right\rangle+\mathcal{R}_{2} .
$$

As duas prescrições são: $\delta_{1}(\gamma)=6-2 N_{\gamma}$ e $\delta_{2}(\gamma)=8-2 N_{\gamma}$.

a. $N_{\gamma}=2, \delta_{1}(\gamma)=2$ e $\delta_{2}(\gamma)=4$. Os termos de ordem zero e de segunda ordem estão presentes nas duas subtrações e cancelam-se entre si. Assim, temos apenas os termos de quarta ordem vindo de $\delta_{2}:\left\langle 0\left|T N_{8}\left[\varphi \partial_{0}^{2} \varphi\right] X\right| 0\right\rangle,\left\langle 0\left|T N_{8}\left[\partial_{0} \varphi \partial_{0} \varphi\right] X\right| 0\right\rangle,\left\langle 0\left|T N_{8}[\Delta \varphi \Delta \varphi] X\right| 0\right\rangle$ e $\left\langle 0\left|T N_{8}\left[\varphi \Delta^{2} \varphi\right] X\right| 0\right\rangle$.

b. $\quad N_{\gamma}=4, \delta_{1}(\gamma)=-2$ e $\delta_{2}(\gamma)=0$. Temos apenas o termo de ordem zero $\left\langle 0\left|T N_{8}\left[\varphi^{4}\right] X\right| 0\right\rangle$.

Considerando todos os termos, obtemos

$$
\begin{array}{ll} 
& \left\langle 0\left|T N_{8}[\varphi \Delta \varphi] X\right| 0\right\rangle=\left\langle 0\left|T N_{6}[\varphi \Delta \varphi] X\right| 0\right\rangle+f_{1}\left\langle 0\left|T N_{8}\left[\varphi \partial_{0}^{2} \varphi\right] X\right| 0\right\rangle \\
+ & f_{2}\left\langle 0\left|T N_{8}\left[\partial_{0} \varphi \partial_{0} \varphi\right] X\right| 0\right\rangle+f_{3}\left\langle 0\left|T N_{8}[\Delta \varphi \Delta \varphi] X\right| 0\right\rangle+f_{4}\left\langle 0\left|T N_{8}\left[\varphi \Delta^{2} \varphi\right] X\right| 0\right\rangle \\
+\quad & +f_{5}\left\langle 0\left|T N_{8}\left[\partial_{i} \partial_{j} \varphi \partial_{i} \partial_{j} \varphi\right] X\right| 0\right\rangle+f_{6}\left\langle 0\left|T N_{8}\left[\Delta \partial_{i} \varphi \partial_{i} \varphi\right] X\right| 0\right\rangle+f_{7}\left\langle 0\left|T N_{8}\left[\varphi^{4}\right] X\right| 0\right\rangle .
\end{array}
$$

Como antes, fazendo uma análise dimensional, vemos que $\operatorname{Dim}\left[f_{i}\right]=-2$. O operador $\mathcal{O}=\partial_{i} \varphi \partial_{i} \varphi$ conduz aos mesmos tipos de contribuições:

$$
\begin{array}{ll} 
& \left\langle 0\left|T N_{8}\left[\partial_{i} \varphi \partial_{i} \varphi\right] X\right| 0\right\rangle=\left\langle 0\left|T N_{6}\left[\partial_{i} \varphi \partial_{i} \varphi\right] X\right| 0\right\rangle+f_{1}^{\prime}\left\langle 0\left|T N_{8}\left[\varphi \partial_{0}^{2} \varphi\right] X\right| 0\right\rangle \\
+ & f_{2}^{\prime}\left\langle 0\left|T N_{8}\left[\partial_{0} \varphi \partial_{0} \varphi\right] X\right| 0\right\rangle+f_{3}^{\prime}\left\langle 0\left|T N_{8}[\Delta \varphi \Delta \varphi] X\right| 0\right\rangle+f_{4}^{\prime}\left\langle 0\left|T N_{8}\left[\varphi \Delta^{2} \varphi\right] X\right| 0\right\rangle \\
+\quad & +f_{5}^{\prime}\left\langle 0\left|T N_{8}\left[\partial_{i} \partial_{j} \varphi \partial_{i} \partial_{j} \varphi\right] X\right| 0\right\rangle+f_{6}^{\prime}\left\langle 0\left|T N_{8}\left[\Delta \partial_{i} \varphi \partial_{i} \varphi\right] X\right| 0\right\rangle+f_{7}^{\prime}\left\langle 0\left|T N_{8}\left[\varphi^{4}\right] X\right| 0\right\rangle .
\end{array}
$$


Claramente, quando integrados sobre o espaço-tempo, podemos relacionar produtos normais diferindo por termos de superfície, por exemplo, $\int d x^{0} d^{6} x\left\langle 0\left|T N_{8}[\varphi \Delta \varphi] X\right| 0\right\rangle=$ $-\int d x^{0} d^{6} x\left\langle 0\left|T N_{8}\left[\partial_{i} \varphi \partial_{i} \varphi\right] X\right| 0\right\rangle$. Dessa forma, alguns termos do lado direito podem ser deixados iguais depois de integrações por partes.

Com essas relações, podemos obter a forma completamente reduzida do produto normal em (4.27):

$$
\begin{aligned}
& \left\langle 0\left|T N_{8}\left[\varphi^{2}\right] X\right| 0\right\rangle=\left\langle 0\left|T N_{4}\left[\varphi^{2}\right] X\right| 0\right\rangle+p_{1}\left\langle 0\left|T N_{6}[\varphi \Delta \varphi] X\right| 0\right\rangle+p_{2}\left\langle 0\left|T N_{6}\left[\partial_{i} \varphi \partial_{i} \varphi\right] X\right| 0\right\rangle \\
+ & \left(q_{1}+p_{1} f_{1}+p_{2} f_{1}^{\prime}\right)\left\langle 0\left|T N_{8}\left[\varphi \partial_{0}^{2} \varphi\right] X\right| 0\right\rangle+\left(q_{2}+p_{1} f_{2}+p_{2} f_{2}^{\prime}\right)\left\langle 0\left|T N_{8}\left[\partial_{0} \varphi \partial_{0} \varphi\right] X\right| 0\right\rangle \\
+ & \left(q_{3}+p_{1} f_{3}+p_{2} f_{3}^{\prime}\right)\left\langle 0\left|T N_{8}[\Delta \varphi \Delta \varphi] X\right| 0\right\rangle+\left(q_{4}+p_{1} f_{4}+p_{2} f_{4}^{\prime}\right)\left\langle 0\left|T N_{8}\left[\varphi \Delta^{2} \varphi\right] X\right| 0\right\rangle \\
+ & \left(q_{5}+p_{1} f_{5}+p_{2} f_{5}^{\prime}\right)\left\langle 0\left|T N_{8}\left[\partial_{i} \partial_{j} \varphi \partial_{i} \partial_{j} \varphi\right] X\right| 0\right\rangle+\left(q_{6}+p_{1} f_{6}+p_{2} f_{6}^{\prime}\right)\left\langle 0\left|T N_{8}\left[\Delta \partial_{i} \varphi \partial_{i} \varphi\right] X\right| 0\right\rangle \\
+ & \left(q_{7}+p_{1} f_{7}+p_{2} f_{7}^{\prime}\right)\left\langle 0\left|T N_{8}\left[\varphi^{4}\right] X\right| 0\right\rangle .
\end{aligned}
$$

Observe que podemos redefinir os parâmetros, por exemplo, $\bar{q}_{1} \equiv q_{1}+p_{1} f_{1}+p_{2} f_{1}^{\prime}$ e assim por diante, e esses novos parâmetros podem ser determinado diretamente a partir das condições de normalização.

\section{Condições de Normalização}

Seja $D^{M}$ um operador diferencial de grau $M \equiv z M_{0}+M_{1}$ nos momentos

$$
D^{M}=\frac{\partial^{M_{0}+M_{1}}}{\partial p_{1}^{0} \cdots \partial p_{M_{0}}^{0} \partial p_{M_{0}+1}^{i_{1}} \cdots \partial p_{M_{0}+M_{1}}^{i_{M_{1}}}} .
$$

Dessa forma,

$$
\left.D^{M} \Gamma_{\mathcal{O}}^{(N)}\left(p, p_{1}, \cdots, p_{N}\right)\right|_{p=p_{1}=\cdots=0}=\text { contribuição do diagrama trivial, }
$$

se $0 \leq M \leq \delta-2 N$, isto é, se a ordem do operador diferencial for menor ou igual ao grau do operador de Taylor. $\Gamma_{\mathcal{O}}^{(N)}\left(p, p_{1}, \cdots, p_{N}\right)$ é a função de vértice (amputada) de $N$ pontos com inserção do produto normal $N_{\delta}[\mathcal{O}(x)]$ :

$$
\begin{gathered}
(2 \pi)^{d+1} \delta\left(p+p_{1}+\cdots+p_{N}\right) \Gamma_{\mathcal{O}}^{(N)}\left(p, p_{1}, \cdots, p_{N}\right) \equiv \Delta^{-1}(p) \prod_{i=1}^{N} \Delta^{-1}\left(p_{i}\right) \\
\times \quad \int d x^{0} d^{d} x \prod_{i=1}^{N} d x_{i}^{0} d^{d} x_{i} e^{i\left(p x+\sum p_{k} x_{k}\right)}\left\langle 0\left|T N_{\delta}[\mathcal{O}(x)] \varphi\left(x_{1}\right) \cdots \varphi\left(x_{N}\right)\right| 0\right\rangle^{\text {prop }},
\end{gathered}
$$

lembrando que prop significa diagramas próprios $1 \mathrm{PI}$.

Como um exemplo, vamos determinar os parâmetros da identidade de Zimmermann (4.29). Primeiramente, de modo a determinar $f_{1}$, necessitamos aplicar o operador de 
quarta ordem $D^{4}=\partial^{2} / \partial p_{1}^{0} \partial p_{1}^{0}$ e então tomar os momentos externos iguais a zero. Após isso, os únicos termos que restam são: o termo acompanhando $f_{1}$, a saber, $\left\langle 0\left|T N_{8}\left[\varphi \partial_{0}^{2} \varphi\right] X\right| 0\right\rangle$, o qual tem apenas a contribuição do gráfico trivial e o termo envolvendo o produto normal reduzido $\left\langle 0\left|T N_{6}[\varphi \Delta \varphi] X\right| 0\right\rangle$. Com isso, encontramos

$$
f_{1}=\left.\frac{1}{2} \frac{\partial^{2}}{\partial p_{1}^{0} \partial p_{1}^{0}} \int d x d x_{1} d x_{2} e^{i p x+i p_{1} x_{1}+i p_{2} x_{2}}\left\langle 0\left|T N_{6}[\varphi \Delta \varphi](x) \varphi\left(x_{1}\right) \varphi\left(x_{2}\right)\right| 0\right\rangle^{\text {prop }}\right|_{p=p_{1}=p_{2}=0}
$$

sendo que definimos $d x \equiv d x_{0} d^{6} x$. Os outros termos podem ser determinados de maneira similar. A idéia é escolher operadores tais que, depois de sua aplicação sobre a identidade de Zimmermann, sobrevivam apenas os termos desejados. Assim, obtemos os seguintes resultados

$$
\begin{aligned}
f_{2}= & \left.\frac{1}{2} \frac{\partial^{2}}{\partial p_{1}^{0} \partial p_{2}^{0}} \int d x d x_{1} d x_{2} e^{i p x+i p_{1} x_{1}+i p_{2} x_{2}}\left\langle 0\left|T N_{6}[\varphi \Delta \varphi](x) \varphi\left(x_{1}\right) \varphi\left(x_{2}\right)\right| 0\right\rangle^{\text {prop }}\right|_{p=p_{1}=p_{2}=0}, \\
& 288 f_{3}+48 f_{5} \\
= & -\left.\frac{\partial^{4}}{\partial p_{1}^{i} \partial p_{1}^{i} \partial p_{2}^{j} \partial p_{2}^{j}} \int d x d x_{1} d x_{2} e^{i p x+i p_{1} x_{1}+i p_{2} x_{2}}\left\langle 0\left|T N_{6}[\varphi \Delta \varphi](x) \varphi\left(x_{1}\right) \varphi\left(x_{2}\right)\right| 0\right\rangle^{\text {prop }}\right|_{p=p_{1}=p_{2}=0}
\end{aligned}
$$

$$
\begin{aligned}
& 48 f_{3}+168 f_{5} \\
& =-\left.\frac{\partial^{4}}{\partial p_{1}^{i} \partial p_{2}^{i} \partial p_{1}^{j} \partial p_{2}^{j}} \int d x d x_{1} d x_{2} e^{i p x+i p_{1} x_{1}+i p_{2} x_{2}}\left\langle 0\left|T N_{6}[\varphi \Delta \varphi](x) \varphi\left(x_{1}\right) \varphi\left(x_{2}\right)\right| 0\right\rangle^{\text {prop }}\right|_{p=p_{1}=p_{2}=0},
\end{aligned}
$$

$f_{4}=-\left.\frac{1}{384} \frac{\partial^{4}}{\partial p_{1}^{i} \partial p_{1}^{i} \partial p_{1}^{j} \partial p_{1}^{j}} \int d x d x_{1} d x_{2} e^{i p x+i p_{1} x_{1}+i p_{2} x_{2}}\left\langle 0\left|T N_{6}[\varphi \Delta \varphi](x) \varphi\left(x_{1}\right) \varphi\left(x_{2}\right)\right| 0\right\rangle^{\text {prop }}\right|_{p=p_{1}=p_{2}=0}$,

$f_{6}=-\left.\frac{1}{96} \frac{\partial^{4}}{\partial p_{1}^{i} \partial p_{1}^{i} \partial p_{1}^{j} \partial p_{2}^{j}} \int d x d x_{1} d x_{2} e^{i p x+i p_{1} x_{1}+i p_{2} x_{2}}\left\langle 0\left|T N_{6}[\varphi \Delta \varphi](x) \varphi\left(x_{1}\right) \varphi\left(x_{2}\right)\right| 0\right\rangle^{\text {prop }}\right|_{p=p_{1}=p_{2}=0}$

$\mathrm{e}$

$$
f_{7}=-\left.\frac{1}{4 !} \int d x \prod_{i=1}^{N} d x_{i} e^{i p x+i \sum_{k} p_{k} x_{k}}\left\langle 0\left|T N_{6}[\varphi \Delta \varphi](x) \varphi\left(x_{1}\right) \varphi\left(x_{2}\right) \varphi\left(x_{3}\right) \varphi\left(x_{4}\right)\right| 0\right\rangle^{\text {prop }}\right|_{p=p_{1}=\cdots=p_{4}=0} .
$$

A partir dessas expressões podemos determinar a ordem na constante de acoplamento em que cada um dos termos começa a contribuir: $f_{i}=f_{i}^{(1)}+\cdots$, para $i=1, \ldots, 6$ e $f_{7}=f_{7}^{(2)}+\cdots$. Uma análise semelhante na identidade de Zimmermann (4.27) mostra 
que os fatores $p_{1}, p_{2}, q_{1}, \ldots, q_{6}$ começam em primeira ordem na constante de acoplamento e o fator $q_{7}$ em segunda ordem. Essa análise da ordem, bem como a dimensionalidade dos parâmetros envolvidos nas identidades de Zimmermann, serão importantes na determinação da anomalia de dilatações, como veremos em breve.

\section{Identidades de Zimmermann Integradas}

Antes de prosseguir, queremos discutir de maneira breve algumas simplificações que ocorrem quando consideramos produtos normais integrados. Isso porque podemos reduzir o número de produtos independentes fazendo integrações por partes. Assim, a identidade (4.27) torna-se

$$
\begin{aligned}
& \int d x^{0} d^{6} x\left\langle 0\left|T N_{8}\left[\varphi^{2}\right] X\right| 0\right\rangle=\int d x^{0} d^{6} x\left\langle 0\left|T N_{4}\left[\varphi^{2}\right] X\right| 0\right\rangle+p \int d x^{0} d^{6} x\left\langle 0\left|T N_{8}[\varphi \Delta \varphi] X\right| 0\right\rangle \\
+ & s \int d x^{0} d^{6} x\left\langle 0\left|T N_{8}\left[\partial_{0} \varphi \partial_{0} \varphi\right] X\right| 0\right\rangle+u \int d x^{0} d^{6} x\left\langle 0\left|T N_{8}\left[\varphi \Delta^{2} \varphi\right] X\right| 0\right\rangle \\
+ & v \int d x^{0} d^{6} x\left\langle 0\left|T N_{8}\left[\varphi^{4}\right] X\right| 0\right\rangle,
\end{aligned}
$$

em que redefinimos os parâmetros envolvidos. Do mesmo modo, para a identidade (4.29), temos

$$
\begin{aligned}
& \int d x^{0} d^{6} x\left\langle 0\left|T N_{8}[\varphi \Delta \varphi] X\right| 0\right\rangle=\int d x^{0} d^{6} x\left\langle 0\left|T N_{6}[\varphi \Delta \varphi] X\right| 0\right\rangle+g \int d x^{0} d^{6} x\left\langle 0\left|T N_{8}\left[\partial_{0} \varphi \partial_{0} \varphi\right] X\right| 0\right\rangle \\
& +k \int d x^{0} d^{6} x\left\langle 0\left|T N_{8}\left[\varphi \Delta^{2} \varphi\right] X\right| 0\right\rangle+l \int d x^{0} d^{6} x\left\langle 0\left|T N_{8}\left[\varphi^{4}\right] X\right| 0\right\rangle .
\end{aligned}
$$

Com essas relações, podemos obter a forma completamente reduzida de (4.42):

$$
\begin{aligned}
& \int d x^{0} d^{6} x\left\langle 0\left|T N_{8}\left[\varphi^{2}\right] X\right| 0\right\rangle=\int d x^{0} d^{6} x\left\langle 0\left|T N_{4}\left[\varphi^{2}\right] X\right| 0\right\rangle+p \int d x^{0} d^{6} x\left\langle 0\left|T N_{6}[\varphi \Delta \varphi] X\right| 0\right\rangle \\
& +\quad(s+p g) \int d x^{0} d^{6} x\left\langle 0\left|T N_{8}\left[\partial_{0} \varphi \partial_{0} \varphi\right] X\right| 0\right\rangle+(u+p k) \int d x^{0} d^{6} x\left\langle 0\left|T N_{8}\left[\varphi \Delta^{2} \varphi\right] X\right| 0\right\rangle \\
& +\quad(v+p l) \int d x^{0} d^{6} x\left\langle 0\left|T N_{8}\left[\varphi^{4}\right] X\right| 0\right\rangle .
\end{aligned}
$$

Os parâmetros são determinados a partir das condições de normalização. Para a identidade (4.43), segue que

$$
g=\left.\frac{1}{4} \frac{\partial^{2}}{\partial p_{1}^{0} \partial p_{1}^{0}} \int d x d x_{1} d x_{2} e^{i p_{1} x_{1}+i p_{2} x_{2}}\left\langle 0\left|T N_{6}[\varphi \Delta \varphi](x) \varphi\left(x_{1}\right) \varphi\left(x_{2}\right)\right| 0\right\rangle^{\text {prop }}\right|_{p_{1}=p_{2}=0}
$$




$$
k=-\left.\frac{1}{768} \frac{\partial^{4}}{\partial p_{1}^{i} \partial p_{1}^{i} \partial p_{1}^{j} \partial p_{1}^{j}} \int d x d x_{1} d x_{2} e^{i p_{1} x_{1}+i p_{2} x_{2}}\left\langle 0\left|T N_{6}[\varphi \Delta \varphi](x) \varphi\left(x_{1}\right) \varphi\left(x_{2}\right)\right| 0\right\rangle^{\text {prop }}\right|_{p_{1}=p_{2}=0}
$$

e

$$
l=-\left.\frac{1}{4 !} \int d x \prod_{i=1}^{N} d x_{i} e^{i \sum_{k} p_{k} x_{k}}\left\langle 0\left|T N_{6}[\varphi \Delta \varphi](x) \varphi\left(x_{1}\right) \varphi\left(x_{2}\right) \varphi\left(x_{3}\right) \varphi\left(x_{4}\right)\right| 0\right\rangle^{\text {prop }}\right|_{p_{1}=\cdots=p_{4}=0} .
$$

Dessas expressões, obtemos a ordem na constante de acoplamento que cada fator começa a contribuir: $g=g^{(2)}+\cdots, k=k^{(2)}+\cdots$ e $l=l^{(2)}+\cdots$. Uma análise semelhante em (4.42) mostra que $p, s, u, v$ são de segunda ordem no mínimo.

Agora, estamos prontos para discutir as leis de conservação ao nível quântico e então investigar a existência de anomalias. Vamos começar com invariância translacional.

\subsection{Tensor de Energia-Momento}

O tensor de energia-momento clássico pode ser construído a partir do teorema de Noether para teorias com altas derivadas aplicado a translações no espaço-tempo $x^{\mu} \rightarrow$ $x^{\mu}+\epsilon^{\mu}$. Necessitamos distinguir entre os seguintes casos:

a. Translações temporais. As componentes da corrente são

$$
\Theta_{00}=(1+A) \partial_{0} \varphi \partial_{0} \varphi-\mathcal{L}
$$

$\mathrm{e}$

$$
\bar{\Theta}_{i 0}=-\left(b^{2}+B\right) \partial_{i} \varphi \partial_{0} \varphi-\left(a^{2}+C\right) \Delta \varphi \partial_{i} \partial_{0} \varphi+\left(a^{2}+C\right)\left(\partial_{i} \Delta \varphi\right) \partial_{0} \varphi .
$$

b. Translações espaciais. Neste caso, temos as seguintes componentes

$$
\Theta_{0 i}=(1+A) \partial_{0} \varphi \partial_{i} \varphi
$$

$\mathrm{e}$

$$
\bar{\Theta}_{i j}=-\left(b^{2}+B\right) \partial_{i} \varphi \partial_{j} \varphi-\left(a^{2}+C\right) \Delta \varphi \partial_{i} \partial_{j} \varphi+\left(a^{2}+C\right)\left(\partial_{i} \Delta \varphi\right) \partial_{j} \varphi-\delta^{i j} \mathcal{L}
$$

Usando a equação de movimento clássica podemos verificar as leis de conservação

$$
\partial_{0} \Theta_{00}+\partial_{i} \bar{\Theta}_{i 0}=0 \text { and } \partial_{0} \Theta_{0 j}+\partial_{i} \bar{\Theta}_{i j}=0
$$

implicando na conservação da energia $H=\int d^{6} x \Theta_{00}$ e do momento $P_{i}=\int d^{6} x \Theta_{0 i}$.

Agora, queremos investigar esses aspectos na teoria quântica. Em primeiro lugar, as dimensões das componentes do tensor de energia-momento são: $\operatorname{Dim}\left[\Theta_{00}\right]=8, \operatorname{Dim}\left[\Theta_{0 i}\right]=$ 7, $\operatorname{Dim}\left[\bar{\Theta}_{i 0}\right]=9$ e $\operatorname{Dim}\left[\bar{\Theta}_{i j}\right]=8$. Assim, devemos construir os seguintes produtos normais 
$\left\langle 0\left|T N_{8}\left[\Theta_{00}\right] X\right| 0\right\rangle,\left\langle 0\left|T N_{7}\left[\Theta_{0 i}\right] X\right| 0\right\rangle,\left\langle 0\left|T N_{9}\left[\bar{\Theta}_{i 0}\right] X\right| 0\right\rangle$ e $\left\langle 0\left|T N_{8}\left[\bar{\Theta}_{i j}\right] X\right| 0\right\rangle$. De acordo com as regras dos produtos normais discutidas anteriormente, obtemos as leis de conservação análogas à (4.52) para o caso quântico:

$$
\partial_{0}\left\langle 0\left|T N_{8}\left[\Theta_{00}\right] X\right| 0\right\rangle+\partial_{i}\left\langle 0\left|T N_{9}\left[\bar{\Theta}_{i 0}\right] X\right| 0\right\rangle=-i \sum_{k} \delta\left(x-x_{k}\right)\left\langle 0\left|T \partial_{0} \varphi(x) X_{k}\right| 0\right\rangle
$$

e

$$
\partial_{0}\left\langle 0\left|T N_{7}\left[\Theta_{0 j}\right] X\right| 0\right\rangle+\partial_{i}\left\langle 0\left|T N_{8}\left[\bar{\Theta}_{i j}\right] X\right| 0\right\rangle=-i \sum_{k} \delta\left(x-x_{k}\right)\left\langle 0\left|T \partial_{j} \varphi(x) X_{k}\right| 0\right\rangle,
$$

em que $X_{k}$ é igual a $X$ mas com o campo $\varphi\left(x_{k}\right)$ omitido. Integrando sobre o espaço-tempo, o lado esquerdo se anula e encontramos o resultado

$$
\sum_{k}\left\langle 0\left|T \partial_{0} \varphi\left(x_{k}\right) X_{k}\right| 0\right\rangle=0 \quad \text { and } \quad \sum_{k}\left\langle 0\left|T \partial_{j} \varphi\left(x_{k}\right) X_{k}\right| 0\right\rangle=0 .
$$

Essas equações são as identidades de Ward que refletem a invariância das funções de Green sob translações no espaço-tempo.

\subsection{Anomalia de Dilatação}

\subsubsection{Análise Clássica}

A característica mais marcante das teorias estudadas neste trabalho é justamente o caráter anisotrópico entre as coordenadas temporal e espaciais: $\operatorname{Dim}\left[x_{0}\right]=z \mathrm{e} \operatorname{Dim}\left[x_{i}\right]=1$. O reflexo disso é que as dilatações, ou tranformações de escala globais, são da forma

$$
x_{0} \rightarrow\left(e^{\alpha}\right)^{z} x_{0} \quad \text { e } \quad x_{i} \rightarrow e^{\alpha} x_{i}
$$

Sob essa transformação, o campo escalar transforma-se como $\varphi(x) \rightarrow \varphi^{\prime}\left(x^{\prime}\right)=e^{-\alpha \operatorname{Dim}[\varphi]} \varphi(x)$, cuja versão infinitesimal com $\alpha \equiv-\epsilon$ é

$$
\delta \varphi(x)=\epsilon\left[\operatorname{Dim}[\varphi]+z x^{0} \partial_{0}+x^{i} \partial_{i}\right] \varphi(x) .
$$

Como é bastante conhecido no contexto de teorias relativísticas $(z=1)$, geralmente a invariância de escala global clássica é quebrada quânticamente pela necessidade da introdução de um parâmetro dimensional no procedimento de renormalização. Estudaremos a anomalia de dilatações nesse contexto de teorias anisotrópicas, começando com uma análise clássica. Por simplicidade, vamos considerar a lagrangiana (4.15) sem os contra-termos finitos,

$$
\mathcal{L}=\frac{1}{2} \partial_{0} \varphi \partial_{0} \varphi-\frac{b^{2}}{2} \partial_{i} \varphi \partial_{i} \varphi-\frac{a^{2}}{2} \Delta \varphi \Delta \varphi-\frac{m^{2}}{2} \varphi^{2}-\frac{\lambda}{4 !} \varphi^{4} .
$$


Os parâmetros dimensionais $b^{2}$ e $m^{2}$ na lagrangiana quebram a invariância de escala $\left(\operatorname{Dim}\left[m^{2}\right]=4\right.$ e $\left.\operatorname{Dim}\left[b^{2}\right]=2\right)$. Por outro lado, quando $b^{2}$ e $m^{2} \rightarrow 0$, temos uma corrente clássica conservada. Separando a lagrangiana em duas partes, $\mathcal{L}=\mathcal{L}_{\text {inv }}+\mathcal{L}_{b r}$, com

$$
\mathcal{L}_{i n v} \equiv \frac{1}{2} \partial_{0} \varphi \partial_{0} \varphi-\frac{a^{2}}{2} \Delta \varphi \Delta \varphi-\frac{\lambda}{4 !} \varphi^{4}
$$

$\mathrm{e}$

$$
\mathcal{L}_{b r} \equiv-\frac{b^{2}}{2} \partial_{i} \varphi \partial_{i} \varphi-\frac{m^{2}}{2} \varphi^{2}
$$

observamos que, sob uma transformação (4.56),

$$
\delta \mathcal{L}_{\text {inv }}=\epsilon\left[2 \partial_{0}\left(x^{0} \mathcal{L}_{\text {inv }}\right)+\partial_{i}\left(x^{i} \mathcal{L}_{\text {inv }}\right)\right],
$$

é uma soma de termos que são derivadas totais. Entretanto, o mesmo não ocorre com a parte de quebra:

$$
\delta \mathcal{L}_{b r}=\epsilon\left[2 \partial_{0}\left(x^{0} \mathcal{L}_{b r}\right)+\partial_{i}\left(x^{i} \mathcal{L}_{b r}\right)\right]-4 \epsilon\left(\frac{b^{2}}{2} \varphi \Delta \varphi-\frac{m^{2}}{2} \varphi^{2}\right) .
$$

A corrente pode ser construída sem dificuldades a partir do teorema de Noether e suas componentes são dadas por

$$
J_{0}=2 \varphi \partial_{0} \varphi+2 x^{0} \Theta_{00}+x^{i} \Theta_{0 i}
$$

$\mathrm{e}$

$$
J^{i}=-3 a^{2} \Delta \varphi \partial_{i} \varphi+2 a^{2}\left(\partial_{i} \Delta \varphi\right) \varphi-2 b^{2} \varphi \partial_{i} \varphi+2 x^{0} \bar{\Theta}_{i 0}+x^{j} \bar{\Theta}_{j i} .
$$

Note que estamos incluindo os termos de quebra. Com essas expressões, obtemos

$$
\partial_{0} J^{0}+\partial_{i} J^{i}=b^{2} \partial_{i} \varphi \partial_{i} \varphi+2 m^{2} \varphi^{2}
$$

tal que quando $m^{2}$ e $b^{2} \rightarrow 0$, temos a lei de conservação clássica. Por outro lado, no caso quântico devemos empregar um esquema de subtração para dar um significado preciso para expressões envolvendo operadores no mesmo ponto, o qual dá origem a anomalia.

\subsubsection{Análise Quântica}

Agora, vamos aplicar o método dos produtos normais para a corrente de dilatação. As dimensões das componentes da corrente são: $\operatorname{Dim}\left[J_{0}\right]=6$ e $\operatorname{Dim}\left[J_{i}\right]=7$. Desse modo, precisamos considerar os produtos normais $\left\langle 0\left|T N_{6}\left[J_{0}\right] X\right| 0\right\rangle$ e $\left\langle 0\left|T N_{7}\left[J_{i}\right] X\right| 0\right\rangle$. Desejamos investigar a lei de conservação

$$
\begin{array}{ll} 
& \partial_{0}\left\langle 0\left|T N_{6}\left[J_{0}\right] X\right| 0\right\rangle+\partial_{i}\left\langle 0\left|T N_{7}\left[J_{i}\right] X\right| 0\right\rangle \\
\stackrel{?}{=} & -i \sum_{k} \delta\left(x-x_{k}\right)\left\langle 0\left|T\left(\operatorname{Dim}[\varphi]+2 x^{0} \partial_{0}+x^{i} \partial_{i}\right) \varphi\left(x_{k}\right) X_{k}\right| 0\right\rangle .
\end{array}
$$


Vamos reescrever as componentes da corrente incluindo os contra-termos finitos

$$
J_{0}=d_{a}(1+A) \varphi \partial_{0} \varphi+2 x^{0} \Theta_{00}+x^{i} \Theta_{0 i}
$$

e

$$
J^{i}=-3\left(a^{2}+C\right) \Delta \varphi \partial_{i} \varphi+2\left(a^{2}+C\right)\left(\partial_{i} \Delta \varphi\right) \varphi-2\left(b^{2}+B\right) \varphi \partial_{i} \varphi+2 x^{0} \bar{\Theta}_{i 0}+x^{j} \bar{\Theta}_{j i},
$$

em que $d_{a}$ é a dimensão do campo escalar. Em ordem trivial de perturbação $d_{a}=$ $\operatorname{Dim}[\varphi]=2$, mas sabemos que no caso quântico ela sofre correções radiativas, quer dizer, $d_{a}=2+\mathcal{O}(\lambda)$. Para avaliar (4.66) é conveniente usar as identidades

$$
\left\langle 0\left|T N_{8}\left[x^{i} \partial_{0} \Theta_{0 i}\right] X\right| 0\right\rangle=x^{i} \partial_{0}\left\langle 0\left|T N_{7}\left[\Theta_{0 i}\right] X\right| 0\right\rangle
$$

$\mathrm{e}$

$$
\left\langle 0\left|T N_{8}\left[x^{0} \partial_{i} \bar{\Theta}_{i 0}\right] X\right| 0\right\rangle=x^{0} \partial_{i}\left\langle 0\left|T N_{9}\left[\bar{\Theta}_{i 0}\right] X\right| 0\right\rangle .
$$

Além disso, empregando as relações de conservação do tensor de energia-momento (4.53) e (4.54) e a equação de movimento (4.25), obtemos

$$
\begin{aligned}
& \partial_{0}\left\langle 0\left|T N_{6}\left[J_{0}\right] X\right| 0\right\rangle+\partial_{i}\left\langle 0\left|T N_{7}\left[J_{i}\right] X\right| 0\right\rangle=\left(d_{a}-2\right)(1+A)\left\langle 0\left|T N_{8}\left[\partial_{0} \varphi \partial_{0} \varphi\right] X\right| 0\right\rangle \\
+ & \left(d_{a}-2\right)\left(b^{2}+B\right)\left\langle 0\left|T N_{8}[\varphi \Delta \varphi] X\right| 0\right\rangle-\left(d_{a}-2\right)\left(a^{2}+C\right)\left\langle 0\left|T N_{8}\left[\varphi \Delta^{2} \varphi\right] X\right| 0\right\rangle \\
- & \left(d_{a}-4\right)\left(m^{2}+D\right)\left\langle 0\left|T N_{8}\left[\varphi^{2}\right] X\right| 0\right\rangle-\left(d_{a}-2\right) \frac{(\lambda+E)}{3 !}\left\langle 0\left|T N_{8}\left[\varphi^{4}\right] X\right| 0\right\rangle \\
+ & \left(b^{2}+B\right)\left\langle 0\left|T N_{8}\left[\partial_{i} \varphi \partial \varphi\right] X\right| 0\right\rangle-i \sum_{k} \delta\left(x-x_{k}\right)\left\langle 0\left|T\left(d_{a}+2 x^{0} \partial_{0}+x^{i} \partial_{i}\right) \varphi\left(x_{k}\right) X_{k}\right| 0\right\rangle .
\end{aligned}
$$

Integrando sobre o espaço-tempo, essa expressão torna-se

$$
\begin{aligned}
& i \sum_{k}\left\langle 0\left|T\left(d_{a}+2 x^{0} \partial_{0}+x^{i} \partial_{i}\right) \varphi\left(x_{k}\right) X_{k}\right| 0\right\rangle=\int d x^{0} d^{6} x\left\{\left(d_{a}-2\right)(1+A)\left\langle 0\left|T N_{8}\left[\partial_{0} \varphi \partial_{0} \varphi\right] X\right| 0\right\rangle\right. \\
& +\quad\left(d_{a}-3\right)\left(b^{2}+B\right)\left\langle 0\left|T N_{8}[\varphi \Delta \varphi] X\right| 0\right\rangle-\left(d_{a}-2\right)\left(a^{2}+C\right)\left\langle 0\left|T N_{8}\left[\varphi \Delta^{2} \varphi\right] X\right| 0\right\rangle \\
& \left.-\quad\left(d_{a}-4\right)\left(m^{2}+D\right)\left\langle 0\left|T N_{8}\left[\varphi^{2}\right] X\right| 0\right\rangle-\left(d_{a}-2\right) \frac{(\lambda+E)}{3 !}\left\langle 0\left|T N_{8}\left[\varphi^{4}\right] X\right| 0\right\rangle\right\} .
\end{aligned}
$$

Podemos usar as identidades de Zimmermann (4.43) e (4.44) para reduzir os produtos normais com subtrações adicionais $\left\langle 0\left|T N_{8}[\varphi \Delta \varphi] X\right| 0\right\rangle$ e $\left\langle 0\left|T N_{8}\left[\varphi^{2}\right] X\right| 0\right\rangle$. O resultado final 
é

$$
\begin{aligned}
& i \sum_{k}\left\langle 0\left|T\left(d_{a}+2 x^{0} \partial_{0}+x^{i} \partial_{i}\right) \varphi\left(x_{k}\right) X_{k}\right| 0\right\rangle=\int d x^{0} d^{6} x\left\{-\left(d_{a}-4\right)\left(m^{2}+D\right)\left\langle 0\left|T N_{4}\left[\varphi^{2}\right] X\right| 0\right\rangle\right. \\
& +\left[\left(d_{a}-3\right)\left(b^{2}+B\right)-\left(d_{a}-4\right)\left(m^{2}+D\right) p\right]\left\langle 0\left|T N_{6}[\varphi \Delta \varphi] X\right| 0\right\rangle \\
& +\left[\left(d_{a}-2\right)(1+A)+\left(d_{a}-3\right)\left(b^{2}+B\right) g-\left(d_{a}-4\right)\left(m^{2}+D\right) \bar{s}\right]\left\langle 0\left|T N_{8}\left[\partial_{0} \varphi \partial_{0} \varphi\right] X\right| 0\right\rangle \\
& +\left[-\left(d_{a}-2\right)\left(a^{2}+C\right)+\left(d_{a}-3\right)\left(b^{2}+B\right) k-\left(d_{a}-4\right)\left(m^{2}+D\right) \bar{u}\right]\left\langle 0\left|T N_{8}\left[\varphi \Delta^{2} \varphi\right] X\right| 0\right\rangle \\
& \left.+\left[-\left(d_{a}-2\right) \frac{(\lambda+E)}{3 !}+\left(d_{a}-3\right)\left(b^{2}+B\right) l-\left(d_{a}-4\right)\left(m^{2}+D\right) \bar{v}\right]\left\langle 0\left|T N_{8}\left[\varphi^{4}\right] X\right| 0\right\rangle\right\},
\end{aligned}
$$

em que definimos: $\bar{s} \equiv s+p g, \bar{u} \equiv u+p k$ e $\bar{v} \equiv v+p l$. Para que não exista anomalia na corrente, os coeficientes dos três produtos normais de grau $N_{8}$ devem se anular independentemente, o que conduz às seguintes condições

$$
\begin{array}{r}
\left(d_{a}-2\right)(1+A)+\left(d_{a}-3\right)\left(b^{2}+B\right) g-\left(d_{a}-4\right)\left(m^{2}+D\right) \bar{s}=0 \\
-\left(d_{a}-2\right)\left(a^{2}+C\right)+\left(d_{a}-3\right)\left(b^{2}+B\right) k-\left(d_{a}-4\right)\left(m^{2}+D\right) \bar{u}=0
\end{array}
$$

$\mathrm{e}$

$$
-\left(d_{a}-2\right) \frac{(\lambda+E)}{3 !}+\left(d_{a}-3\right)\left(b^{2}+B\right) l-\left(d_{a}-4\right)\left(m^{2}+D\right) \bar{v}=0 .
$$

Vamos discutir a consistência dessas equações. Em primeiro lugar, lembramos que os termos $g, \bar{s}, k, \bar{u}, l$ e $\bar{v}$ são todos de segunda ordem na constante de acoplamento. Portanto, escrevendo $d_{a}=2+d_{a}^{(1)}+d_{a}^{(2)}+\cdots$, a equação (4.74) mostra que $d_{a}^{(1)}=0$. Explicitando a ordem de cada termo, obtemos as condições de consistência em ordem mais baixa

$$
\begin{gathered}
d_{a}^{(2)}-b^{2} g^{(2)}+2 m^{2} \bar{s}^{(2)}=0, \\
-a^{2} d_{a}^{(2)}-b^{2} k^{(2)}+2 m^{2} \bar{u}^{(2)}=0
\end{gathered}
$$

$\mathrm{e}$

$$
-b^{2} l^{(2)}+2 m^{2} \bar{v}^{(2)}=0
$$

Devemos investigar essas condições no limite $b^{2}, m^{2} \rightarrow 0$. Ingenuamente, isso parece resolver essas equações $\left(\operatorname{com} d_{a}^{(2)}=0\right)$, mas devemos ser cuidadosos. Relembrando nossa análise dimensional, temos, por exemplo, $\operatorname{Dim}[g]=-2$, a qual significa que $g^{(2)}$ pode ser escrita como $g^{(2)}=1 / b^{2} \mathcal{F}\left(m^{2} / b^{4}, a^{2}\right) \lambda^{2}$, em que $\mathcal{F}$ é uma função adimensional dos parâmetros originais da teoria. Isso também pode ser visto de um cálculo explícito. Então, o produto $b^{2} g^{(2)}$ não se anula quando $b^{2}, m^{2} \rightarrow 0$, com $m^{2} / b^{4} \rightarrow$ fixo. O termo de massa tem um comportamento similar: como $\operatorname{Dim}[\bar{s}]=-4, \bar{s}^{(2)}$ pode ser escrita como $\bar{s}^{(2)}=1 / m^{2} \mathcal{G}\left(m^{2} / b^{4}, a^{2}\right) \lambda^{2}$ e então o produto $m^{2} \bar{s}^{(2)}$ não se anula no limite $b^{2}, m^{2} \rightarrow 0$, com $m^{2} / b^{4} \rightarrow$ fixo. O mesmo fato ocorre com os demais termos nas equações (4.78) e (4.79). 
Para ilustrar essa questão, vamos considerar, por exemplo, a condição (4.79) no limite $b^{2}, m^{2} \rightarrow 0$, mas $m^{2} / b^{4} \equiv c>0$, com $c$ um número real positivo. Isso é suficiente para verificar a existência da anomalia na corrente. A expressão para $l^{(2)}$ é dada em (4.47), ao passo que a expressão para $\bar{v}^{(2)}$ tem a mesma forma que (4.47), porém com a troca do produto normal $N_{6}[\varphi \Delta \varphi]$ por $N_{4}\left[\varphi^{2}\right]$. Dessa forma, a expressão explícita do lado esquerdo da equação (4.79) é

$$
\frac{6 i^{3} \lambda^{2}}{4 !} \int \frac{d k_{0}}{2 \pi} \frac{d^{6} k}{(2 \pi)^{6}} \frac{\left(b^{2} \mathbf{k}^{2}+2 m^{2}\right)}{\left[k_{0}^{2}-b^{2} \mathbf{k}^{2}-a^{2}\left(\mathbf{k}^{2}\right)^{2}-m^{2}+i \epsilon\right]^{3}} .
$$

Assim, vamos investigar a possibilidade do anulamento dessa equação no limite acima mencionado. Tomando o limite, segue que

$$
\frac{6 i^{3} \lambda^{2}}{4 !} \int \frac{d k_{0}}{2 \pi} \frac{d^{6} k}{(2 \pi)^{6}} \frac{\left(\mathbf{k}^{2}+2 c\right)}{\left[k_{0}^{2}-\mathbf{k}^{2}-a^{2}\left(\mathbf{k}^{2}\right)^{2}-c+i \epsilon\right]^{3}} .
$$

Agora, podemos integrar na componente $k_{0}$ via o teorema dos resíduos:

$$
\frac{9}{8} \frac{\lambda^{2}}{4 !} \int \frac{d^{6} k}{(2 \pi)^{6}} \frac{\left(\mathbf{k}^{2}+2 c\right)}{\left[\mathbf{k}^{2}+a^{2}\left(\mathbf{k}^{2}\right)^{2}+c\right]^{\frac{5}{2}}}
$$

Parametrizando em coordenadas esféricas e fazendo a mudança de variável $q \equiv k^{2}$, obtemos

$$
\frac{9}{16} \frac{\lambda^{2}}{4 !} \frac{\Omega_{6}}{(2 \pi)^{6}} \int_{0}^{\infty} d q \frac{\left(q^{3}+2 c q^{2}\right)}{\left(q+a^{2} q^{2}+c\right)^{\frac{5}{2}}}=\frac{1}{4 !} \frac{3}{512 \pi^{3}} \frac{\lambda^{2}}{a^{3}} \neq 0,
$$

a qual é não nula para qualquer valor finito de $a . \Omega_{6}$ é área da superfície esférica em seis dimensões, $\Omega_{6}=2 \pi^{3} / \Gamma(3)$. Isso mostra que a equação (4.79) não pode ser satisfeita, conduzindo ao comportamento anômalo da corrente quântica. Um último comentário é que a divergência da corrente de dilatação deve ser proporcional à função beta do grupo de renormalização. De fato, podemos mostrar que ela é dada essencialmente pelo lado esquerdo de (4.79),

$$
\beta(\lambda)=4 !\left(-b^{2} l^{(2)}+2 m^{2} \bar{v}^{(2)}\right)=\frac{3}{512 \pi^{3}} \frac{\lambda^{2}}{a^{3}},
$$

que é exatamente o resultado obtido em (2.136).

Antes de concluir, queremos apenas destacar que neste capítulo fizemos a generalização do método dos produtos normais, desenvolvidos inicialmente no contexto de teorias relativísticas, para o caso de teorias com anisotropia. O caso relativístico corresponde a $z=1$. Esse formalismo é direcionado para o estudo de simetrias quânticas em geral a partir da construção dos geradores correspondentes, possibilitando uma análise sistemática das identidades de Ward e possíveis anomalias. Estudos sobre as anomalias quiral e de Weyl em teorias anisotrópicas foram feitos em [52, 53, 54, 55]. 


\section{Capítulo 5}

\section{Modelos Estatísticos Clássicos}

Neste capítulo, discutiremos alguns aspectos do modelo de Ising, como a solução exata em uma dimensão, a dualidade entre altas e baixas temperaturas em duas dimensões e a conexão com a teoria de campos. Em seguida, consideraremos o modelo esférico clássico que, juntamente com o modelo de Ising, desempenha um papel fundamental no estudo de transições de fase principalmente pelo fato de ser exatamente solúvel e constitui o ponto de partida das generalizações contidas nos capítulos seguintes.

\subsection{Modelo de Ising}

O modelo de Ising é definido sobre uma rede hipercúbica $d$-dimensional com $N$ sítios e a cada sítio associamos uma variável de spin discreta $\sigma_{i}= \pm 1$. A hamiltoniana na presença de um campo externo constante $h$ é dada por

$$
\mathcal{H}=-J \sum_{<i, j>} \sigma_{i} \sigma_{j}-h \sum_{i} \sigma_{i},
$$

em que o símbolo $\langle i, j>$ indica que a soma deve ser restrita aos primeiros vizinhos (curto alcance) com uma energia de interação ferromagnética $J>0$. Além disso, vamos assumir condições periódicas de contorno $\sigma_{N+1} \equiv \sigma_{1}$, o que não deve influenciar no comportamento do sistema no limite termodinâmico. Apesar da simplicidade da formulação, o cálculo da função de partição se torna mais elaborado a medida que aumentamos a dimensionalidade da rede. De fato, em uma dimensão espacial o modelo é exatamente solúvel na presença de campo externo, mas não exibe uma transição de fase. Em duas dimensões, ele tem uma solução exata na ausência de campo (solução de Onsager) e em três dimensões não possui solução exata mesmo na ausência de campo. 
A solução em uma dimensão é bastante simples e vamos determiná-la agora pelo método da matriz de transferência. Nesse caso, a hamiltoniana (5.1) se reduz a

$$
\mathcal{H}=-J \sum_{i} \sigma_{i} \sigma_{i+1}-\frac{h}{2} \sum_{i}\left(\sigma_{i}+\sigma_{i+1}\right)
$$

em que a parte dependente do campo externo foi escrita convenientemente numa forma mais simétrica, o que é possível pela escolha da condição de contorno. Devemos calcular a função de partição, que envolve uma soma sobre as possíveis configurações $\{\sigma\}$ do sistema distribuídas de acordo com o peso de Boltzmann

$$
Z=\sum_{\{\sigma\}} e^{-\beta \mathcal{H}}
$$

Podemos deixá-la numa forma particularmente simples definindo a matriz $T$, denominada matriz de transferência,

$$
T_{\sigma_{i}, \sigma_{i+1}} \equiv e^{\beta J \sigma_{i} \sigma_{i+1}+\frac{\beta h}{2}\left(\sigma_{i}+\sigma_{i+1}\right)} \Rightarrow T=\left(\begin{array}{cc}
e^{\beta J+\beta h} & e^{-\beta J} \\
e^{-\beta J} & e^{\beta J-\beta h}
\end{array}\right) .
$$

Assim, a função de partição pode ser identificada como o traço de um produto de matrizes $T$

$$
Z=\sum_{\sigma_{1}} \cdots \sum_{\sigma_{N}} T_{\sigma_{1}, \sigma_{2}} T_{\sigma_{2}, \sigma_{3}} \cdots T_{\sigma_{N}, \sigma_{1}}=\operatorname{Tr} T^{N}
$$

Como a matriz de transferência é simétrica, existe uma transformação envolvendo uma matriz $U$ que a diagonaliza, isto é, $D \equiv U T U^{-1}$ com

$$
D \equiv\left(\begin{array}{cc}
\lambda_{1} & 0 \\
0 & \lambda_{2}
\end{array}\right)
$$

e $\lambda_{1}$ e $\lambda_{2}$ são os autovalores de $T$. Como o traço é invariante pela transformação acima, a função de partição torna-se

$$
Z=\operatorname{Tr} D^{N}=\lambda_{1}^{N}+\lambda_{2}^{N}
$$

Os autovalores são dados por

$$
\lambda_{1,2}=e^{\beta J} \cosh (\beta h) \pm \sqrt{e^{2 \beta J} \cosh ^{2}(\beta h)-2 \sinh (2 \beta J)} .
$$

Note que $\lambda_{1}>\lambda_{2}$, tal que no limite termodinâmico apenas o maior autovalor contribui

$$
Z=\lim _{N \rightarrow \infty} \lambda_{1}^{N}\left[1+\left(\frac{\lambda_{2}}{\lambda_{1}}\right)^{N}\right] \rightarrow \lambda_{1}^{N}
$$


A conexão com a termodinâmica é feita por meio da energia livre

$$
f=-\frac{1}{\beta N} \ln Z=-\frac{1}{\beta} \ln \lambda_{1} .
$$

A partir dessa expressão, podemos calcular a magnetização por spin de acordo com

$$
m=-\left(\frac{\partial f}{\partial h}\right)_{\beta}=\frac{\sinh (\beta h)}{\sqrt{\sinh ^{2}(\beta h)+e^{-4 \beta J}}},
$$

que se anula quando $h=0$, mostrando que o sistema não exibe uma magnetização espontânea na ausência de campo, ou seja, não exibe uma transição de fase. A idéia intuitiva por trás disso é que em uma dimensão espacial o número de primeiros vizinhos de cada sítio é pequeno (dois) tal que o sistema não tem "força"para formar um estado ordenado.

Consideremos agora o caso bidimensional. Ao invés de estudar a solução exata na rede quadrada, que apesar do grande interessante é demasiadamente complicada, faremos a análise de uma dualidade existente nesse caso e que permite determinar a temperatura crítica do modelo.

A função de partição (5.1) na ausência de campo externo pode ser escrita como

$$
Z=\sum_{\{\sigma\}} \prod_{<i, j>} e^{K \sigma_{i} \sigma_{j}}
$$

em que definimos $K \equiv \beta J$. Devido ao caráter discreto das variáveis $\sigma_{i}= \pm 1$, segue que

$$
\begin{aligned}
e^{K \sigma_{i} \sigma_{j}} & =\cosh K+\sigma_{i} \sigma_{j} \sinh K \\
& =\cosh K\left(1+\sigma_{i} \sigma_{j} \tanh K\right) .
\end{aligned}
$$

Dessa forma, a função de partição (5.12) torna-se

$$
Z=(\cosh K)^{2 N} \sum_{\{\sigma\}} \prod_{<i, j>}\left(1+\sigma_{i} \sigma_{j} \tanh K\right),
$$

em que $2 N$ é o número total de primeiros vizinhos. Para uma rede hipercúbia $d$-dimensional esse número tem a forma geral $2 d \times N / 2$; o fator $2 d$ é o número de coordenação da rede (número de primeiros vizinhos em $d$ dimensões), $N$ é o número total de sítios e a divisão pelo fator de 2 é para não contar duas vezes o mesmo par de primeiros vizinhos. A expressão acima ainda pode ser escrita como

$$
\begin{aligned}
Z & =(\cosh K)^{2 N} \sum_{\{\sigma\}} \prod_{<i, j>} \sum_{l=0}^{1}\left(\sigma_{i} \sigma_{j} \tanh K\right)^{l} \\
& =(\cosh K)^{2 N} \sum_{\{\sigma\}}\left(\sum_{l=0}^{1}\left(\sigma_{1} \sigma_{2} \tanh K\right)^{l}\right) \times\left(\sum_{l=0}^{1}\left(\sigma_{2} \sigma_{3} \tanh K\right)^{l}\right) \times \cdots
\end{aligned}
$$


É conveniente introduzir a variável $l_{k} \equiv l_{i j}=l_{j i}=0,1$ para cada par de primeiros vizinhos, que representa cada ligação entre dois sítios mais próximos. Dessa forma,

$$
Z=(\cosh K)^{2 N} \sum_{\{\sigma\}} \sum_{l_{1}} \cdots \sum_{l_{2 N}}(\tanh K)^{l_{1}+l_{2}+\cdots} \prod_{<i, j>}\left(\sigma_{i} \sigma_{j}\right)^{l_{i j}}
$$

Observe que o produtório sobre os pares de primeiros vizinhos pode ser rearranjado da seguinte forma

$$
\prod_{<i, j>}\left(\sigma_{i} \sigma_{j}\right)^{l_{i j}}=\prod_{i} \sigma_{i}^{\sum_{j} l_{i j}}=\prod_{i} \sigma_{i}^{n_{i}},
$$

em que definimos $n_{i} \equiv \sum_{j} l_{i j}$, com $j$ referindo-se aos primeiros vizinhos do sítio $i$. Essa relação pode ser melhor entendida a partir de um exemplo: considere um sítio $a$ e os seus primeiros vizinhos $a_{1}, a_{2}, a_{3}$ e $a_{4}$. Começando com o lado esquerdo, a parte do produto acima envolvendo esse sítio particular será

$$
\begin{aligned}
& \cdots\left(\sigma_{a} \sigma_{a_{1}}\right)^{l_{a a_{1}}}\left(\sigma_{a} \sigma_{a_{2}}\right)^{l_{a a_{2}}}\left(\sigma_{a} \sigma_{a_{3}}\right)^{l_{a a_{3}}}\left(\sigma_{a} \sigma_{a_{4}}\right)^{l_{a a_{4}}} \ldots \\
= & \cdots\left(\sigma_{a}\right)^{l_{a a_{1}}+l_{a a_{2}}+l_{a a_{3}}+l_{a a_{4}}}\left(\sigma_{a_{1}}\right)^{l_{a a_{1}}}\left(\sigma_{a_{2}}\right)^{l_{a a_{2}}}\left(\sigma_{a_{3}}\right)^{l_{a a_{3}}}\left(\sigma_{a_{4}}\right)^{l_{a a_{4}}} \ldots .
\end{aligned}
$$

Os fatores envolvendo a variável de spin do sítio a foram deixados na forma do lado direito de (5.17). Os termos restantes, envolvendo as variáveis dos sítios $a_{1}, a_{2}, a_{3}$ e $a_{4}$ também serão organizadas em uma estrutura do mesmo tipo quando levarmos em conta os primeiros vizinhos correspondentes a esses sítios. Esse processo pode ser feito para a rede inteira conduzindo ao resultado (5.17). Com isso, podemos calcular a soma sobre $\{\sigma\}$

$$
\sum_{\{\sigma\}} \prod_{<i, j>}\left(\sigma_{i} \sigma_{j}\right)^{l_{i j}}=\sum_{\{\sigma\}} \prod_{i} \sigma_{i}^{n_{i}}=\prod_{i}\left(1+(-1)^{n_{i}}\right) .
$$

Quando pelo menos um $n_{i}$ é ímpar essa relação se anula. Por outro lado, se todos $n_{i}$ forem pares o resultado é

$$
\prod_{i} 2=2^{N}
$$

Com essas observações, a função de partição fica

$$
Z=\left(2 \cosh ^{2} K\right)^{N} \sum_{\{l\}}(\tanh K)^{l_{1}+l_{2}+\cdots},
$$

sob o vínculo

$$
\sum_{j} l_{i, j}=0,2 \text { ou } 4
$$

para quaisquer quatro ligações juntando-se em um determinado sítio. Nossa estratégia é introduzir um novo conjunto de variáveis de modo que o vínculo seja automaticamente satisfeito. Para isso, vamos considerar uma rede dual construída a partir da rede original, 
mostradas na figura 5.1. Associamos então a cada sítio da rede dual a variável $\chi_{i}=$ \pm 1 . Como pode ser visto na figura 5.1, dois sítios primeiros vizinhos da rede dual são "cortados" por uma ligação $l_{k}$ da rede original. Para um sítio particular, o qual tem as ligações $l_{1}, l_{2}, l_{3}$ e $l_{4}$ conectando os seus primeiros vizinhos, essas relações são definidas como

$$
\begin{aligned}
l_{1} & \equiv \frac{1}{2}\left(1-\chi_{1} \chi_{2}\right), \quad l_{2} \equiv \frac{1}{2}\left(1-\chi_{2} \chi_{3}\right), \\
l_{3} \equiv \frac{1}{2}\left(1-\chi_{3} \chi_{4}\right), & l_{4} \equiv \frac{1}{2}\left(1-\chi_{4} \chi_{1}\right) .
\end{aligned}
$$

Com isso, é imediato verificar que o vínculo é satisfeito

$$
l_{1}+l_{2}+l_{3}+l_{4}=\frac{1}{2}\left[4-\left(\chi_{1}+\chi_{3}\right)\left(\chi_{2}+\chi_{4}\right)\right]=0,2 \text { ou } 4 .
$$

Essa construção deve ser feita para todos os sítios da rede e assim o vínculo (5.22) será naturalmente implementado por meio das variáveis da rede dual. Voltando a função de

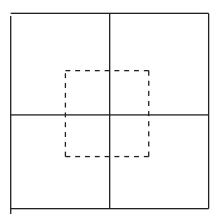

Figura 5.1: Relação entre a rede original e a rede dual. As linhas contínuas formam a rede original e as linhas tracejadas formam a rede dual.

partição, podemos escrever cada um dos fatores (tanh $K)^{l_{k}}$ em termos das variáveis da rede dual $\chi_{i}$ e $\chi_{j}$ que são cortadas pela ligação $l_{k}$ (consequentemente $i$ e $j$ são primeiros vizinhos na rede dual):

$$
(\tanh K)^{l_{k}}=(\tanh K)^{\frac{1}{2}\left(1-\chi_{i} \chi_{j}\right)} \equiv e^{-K^{*}\left(1-\chi_{i} \chi_{j}\right)},
$$

em que definimos

$$
\tanh K \equiv e^{-2 K^{*}}
$$

Assim, a função de partição (5.21) torna-se

$$
Z(K)=\left(2 \cosh ^{2} K\right)^{N} e^{-2 N K^{*}} \sum_{\{\chi\}} e^{K^{*} \sum_{<i, j>} \chi_{i} \chi_{j}} .
$$

Devemos fazer algumas observações importantes. A primeira é que nessa expressão podemos identificar a função de partição da rede dual, que tem a mesma forma que a função de partição inicial (5.12), porém com $K^{*}$ no lugar de $K$ :

$$
Z\left(K^{*}\right)=\sum_{\{\chi\}} e^{K^{*} \sum_{<i, j>} \chi_{i} \chi_{j}} .
$$


Assim, as funções de partição da rede original e da rede dual estão relacionadas de acordo $\operatorname{com}(5.27)$

$$
\frac{Z(K)}{\left(2 \cosh ^{2} K\right)^{N}}=\frac{Z\left(K^{*}\right)}{\left(e^{2 K^{*}}\right)^{N}}
$$

A relação entre $K$ e $K^{*}$ é dada em (5.26), que pode ser escrita de uma maneira mais conveniente como

$$
\sinh (2 K) \sinh \left(2 K^{*}\right)=1
$$

Essa equação pode ser interpretada como definindo uma transformação entre altas e baixas temperaturas. Por exemplo, se $K \rightarrow 0$, então $K^{*} \rightarrow \infty$; caso contrário, quando $K \rightarrow \infty$, devemos ter $K^{*} \rightarrow 0$. Em outras palavras, o comportamento de altas temperaturas na rede original corresponde a um comportamento de baixas temperaturas na rede dual. É natural imaginar que há um ponto especial quando esses regimes se encontram, ou seja, quando $K=K^{*} \equiv K_{c}$, dito o ponto auto-dual. De fato, escrevendo $K_{c}=J \beta_{c}$, esse ponto é identificado como a temperatura crítica do modelo de Ising na rede quadrada

$$
\beta_{c} J=\frac{1}{2} \ln (\sqrt{2}+1)
$$

concordando exatamente com o resultado obtido a partir da solução exata de Onsager.

\subsubsection{Conexão com a Teoria de Campos}

Usualmente, os modelos estatísticos são formulados sobre redes cristalinas, que são estruturas discretas caracterizadas pela sua geometria e pelo espaçamento entre seus vizinhos. Uma questão natural que surge está relacionada ao limite contínuo da rede. Nessa situação, teremos ao invés da rede um espaço contínuo, revelando uma teoria de campos subjacente. Essa teoria de campos pode ser interpretada como uma descrição efetiva nas proximidades do ponto crítico, em que o comprimento de correlação é muito maior que a distância entre os sítios.

Queremos investigar o limite contínuo da rede hipercúbica $d$-dimensional sobre a qual o modelo de Ising é definido. Como as variáveis de Ising $\sigma_{i}$ são discretas, isso não pode ser feito de uma maneira direta como na seção 1.3. mas seguiremos um procedimento semelhante. Em primeiro lugar, reescrevemos a função de partição na ausência de campo como

$$
Z=\sum_{\{\sigma\}} \exp \left[\sum_{i j} J_{i, j} \sigma_{i} \sigma_{j}\right]
$$

Nessa notação, a interação de primeiros vizinhos está codificada em $J_{i, j}$, a qual também inclui o fator $\beta$. Agora, considere a seguinte identidade

$$
\int \prod_{i} d \phi_{i} \exp \left[-\frac{1}{4} \sum_{i, j} \phi_{i} J_{i j}^{-1} \phi_{j}+\sum_{i} \phi_{i} \sigma_{i}\right]=A \exp \left[\sum_{i j} J_{i, j} \sigma_{i} \sigma_{j}\right],
$$


em que $A$ é uma constante de normalização irrelevante e $\left\{\phi_{i}\right\}$ é um conjunto de variáveis contínuas. Essa identidade nos possibilita escrever a função de partição como

$$
\begin{aligned}
Z & =\int \prod_{i} d \phi_{i} \sum_{\{\sigma\}} \exp \left[-\frac{1}{4} \sum_{i, j} \phi_{i} J_{i j}^{-1} \phi_{j}+\sum_{i} \phi_{i} \sigma_{i}\right] \\
& =\int \prod_{i} d \phi_{i} \exp \left[-\frac{1}{4} \sum_{i, j} \phi_{i} J_{i j}^{-1} \phi_{j}+\sum_{i} \ln \left(\cosh \phi_{i}\right)\right],
\end{aligned}
$$

a menos de constantes multiplicativas. Com isso, conseguimos passar de um conjunto de variáveis discretas para um conjunto de variáveis contínuas. Ainda podemos fazer uma mudança de variável para deixá-la numa forma mais adequada. Fazendo,

$$
\phi_{i} \rightarrow 2 \sum_{j} J_{i j} \phi_{i}
$$

obtemos

$$
Z=\int \prod_{i} d \phi_{i} \exp \left[-\sum_{i, j} J_{i j} \phi_{i} \phi_{j}+\sum_{i} \ln \left[\cosh \left(2 \sum_{j} J_{i j} \phi_{j}\right)\right]\right]
$$

Nessa situação, em que a função de partição envolve apenas variáveis contínuas, o limite contínuo se torna mais transparente. De fato, observe que para o caso de interações de primeiros vizinhos $J_{i j}=J \delta_{i, j=i \pm a}$, com $a$ o espaçamento da rede, temos

$$
\left.\sum_{j} J_{i j} \phi_{j} \sim J a^{2} \nabla^{2} \phi\right|_{i}+\left.2 d J \phi\right|_{i}
$$

Além disso, as somatórias podem ser substituídas por integrais $\sum_{i} \rightarrow a^{-d} \int d^{d} x$. Dessa forma, a versão contínua de (5.36) é dada por

$$
Z=\int \mathcal{D} \phi \exp \left[-\int \frac{d^{d} x}{a^{d}}\left(J a^{2} \phi \nabla^{2} \phi+2 d J \phi^{2}\right)+\int \frac{d^{d} x}{a^{d}} \ln \left[\cosh \left(2 J a^{2} \nabla^{2} \phi+4 d J \phi\right)\right]\right],
$$

que pode ser interpretada como a teoria de campos surgindo no limite contínuo da rede. Entretanto, a expressão acima é bastante complicada tal que podemos deixá-la numa forma polinomial fazendo a expansão

$$
\ln \cosh x=\frac{x^{2}}{2}-\frac{x^{4}}{12}+\cdots .
$$

Nesse caso, temos

$$
Z=\int \mathcal{D} \phi \exp \left[-\int \frac{d^{d} x}{a^{d}}\left(J(1-8 d J) a^{2} \phi \nabla^{2} \phi+2 d J(1-4 d J) \phi^{2}+\frac{64}{3}(J d)^{4} \phi^{4}+\cdots\right)\right] .
$$


Os pontos representam termos envolvendo derivadas de ordem superior, tanto na parte quadrática quanto na parte de interação, além das próprias potências maiores que as mostradas em (5.39). Na linguagem do grupo de renormalização, esses termos envolvem apenas operadores irrelevantes que efetivamente não influenciam o limite de baixas energias, inerente ao estudo de transições de fase.

Há um ponto especial nessa expressão que pode ser identificado como o ponto crítico, que ocorre quando o coeficiente de $\phi^{2}$ muda de sinal. Isso está de acordo com a fenomenologia de Landau e define uma temperatura crítica

$$
1-4 d J \equiv \frac{T-T_{c}}{T_{c}}
$$

Lembre que nossa definição de $J$ envolve o fator $\beta$, ou seja, na verdade devemos fazer $J \rightarrow \beta J$. Daí, obtemos uma temperatura crítica $T_{c}=4 d J$. É interessante observar que essa expressão não reproduz (5.31) para o caso $d=2$. Na verdade esse resultado coincide com a temperatura crítica do modelo de Ising na aproximação de campo médio. Quando falamos da equivalência entre o modelo de Ising e um modelo da teoria de campos, estamos nos referindo a uma equivalência entre as quantidades universais, que independem das propriedades microscópicas, por exemplo, os expoentes críticos. O valor da temperatura crítica não é uma quantidade universal.

Prosseguindo com (5.40), fazemos uma expansão em torno do ponto crítico, tal que o coeficiente do termo cinético pode ser aproximado por $1-8 d J \approx-4 d J+O\left(T-T_{c}\right)$. Com isso,

$$
Z=\int \mathcal{D} \phi \exp \left[-\int \frac{d^{d} x}{a^{d}}\left(-4 d J^{2} a^{2} \phi \nabla^{2} \phi+2 d J \frac{\left(T-T_{c}\right)}{T_{c}} \phi^{2}+\frac{64}{3}(J d)^{4} \phi^{4}+\cdots\right)\right] .
$$

Podemos deixar essa expressão numa forma mais apropriada com o reescalonamento $\phi \rightarrow$ $a^{\frac{d-2}{2}} /\left(4 d J^{2}\right)^{1 / 2} \phi$

$$
Z=\int \mathcal{D} \phi \exp \left[-\int d^{d} x\left(-\phi \nabla^{2} \phi+\frac{1}{2 J} \frac{\left(T-T_{c}\right)}{T_{c}} a^{-2} \phi^{2}+\frac{4}{3} d^{2} a^{d-4} \phi^{4}+\cdots\right)\right] .
$$

Essa expressão é identificada como o funcional gerador de uma teoria escalar com interação $\phi^{4}$ definida num espaço euclidiano $d$-dimensional. Desse modo, a discussão anterior mostra que o modelo de Ising, nas proximidades do ponto crítico, pode ser descrito efetivamente por uma teoria de campos. Na verdade, essa característica é mais geral e existem diversos modelos que possuem uma descrição em termos de uma teoria de campos [56]. 


\subsection{Modelo Esférico Clássico}

Devido às dificuldades para obter resultados exatos a partir do modelo de Ising em dimensões mais altas, em 1952 Berlin e Kac [21] introduziram o modelo esférico, que é uma espécie de aproximação contínua do modelo de Ising, definido como

$$
\mathcal{H}=-J \sum_{<\mathbf{r}, \mathbf{r}^{\prime}>} S_{\mathbf{r}} S_{\mathbf{r}^{\prime}}-h \sum_{\mathbf{r}} S_{\mathbf{r}}
$$

que tem a mesma forma da hamiltoniana de Ising, porém as variáveis de spin $S_{\mathbf{r}}$ são contínuas, $-\infty<S_{\mathbf{r}}<\infty$, e sujeitas ao vínculo esférico

$$
\sum_{\mathbf{r}} S_{\mathbf{r}}^{2}=N
$$

Por uma questão de conveniência, estamos usando a notação vetorial $\mathbf{r}$ para os sítios. O vínculo esférico pode ser interpretado como uma lembrança do modelo de Ising no sentido que as variáveis de Ising também o satisfazem. É instrutivo considerar um caso simples para ilustrar a conexão entre as variáveis de Ising e do modelo esférico, tal como na figura (5.2).

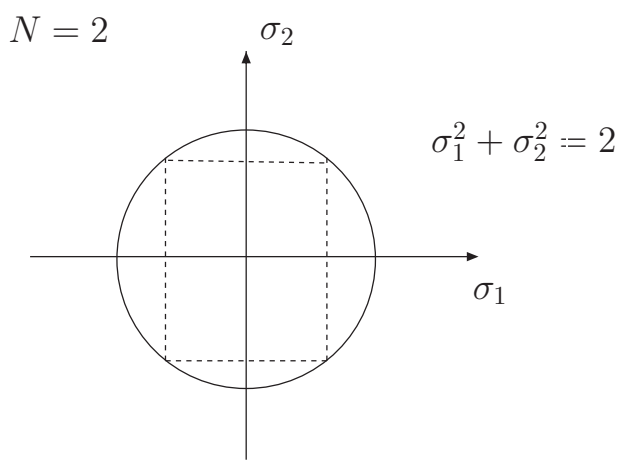

Figura 5.2: Relação entre as configurações do modelo de Ising e do modelo esférico para $N=2$. As variáveis $\sigma_{1,2}$ designam genericamente variáveis de spin (discretas ou contínuas). Assim, as configurações do modelo de Ising estão sobre os vértices do quadrado enquanto que as do modelo esférico estão sobre a circunferência.

A função de partição é definida por

$$
Z=\int \prod_{\mathbf{r}} d S_{\mathbf{r}} \delta\left(\sum_{\mathbf{r}} S_{\mathbf{r}}^{2}-N\right) e^{-\beta \mathcal{H}} .
$$

A maneira usual de calcular essa função de partição é por meio do método do ponto de sela, que pode ser esboçado da seguinte maneira. Usamos uma representação integral 
para a função delta,

$$
\delta\left(\sum_{\mathbf{r}} S_{\mathbf{r}}^{2}-N\right)=\int \frac{d \mu}{2 \pi} e^{i \mu\left(\sum_{\mathbf{r}} S_{\mathbf{r}}^{2}-N\right)} .
$$

Com isso, podemos calcular as integrais gaussianas sobre as variáveis de spin e então a integral em $\mu$ é feita pelo método do ponto de sela no limite termodinâmico $N \rightarrow \infty$. Essa abordagem será adotada no estudo do modelo esférico quântico, no próximo capítulo. Aqui, seguiremos outro método, correspondente ao chamado modelo esférico médio, que de certa forma consiste numa mudança de ensembles. A palavra médio refere-se ao fato que ao invés do vínculo esférico estrito (5.45), consideramos o vínculo em termos de uma média térmica

$$
\sum_{\mathbf{r}}\left\langle S_{\mathbf{r}}^{2}\right\rangle=N
$$

o que não deve conduzir a diferenças nas quantidades termodinâmicas quando comparadas com aquelas obtidas na formulação com (5.45). Uma discussão sobre as propriedades e possíveis diferenças entre o modelo esférico e o modelo esférico médio é feita em [57]. Esse modelo é definido pela função de partição

$$
Z_{M}=\int \prod_{\mathbf{r}} d S_{\mathbf{r}} \exp \left[-\frac{\beta}{2} \sum_{\mathbf{r}, \mathbf{r}^{\prime}} J_{\mathbf{r}, \mathbf{r}^{\prime}} S_{\mathbf{r}} S_{\mathbf{r}^{\prime}}+\beta h \sum_{\mathbf{r}} S_{\mathbf{r}}-\beta \mu \sum_{\mathbf{r}} S_{\mathbf{r}}^{2}\right]
$$

em que o multiplicador de Lagrange $\mu$ pode ser interpretado como um potencial químico. Estamos assumindo que a energia de interação $J_{\mathbf{r}, \mathbf{r}^{\prime}}$ depende somente da distância entre os sítios, podendo ser de curto ou longo alcance. Para o caso usual de interações ferromagnéticas de primeiros vizinhos, $J_{\mathbf{r}, \mathbf{r}^{\prime}}=-J \delta_{\mathbf{r} \pm \mathbf{a}, \mathbf{r}^{\prime}}$. O vínculo esférico médio é implementado de acordo com

$$
\sum_{\mathbf{r}}\left\langle S_{\mathbf{r}}^{2}\right\rangle=-\frac{1}{\beta} \frac{\partial}{\partial \mu} \ln Z_{M}=N
$$

A função de partição é essencialmente um produto de integrais gaussianas e pode ser calculada diretamente sem a necessidade do método do ponto de sela. Para isso é conveniente passar para o espaço de Fourier

$$
S_{\mathbf{r}}=\frac{1}{\sqrt{N}} \sum_{\mathbf{q}} e^{i \mathbf{q} \cdot \mathbf{r}} S_{\mathbf{q}}
$$

com o vetor q pertencente à primeira zona de Brillouin. Com isso, a função de partição torna-se

$$
Z_{M}=\int \prod_{\mathbf{q}} d S_{\mathbf{q}} \exp \left[-\frac{\beta}{2} \sum_{\mathbf{q}} J(\mathbf{q}) S_{\mathbf{q}} S_{-\mathbf{q}}+N^{1 / 2} \beta h S_{\mathbf{0}}-\beta \mu \sum_{\mathbf{q}} S_{\mathbf{q}} S_{-\mathbf{q}}\right],
$$


com

$$
J(\mathbf{q})=\sum_{\mathbf{h}} J(|\mathbf{h}|) e^{i \mathbf{q} \cdot \mathbf{h}}, \quad \operatorname{com} \quad \mathbf{h}=\mathbf{r}-\mathbf{r}^{\prime} .
$$

A condição de realidade da variável $S_{\mathbf{r}}$ implica $S_{-\mathbf{q}}=S_{\mathbf{q}}^{\dagger}$. Assim, $S_{\mathbf{q}} S_{-\mathbf{q}}=S_{\mathbf{q}} S_{\mathbf{q}}^{\dagger}=\left|S_{\mathbf{q}}\right|^{2}$, o que sugere o uso da decomposição de $S_{\mathbf{q}}$ em partes real e imaginária,

$$
S_{\mathbf{q}}=\frac{1}{\sqrt{2}}\left(\operatorname{Re} S_{\mathbf{q}}+i \operatorname{Im} S_{\mathbf{q}}\right) \quad \text { para } \quad \mathbf{q} \neq 0
$$

Para o modo zero mantemos o próprio $S_{0}$. A função de partição é reescrita como

$$
\begin{aligned}
Z_{M} & =\int \prod_{\mathbf{q}>0}\left[d \operatorname{Re} S_{\mathbf{q}}\right]\left[d \operatorname{Im} S_{\mathbf{q}}\right]\left[d S_{0}\right] \exp \left[-\beta \sum_{\mathbf{q}>0}\left(\frac{J(\mathbf{q})}{2}+\mu\right)\left[\left(\operatorname{Re} S_{\mathbf{q}}\right)^{2}+\left(\operatorname{Im} S_{\mathbf{q}}\right)^{2}\right]\right] \\
& \times \exp \left[-\beta\left(\frac{J(0)}{2}+\mu\right) S_{0}^{2}+N^{1 / 2} \beta h S_{0}\right]
\end{aligned}
$$

em que usamos $\operatorname{Re} S_{-\mathbf{q}}=\operatorname{Re} S_{\mathbf{q}}, \operatorname{Im} S_{-\mathbf{q}}=-\operatorname{Im} S_{\mathbf{q}}$ e $J(\mathbf{q})=J(-\mathbf{q})$ para restringir a soma apenas sobre os valores positivos de q. Dessa forma, podemos escrever imediatamente o resultado das integrais gaussianas

$$
Z_{M}=\prod_{\mathbf{q}}\left[\frac{\pi}{\beta\left(\frac{J(\mathbf{q})}{2}+\mu\right)}\right]^{\frac{1}{2}} \exp \left[\frac{N \beta h^{2}}{4\left(\frac{J(0)}{2}+\mu\right)}\right] .
$$

A conexão com a termodinâmica é feita por meio da energia livre $f=-\frac{1}{N \beta} \ln Z_{M}$, fornecendo

$$
f=-\frac{1}{2 N \beta} \sum_{\mathbf{q}} \ln \left[\frac{\pi}{\beta\left(\frac{J(\mathbf{q})}{2}+\mu\right)}\right]-\frac{h^{2}}{4\left(\frac{J(0)}{2}+\mu\right)} .
$$

Finalmente, a imposição do vínculo esférico (5.50) conduz a

$$
\beta=\frac{1}{2 N} \sum_{\mathbf{q}} \frac{1}{\left(\frac{J(\mathbf{q})}{2}+\mu\right)}+\frac{\beta h^{2}}{4\left(\frac{J(0)}{2}+\mu\right)^{2}} .
$$

A partir da energia livre (5.57) juntamente com a expressão do vínculo acima é possível determinar as quantidades termodinâmicas e o comportamento crítico do sistema. No que segue, queremos apenas ilustrar um problema referente ao comportamento da entropia a baixas temperaturas e que constitui também uma das motivações para considerar a versão quântica do modelo. Por uma questão de simplicidade, vamos considerar uma aproximação de campo médio que já é suficiente para nossas propostas. Nessa aproximação, conhecida também como Curie-Weiss, a hipótese básica é que a interação entre 
os vizinhos mais próximos é substituída por uma interação efetiva constante (fraca) entre todos os spins da rede

$$
J_{\mathbf{r}, \mathbf{r}^{\prime}}=-\frac{J}{N} \quad \Rightarrow \quad J(\mathbf{q})=-J \delta_{\mathbf{q}, 0}
$$

Com essa interação a equação do vínculo (5.58) $(\operatorname{com} h=0)$ no limite termodinâmico se reduz a

$$
\beta=\frac{1}{2 \mu}+\frac{1}{2 N} \frac{1}{\left(\mu-\frac{J}{2}\right)} .
$$

Observe que o produto $N\left(\mu-\frac{J}{2}\right)$ no denominador pode gerar uma indeterminação no limite $N \rightarrow \infty$ ao mesmo tempo que $\mu \rightarrow J / 2$ e por isso deve ser mantido. A solução para $\mu$ é

$$
\mu=\left\{\begin{array}{cc}
J / 2, & \text { se } \beta>1 / J \\
1 / 2 \beta, & \text { se } \beta<1 / J
\end{array},\right.
$$

o que define a temperatura crítica $\beta_{c}=1 / J \Rightarrow T_{c}=J$. A energia livre de campo médio no limite $N \rightarrow \infty$ torna-se

$$
f=-\frac{1}{2 N \beta} \ln \left[\frac{\pi}{\beta\left(\mu-\frac{J}{2}\right)}\right]-\frac{1}{2 \beta} \ln \left(\frac{\pi}{\beta \mu}\right) .
$$

A entropia pode ser imediatamente calculada de acordo com $s=-\frac{\partial f}{\partial T}$, que será então uma função de $\beta$ e $\mu$. Mas a equação do vínculo define $\mu$ como uma função de $\beta$. Assim, podemos eliminar $\mu$ em favor da temperatura de acordo com (5.61). Lembre que estamos interessados no comportamento a baixas temperaturas tal que devemos usar o valor de $\mu$ para $T<T_{c}$. O resultado final é

$$
s=\frac{1}{2}\left[\ln \left(\frac{2 \pi T}{T_{c}}\right)+1\right],
$$

mostrando que a entropia diverge quando $T \rightarrow 0$, imcompatível com a terceira lei da termodinâmica. Uma proposta para tratar esse problema é por meio da introdução de flutuações quânticas, ausentes no caso clássico. Isso naturalmente requer a quantização do modelo em questão e de fato corrige essa patologia [58]. Essa foi uma das primeiras motivações para o estudo do modelo esférico quântico, que será discutido em detalhe no próximo capítulo. 


\section{Capítulo 6}

\section{Modelo Esférico Quântico}

Como discutido no capítulo anterior, o modelo esférico clássico apresenta uma patologia na entropia em baixas temperaturas e a quantização do mesmo corrige esse problema. Uma outra motivação para a quantização, talvez a principal, trata-se do próprio estudo das transições de fase quânticas, que tem sido assunto de grande atividade recente [25]. O objetivo deste capítulo é discutir esses aspectos a partir de uma versão quântica do modelo esférico [59].

\subsection{Transições de Fase Quânticas}

Consideremos um sistema quântico que apresente uma transição de fase a uma certa temperatura finita $T$. Presentes no sistema, temos essencialmente flutuações de origem térmica, $k_{B} T$, e de origem quântica, $\hbar \omega$, com $\omega$ alguma frequência característica. O comportamento crítico será determinado por uma competição entre esses dois tipos de flutuações. Em escalas macroscópicas, geralmente as flutuações térmicas predominam sobre as flutuações quânticas, $k_{B} T \gg \hbar \omega$, tal que o comportamento crítico se enquadra na análise clássica de expoentes críticos. Por isso denominaremos as transições a temperatura finita como transições de fase clássicas, ainda que as propriedades do sistema sejam determinadas pela mecânica quântica. Esse é o caso, por exemplo, da transição de fase supercondutora no mercúrio em $T=4,2 K$.

A situação é diferente quando $T=0$, em que as flutuações são exclusivamente de origem quântica. Uma transição de fase que ocorre estritamente à temperatura nula, quando algum parâmetro não-térmico como pressão, campo magnético ou acoplamento, sofre uma variação é denominada de transição de fase quântica. Nesse caso, a análise clássica dos expoentes críticos não é mais válida e o sistema apresenta um comportamento crítico distinto, caracterizado por novos expoentes. Todos esses aspectos são bem ilustrados no modelo esférico quântico. 


\subsection{Modelo Esférico Quântico}

Discutiremos a quantização do modelo esférico por meio da integração funcional. Além de incorporar naturalmente o vínculo esférico estrito, essa formulação deixa bastante evidente a conexão com o modelo $\sigma$ não-linear, a qual será discutida no final.

O modelo esférico clássico foi definido no capítulo anterior, mas o reintroduziremos aqui por conveniência. A hamiltoniana clássica do modelo esférico é dada por

$$
\mathcal{H}_{c}=\frac{1}{2} \sum_{\mathbf{r}, \mathbf{r}^{\prime}} J_{\mathbf{r}, \mathbf{r}^{\prime}} S_{\mathbf{r}} S_{\mathbf{r}^{\prime}}+h \sum_{\mathbf{r}} S_{\mathbf{r}},
$$

em que $\mathbf{r}$ e $\mathbf{r}^{\prime}$ são os vetores da rede, $\left\{S_{\mathbf{r}}\right\}$ é o conjunto de variáveis de spin que assumem valores contínuos, $-\infty<S_{\mathbf{r}}<\infty$, em uma rede hipercúbica $D$-dimensional; $J_{\mathbf{r}, \mathbf{r}^{\prime}}$ é a energia de interação que depende apenas da distância entre os sítios $\mathbf{r}$ e $\mathbf{r}^{\prime}, J_{\mathbf{r}, \mathbf{r}^{\prime}} \equiv$ $J\left(\left|\mathbf{r}-\mathbf{r}^{\prime}\right|\right)$, e $h$ é um campo externo. As variáveis $S_{\mathbf{r}}$ estão sujeitas ao vínculo esférico

$$
\sum_{\mathbf{r}} S_{\mathbf{r}}^{2}=N
$$

com $N$ o número total de sítios da rede. Por outro lado, o vínculo esférico médio é definido como $\sum_{r}\left\langle S_{\mathbf{r}}^{2}\right\rangle=N$, em que $\langle\cdots\rangle$ designa uma média térmica. Como já mencionamos, esperamos que no limite $N \rightarrow \infty$, esses dois vínculos conduzam aos mesmos resultados para as quantidades termodinâmicas.

O primeiro passo para a quantização é proporcionar uma dinâmica ao sistema. Isso pode ser feito adicionando à hamiltoniana um termo cinético envolvendo os momentos conjugados a $S_{\mathbf{r}}$, denotados por $P_{\mathbf{r}}$. No contexto da quantização canônica, $S_{\mathbf{r}}$ e $P_{\mathbf{r}}$ são promovidos a operadores satisfazendo as regras usuais de comutação,

$$
\left[S_{\mathbf{r}}, S_{\mathbf{r}^{\prime}}\right]=0, \quad\left[P_{\mathbf{r}}, P_{\mathbf{r}^{\prime}}\right]=0 \quad \text { and } \quad\left[S_{\mathbf{r}}, P_{\mathbf{r}^{\prime}}\right]=i \delta_{\mathbf{r}, \mathbf{r}^{\prime}}
$$

Com a inclusão de um termo cinético quadrático nos momentos, a hamiltoniana torna-se

$$
\mathcal{H}=\frac{1}{2} g \sum_{\mathbf{r}} P_{\mathbf{r}}^{2}+\frac{1}{2} \sum_{\mathbf{r}, \mathbf{r}^{\prime}} J_{\mathbf{r}, \mathbf{r}^{\prime}} S_{\mathbf{r}} S_{\mathbf{r}^{\prime}}+h \sum_{\mathbf{r}} S_{\mathbf{r}},
$$

em que $g$ é o acoplamento quântico e o limite $g \rightarrow 0$ corresponde ao regime clássico. Para proceder com a quantização canônica é mais simples considerar o modelo esférico médio, em que adicionamos à hamiltoniana um multiplicador de Lagrange tal como em (5.49), usado posteriormente para implementar o vínculo como uma média térmica. A tarefa remanescente é diagonalizar uma hamiltoniana tipo oscilador harmônico, que pode ser feita via transformações de Bogoliubov [60]. 
Procederemos aqui com a quantização via integração funcional. Para essa formulação, precisamos da lagrangiana do modelo que pode ser obtida sem dificuldades a partir de uma transformada de Legendre,

$$
\mathcal{L}=\frac{1}{2 g} \sum_{\mathbf{r}} \dot{S}_{\mathbf{r}}^{2}-\frac{1}{2} \sum_{\mathbf{r}, \mathbf{r}^{\prime}} J_{\mathbf{r}, \mathbf{r}^{\prime}} S_{\mathbf{r}} S_{\mathbf{r}^{\prime}}-h \sum_{\mathbf{r}} S_{\mathbf{r}} .
$$

O ponto significa derivada em relação ao tempo. Por simplicidade, no restante deste capítulo consideraremos o campo externo igual a zero, $h=0$. A seguir, partiremos para o cálculo da função de partição usando o formalismo do tempo imaginário.

\subsubsection{Formalismo do Tempo Imaginário}

O formalismo do tempo imaginário é apropriado para o estudo de teoria de campos a temperatura finita [61, 62, 63]. O ponto fundamental é a semelhança entre o peso de Boltzmann $e^{-\beta H}$ e o operador de evolução temporal da mecânica quântica $e^{-i H t}$. Isso sugere que ao identificarmos a temperatura como um tempo imaginário, $\beta \equiv i t$, poderemos usar diversos resultados conhecidos da mecânica quântica. Em particular, em um sistema bosônico a amplitude de transição de um estado inicial $|i\rangle$ para um estado final $|f\rangle$ pode ser escrita em termos da integração funcional

$$
\left\langle i\left|e^{-i H t}\right| f\right\rangle=\int_{x(0)=x_{i}}^{x(t)=x_{f}} \mathcal{D} x e^{i \int_{0}^{t} d t^{\prime} \mathcal{L}} .
$$

Por outro lado, a função de partição de um sistema quântico é dada por

$$
Z=\operatorname{Tr} e^{-\beta H}=\sum_{n}\left\langle n\left|e^{-\beta H}\right| n\right\rangle
$$

em que a soma em $n$ é simbólica, podendo representar inclusive valores contínuos. Queremos usar (6.6) para expressar os termos envolvidos no traço $\left\langle n\left|e^{-\beta H}\right| n\right\rangle$. Primeiramente, fazemos a mudança de variável $\tau \equiv i t^{\prime}$ em (6.6), tal que $\beta=i t$. Em seguida, consideremos a situação em que no instante inicial temos $x(0)=x_{i}$ e depois de um certo tempo $\beta$ o sistema retorna a essa configuração, $x(\beta)=x_{i}$. Com essa escolha, a expressão (6.6) significa que devemos somar sobre as configurações que satisfaçam a condição $x(0)=x(\beta)=x_{i}$. Mas, a soma sobre as configurações na função de partição representada por $n$ significa que ainda devemos somar sobre todos os valores de $x_{i}$. Dessa forma, o efeito final na função de partição será simplesmente uma integral sobre campos periódicos, com período $\beta$. Em outras palavras, as configurações que devem ser levadas em conta na função de partição são aquelas que satisfazem $x(0)=x(\beta)$

$$
Z=\int \mathcal{D} x e^{-\int_{0}^{\beta} \mathcal{L}_{E}}, \quad \operatorname{com} \quad x(0)=x(\beta),
$$


com a lagrangiana euclidiana $\mathcal{L}_{E} \equiv-\mathcal{L}(\tau)$.

Vamos aplicar esse método para o cálculo da função de partição do modelo esférico quântico. Primeiramente, devemos passar para o tempo euclidiano $\tau=i t, \operatorname{com} \tau \in[0, \beta]$. Além disso, as variáveis bosônicas devem satisfazer a condição de periodicidade no tempo imaginário $S_{\mathbf{r}}(0)=S_{\mathbf{r}}(\beta)$, a qual dá origem às frequências de Matsubara $\omega_{n}=2 n \pi / \beta$, com $n \in \mathrm{Z}$. A função de partição é dada por

$$
Z=\int \mathcal{D} S_{\mathbf{r}} \delta\left(\sum_{\mathbf{r}} S_{\mathbf{r}}^{2}-N\right) e^{-\int_{0}^{\beta} d \tau \mathcal{L}_{E}}
$$

com a lagrangiana euclidiana

$$
\mathcal{L}_{E}=\frac{1}{2 g} \sum_{\mathbf{r}}\left(\frac{\partial S_{\mathbf{r}}(\tau)}{\partial \tau}\right)^{2}+\frac{1}{2} \sum_{\mathbf{r}, \mathbf{r}^{\prime}} J_{\mathbf{r}, \mathbf{r}^{\prime}} S_{\mathbf{r}} S_{\mathbf{r}^{\prime}}
$$

A medida de integração $\mathcal{D} S_{\mathbf{r}}$ representa simbolicamente a integração funcional sobre as variáveis de spin associadas a todos os sítios da rede, ou seja, $\mathcal{D} S_{\mathbf{r}} \equiv \prod_{\mathbf{r}} \mathcal{D} S_{\mathbf{r}}$. Empregando uma representação integral (funcional) para a função delta

$$
\delta\left(\sum_{\mathbf{r}} S_{\mathbf{r}}^{2}-N\right)=\int \mathcal{D} \lambda e^{-\int_{0}^{\beta} d \tau \lambda\left(\sum_{\mathbf{r}} S_{\mathbf{r}}^{2}-N\right)},
$$

podemos escrever

$Z=\int \mathcal{D} S_{\mathbf{r}} \mathcal{D} \lambda \exp \left\{-\int_{0}^{\beta} d \tau\left[\frac{1}{2 g} \sum_{\mathbf{r}}\left(\frac{\partial S_{\mathbf{r}}(\tau)}{\partial \tau}\right)^{2}+\frac{1}{2} \sum_{\mathbf{r}, \mathbf{r}^{\prime}} J_{\mathbf{r}, \mathbf{r}^{\prime}} S_{\mathbf{r}} S_{\mathbf{r}^{\prime}}+\lambda \sum_{\mathbf{r}} S_{\mathbf{r}}^{2}-\lambda N\right]\right\}$.

Essa representação é apropriada pois as integrais sobre $S_{\mathbf{r}}$ tornam-se gaussianas e podem ser imediatamente calculadas, tal como em (5.49). Antes de prosseguir, entretanto, é conveniente introduzir a transformada de Fourier de $S_{\mathbf{r}}$

$$
S_{\mathbf{r}}(\tau)=\frac{1}{\sqrt{N}} \sum_{\mathbf{q}} e^{i \mathbf{q} \cdot \mathbf{r}} S_{\mathbf{q}}(\tau)
$$

Com isso, a ação euclidiana adquire uma forma simples e a função de partição fica

$$
Z=\int \mathcal{D} S_{\mathbf{q}} \mathcal{D} \lambda \exp \left\{-\int_{0}^{\beta} d \tau\left[\sum_{\mathbf{q}} S_{\mathbf{q}}\left(-\frac{1}{2 g} \frac{\partial^{2}}{\partial \tau^{2}}+\frac{1}{2} J(\mathbf{q})+\lambda\right) S_{-\mathbf{q}}-\lambda N\right]\right\} .
$$

Note que identificamos $J(\mathbf{q})$ como a transformada de Fourier da energia de interação $J_{\mathbf{r}, \mathbf{r}^{\prime}}$,

$$
J(\mathbf{q})=\sum_{\mathbf{h}} J(|\mathbf{h}|) e^{i \mathbf{q} \cdot \mathbf{h}}, \quad \text { com } \quad \mathbf{h}=\mathbf{r}-\mathbf{r}^{\prime}
$$


Depois de fazer a integração gaussiana e usar a identidade $\operatorname{det} A=e^{\operatorname{Tr} \ln A}$, obtemos

$$
Z=\int \mathcal{D} \lambda e^{-N S_{\text {eff }}}
$$

em que definimos

$$
S_{e f f} \equiv-\int_{0}^{\beta} d \tau \lambda+\frac{1}{2 N} \operatorname{Tr}\left[\sum_{\mathbf{q}} \ln \left(-\frac{1}{2 g} \frac{\partial^{2}}{\partial \tau^{2}}+\frac{1}{2} J(\mathbf{q})+\lambda\right)\right] .
$$

No limite termodinâmico, $N \rightarrow \infty$, podemos usar o método do ponto de sela para determinar a função de partição. A condição de ponto de sela é

$$
\frac{\delta S_{e f f}}{\delta \lambda\left(\tau^{\prime}\right)}=0
$$

Com o auxílio da identidade $\delta \operatorname{Tr} \ln A=\operatorname{Tr} A^{-1} \delta A$, segue que

$$
1-\frac{1}{2 N} \sum_{\mathbf{q}} \int_{0}^{\beta} d \tau\left\langle\tau\left|\left[\frac{1}{\left(-\frac{1}{2 g} \frac{\partial^{2}}{\partial \tau^{2}}+\frac{1}{2} J(\mathbf{q})+\lambda(\tau)\right)} \delta\left(\tau-\tau^{\prime}\right)\right]\right| \tau\right\rangle=0
$$

em que escolhemos a base $|\tau\rangle$ para o cálculo do traço. Vamos considerar que a solução dessa condição é independente do tempo e denotada por $\lambda \equiv \mu$. A seguir, introduzimos a identidade $\sum_{n}|n\rangle\langle n|=1$, com $\langle\tau \mid n\rangle=\frac{1}{\sqrt{\beta}} e^{i \omega_{n} \tau}$. Nessa base o operador $\frac{\partial^{2}}{\partial \tau^{2}}$ é diagonal, ou seja, $\frac{\partial^{2}}{\partial \tau^{2}}|n\rangle=-\omega_{n}^{2}|n\rangle$. As variáveis $\tau$ e $n$ são análogas às variáveis $q$ e $p$ da mecânica quântica. Com essas observações, obtemos

$$
1-\frac{1}{2 N \beta} \sum_{\mathbf{q}} \sum_{n=-\infty}^{\infty} \frac{1}{\frac{\omega_{n}^{2}}{2 g}+\frac{1}{2} J(\mathbf{q})+\mu}=0 .
$$

A soma sobre as frequências de Matsubara pode ser calculada de acordo com

$$
\sum_{n=-\infty}^{\infty} \frac{1}{n^{2}+y^{2}}=\frac{\pi}{y} \operatorname{coth}(\pi y), \quad y>0
$$

que frequentemente aparece em estudos de teoria de campos a temperatura finita. A dedução dessa fórmula é feita no apêndice C. O resultado é

$$
1-\frac{1}{N} \sum_{\mathbf{q}} \frac{g}{2 \omega_{\mathbf{q}}} \operatorname{coth}\left(\frac{\beta \omega_{\mathbf{q}}}{2}\right)=0
$$

com $\omega_{\mathbf{q}}^{2} \equiv 2 g(\mu+J(\mathbf{q}) / 2)$. Esse resultado é exatamente aquele obtido com o vínculo esférico médio [26]. É claro, também podemos escrever a forma final para a função de 
partição, mas a expressão acima é suficiente para determinar as propriedades críticas do modelo à temperatura finita bem como a temperatura nula. A análise do comportamento crítico pode ser feita considerando o sistema próximo ao ponto crítico, com $\mu \rightarrow 0$ e a energia de interação parametrizada como $J(\mathbf{q}) \sim q^{x}, q \equiv|\mathbf{q}|$, para pequenos valores de $q$. O parâmetro $x$ determina o caráter de curto ou longo alcance da interação. Tipicamente, para interações de curto alcance $x=2$. Como veremos logo abaixo, apesar de considerar o modelo quantizado, para qualquer temperatura finita o sistema exibe um comportamento crítico clássico similar àquele obtido com a versão clássica, pois as flutuações térmicas predominam sobre as flutuações quânticas em escalas macroscópicas. À temperatura zero, no entanto, há uma transição de fase quântica exibindo um comportamento distinto.

Em nossa análise do comportamento crítico vamos verificar a ocorrência da transição de fase e determinaremos as dimensões críticas. A partir daí o cálculo dos expoentes críticos segue sem dificuldades, mas não prosseguiremos com ele aqui.

Consideremos em primeiro lugar transições a temperatura finita. Observe que no limite termodinâmico, a soma em (6.22) pode ser convertida numa integral $\frac{1}{N} \sum_{\mathbf{q}} \rightarrow$ $\int d^{D} q$ restrita à primeira zona de Brillouin, isto é, cada uma das componentes $q_{i} \in$ $[-\pi, \pi]$. Apesar de continuar usando o símbolo de soma elas devem ser entendidas como integrais. Uma análise de convergência em (6.22), mostra que uma divergência pode ocorrer exatamente nas proximidades do ponto crítico, com $\mu=0$, na região de valores $q \sim 0$. Nessa região, podemos escrever $\operatorname{coth} x \sim 1 / x$ e vemos que a integral converge para $D>x$, o que define a dimensão crítica inferior $D_{i}=x$. Portanto, teremos uma transição de fase apenas para $D>x$.

A expressão do vínculo (6.22) no ponto crítico torna-se

$$
1-\frac{1}{N} \sum_{\mathbf{q}} \frac{g_{c}}{2 \sqrt{2 g_{c} q^{x} / 2}} \operatorname{coth}\left(\frac{\beta \sqrt{2 g_{c} q^{x} / 2}}{2}\right)=0 .
$$

Podemos fazer a expansão $\operatorname{coth} x=\frac{1}{x}+\frac{x}{3}+\cdots$ em torno dos valores $q \sim 0$. Agora, consideremos (6.22) nas proximadades do ponto crítico, com $\mu$ pequeno mas finito. Nessa situação, também podemos expandir coth. Subtraindo as equações correspondentes às duas situações acima e fazendo essencialmente uma análise dimensional, obtemos

$$
t_{g} \sim\left\{\begin{array}{cc}
\mu^{\frac{D-x}{x}} & (D<2 x) \\
\mu \ln \mu & (D=2 x) \\
\mu & (D>2 x)
\end{array}\right.
$$

em que $t_{g} \equiv\left(g-g_{c}\right) / g_{c}$. A definição mais natural da distância do ponto crítico para uma transição a temperatura finita é $t_{T} \equiv\left(T-T_{c}\right) / T_{c}$ e pode ser usada de maneira equivalente. A razão de se usar $g$ para definir a distância é para facilitar a comparação com o caso de temperatura nula. A relação anterior determina a dimensão crítica superior, $D_{s}=2 x$, acima da qual a transição é do tipo campo médio. Esse resultados concordam com aqueles 
obtidos a partir do modelo esférico clássico, o que está de acordo com nossa discussão de que em uma transição a temperatura finita o sistema exibe um comportamento crítico clássico, pois as flutuações térmicas são mais relevantes que as flutuações quânticas em escalas macroscópicas.

Para o caso de temperatura zero, observamos que $\operatorname{coth} x \rightarrow 1$, quando $x \rightarrow \infty$, tal que a expressão do vínculo (6.22) se reduz a

$$
1-\frac{1}{N} \sum_{\mathbf{q}} \frac{g}{2 \omega_{\mathbf{q}}}=0 .
$$

Uma simples análise mostra que essa integral converge para $D>x / 2$, resultando na dimensão crítica inferior $D_{c}=x / 2$. Procedendo como no caso anterior, segue

$$
t_{g} \sim\left\{\begin{array}{cc}
\mu^{\frac{2 D-x}{2 x}} & \left(D<\frac{3 x}{2}\right) \\
\mu \ln \mu & \left(D=\frac{3 x}{2}\right) \\
\mu & \left(D>\frac{3 x}{2}\right)
\end{array},\right.
$$

sendo que agora $t_{g} \equiv\left(g-g_{c}^{0}\right) / g_{c}^{0}$, com $g_{c}^{0}$ o valor crítico de $g$ à temperatura zero. Daí obtemos a dimensão crítica superior $D_{s}=3 x / 2$. Esses resultados mostram que a versão quantizada do modelo esférico exibe uma transição de fase quântica caracterizada por novos expoentes críticos [26].

\subsection{Equivalência com o modelo $\sigma$ não-linear}

Antes de finalizar esse capítulo, discutiremos outro aspecto interessante que se trata da equivalência entre o modelo esférico com interações de curto alcance $x=2$ e o modelo $\sigma$ não-linear no limite $N \rightarrow \infty$, com $N$ o número de campos escalares, $\varphi_{i}, i=1, \ldots, N$, definido pela lagrangiana

$$
\mathcal{L}=\frac{1}{2} \partial_{\mu} \varphi \partial^{\mu} \varphi
$$

Estamos omitindo o índice interno de soma $i$. Além disso, os campos $\varphi_{i}$ estão sujeitos ao vínculo

$$
\varphi_{i}^{2} \equiv \varphi^{2}=\frac{N}{2 f},
$$

com $f$ sendo a constante de acoplamento. Devido ao vínculo, podemos adicionar um termo de massa $\left(\sim \varphi^{2}=\right.$ constante) na lagrangiana sem alterar o conteúdo físico do modelo,

$$
\mathcal{L}=\frac{1}{2} \partial_{\mu} \varphi \partial^{\mu} \varphi-\frac{m^{2}}{2} \varphi^{2}
$$

O procedimento para determinar sua ação efetiva, no contexto da expansão $1 / N$ segue, em linhas gerais, os mesmos passos feitos para o cálculo da função de partição do modelo 
esférico. O vínculo é implementado por meio de uma função delta, que é escrita em termos de uma integral sobre um campo auxiliar, digamos $\lambda$, que faz o papel de um multiplicador de Lagrange. Com isso, podemos efetuar a integração sobre $\varphi$ e ficamos com uma ação efetiva em termos de $\lambda$. A ação efetiva tem a estrutura de uma expansão em potências de $1 / N$

$$
S_{e f}=N^{1 / 2} S_{1}+N^{0} S_{2}+N^{-1 / 2} S_{3}+\cdots,
$$

com $S_{n}$ sendo a contribuição para ação efetiva da referida ordem na expansão $1 / N$ e $n$ indicando a potência correspondente do campo auxiliar $\lambda$. Para a série fazer sentido devemos ter o anulamento do termo $S_{1}$, associado a uma potência positiva de $N$. Isso conduz à equação de gap, que escrita no espaço euclidiano tem a forma,

$$
\frac{1}{2 f}-\int \frac{d^{D+1} k_{E}}{(2 \pi)^{D+1}} \frac{1}{k_{E}^{2}+m^{2}}=0 .
$$

Essa equação é muito semelhante a equação do vínculo esférico (6.22) no limite termodinâmico. De fato, considerando o sistema a temperatura finita, devemos levar em conta que a integral sobre a componente zero do momento dá lugar a uma somatória discreta sobre as frequências de Matsubara, tal que

$$
\int \frac{d^{D+1} k_{E}}{(2 \pi)^{D+1}} \rightarrow \frac{1}{\beta} \sum_{n} \int \frac{d^{D} k}{(2 \pi)^{D}}
$$

e $k_{0} \rightarrow \omega_{n}$. De acordo com a fórmula (6.21), obtemos

$$
\frac{1}{2 f}-\int \frac{d^{D} k}{(2 \pi)^{D}} \frac{1}{2 \omega_{k}} \operatorname{coth}\left(\frac{\beta \omega_{k}}{2}\right)=0,
$$

$\operatorname{com} \omega_{k} \equiv \sqrt{\mathbf{k}^{2}+m^{2}}$. Com uma redefinição apropriada dos parâmetros $m$ e $f, m \rightarrow \sqrt{2 g} \mu$ e $f \rightarrow 2^{(D-2) / 2} g^{(D+2) / 2}$ e também do momento de integração $\mathbf{k} \rightarrow \sqrt{2 g} \mathbf{k}$, deixamos a relação acima exatamente igual a (6.22) $\operatorname{com} x=2$. Uma observação importante refere-se aos limites de integração, que no caso acima não tem nenhuma restrição, ao passo que em (6.22) está restrita à primeira zona de Brillouin. O fato é que essa equivalência se dá no limite do contínuo, com o espaçamento da rede $a \rightarrow 0$. No caso do modelo esférico, estavamos considerando um espaçamento unitário, tal que ele não aparecia explicitamente. Restaurando sua dependência, a primeira zona de Brillouin, que para uma rede hipercúbia é delimitada por $[-\pi / a, \pi / a]$ para cada componente do momento, se estende até o infinito. O último passo para estabelecer a conexão completa é tomar o limite $N \rightarrow \infty$. Isso significa que na ação efetiva (6.30) o único termo que contribuirá é $S_{2}$. Mas esse é exatamente a aproximação gaussiana para a integração em $\lambda$, equivalente ao cálculo via o método de ponto de sela.

Ao menos qualitativamente, essa equivalência poderia ter sido antecipada simplesmente tomando o limite do contínuo da lagrangiana do modelo esférico (6.5) seguindo a 
discussão feita na seção 1.3 no primeiro capítulo ou diretamente da equação (5.37), em que a interação entre primeiros vizinhos pode ser aproximada por

$$
\sum_{\mathbf{r}, \mathbf{r}^{\prime}} J_{\mathbf{r}, \mathbf{r}^{\prime}} S_{\mathbf{r}} S_{\mathbf{r}^{\prime}} \sim \sum_{\mathbf{r}} J\left[D(S(t, \mathbf{r}))^{2}-\frac{1}{2}(\nabla S(t, \mathbf{r}))^{2}\right] .
$$

Com isso, segue que a lagrangiana do modelo esférico pode ser escrita numa forma semelhante a do modelo $\sigma$ não-linear (6.29)

$$
\mathcal{L} \sim \frac{1}{2 g}\left(\frac{\partial S(t, \mathbf{r})}{\partial t}\right)^{2}+\frac{J}{2}(\nabla S(t, \mathbf{r}))^{2}-J D(S(t, \mathbf{r}))^{2}
$$

a menos de redefinições dos parâmetros envolvidos de modo a combinar as derivadas temporal e espaciais em $\partial_{\mu} S \partial^{\mu} S$, com as variáveis $S(t, \mathbf{r})$ sujeitas ao vínculo esférico que tem a mesma forma que (6.28). 


\section{Capítulo 7}

\section{Modelo Esférico Quântico Supersimétrico}

Este último capítulo é dedicado à extensão supersimétrica do modelo esférico quântico e à análise do seu comportamento crítico a temperatura finita bem como a temperatura zero. Como veremos, esse modelo é bastante rico, exibindo algumas situações interessantes [59].

\subsection{Idéia de Supersimetria}

A propriedade fundamental da supersimetria é a igualdade do status entre bósons e férmions, implicando a existência de uma simetria entre eles, o que proporciona um caráter mais fundamental. Esse tratamento em pé de igualdade se reflete na igualdade entre os números de graus de liberdade bosônicos e fermiônicos em uma teoria supersimétrica. De uma forma esquemática, essa simetria é traduzida na invariância sob transformações

$$
\delta(\text { bóson }) \sim \text { férmion e } \delta(\text { férmion }) \sim \text { bóson, }
$$

que fazem a transmutação entre bósons e férmions.

Em uma teoria de campos, a supersimetria implica que no espectro as partículas sempre ocorram aos pares, ditas parceiras supersimétricas, possuindo a mesma massa. Apesar de não ser observada experimentalmente nas escalas atuais de energia, muitos físicos acreditam que ela possa existir na forma de uma simetria espontaneamente quebrada. Entre as principais razões que nos levam a essa crença destacamos a sua consistência matemática e os bem conhecidos cancelamentos de divergências ultravioletas, melhorando as propriedades de renormalização da teoria, além de dar uma resposta aos problemas da hierarquia das massas e da unificação das constantes de acoplamento efetivas [64]. 
Queremos implementar esses conceitos no estudo do modelo esférico quântico. Uma questão bastante natural nesse contexto é sobre a possibilidade de adicionar variáveis fermiônicas aos sítios da rede, além da variável bosônica $S_{\mathbf{r}}$. Feito de uma maneira apropriada, isso nos levará a uma teoria supersimétrica. É claro, essa é uma supersimetria num sentido efetivo, refletindo simplesmente a presença de graus de liberdade bosônicos e fermiônicos em cada sítio da rede. Podemos considerar essa extensão e investigar as consequências sobre o comportamento crítico do modelo. Como veremos a seguir, a supersimetria tem uma implicação sobre a existência de uma transição de fase quântica.

\subsection{Supersimetria e Transições de Fase Quânticas}

No contexto da mecânica quântica, a supersimetria requer a existência de supercargas $Q$ e $\bar{Q}$, tal que $\{Q, \bar{Q}\}=\mathcal{H}$, em que $\mathcal{H}$ é a hamiltoniana do sistema, e além disso satistazem $[Q, \mathcal{H}]=[\bar{Q}, \mathcal{H}]=0$; essas supercargas levam um estado bosônico em um fermiônico e vice-versa sendo que o estado fundamental é deixado invariante1. Assim, uma teoria supersimétrica é caracterizada por um estado fundamental bosônico $|0\rangle_{B}$ com energia igual a zero, isto é, aniquilado pelas supercargas e pela hamiltoniana

$$
Q|0\rangle_{B}=\bar{Q}|0\rangle_{B}=\mathcal{H}|0\rangle_{B}=0
$$

Por outro lado, se a supersimetria é quebrada, então pelo menos uma das supercargas não aniquila mais o estado fundamental. Ao invés disso, temos um par de estados bosônico e fermiônico degenerados $|0\rangle_{B}$ e $|0\rangle_{F}$, com energia $E_{0}$, tal que

$$
|0\rangle_{F} \equiv \frac{1}{\sqrt{E_{0}}} \bar{Q}|0\rangle_{B} \quad \text { e }|0\rangle_{B} \equiv \frac{1}{\sqrt{E_{0}}} Q|0\rangle_{F}
$$

Como mencionado no capítulo precedente, em contraste com as transições de fase a temperatura finita, governadas pelas flutuações térmicas, uma transição de fase quântica ocorre a temperatura zero devido a flutuações quânticas associadas ao princípio de incerteza de Heisenberg, dando origem a uma energia de ponto zero. Logo, à temperatura zero, uma transição de fase pode ocorrer numa situação em que a supersimetria está quebrada com a energia do estado fundamental $E_{0}$ diferente de zero, a qual é caracterizada por um parâmetro não térmico $g$, que assume o valor $g_{c}$ no ponto crítico. Próximo ao ponto crítico, temos

$$
E_{0} \sim\left|g-g_{c}\right|^{z \nu}
$$

em que $z$ e $\nu$ são os expoentes críticos dinâmico e do comprimento de correlação, respectivamente. Isso sugere que quando a supersimetria não está quebrada o sistema não exibirá

\footnotetext{
${ }^{1}$ Por simplicidade, estamos nos restringindo a teorias com um estado fundamental bosônico e não degenerado.
} 
um comportamento crítico devido ao anulamento da energia do estado fundamental independente dos valores de $g$. Ao contrário, ele poderá exibir um comportamento crítico se a supersimetria estiver quebrada. De uma forma geral, qualquer desbalanceamento entre bósons e férmions leva a uma quebra de supersimetria. Por exemplo, a supersimetria é sempre quebrada a temperatura finita [65, 66].

Uma maneira conveniente para testar a quebra de supersimetria é introduzindo um parâmetro de ordem. A quantidade mais natural para esse fim é a própria energia do estado fundamental, pois se a supersimetria não está quebrada $E_{0}=0$, caso contrário $E_{0} \neq 0$. Entretanto, por razões de origem topológica, o parâmetro de ordem é usualmente definido pelo índice de Witten $\Delta$ [67], que pode ser representado pela diferença entre o número de estados bosônicos com energia zero $N_{B}^{\left(E_{0}=0\right)}$ e o número de estados fermiônicos com energia zero $N_{F}^{\left(E_{0}=0\right)}$, ou seja, $\Delta \equiv N_{B}^{\left(E_{0}=0\right)}-N_{F}^{\left(E_{0}=0\right)}$. Portanto, segue que

$$
\Delta \equiv N_{B}^{\left(E_{0}=0\right)}-N_{F}^{\left(E_{0}=0\right)}=\left\{\begin{array}{cc}
1 & \text { supersimetria não é quebrada } \\
0 & \text { supersimetria quebrada }
\end{array} .\right.
$$

É instrutivo ver como isso funciona para um sistema bastante simples, um oscilador harmônico quântico supersimétrico dado pela hamiltoniana

$$
H=\omega\left(a_{B}^{\dagger} a_{B}+a_{F}^{\dagger} a_{F}\right), \quad \operatorname{com}\left[a_{B}^{\dagger}, a_{B}\right]=1 \quad \text { e }\left\{a_{F}^{\dagger}, a_{F}\right\}=1 .
$$

Vamos considerar esse sistema a temperatura finita e queremos obter o índice de Witten em função de $\beta, \Delta(\beta)$. A razão básica para a quebra da supersimetria à temperatura finita é devido as diferentes distribuições para bósons e férmions

$$
n_{B}=\frac{1}{e^{\beta \omega}-1} \quad \text { e } \quad n_{F}=\frac{1}{e^{\beta \omega}+1} .
$$

A partir dessas quantidades não é difícil construir um objeto com as propriedades requeridas em (7.5). Em primeiro lugar, $\Delta(\beta)$ deve depender de uma razão entre $n_{B}$ e $n_{F}$, pois se essas distribuições fossem iguais a supersimetria não seria quebrada conduzindo a $\Delta(\beta)=1$, o que é compatível com as escolhas $\Delta(\beta)=n_{B} / n_{F}$ ou $\Delta(\beta)=n_{F} / n_{B}$. Observe que apenas em $T=0$ essas distribuições tornam-se de fato iguais e então $\Delta=1$. É razoável supor que $\Delta(\beta)$ seja finito no limite de altas temperaturas, $T \rightarrow \infty$ ou $\beta \rightarrow 0$, o que exclui a possibilidade $n_{B} / n_{F}$. Assim, ficamos com 2

$$
\Delta(\beta)=\frac{n_{F}}{n_{B}}=\frac{e^{\beta \omega}-1}{e^{\beta \omega}+1} .
$$

Note que, no limite $T \rightarrow \infty$, temos $\Delta \rightarrow 0$, o que está de acordo com (7.5). Por outro lado, para qualquer temperatura finita, $\Delta(\beta)$ tem um valor fracionário, o que indica que a representação usada para seu cálculo não é adequada tal que devemos recorrer à sua definição [68].

\footnotetext{
${ }^{2}$ Esse resultado pode ser confirmado por meio do cálculo preciso usando o método thermofields dynamics [68, que é um formalismo de tempo real baseado na construção de um espaço de Hilbert térmico 63, 69.
} 


\subsection{Extensão Supersimétrica}

O ingrediente básico na construção do modelo esférico quântico supersimétricd3 é a introdução de graus de liberdade fermiônicos em cada sítio da rede para balancear os graus bosônicos existentes. Assim, a cada sítio, além da variável bosônica $S_{\mathbf{r}}$, associaremos as variáveis fermiônicas $\psi_{\mathbf{r}}$ e $\bar{\psi}_{\mathbf{r}}\left(\bar{\psi}_{\mathbf{r}} \equiv \psi_{\mathbf{r}}^{\dagger}\right)$, que no caso quântico satisfazem as relações de anti-comutação

$$
\left\{\psi_{\mathbf{r}}, \psi_{\mathbf{r}^{\prime}}\right\}=0, \quad\left\{\bar{\psi}_{\mathbf{r}}, \bar{\psi}_{\mathbf{r}^{\prime}}\right\}=0 \quad \text { and } \quad\left\{\psi_{\mathbf{r}}, \bar{\psi}_{\mathbf{r}^{\prime}}\right\}=\delta_{\mathbf{r}, \mathbf{r}^{\prime}}
$$

Classicamente elas são tratadas como variáveis de Grassmann. A forma natural para a lagrangiana supersimétrica é

$$
\mathcal{L}_{\text {Susy }}=\frac{1}{2 g} \sum_{\mathbf{r}} \dot{S}_{\mathbf{r}}^{2}-\frac{1}{2} \sum_{\mathbf{r}, \mathbf{r}^{\prime}} J_{\mathbf{r}, \mathbf{r}^{\prime}} S_{\mathbf{r}} S_{\mathbf{r}^{\prime}}+\frac{i}{\sqrt{g}} \sum_{\mathbf{r}} \bar{\psi}_{\mathbf{r}} \dot{\psi}_{\mathbf{r}}-\sum_{\mathbf{r}, \mathbf{r}^{\prime}} U_{\mathbf{r}, \mathbf{r}^{\prime}} \bar{\psi}_{\mathbf{r}} \psi_{\mathbf{r}^{\prime}} .
$$

O requisito de invariância sob as transformações de supersimetria implicará relações entre as interações $J_{\mathbf{r}, \mathbf{r}^{\prime}}$ e $U_{\mathbf{r}, \mathbf{r}^{\prime}}$. Estamos supondo que a interação $U_{\mathbf{r}, \mathbf{r}^{\prime}}$ também dependa apenas da distância entre os sítios $\mathbf{r}$ e $\mathbf{r}^{\prime}$. Além disso, o vínculo sobre a variável bosônica levará a outros vínculos envolvendo as variáveis fermiônicas, o que discutiremos em breve.

O primeiro requisito da supersimetria é a igualdade entre os números de graus de liberdade bosônicos e fermiônicos. Vemos que para a variável real $S_{\mathbf{r}}$ temos um grau, enquanto que para a variável complexa $\psi_{\mathbf{r}}$ temos dois graus. Para compatibilizar esse número devemos usar as equações de movimento

$$
\frac{1}{g} \ddot{S}_{\mathbf{r}}+\sum_{\mathbf{r}^{\prime}} J_{\mathbf{r}, \mathbf{r}^{\prime}} S_{\mathbf{r}^{\prime}}=0 \quad \text { e } \quad \frac{i}{\sqrt{g}} \dot{\psi}_{\mathbf{r}}-\sum_{\mathbf{r}^{\prime}} U_{\mathbf{r}, \mathbf{r}^{\prime}} \psi_{\mathbf{r}^{\prime}}=0
$$

A equação para $\bar{\psi}_{\mathbf{r}}$ é simplesmente o complexo conjugado dessa última equação. Fazendo a decomposição em partes real e imaginária $\psi_{\mathbf{r}}=\psi_{\mathbf{r}}^{1}+i \psi_{\mathbf{r}}^{2}$, segue que

$$
\frac{1}{\sqrt{g}} \dot{\psi}_{\mathbf{r}}^{1}=\sum_{\mathbf{r}^{\prime}} U_{\mathbf{r}, \mathbf{r}^{\prime}} \psi_{\mathbf{r}^{\prime}}^{2} \quad \text { e } \quad \frac{1}{\sqrt{g}} \dot{\psi}_{\mathbf{r}}^{2}=-\sum_{\mathbf{r}^{\prime}} U_{\mathbf{r}, \mathbf{r}^{\prime}} \psi_{\mathbf{r}^{\prime}}^{1}
$$

mostrando que efetivamente as partes real e imaginária não são independentes, reduzindo o número de graus de dois para um em cada sítio da rede. Essa situação em que as equações de movimento são necessárias para garantir a igualdade entre os graus de liberdade é denominada supersimetria on-shell.

\footnotetext{
${ }^{3}$ Uma construção diferente do modelo supersimétrico a partir da quantização estocástica do modelo clássico é feita em [70].
} 
Podemos verificar sem dificuldades que o conjunto de transformações

$$
\delta_{\epsilon} S_{\mathbf{r}}=\bar{\psi}_{\mathbf{r}} \epsilon, \quad \delta_{\epsilon} \psi_{\mathbf{r}}=-\frac{i}{\sqrt{g}} \dot{S}_{\mathbf{r}} \epsilon-\sum_{\mathbf{r}^{\prime}} U_{\mathbf{r}, \mathbf{r}^{\prime}} S_{\mathbf{r}^{\prime}} \epsilon \quad \text { e } \quad \delta_{\epsilon} \bar{\psi}_{\mathbf{r}}=0
$$

e

$$
\delta_{\bar{\epsilon}} S_{\mathbf{r}}=\bar{\epsilon} \psi_{\mathbf{r}}, \quad \delta_{\bar{\epsilon}} \psi_{\mathbf{r}}=0 \quad \text { e } \quad \delta_{\bar{\epsilon}} \bar{\psi}_{\mathbf{r}}=\frac{i}{\sqrt{g}} \dot{S}_{\mathbf{r}} \bar{\epsilon}-\sum_{\mathbf{r}^{\prime}} U_{\mathbf{r}, \mathbf{r}^{\prime}} S_{\mathbf{r}^{\prime}} \bar{\epsilon}
$$

deixa a lagrangiana (17.10) invariante, a menos de termos de superfície, desde que

$$
\sum_{\mathbf{s}} U_{\mathbf{r}, \mathbf{s}} U_{\mathbf{s}, \mathbf{r}^{\prime}} \equiv J_{\mathbf{r}, \mathbf{r}^{\prime}}
$$

Essa relação pode ser vista como uma convolução, tal que sua transformada de Fourier é $[U(\mathbf{q})]^{2}=J(\mathbf{q})$. Os parâmetros da transformação $\epsilon$ e $\bar{\epsilon}$ são quantidades anti-comutantes infinitesimais. As equações (7.13) e (7.14) são as transformações de supersimetria, que relacionam os graus de liberdade bosônicos aos fermiônicos.

O próximo passo é investigar a consistência dos vínculos. Mais precisamente, a implicação das transformações de supersimetria sobre o vínculo esférico

$$
\sum_{\mathbf{r}} S_{\mathbf{r}}^{2}=N
$$

Podemos verificar que, sob as tranformações (17.13) e (17.14), somos levados a

$$
\sum_{\mathbf{r}} \bar{\psi}_{\mathbf{r}} S_{\mathbf{r}}=0, \quad \sum_{\mathbf{r}} \psi_{\mathbf{r}} S_{\mathbf{r}}=0 \quad \text { e } \quad \sum_{\mathbf{r}} \bar{\psi}_{\mathbf{r}} \psi_{\mathbf{r}}=-\sum_{\mathbf{r}, \mathbf{r}^{\prime}} U_{\mathbf{r}, \mathbf{r}^{\prime}} S_{\mathbf{r}} S_{\mathbf{r}^{\prime}}
$$

que formam um conjunto fechados mediante o uso das equações de movimento. Em outras palavras, nenhum outro vínculo surge quando usamos as equações de movimento (7.11). Portanto, para ter uma formulação supersimétrica consistente do modelo esférico precisamos dos quatro vínculos acima, que efetivamente introduzem uma interação entre as variáveis bosônicas e fermiônicas. Eles podem ser implementados por meio de quatro multiplicadores de Lagrange, dois de caráter fermiônico e dois de caráter bosônico.

\subsection{Função de Partição}

Vamos empregar o formalismo do tempo imaginário para o cálculo da função de partição, seguindo essencialmente os mesmos passos do modelo esférico bosônico. Temos que levar em conta, entretanto, que o tratamento de férmions a temperatura finita é um 
pouco diferente. Basicamente devido ao caráter anti-comutante, as variáveis fermiônicas devem satisfazer condições anti-periódicas de contorno. De uma maneira geral, temos

$$
S_{\mathbf{r}}(0)=S_{\mathbf{r}}(\beta), \quad \psi_{\mathbf{r}}(0)=-\psi_{\mathbf{r}}(\beta) \quad \text { and } \quad \bar{\psi}_{\mathbf{r}}(0)=-\bar{\psi}_{\mathbf{r}}(\beta)
$$

O reflexo das condições anti-periódicas é o surgimento das frequências de Matsubara fermiônicas $\omega_{n}^{F}=(2 n+1) \pi / \beta$, com $n \in \mathrm{Z}$, em contraste com as frequências bosônicas $\omega_{n}^{B}=2 n \pi / \beta$.

A função de partição é dada pela integração funcional sobre todos os campos presentes na lagrangiana levando em conta os vínculos (7.16) and (7.17):

$Z=\int \mathcal{D} S_{\mathbf{r}} \mathcal{D} \psi_{\mathbf{r}} \mathcal{D} \bar{\psi}_{\mathbf{r}} \delta\left(\sum_{\mathbf{r}} S_{\mathbf{r}}^{2}-N\right) \delta\left(\sum_{\mathbf{r}} \bar{\psi}_{\mathbf{r}} S_{\mathbf{r}}\right) \delta\left(\sum_{\mathbf{r}} \psi_{\mathbf{r}} S_{\mathbf{r}}\right) \delta\left(\sum_{\mathbf{r}} \bar{\psi}_{\mathbf{r}} \psi_{\mathbf{r}}+\sum_{\mathbf{r}, \mathbf{r}^{\prime}} U_{\mathbf{r}, \mathbf{r}^{\prime}} S_{\mathbf{r}} S_{\mathbf{r}^{\prime}}\right) e^{-\int_{0}^{\beta} d \tau \mathcal{L}_{E}}$

em que $\mathcal{L}_{E}$ é a versão euclidiana de (17.10),

$$
\mathcal{L}_{\text {Susy }}=\frac{1}{2 g} \sum_{\mathbf{r}}\left(\frac{\partial S_{\mathbf{r}}}{\partial \tau}\right)^{2}+\frac{1}{2} \sum_{\mathbf{r}, \mathbf{r}^{\prime}} J_{\mathbf{r}, \mathbf{r}^{\prime}} S_{\mathbf{r}} S_{\mathbf{r}^{\prime}}+\frac{1}{\sqrt{g}} \sum_{\mathbf{r}} \bar{\psi}_{\mathbf{r}} \frac{\partial \psi_{\mathbf{r}}}{\partial \tau}+\sum_{\mathbf{r}, \mathbf{r}^{\prime}} U_{\mathbf{r}, \mathbf{r}^{\prime}} \bar{\psi}_{\mathbf{r}} \psi_{\mathbf{r}^{\prime}}
$$

Como antes, é conveniente usar uma representação integral para as funções delta:

$$
\begin{aligned}
\delta\left(\sum_{\mathbf{r}} S_{\mathbf{r}}^{2}-N\right) & =\int \mathcal{D} \lambda e^{-\int_{0}^{\beta} d \tau \lambda\left(\sum_{\mathbf{r}} S_{\mathbf{r}}^{2}-N\right)}, \\
\delta\left(\sum_{\mathbf{r}} \bar{\psi}_{\mathbf{r}} S_{\mathbf{r}}\right) & =\int \mathcal{D} \zeta e^{-\int_{0}^{\beta} d \tau \sum_{\mathbf{r}} \bar{\psi}_{\mathbf{r}} S_{\mathbf{r}} \zeta}, \\
\delta\left(\sum_{\mathbf{r}} \psi_{\mathbf{r}} S_{\mathbf{r}}\right) & =\int \mathcal{D} \bar{\zeta} e^{-\int_{0}^{\beta} d \tau \sum_{\mathbf{r}} \bar{\zeta} \psi_{\mathbf{r}} S_{\mathbf{r}}}
\end{aligned}
$$

$\mathrm{e}$

$$
\delta\left(\sum_{\mathbf{r}} \bar{\psi}_{\mathbf{r}} \psi_{\mathbf{r}}+\sum_{\mathbf{r}, \mathbf{r}^{\prime}} U_{\mathbf{r}, \mathbf{r}^{\prime}} S_{\mathbf{r}} S_{\mathbf{r}^{\prime}}\right)=\int \mathcal{D} \gamma e^{-\int_{0}^{\beta} d \tau \gamma\left(\sum_{\mathbf{r}} \bar{\psi}_{\mathbf{r}} \psi_{\mathbf{r}}+\sum_{\mathbf{r}, \mathbf{r}^{\prime}} U_{\mathbf{r}, \mathbf{r}^{\prime}} S_{\mathbf{r}} S_{\mathbf{r}^{\prime}}\right)} .
$$

Desse modo, as integrações sobre $S_{\mathbf{r}}$ e $\psi_{\mathbf{r}}, \bar{\psi}_{\mathbf{r}}$ tornam-se gaussianas. Inicialmente, vamos nos concentrar na integração sobre $S_{\mathbf{r}}$, considerando a função de partição efetiva

$$
\begin{aligned}
Z_{e f f}^{\varphi} & \equiv \int \mathcal{D} S_{\mathbf{r}} \exp \left\{-\int_{0}^{\beta} d \tau\left[\frac{1}{2 g} \sum_{\mathbf{r}}\left(\frac{\partial S_{\mathbf{r}}(\tau)}{\partial \tau}\right)^{2}+\frac{1}{2} \sum_{\mathbf{r}, \mathbf{r}^{\prime}} J_{\mathbf{r}, \mathbf{r}^{\prime}} S_{\mathbf{r}} S_{\mathbf{r}^{\prime}}\right.\right. \\
& \left.\left.+\lambda \sum_{\mathbf{r}} S_{\mathbf{r}}^{2}+\gamma \sum_{\mathbf{r}, \mathbf{r}^{\prime}} U_{\mathbf{r}, \mathbf{r}^{\prime}} S_{\mathbf{r}} S_{\mathbf{r}^{\prime}}+\sum_{\mathbf{r}} \varphi_{\mathbf{r}} S_{\mathbf{r}}\right]\right\},
\end{aligned}
$$


em que definimos o campo real bosônico $\varphi_{\mathbf{r}} \equiv \bar{\psi}_{\mathbf{r}} \zeta+\bar{\zeta} \psi_{\mathbf{r}}$. Introduzindo a transformada de Fourier dos campos envolvidos e fazendo a integração gaussiana, obtemos

$$
Z_{e f f}^{\varphi}=\exp \left\{-\frac{1}{2} \operatorname{Tr} \sum_{\mathbf{q}} \ln \mathcal{O}_{\mathbf{q}}\right\} \exp \left\{-\frac{1}{2} \int_{0}^{\beta} d \tau \sum_{\mathbf{q}} \bar{\psi}_{\mathbf{q}} \zeta \mathcal{O}_{\mathbf{q}}^{-1} \bar{\zeta} \psi_{\mathbf{q}}\right\}
$$

com

$$
\mathcal{O}_{\mathbf{q}} \equiv-\frac{1}{2 g} \frac{\partial^{2}}{\partial \tau^{2}}+\frac{1}{2} J(\mathbf{q})+\lambda+\gamma U(\mathbf{q})
$$

Para as integrais fermiônicas, definimos outra função de partição efetiva com a ação já escrita no espaço de Fourier

$$
Z_{e f f} \equiv \int \mathcal{D} \bar{\psi}_{\mathbf{q}} \mathcal{D} \psi_{\mathbf{q}} \exp \left\{-\int_{0}^{\beta} d \tau\left[\sum_{\mathbf{q}} \bar{\psi}_{\mathbf{q}}\left(\frac{1}{\sqrt{g}} \frac{\partial}{\partial \tau}+U(\mathbf{q})+\gamma+\frac{1}{2} \zeta \mathcal{O}_{\mathbf{q}}^{-1} \bar{\zeta}\right) \psi_{\mathbf{q}}\right]\right\}
$$

a qual após a integração fornece

$$
Z_{e f f}=\exp \left[\operatorname{Tr} \sum_{\mathbf{q}} \ln \left(-\frac{1}{\sqrt{g}} \frac{\partial}{\partial \tau}-U(\mathbf{q})-\gamma-\frac{1}{2} \zeta \mathcal{O}_{\mathbf{q}}^{-1} \bar{\zeta}\right)\right]
$$

Reunindo todos os resultados, obtemos

$$
Z=\int \mathcal{D} \lambda \mathcal{D} \gamma \mathcal{D} \bar{\zeta} \mathcal{D} \zeta e^{-N S_{\text {eff }}}
$$

com a ação efetiva

$$
\begin{aligned}
S_{e f f} & \equiv-\int_{0}^{\beta} d \tau \lambda+\frac{1}{2 N} \operatorname{Tr} \sum_{\mathbf{q}} \ln \left(-\frac{1}{2 g} \frac{\partial^{2}}{\partial \tau^{2}}+\frac{1}{2} J(\mathbf{q})+\lambda+\gamma U(\mathbf{q})\right) \\
& -\frac{1}{N} \operatorname{Tr} \sum_{\mathbf{q}} \ln \left(-\frac{1}{\sqrt{g}} \frac{\partial}{\partial \tau}-U(\mathbf{q})-\gamma-\frac{1}{2} \zeta \mathcal{O}_{\mathbf{q}}^{-1} \bar{\zeta}\right) .
\end{aligned}
$$

Considerando o limite termodinâmico $N \rightarrow \infty$, podemos aplicar o método de ponto de sela, que nesse caso envolve as quatro condições

$$
\frac{\delta S_{e f f}}{\delta \lambda(\tau)}=\frac{\delta S_{e f f}}{\delta \gamma(\tau)}=\frac{\delta S_{e f f}}{\delta \zeta(\tau)}=\frac{\delta S_{e f f}}{\delta \bar{\zeta}(\tau)}=0
$$

Vamos procurar soluções dessas equações com os parâmetros $\lambda, \gamma, \zeta$ e $\bar{\zeta}$ independentes do tempo. As condições de extremo em relação aos parâmetros fermiônicos $\zeta$ and $\bar{\zeta}$, podem ser imediatamente satisfeitas com a escolha trivial $\zeta=\bar{\zeta}=0$. Ainda temos duas condições. Vamos definir os pontos de sela correspondentes como $\lambda \equiv \mu$ e $\gamma \equiv \alpha$. A 
condição de extremo com respeito a $\lambda$ pode ser trabalhada da mesma maneira que nos conduziu a (6.22):

$$
1-\frac{1}{N} \sum_{\mathbf{q}} \frac{g}{2 \omega_{\mathbf{q}}^{B}} \operatorname{coth}\left(\frac{\beta \omega_{\mathbf{q}}^{B}}{2}\right)=0,
$$

com a frequência bosônica $\left(\omega_{\mathbf{q}}^{B}\right)^{2} \equiv 2 g\left(\mu+\alpha U(\mathbf{q})+\frac{J(\mathbf{q})}{2}\right)$. A última condição fornece

$$
\frac{1}{N} \sum_{\mathbf{q}} \frac{g}{2 \omega_{\mathbf{q}}^{B}} U(\mathbf{q}) \operatorname{coth}\left(\frac{\beta \omega_{\mathbf{q}}^{B}}{2}\right)-\frac{1}{N} \sum_{\mathbf{q}} \frac{g}{2 \omega_{\mathbf{q}}^{F}}(U(\mathbf{q})+\alpha) \tanh \left(\frac{\beta \omega_{\mathbf{q}}^{F}}{2}\right)=0,
$$

em que a frequência fermiônica é $\left(\omega_{\mathbf{q}}^{F}\right)^{2} \equiv 2 g\left(\frac{\alpha^{2}}{2}+\alpha U(\mathbf{q})+\frac{J(\mathbf{q})}{2}\right)$. Para obter esse resultado usamos a identidade

$$
\sum_{n=-\infty}^{\infty} \frac{1}{(2 n+1)^{2}+y^{2}}=\frac{\pi}{2 y} \tanh \left(\frac{\pi y}{2}\right)
$$

(que pode ser obtida a partir de (6.21)), no cálculo do traço da parte fermiônica, a qual envolve uma soma sobre as frequências de Matsubara fermiônicas $\omega_{n}^{F}=(2 n+1) \pi / \beta$, $n \in \mathrm{Z}$. O comportamento crítico do modelo pode ser determinado analisando as condições de ponto de sela nas proximidades do ponto crítico, da mesma maneira que fizemos para o modelo esférico bosônico.

\subsection{Comportamento Crítico}

Para investigar o comportamento crítico devemos considerar o sistema próximo ao ponto crítico, com $\mu \rightarrow 0$ e $\alpha \rightarrow 0$, em que as integrais são dominadas pelas contribuições de pequenos momentos. Tal como no modelo bosônico, podemos parametrizar as interações para valores pequenos de $|\mathbf{q}| \equiv q$ como $J(\mathbf{q}) \sim q^{x}$ e $U(\mathbf{q}) \sim q^{\frac{x}{2}}$, respeitando o requisito da supersimetria $[U(\mathbf{q})]^{2}=J(\mathbf{q})$. Discutiremos primeiramente o caso de temperatura finita e em seguida o de temperatura zero.

\subsubsection{Temperatura Finita}

Como discutido no início deste capítulo, a supersimetria é incompatível com a temperatura, isto é, a supersimetria é sempre quebrada a temperatura finita. Nessa situação, as flutuações térmicas estão presentes e são responsáveis pela transição de fase. No ponto crítico, as equações (7.33) e (7.34) tornam-se

$$
1-\frac{1}{N} \sum_{\mathbf{q}} \frac{g_{c}}{2 \sqrt{g_{c} J(\mathbf{q})}} \operatorname{coth}\left(\frac{\beta \sqrt{g_{c} J(\mathbf{q})}}{2}\right)=0
$$


$\mathrm{e}$

$\frac{1}{N} \sum_{\mathbf{q}} \frac{g_{c}}{2 \sqrt{g_{c} J(\mathbf{q})}} U(\mathbf{q}) \operatorname{coth}\left(\frac{\beta \sqrt{g_{c} J(\mathbf{q})}}{2}\right)-\frac{1}{N} \sum_{\mathbf{q}} \frac{g_{c}}{2 \sqrt{g_{c} J(\mathbf{q})}} U(\mathbf{q}) \tanh \left(\frac{\beta \sqrt{g_{c} J(\mathbf{q})}}{2}\right)=0$

respectivamente. Essas integrais convergem somente se $D>x$, o que define a dimensão crítica inferior $D_{i}=x$.

Podemos determinar o comportamento crítico subtraindo a expressão (7.33) próxima ao ponto crítico de (7.36). Tecnicamente, próximo ao ponto crítico podemos expandir as funções hiperbólicas coth e tanh para pequenos valores do argumento, de acordo com a discussão acima.

No que segue, vamos considerar alguns casos simples que exibem um comportamento crítico interessante: 1. $\alpha=0 \operatorname{com} \mu$ finito próximo ao ponto crítico; 2. $\mu=0 \operatorname{com} \alpha$ finito próximo ao ponto crítico.

$\alpha=0$ e $\mu$ finito

Subtraindo a equação (7.33) (com $\alpha=0$ e $\mu$ pequeno) de (7.36), obtemos o comportamento

$$
t_{g} \sim\left\{\begin{array}{cc}
\mu^{\frac{D-x}{x}} & (D<2 x) \\
\mu \ln \mu & (D=2 x) \\
\mu & (D>2 x)
\end{array}\right.
$$

com $t_{g} \equiv\left(g-g_{c}\right) / g_{c}$. Daí segue que a dimensão crítica superior é dada por $D_{s}=2 x$. Esse é exatamente o mesmo resultado do modelo esférico bosônico discutido no capítulo anterior. Interpretamos esse resultado como o desacoplamento dos graus de liberdade bosônicos e fermiônicos quando o valor do parâmetro do ponto de sela $\gamma$ é zero, $\alpha=0$. Nessa situação, o sistema efetivamente se reduz ao modelo esférico bosônico.

$\mu=0$ e $\alpha$ finito

Para $\mu=0$ e $\alpha$ pequeno, após a subtração da equação (7.33) de (7.36), encontramos

$$
t_{g} \sim\left\{\begin{array}{cl}
\alpha^{\frac{2(D-x)}{x}} & \left(D<\frac{3 x}{2}\right) \\
\alpha \ln \alpha & \left(D=\frac{3 x}{2}\right. \\
\alpha & \left(D>\frac{3 x}{2}\right)
\end{array}\right.
$$

Nesse caso, não temos mais o desacoplamento dos graus de liberdade bosônicos e fermiônicos, uma vez que o vínculo associado efetivamente indroduz uma interação mediada pelo parâmetro $\gamma$, cujo valor do ponto de sela é agora diferente de zero. Consequentemente, 
o modelo exibe um comportamento crítico distinto e uma dimensão crítica superiror $D_{c}=3 x / 2$.

Vale mencionar que, apesar da quebra da supersimetria devido à temperatura, podemos investigar a situação quando as frequências tornam-se iguais, ou seja, quando $\mu=\alpha^{2} / 2$ (no caso de temperatura zero isso corresponde à situação em que a supersimetria não está quebrada). O comportamento obtido com essa escolha é exatamente aquele de (7.39), que pode ser entendido devido à dominância do termo $q^{\frac{x}{2}}$ sobre $q^{x}$ para momentos pequenos, sempre que $\alpha$ é diferente de zero.

\subsubsection{Temperatura Zero}

No caso de temperatura zero, $\beta \rightarrow \infty$, as funções hiperbólicas coth e tanh são iguais a um e as equaçõs (7.33) e (7.34) se reduzem a

$$
1-\frac{1}{N} \sum_{\mathbf{q}} \frac{g}{2 \omega_{\mathbf{q}}^{B}}=0
$$

$\mathrm{e}$

$$
\frac{1}{N} \sum_{\mathbf{q}} \frac{g}{2 \omega_{\mathbf{q}}^{B}} U(\mathbf{q})-\frac{1}{N} \sum_{\mathbf{q}} \frac{g}{2 \omega_{\mathbf{q}}^{F}}(U(\mathbf{q})+\alpha)=0 .
$$

As integrais convergem para $D>x / 2$, o que determina a dimensão crítica inferior no caso quântico $D_{i}=x / 2$. O procedimento para determinar o comportamento crítico é mesmo da temperatura finita. Note que, no ponto crítico $(\mu=\alpha=0)$ a equação (7.41) é identicamente satisfeita.

Caso Supersimétrico: $\mu=\frac{\alpha^{2}}{2}$

A situação em que a supersimetria não está quebrada é caracterizada pela igualdade entre as frequências bosônicas e fermiônicas, $\omega_{\mathbf{q}}^{B}=\omega_{\mathbf{q}}^{F} \equiv \omega_{\mathbf{q}}$, o que é obtido pela escolha $\mu=\frac{\alpha^{2}}{2}$. A consequência dessa escolha é que mesmo fora (próximo) do ponto crítico as equações (7.40) e (7.41) implicam $\alpha=0$ e também $\mu=0$. Esse resultado indica a ausência de comportamento crítico, de acordo com o argumento discutido no início do capítulo. Ele tem uma interpretação simples: as flutuações quânticas bosônicas e fermiônicas necessárias para conduzirem à transição de fase quântica são canceladas entre si quando a supersimetria não está quebrada. 


\section{Supersimetria Quebrada: $\alpha=0$ e $\mu$ finito}

Nesta situação, as frequências não são mais iguais e a supersimetria é quebrada. Procedendo como antes, podemos determinar o comportamento crítico,

$$
t_{g} \sim\left\{\begin{array}{cc}
\mu^{\frac{2 D-x}{2 x}} & \left(D<\frac{3 x}{2}\right) \\
\mu \ln \mu & \left(D=\frac{3 x}{2}\right) \\
\mu & \left(D>\frac{3 x}{2}\right)
\end{array},\right.
$$

em que agora $t_{g} \equiv\left(g-g_{c}^{0}\right) / g_{c}^{0}$, com $g_{c}^{0}$ o valor crítico de $g$ a temperatura zero. A dimensão crítica superior é $D_{s}=3 x / 2$. Pelo mesmo motivo que aquele apresentado na discussão seguinte à equação (7.38), esse resultado também é o mesmo que o obtido com o modelo esférico quântico bosônico.

\section{Supersimetria Quebrada: $\mu=0$ e $\alpha$ finito}

Neste último caso, em que a supersimetria também é quebrada, obtemos o seguinte comportamento,

$$
t_{g} \sim\left\{\begin{array}{cc}
\alpha^{\frac{2 D-x}{x}} & (D<x) \\
\alpha \ln \alpha & (D=x) \\
\alpha & (D>x)
\end{array},\right.
$$

o qual, em virtude do acoplamento entre os graus de liberdade bosônicos e fermiônicos, é diferente de (7.42), revelando a dimensão crítica superior, $D_{s}=x$. 


\section{Considerações Finais}

Ao longo deste trabalho estudamos problemas em teoria de campos e em mecânica estatística, sempre buscando evidenciar as conexões entre ambos. Umas das motivações para as teorias anisotrópicas vem justamente da mecânica estatística, no contexto de transições de fase exibindo um ponto de Lifshitz. Por outro lado, a teoria de campos proporciona um método eficiente para a quantização e o cálculo da função de partição do modelo esférico estrito, além da inspiração para fazer sua extensão supersimétrica.

Uma questão central no estudo de teorias anisotrópicas é sobre a possibilidade da restauração da simetria de Lorentz em limites de baixas energias. A idéia é que essas teorias com derivadas espaciais de ordem superior possam ser consideradas como descrições efetivas em escalas de energia muito elevadas, em que supomos que a simetria de Lorentz deixa de ser uma simetria fundamental, de modo que no limite de baixas energias o sistema passe a exibir uma dinâmica relativística.

É importante ressaltar que a restauração da simetria de Lorentz nesse contexto, se possível, deve ser considerada em um sentido aproximado, uma vez que não podemos eliminar os termos com derivadas de ordem superior pois senão obteríamos uma teoria não renormalizável. O objetivo é buscar regiões onde a contribuição dos operadores que quebram a simetria de Lorentz possa ser desprezada. Uma outra possibilidade, mas bastante improvável, é que a simetria de Lorentz se manifeste como algum ponto especial nas equações do fluxo do grupo de renormalização, por exemplo, pontos fixos.

Com esses propósitos, desenvolvemos e discutimos diversos aspectos envolvidos no procedimento de renormalização em modelos envolvendo campos escalares e espinoriais exibindo anisotropia entre espaço e tempo caracterizada pelo expoente crítico dinâmico z. Para analisar limites de baixas energias dos modelos apresentados, fizemos uma análise do grupo de renormalização, que essencialmente nos diz como é o comportamento dos parâmetros da teoria quando mudamos a escala de energia.

A partir do estudo de alguns modelos podemos fazer algumas observações gerais. Em primeiro lugar, a simetria de Lorentz não se manifesta como um ponto especial do fluxo do grupo de renormalização. Além disso, as teorias devem ser estáveis no infravermelho, pois assim conseguimos acessar limites de energia arbitrariamente baixos dentro do esquema perturbativo. No caso do modelo $\varphi^{3}$, que é estável no ultravioleta, a constante de acoplamento cresce no limite de baixas energias e não obtemos nenhuma conclusão quanto 
à restauração da simetria de Lorentz. Por isso, consideramos o modelo $\varphi^{4}$, que é estável no infravermelho. Apesar das dificuldades técnicas, esse modelo proporciona um cenário mais favorável, pois com uma escolha particular dos valores iniciais dos parâmetros envolvidos, obtemos uma situação em que a simetria de Lorentz se manifesta num limite de baixas energias.

A situação se torna mais delicada para teorias com mais que um campo interagindo, devido à presença de diversos parâmetros, como no modelo de Yukawa. Apesar de ser estável no infravermelho, a simetria de Lorentz (aproximada) requer um ajuste fino entre os parâmetros envolvidos. O modelo de Yukawa nos dá uma boa idéia do tipo de dificuldades presentes no estudo de teorias com diversos campos. Teorias com valores mais altos do expoente crítico $z$ também exigem a presença de mais parâmetros, o que torna a análise mais complexa. Assim, concluímos que esse não é um mecanismo viável nessas situações.

A generalização de métodos existentes para o caso de teorias com anisotropia pode ser bastante útil também em outros contextos, como em aplicações à matéria condensada. Como uma ferramenta para a investigação de simetrias ao nível quântico em teorias anisotrópicas, generalizamos o método dos produtos normais, o que permite uma análise sistemática das identidades de Ward e das possíveis anomalias. Como uma aplicação, estudamos a anomalia associada às transformações de escala anisotrópica no modelo $\varphi^{4}$.

Na parte da mecânica estatística, o estudo sobre as transições de fase quânticas tem sido assunto de intensa atividade, principalmente pela possibilidade cada vez maior de atingir, experimentalmente, valores muito baixos de temperatura. Naturalmente, do ponto de vista teórico, um modelo exatamente solúvel que apresente resultados não triviais tem um papel de destaque, como é o caso do modelo esférico quântico.

Diversos estudos foram realizados na literatura com a versão quântica do modelo esférico médio. Neste trabalho, fizemos uma abordagem usando métodos da teoria de campos, como a integração funcional e o formalismo do tempo imaginário, para a quantização do modelo esférico estrito. A análise é semelhante àquela usada no modelo sigma não-linear, o que evidencia a conexão entre esse último no limite do número de campos tendendo para o infinito e o modelo esférico com interações de curto alcance. Vimos que, no limite termodinâmico, obtemos o mesmo comportamento crítico que a versão quântica do modelo esférico médio, ou seja, no caso de temperatura finita, o modelo tem um comportamento crítico igual ao do modelo clássico, pois as flutuações térmicas são dominantes. No caso de temperatura zero, ele exibe um comportamento crítico distinto (devido à presença exclusiva de flutuações quânticas), caracterizado por novos expoentes críticos.

Como uma extensão natural, construímos uma versão supersimétrica do modelo esférico quântico, adicionando graus de liberdade fermiônicos em cada sítio da rede, para balancear os graus de liberdade bosônicos. Uma propriedade de teorias supersimétricas é o anulamento da energia do estado fundamental, sugerindo que em uma teoria em que a supersimetria não está quebrada o sistema é incapaz de exibir uma transição de fase quântica. 
Nesse contexto, verificamos que o modelo exibe um comportamento crítico sempre que a supersimetria está quebrada, ou por efeito da temperatura, exibindo um comportamento crítico clássico, ou pelo desbalanceamento das frequências bosônica e fermiônica, com um comportamento crítico quântico. Na situação em que a supersimetria não está quebrada, o modelo não exibe uma transição de fase quântica devido ao anulamento da energia do estado fundamental e consequentemente a ausência de flutuações quânticas, de acordo com o esperado. 


\section{Apêndice A}

\section{Grupo de Renormalização - Modelo de Yukawa}

Este apêndice é dedicado à parte técnica envolvida no estudo do grupo de renormalização do modelo de Yukawa. Uma observação importante é sobre do uso da regularização dimensional em teorias com a presença de férmions. Isso pode trazer dificuldades porque as matrizes de Dirac dependem da dimensão, que na regularização dimensional não é fixa 44. Para tratar esse problema, no cálculo das constantes de renormalização estamos usando o esquema de redução dimensional [71, 72], no qual todas as simplificações com as matrizes de Dirac são feitas na dimensão espacial física $d=6$ e após isso as integrais são promovidas a $d=6-\epsilon$. Ambiguidades que podem ainda estar presentes nesse método se manifestam em ordens mais altas e não afetam nossos cálculos de um laço realizados aqui.

\section{A.1 Parte Divergente das Integrais}

Temos essencialmente dois tipos de integrais para serem analisadas. A forma geral do primeiro tipo ocorre quando temos um laço fermiônico, quer dizer, apenas propagadores fermiônicos nas linhas internas. A forma geral é

$$
J(x, y, z) \equiv \int \frac{d k_{0}}{2 \pi} \frac{d^{d} k}{(2 \pi)^{d}} \frac{k_{0}^{x}|\mathbf{k}|^{y}}{\left[k_{0}^{2}-b_{\psi}^{2} \mathbf{k}^{2}-\left(\alpha_{\psi} \mathbf{k}^{2}+M\right)^{2}\right]^{z}},
$$

em que $x, y$ e $z$ são tais que a integral é no máximo quarticamente divergente. Essa integral é da mesma forma que aquela em (2.16) e seu cálculo segue exatamente os mesmos passos. Observe que o fator de $k_{0}^{x}$ não traz dificuldades para a integração em $k_{0}$. O resultado para 
a parte divergente é;

$$
\begin{aligned}
& J(x, y, z)=\frac{1}{i^{z} \Gamma(z)} \frac{\left(1+(-1)^{x}\right)}{2} \frac{1}{2 \pi} \Gamma\left(\frac{x+1}{2}\right) \frac{2 \pi^{\frac{d}{2}}}{(2 \pi)^{d} \Gamma\left(\frac{d}{2}\right)} \frac{1}{4 \alpha_{\psi}^{2}}\left(\alpha_{\psi}^{2}\right)^{-\frac{(d+y)}{4}} \\
\times \quad & {\left[\alpha_{\psi}^{2} \Gamma\left(\frac{d+y}{4}\right)(-1)^{\frac{1}{8}(2-d-y)} e^{\frac{i \pi x}{4}}\left(i M^{2}\right)^{\frac{1}{4}(2+d+2 x+y-4 z)} \Gamma\left(\frac{-2-d-2 x-y+4 z}{4}\right)\right.} \\
+ & i \alpha_{\psi}^{2} \Gamma\left(\frac{d+y}{4}\right) \frac{\left(b_{\psi}^{2}+2 \alpha_{\psi} M\right)^{2}}{8 \alpha_{\psi}^{2}}(d+y)(-1)^{\frac{1}{8}(2-d-y)} e^{\frac{i \pi x}{4}}\left(i M^{2}\right)^{\frac{1}{4}(-2+d+2 x+y-4 z)} \\
\times \quad & \Gamma\left(\frac{2-d-2 x-y+4 z}{4}\right)-i \alpha_{\psi}\left(b_{\psi}^{2}+2 \alpha_{\psi} M\right) \Gamma\left(\frac{d+y+2}{4}\right)(-1)^{\frac{1}{8}(-d-14 x-y)} e^{i 2 \pi x} \\
\times \quad & \left.\left(i M^{2}\right)^{\frac{1}{4}(d+2 x+y-4 z)} \Gamma\left(\frac{-d-2 x-y+4 z}{4}\right)\right]+ \text { termos finitos. }
\end{aligned}
$$

A outra forma ocorre quando há propagadores de diferentes tipos em um mesmo laço. A forma geral é a seguinte:

$$
\begin{aligned}
& H\left(w, y, z_{1}, z_{2}\right) \\
\equiv & \int \frac{d k_{0}}{2 \pi} \frac{d^{d} k}{(2 \pi)^{d}} \frac{k_{0}^{w}|\mathbf{k}|^{y}}{\left[k_{0}^{2}-b_{\psi}^{2} \mathbf{k}^{2}-\left(\alpha_{\psi} \mathbf{k}^{2}+M\right)^{2}\right]^{z_{1}}\left[k_{0}^{2}-b_{\varphi}^{2} \mathbf{k}^{2}-\alpha_{\varphi}^{2}\left(\mathbf{k}^{2}\right)^{2}-m^{2}\right]^{z_{2}}} .
\end{aligned}
$$

Nesse caso, temos um passo adicional a ser dado, que se trata de empregar a fórmula de parametrização de Feynman

$$
\frac{1}{A^{z_{1}} B^{z_{2}}}=\frac{\Gamma\left(z_{1}+z_{2}\right)}{\Gamma\left(z_{1}\right) \Gamma\left(z_{2}\right)} \int_{0}^{1} d x \frac{x^{z_{1}-1}(1-x)^{z_{2}-1}}{[x A+(1-x) B]^{z_{1}+z_{2}}} .
$$

Identificando $A \equiv k_{0}^{2}-b_{\psi}^{2} \mathbf{k}^{2}-\left(\alpha_{\psi} \mathbf{k}^{2}+M\right)^{2}$ e $B \equiv k_{0}^{2}-b_{\varphi}^{2} \mathbf{k}^{2}-\alpha_{\varphi}^{2}\left(\mathbf{k}^{2}\right)^{2}-m^{2}$, temos

$$
\begin{aligned}
H\left(w, y, z_{1}, z_{2}\right) & =\frac{\Gamma\left(z_{1}+z_{2}\right)}{\Gamma\left(z_{1}\right) \Gamma\left(z_{2}\right)} \int_{0}^{1} d x x^{z_{1}-1}(1-x)^{z_{2}-1} \\
& \times \int \frac{d k_{0}}{2 \pi} \frac{d^{d} k}{(2 \pi)^{d}} \frac{k_{0}^{w}|\mathbf{k}|^{y}}{\left[k_{0}^{2}-f(x) \mathbf{k}^{2}-g(x)\left(\mathbf{k}^{2}\right)^{2}-h(x)\right]^{z_{1}+z_{2}}}
\end{aligned}
$$

sendo que definimos as funções

$$
\begin{gathered}
f(x) \equiv\left(b_{\psi}^{2}+2 \alpha_{\psi} M\right) x+b_{\varphi}^{2}(1-x), \\
g(x) \equiv \alpha_{\psi}^{2} x+\alpha_{\varphi}^{2}(1-x)
\end{gathered}
$$

e

$$
h(x) \equiv M^{2} x+m^{2}(1-x)
$$


Observe agora que, a integral nos momentos acima está efetivamente da forma (A.1) e seu cálculo pode ser conduzida de modo semelhante. O resultado é

$$
\begin{aligned}
& H\left(w, y, z_{1}, z_{2}\right)=\frac{1}{i^{z_{1}+z_{2}} \Gamma\left(z_{1}\right) \Gamma\left(z_{2}\right)} \frac{1}{2 \pi} \frac{\left(1+(-1)^{w}\right)}{2}(-i)^{\frac{-(w+1)}{2}} \Gamma\left(\frac{w+1}{2}\right) \frac{\Omega_{d}}{(2 \pi)^{d}} \\
\times & \int_{0}^{1} d x x^{z_{1}-1}(1-x)^{z_{2}-1} \frac{1}{4 g}(i g)^{\frac{-(d+y)}{4}}\left\{g \Gamma ( \frac { d + y } { 4 } ) \left[(i h)^{\frac{1}{4}\left(2+d+2 w+y-4 z_{1}-4 z_{2}\right)}\right.\right. \\
\times & \Gamma\left(\frac{-2-d-2 w-y+4 z_{1}+4 z_{2}}{4}\right)+i(d+y) \frac{f^{2}}{8 g}(i h)^{\frac{1}{4}\left(-2+d+2 w+y-4 z_{1}-4 z_{2}\right)} \\
\times & \left.\Gamma\left(\frac{2-d-2 w-y+4 z_{1}+4 z_{2}}{4}\right)\right]-f(i g)^{\frac{1}{2}} \Gamma\left(\frac{2+d+y}{2}\right)(i h)^{\frac{1}{4}\left(d+2 w+y-4 z_{1}-4 z_{2}\right)} \\
\times & \left.\Gamma\left(\frac{-d-2 w-y+4 z_{1}+4 z_{2}}{4}\right)\right\} .
\end{aligned}
$$

Resta ainda a integração sobre $x$, mas não é possível calcular essa integral de maneira geral, tal que devemos considerar os casos específicos que aparecem. O próximo passo é determinar as partes divergentes da funções de vértice.

\section{A.1.1 Função de 2 pontos escalar}

Os três diagramas mostrados na figura 3.2 contribuem para a função de vértice de dois pontos em ordens mais baixas não-triviais. Entretanto, considerando uma expansão em laços, o diagrama de dois laços acaba sendo de ordem superior, o que é positivo pois sabemos das dificuldades em extrair sua parte divergente. O diagrama de um laço envolvendo apenas linhas escalares foi considerado no capítulo 2, Devemos analisar então apenas o diagrama com linhas internas fermiônicas, cuja expressão é

$$
\begin{gathered}
g^{2} \int \frac{d k_{0}}{2 \pi} \frac{d^{d} k}{(2 \pi)^{d}} \operatorname{Tr} \Gamma^{5} \frac{1}{\gamma^{0} k^{0}-b_{\psi} \gamma \cdot \mathbf{k}-\alpha_{\psi} \mathbf{k}^{2}-M} \Gamma^{5} \\
\times \frac{1}{\gamma^{0}\left(k^{0}+p^{0}\right)-b_{\psi} \gamma \cdot(\mathbf{k}+\mathbf{p})-\alpha_{\psi}(\mathbf{k}+\mathbf{p})^{2}-M} .
\end{gathered}
$$

Note um sinal negativo adicional devido às regras de anti-comutação para formar o laço fermiônico. Para revelar os termos de pólo aplicamos o operador de Taylor de quarta ordem, $t^{4}$, tal como em (2.27). Na sequência, calcularemos cada termo separadamente. 


\section{Derivadas em relação a $p_{i} p_{j} p_{k} p_{l}$}

Vamos considerar inicialmente o termo de quatro derivadas,

$$
\begin{aligned}
& g^{2} \frac{p_{i} p_{j} p_{k} p_{l}}{4 !} \int \frac{d k_{0}}{2 \pi} \frac{d^{d} k}{(2 \pi)^{d}} \operatorname{Tr} \Gamma^{5} \frac{1}{\gamma^{0} k^{0}-b_{\psi} \gamma \cdot \mathbf{k}-\alpha_{\psi} \mathbf{k}^{2}-M} \Gamma^{5} \\
\times & \left.\frac{\partial^{4}}{\partial p_{i} \partial p_{j} \partial p_{k} \partial p_{l}} \frac{1}{\gamma^{0}\left(k^{0}+p^{0}\right)-b_{\psi} \gamma \cdot(\mathbf{k}+\mathbf{p})-\alpha_{\psi}(\mathbf{k}+\mathbf{p})^{2}-M}\right|_{p=0} .
\end{aligned}
$$

Esse cálculo é muito elaborado e algumas manipulações podem ser feitas para simplificálo um pouco. Primeiro, note que podemos escrever essa expressão de uma forma mais simples,

$$
\begin{aligned}
& g^{2} \frac{p_{i} p_{j} p_{k} p_{l}}{4 !} \int \frac{d k_{0}}{2 \pi} \frac{d^{d} k}{(2 \pi)^{d}} \operatorname{Tr} \Gamma^{5} \frac{1}{\gamma^{0} k^{0}-b_{\psi} \gamma \cdot \mathbf{k}-\alpha_{\psi} \mathbf{k}^{2}-M} \Gamma^{5} \\
\times & \frac{\partial^{4}}{\partial k_{i} \partial k_{j} \partial k_{k} \partial k_{l}} \frac{1}{\gamma^{0} k^{0}-b_{\psi} \gamma \cdot \mathbf{k}-\alpha_{\psi} \mathbf{k}^{2}-M} .
\end{aligned}
$$

Em seguida, é conveniente calcular o traço. Lembre que a dimensionalidade das matrizes de Dirac da representação usada é $d+2$, tal que, $\operatorname{Tr} \gamma^{\mu} \gamma^{\nu}=2^{\frac{d+2}{2}} g^{\mu \nu}$. Dessa maneira,

$$
\begin{aligned}
& g^{2} 2^{\frac{d+2}{2}} \frac{p_{i} p_{j} p_{k} p_{l}}{4 !} \int \frac{d k_{0}}{2 \pi} \frac{d^{d} k}{(2 \pi)^{d}}\left[\frac{-k_{0}^{2}}{[\operatorname{den}]} \frac{\partial^{4}}{\partial k_{i} \partial k_{j} \partial k_{k} \partial k_{l}} \frac{1}{[\operatorname{den}]}+\frac{b_{\psi}^{2} k^{n}}{[\operatorname{den}]} \frac{\partial^{4}}{\partial k_{i} \partial k_{j} \partial k_{k} \partial k_{l}} \frac{k^{n}}{[\text { den }]}\right. \\
+ & \left.\frac{\left(\alpha_{\psi} \mathbf{k}^{2}+M\right)}{[\mathrm{den}]} \frac{\partial^{4}}{\partial k_{i} \partial k_{j} \partial k_{k} \partial k_{l}} \frac{\left(\alpha_{\psi} \mathbf{k}^{2}+M\right)}{[\mathrm{den}]}\right],
\end{aligned}
$$

em que definimos den $\equiv k_{0}^{2}-b_{\psi}^{2} \mathbf{k}^{2}-\left(\alpha_{\psi} \mathbf{k}^{2}+M\right)^{2}$. Agora, podemos descartar as contribuições dos termos finitos nessa expressão. Assim,

$$
g^{2} 2^{\frac{d+2}{2}} \frac{p_{i} p_{j} p_{k} p_{l}}{4 !} \int \frac{d k_{0}}{2 \pi} \frac{d^{d} k}{(2 \pi)^{d}}\left[\frac{-k_{0}^{2}}{[\operatorname{den}]} \frac{\partial^{4}}{\partial k_{i} \partial k_{j} \partial k_{k} \partial k_{l}} \frac{1}{[\operatorname{den}]}+\frac{\alpha_{\psi}^{2} \mathbf{k}^{2}}{[\operatorname{den}]} \frac{\partial^{4}}{\partial k_{i} \partial k_{j} \partial k_{k} \partial k_{l}} \frac{\mathbf{k}^{2}}{[\operatorname{den}]}\right]
$$

+ termos finitos.

Depois de um longo cálculo, já levando em conta a simetria rotacional e usando (A.2), chegamos a

$$
\begin{aligned}
& \frac{g^{2}}{4 !}\left(\mathbf{p}^{2}\right)^{2} \frac{32^{4+\frac{d}{2}}}{d(d+2)}\left[a^{10}\left(-8-10 d+d^{2}\right) J(0,16,6)+2 a^{8}\left(28-8 d-d^{2}\right) J(2,12,6)\right. \\
+ & \left.20 a^{6}(2+3 d) J(4,8,6)+2 a^{4}\left(-44-16 d+d^{2}\right) J(6,4,6)-a^{2} d(d+2) J(8,0,6)\right] \\
+ & \text { termos finitos. }
\end{aligned}
$$

Finalmente, calculando o termo de pólo em $d=6$, encontramos

$$
-\frac{i}{128 \pi^{3}} \frac{g^{2}}{\alpha_{\psi}} \frac{1}{d-6}\left(\mathbf{p}^{2}\right)^{2}
$$




\section{Derivadas em relação a $p_{i} p_{j}$}

O termo que necessitamos avaliar é

$$
\begin{aligned}
& g^{2} \frac{p_{i} p_{j}}{2 !} \int \frac{d k_{0}}{2 \pi} \frac{d^{d} k}{(2 \pi)^{d}} \operatorname{Tr} \Gamma^{5} \frac{1}{\gamma^{0} k^{0}-b_{\psi} \gamma \cdot \mathbf{k}-\alpha_{\psi} \mathbf{k}^{2}-M} \Gamma^{5} \\
\times \quad & \frac{\partial^{2}}{\partial k_{i} \partial k_{j}} \frac{1}{\gamma^{0} k^{0}-b_{\psi} \gamma \cdot \mathbf{k}-\alpha_{\psi} \mathbf{k}^{2}-M} .
\end{aligned}
$$

Depois do cálculo do traço, ficamos com

$$
\begin{aligned}
& g^{2} 2^{\frac{d+2}{2}} \frac{p_{i} p_{j}}{2 !} \int \frac{d k_{0}}{2 \pi} \frac{d^{d} k}{(2 \pi)^{d}}\left[\frac{-k_{0}^{2}}{[\operatorname{den}]} \frac{\partial^{2}}{\partial k_{i} \partial k_{j}} \frac{1}{[\operatorname{den}]}+\frac{b_{\psi}^{2} k^{n}}{[\operatorname{den}]} \frac{\partial^{2}}{\partial k_{i} \partial k_{j}} \frac{k^{n}}{[\text { den }]}\right. \\
+ & \left.\frac{\left(\alpha_{\psi} \mathbf{k}^{2}+M\right)}{[\text { den }]} \frac{\partial^{2}}{\partial k_{i} \partial k_{j}} \frac{\left(\alpha_{\psi} \mathbf{k}^{2}+M\right)}{[\text { den }]}\right] .
\end{aligned}
$$

De acordo com (A.2), segue que

$$
\begin{aligned}
& \frac{g^{2}}{2} \mathbf{p}^{2} 2^{\frac{d+2}{2}}\left[\left(\left(-6+\frac{16}{d}\right) \alpha_{\psi}^{4} b_{\psi}^{2}+\left(2+\frac{32}{d}\right) \alpha_{\psi}^{5} M\right) J(0,8,4)\right. \\
+ & \left(-2+\frac{8}{d}\right) \alpha_{\psi}^{6} J(0,10,4)+\left(8 \alpha_{\psi}^{2} b_{\psi}^{2}+12 \alpha_{\psi}^{3} M\right) J(2,4,4)+4 \alpha_{\psi}^{4} J(2,6,4) \\
- & \left.\left(2 b_{\psi}^{2}+2 \alpha_{\psi} M\right) J(4,0,4)-\left(2+\frac{8}{d}\right) \alpha_{\psi}^{2} J(4,2,4)\right]
\end{aligned}
$$

cuja parte divergente é

$$
-i \frac{M}{16 \pi^{3}} \frac{g^{2}}{\alpha_{\psi}^{2}} \frac{1}{d-6} \mathbf{p}^{2} .
$$

\section{Derivadas em relação a $p_{0} p_{0}$}

Seguindo os mesmos passos anteriores, temos

$$
\begin{aligned}
& g^{2} \frac{p_{0}^{2}}{2 !} \int \frac{d k_{0}}{2 \pi} \frac{d^{d} k}{(2 \pi)^{d}} \operatorname{Tr} \Gamma^{5} \frac{1}{\gamma^{0} k^{0}-b_{\psi} \gamma \cdot \mathbf{k}-\alpha_{\psi} \mathbf{k}^{2}-M} \Gamma^{5} \\
\times \quad & \frac{\partial^{2}}{\partial k_{0} \partial k_{0}} \frac{1}{\gamma^{0} k^{0}-b_{\psi} \gamma \cdot \mathbf{k}-\alpha_{\psi} \mathbf{k}^{2}-M} .
\end{aligned}
$$

Após o cálculo do traço,

$$
\begin{aligned}
& g^{2} 2^{\frac{d+2}{2}} \frac{p_{0}^{2}}{2 !} \int \frac{d k_{0}}{2 \pi} \frac{d^{d} k}{(2 \pi)^{d}}\left[-\frac{k_{0}}{[\operatorname{den}]} \frac{\partial^{2}}{\partial k_{0} \partial k_{0}} \frac{k_{0}}{[\operatorname{den}]}+\frac{b_{\psi}^{2} \mathbf{k}^{2}}{[\operatorname{den}]} \frac{\partial^{2}}{\partial k_{0} \partial k_{0}} \frac{1}{[\operatorname{den}]}\right. \\
+ & \left.\frac{\left(\alpha_{\psi} \mathbf{k}^{2}+M\right)^{2}}{[\operatorname{den}]} \frac{\partial^{2}}{\partial k_{0} \partial k_{0}} \frac{1}{[\operatorname{den}]}\right] .
\end{aligned}
$$


Usando (A.2), temos

$$
-\frac{g^{2}}{2} p_{0}^{2} 2^{\frac{d+2}{2}}\left[-8 J(4,0,4)+6 J(2,0,3)+8 \alpha_{\psi}^{2} J(2,4,4)-2 \alpha_{\psi}^{2} J(0,4,3)\right] .
$$

A parte divergente é

$$
-i \frac{1}{32 \pi^{3}} \frac{g^{2}}{\alpha_{\psi}^{3}} \frac{1}{d-6} p_{0}^{2}
$$

\section{Termo sem derivadas}

O último termo que devemos calcular é quando não há derivadas atuando sobre o diagrama, ou seja,

$$
g^{2} \int \frac{d k_{0}}{2 \pi} \frac{d^{d} k}{(2 \pi)^{d}} \operatorname{Tr} \Gamma^{5} \frac{1}{\gamma^{0} k^{0}-b_{\psi} \gamma \cdot \mathbf{k}-\alpha_{\psi} \mathbf{k}^{2}-M} \Gamma^{5} \frac{1}{\gamma^{0} k^{0}-b_{\psi} \gamma \cdot \mathbf{k}-\alpha_{\psi} \mathbf{k}^{2}-M}
$$

Calculando o traço,

$$
g^{2} 2^{\frac{d+2}{2}} \int \frac{d k_{0}}{2 \pi} \frac{d^{d} k}{(2 \pi)^{d}}\left[\frac{-k_{0}^{2}}{[\operatorname{den}]^{2}}+\frac{b_{\psi}^{2} \mathbf{k}^{2}}{[\mathrm{den}]^{2}}+\frac{\left(\alpha_{\psi} \mathbf{k}^{2}+M\right)^{2}}{[\mathrm{den}]^{2}}\right] .
$$

Usando (A.2), segue que

$$
g^{2} 2^{\frac{d+2}{2}}\left[-J(2,0,2)+b_{\psi}^{2} J(0,2,2)+\alpha_{\psi}^{2} J(0,4,2)+2 \alpha_{\psi} M J(0,2,2)+M^{2} J(0,0,2)\right] .
$$

Por fim, a parte divergente é

$$
-i \frac{\left(3 b_{\psi}^{4}+12 \alpha_{\psi} b_{\psi}^{2} M+8 \alpha_{\psi}^{2} M^{2}\right)}{64 \pi^{3}} \frac{g^{2}}{\alpha_{\psi}^{5}} \frac{1}{d-6} .
$$

\section{A.1.2 Função de 2 pontos espinorial}

A contribuição de um laço para a função de vértice de dois pontos do campo espinorial 
envolve apenas o diagrama da figura 3.4, com a seguinte expressão

$$
\begin{aligned}
& -g^{2} \int \frac{d k_{0}}{2 \pi} \frac{d^{d} k}{(2 \pi)^{d}} \Gamma^{5} \frac{1}{\gamma^{0} k^{0}-b_{\psi} \gamma \cdot \mathbf{k}-\alpha_{\psi} \mathbf{k}^{2}-M} \Gamma^{5} \\
& \times \frac{1}{\left(p_{0}-k_{0}\right)^{2}-b_{\varphi}^{2}(\mathbf{p}-\mathbf{k})^{2}-\alpha_{\varphi}^{2}\left[(\mathbf{p}-\mathbf{k})^{2}\right]^{2}-m^{2}} \\
& =-g^{2} \int \frac{d k_{0}}{2 \pi} \frac{d^{d} k}{(2 \pi)^{d}} \frac{-\gamma^{0} k^{0}+b_{\psi} \gamma \cdot \mathbf{k}+\alpha_{\psi} \mathbf{k}^{2}+M}{k_{0}^{2}-b_{\psi}^{2} \mathbf{k}^{2}-\left(\alpha_{\psi} \mathbf{k}^{2}+M\right)^{2}} \\
& \times \frac{1}{\left(p_{0}-k_{0}\right)^{2}-b_{\varphi}^{2}(\mathbf{p}-\mathbf{k})^{2}-\alpha_{\varphi}^{2}\left[(\mathbf{p}-\mathbf{k})^{2}\right]^{2}-m^{2}} .
\end{aligned}
$$

Visto que o grau de divergência superficial é $d(G)=2$, devemos aplicar o operador de Taylor de segunda ordem $t^{2}$. Seja $I(p, k)$ o integrando acima, então

$$
t^{2} I(p, k)=I(0, k)+\left.p_{i} \frac{\partial I(p, k)}{\partial p_{i}}\right|_{p=0}+\left.p_{0} \frac{\partial I(p, k)}{\partial p_{0}}\right|_{p=0}+\left.\frac{p_{i} p_{j}}{2 !} \frac{\partial^{2} I(p, k)}{\partial p_{i} \partial p_{j}}\right|_{p=0} .
$$

Assim como no caso anterior é conveniente separar o cálculo em partes de acordo com os termos do operador acima.

\section{Derivadas em relação a $p_{i} p_{j}$}

O termo com duas derivadas é

$$
-g^{2} \frac{p_{i} p_{j}}{2 !} \int \frac{d k_{0}}{2 \pi} \frac{d^{d} k}{(2 \pi)^{d}} \frac{-\gamma^{0} k^{0}+b_{\psi} \gamma \cdot \mathbf{k}+\alpha_{\psi} \mathbf{k}^{2}+M}{k_{0}^{2}-b_{\psi}^{2} \mathbf{k}^{2}-\left(\alpha_{\psi} \mathbf{k}^{2}+M\right)^{2}} \frac{\partial^{2}}{\partial k_{i} \partial k_{j}} \frac{1}{k_{0}^{2}-b_{\varphi}^{2} \mathbf{k}^{2}-\alpha_{\varphi}^{2}\left(\mathbf{k}^{2}\right)^{2}-m^{2}}
$$

Depois do cálculo das derivadas e mantendo apenas os termos divergentes, obtemos

$$
\begin{aligned}
& -g^{2} \frac{\mathbf{p}^{2}}{2 !} \int \frac{d k_{0}}{2 \pi} \frac{d^{d} k}{(2 \pi)^{d}}\left[\frac{\left(\frac{8 \alpha_{\varphi}^{2}}{d}+4 \alpha_{\varphi}^{2}\right) \alpha_{\psi}\left(\mathbf{k}^{2}\right)^{2}}{\left[k_{0}^{2}-b_{\psi}^{2} \mathbf{k}^{2}-\left(\alpha_{\psi} \mathbf{k}^{2}+M\right)^{2}\right]\left[k_{0}^{2}-b_{\varphi}^{2} \mathbf{k}^{2}-\alpha_{\varphi}^{2}\left(\mathbf{k}^{2}\right)^{2}-m^{2}\right]^{2}}\right. \\
& \left.+\frac{\frac{32 \alpha_{\psi} \alpha_{\varphi}^{4}}{d}\left(\mathbf{k}^{2}\right)^{4}}{\left[k_{0}^{2}-b_{\psi}^{2} \mathbf{k}^{2}-\left(\alpha_{\psi} \mathbf{k}^{2}+M\right)^{2}\right]\left[k_{0}^{2}-b_{\varphi}^{2} \mathbf{k}^{2}-\alpha_{\varphi}^{2}\left(\mathbf{k}^{2}\right)^{2}-m^{2}\right]^{3}}\right]
\end{aligned}
$$

em que, usamos a simetria rotacional para fazer a troca $k_{i} k_{j} \rightarrow \mathbf{k}^{2} \delta_{i j} / d$. De acordo com a função $H\left(w, y, z_{1}, z_{2}\right)$ definida em (A.9), podemos escrever

$$
-g^{2} \frac{\mathbf{p}^{2}}{2}\left[\left(\frac{8 \alpha_{\varphi}^{2}}{d}+4 \alpha_{\varphi}^{2}\right) \alpha_{\psi} H(0,4,1,2)+\frac{32 \alpha_{\psi} \alpha_{\varphi}^{4}}{d} H(0,8,1,3)\right] .
$$

O termo divergente é

$$
-\frac{i}{384 \pi^{3}} \frac{\left(-8 \alpha_{\varphi}^{3} \alpha_{\psi}^{2}+3 \alpha_{\varphi}^{4} \alpha_{\psi}-\alpha_{\psi}^{5}+6 \alpha_{\varphi}^{2} \alpha_{\psi}^{3}\right)}{\alpha_{\varphi}\left(\alpha_{\varphi}^{2}-\alpha_{\psi}^{2}\right)^{3}} g^{2} \frac{1}{d-6} \mathbf{p}^{2} .
$$




\section{Derivada em relação a $p_{0}$}

O termo de derivada de primeira ordem em $p_{0}$ é dado por

$$
g^{2} p_{0} \int \frac{d k_{0}}{2 \pi} \frac{d^{d} k}{(2 \pi)^{d}} \frac{-\gamma^{0} k^{0}+b_{\psi} \gamma \cdot \mathbf{k}+\alpha_{\psi} \mathbf{k}^{2}+M}{k_{0}^{2}-b_{\psi}^{2} \mathbf{k}^{2}-\left(\alpha_{\psi} \mathbf{k}^{2}+M\right)^{2}} \frac{\partial}{\partial k_{0}} \frac{1}{k_{0}^{2}-b_{\varphi}^{2} \mathbf{k}^{2}-\alpha_{\varphi}^{2}\left(\mathbf{k}^{2}\right)^{2}-m^{2}}
$$

Mantendo apenas os termos divergentes,

$$
g^{2} p_{0} \gamma^{0} \int \frac{d k_{0}}{2 \pi} \frac{d^{d} k}{(2 \pi)^{d}} \frac{2 k_{0}^{2}}{\left[k_{0}^{2}-b_{\psi}^{2} \mathbf{k}^{2}-\left(\alpha_{\psi} \mathbf{k}^{2}+M\right)^{2}\right]\left[k_{0}^{2}-b_{\varphi}^{2} \mathbf{k}^{2}-\alpha_{\varphi}^{2}\left(\mathbf{k}^{2}\right)^{2}-m^{2}\right]^{2}},
$$

a qual fornece

$$
2 g^{2} p_{0} \gamma^{0} H(2,0,1,2) \text {. }
$$

O termo de pólo é

$$
\frac{i}{128 \pi^{3}} \frac{2 \alpha_{\psi}-\frac{\alpha_{\varphi}^{2}\left(\alpha_{\varphi}^{2}+\alpha_{\psi}^{2}\right)}{\alpha_{\varphi}^{3}}}{\left(\alpha_{\varphi}^{2}-\alpha_{\psi}^{2}\right)^{2}} g^{2} \frac{1}{d-6} p_{0} \gamma^{0}
$$

\section{Derivada em relação a $p_{i}$}

O termo com uma derivada é

$$
g^{2} p_{i} \int \frac{d k_{0}}{2 \pi} \frac{d^{d} k}{(2 \pi)^{d}} \frac{-\gamma^{0} k^{0}+b_{\psi} \gamma \cdot \mathbf{k}+\alpha_{\psi} \mathbf{k}^{2}+M}{k_{0}^{2}-b_{\psi}^{2} \mathbf{k}^{2}-\left(\alpha_{\psi} \mathbf{k}^{2}+M\right)^{2}} \frac{\partial}{\partial k_{i}} \frac{1}{k_{0}^{2}-b_{\varphi}^{2} \mathbf{k}^{2}-\alpha_{\varphi}^{2}\left(\mathbf{k}^{2}\right)^{2}-m^{2}} .
$$

Levando em conta a simetria rotacional e considerando apenas as partes divergentes, obtemos

$$
g^{2} \mathbf{p} \cdot \gamma \frac{4 \alpha_{\varphi}^{2} b_{\psi}}{d} \int \frac{d k_{0}}{2 \pi} \frac{d^{d} k}{(2 \pi)^{d}} \frac{\left(\mathbf{k}^{2}\right)^{2}}{\left[k_{0}^{2}-b_{\psi}^{2} \mathbf{k}^{2}-\left(\alpha_{\psi} \mathbf{k}^{2}+M\right)^{2}\right]\left[k_{0}^{2}-b_{\varphi}^{2} \mathbf{k}^{2}-\alpha_{\varphi}^{2}\left(\mathbf{k}^{2}\right)^{2}-m^{2}\right]^{2}},
$$

que pode ser identificado como,

$$
\frac{4 \alpha_{\varphi}^{2} b_{\psi}}{d} g^{2} \mathbf{p} \cdot \gamma H(0,4,1,2)
$$

Finalmente, a parte divergente resulta em

$$
\frac{i}{384 \pi^{3}} \frac{\left(2 \alpha_{\varphi}^{3}-3 \alpha_{\varphi}^{2} \alpha_{\psi}+\alpha_{\psi}^{3}\right)}{\alpha_{\varphi} \alpha_{\psi}\left(\alpha_{\varphi}^{2}-\alpha_{\psi}^{2}\right)^{2}} b_{\psi} g^{2} \frac{1}{d-6} \mathbf{p} \cdot \gamma
$$




\section{Termo sem derivadas}

Tomando o momento externo igual a zero (A.29), obtemos

$$
-g^{2} \int \frac{d k_{0}}{2 \pi} \frac{d^{d} k}{(2 \pi)^{d}} \frac{-\gamma^{0} k^{0}+b_{\psi} \gamma \cdot \mathbf{k}+\alpha_{\psi} \mathbf{k}^{2}+M}{k_{0}^{2}-b_{\psi}^{2} \mathbf{k}^{2}-\left(\alpha_{\psi} \mathbf{k}^{2}+M\right)^{2}} \frac{1}{k_{0}^{2}-b_{\varphi}^{2} \mathbf{k}^{2}-\alpha_{\varphi}^{2}\left(\mathbf{k}^{2}\right)^{2}-m^{2}} .
$$

Os termos lineares no momento não contribuem, tal que

$$
-g^{2}\left[\alpha_{\psi} H(0,2,1,1)+M H(0,0,1,1)\right] .
$$

O termo de pólo é

$$
\begin{aligned}
& -i \frac{g^{2}}{256 \pi^{3} \alpha_{\varphi}^{3} \alpha_{\psi}^{2}\left(\alpha_{\varphi}^{2}-\alpha_{\psi}^{2}\right)^{2}} \\
\times & {\left[\alpha_{\psi}^{5} b_{\varphi}^{2}+\alpha_{\varphi}^{2} \alpha_{\psi}^{2}\left[\left(2 \alpha_{\varphi}-3 \alpha_{\psi}\right) b_{\varphi}^{2}+\left(-3 \alpha_{\varphi}+2 \alpha_{\psi}\right) b_{\psi}^{2}+2 \alpha_{\psi}\left(-2 \alpha_{\varphi}+\alpha_{\psi}\right) M\right)\right] } \\
+ & \left.\alpha_{\varphi}^{4}\left(\alpha_{\varphi} b_{\psi}^{2}+2 \alpha_{\psi}^{2} M\right)\right] \frac{1}{d-6} .
\end{aligned}
$$

\section{A.1.3 Função de 3 pontos}

A contribuição de um laço para a função de vértice com duas linhas externas bosônicas e uma fermiônica correspondente ao diagrama da figura 3.5. Como a divergência é logaritmica, basta considerar a expressão com os momentos externos iguais a zero,

$$
\begin{aligned}
& i g^{3} \int \frac{d k_{0}}{2 \pi} \frac{d^{d} k}{(2 \pi)^{d}} \Gamma^{5} \frac{1}{\gamma^{0} k^{0}-b_{\psi} \gamma \cdot \mathbf{k}-\alpha_{\psi} \mathbf{k}^{2}-M} \Gamma^{5} \frac{1}{\gamma^{0} k^{0}-b_{\psi} \gamma \cdot \mathbf{k}-\alpha_{\psi} \mathbf{k}^{2}-M} \Gamma^{5} \\
\times & \frac{1}{k_{0}^{2}-b_{\varphi}^{2} \mathbf{k}^{2}-\alpha_{\varphi}^{2}\left(\mathbf{k}^{2}\right)^{2}-m^{2}} .
\end{aligned}
$$

Com uma manipulação simples das matrizes de Dirac, podemos escrever

$$
\begin{aligned}
& -i g^{3} \Gamma^{5} \int \frac{d k_{0}}{2 \pi} \frac{d^{d} k}{(2 \pi)^{d}} \frac{1}{\left[k_{0}^{2}-b_{\psi}^{2} \mathbf{k}^{2}-\left(\alpha_{\psi} \mathbf{k}^{2}+M\right)^{2}\right]\left[k_{0}^{2}-b_{\varphi}^{2} \mathbf{k}^{2}-\alpha_{\varphi}^{2}\left(\mathbf{k}^{2}\right)^{2}-m^{2}\right]} \\
= & -i g^{3} \Gamma^{5} H(0,0,1,1) .
\end{aligned}
$$

A parte divergente é

$$
-\frac{1}{128 \pi^{3}} \frac{1}{\alpha_{\varphi} \alpha_{\psi}^{2}+\alpha_{\varphi}^{2} \alpha_{\psi}} g^{3} \Gamma^{5} \frac{1}{d-6} .
$$




\section{A.1.4 Função de 4 pontos}

A contribuição de um laço para a função de vértice de quatro pontos envolve os dois diagramas da figura 3.3. A divergência dessa função é logaritmica e basta considerar os gráficos com momento externo nulo. O diagrama contendo apenas linhas escalares foi analisado no capítulo anterior, enquanto que a expressão associada ao primeiro diagrama da figura 3.3 é

$$
\begin{aligned}
& -\quad 6 g^{4} \int \frac{d k_{0}}{2 \pi} \frac{d^{d} k}{(2 \pi)^{d}} \operatorname{Tr} \Gamma^{5} \frac{1}{\gamma^{0} k^{0}-b_{\psi} \gamma \cdot \mathbf{k}-\alpha_{\psi} \mathbf{k}^{2}-M} \Gamma^{5} \frac{1}{\gamma^{0} k^{0}-b_{\psi} \gamma \cdot \mathbf{k}-\alpha_{\psi} \mathbf{k}^{2}-M} \\
& \times \quad \Gamma^{5} \frac{1}{\gamma^{0} k^{0}-b_{\psi} \gamma \cdot \mathbf{k}-\alpha_{\psi} \mathbf{k}^{2}-M} \Gamma^{5} \frac{1}{\gamma^{0} k^{0}-b_{\psi} \gamma \cdot \mathbf{k}-\alpha_{\psi} \mathbf{k}^{2}-M} .
\end{aligned}
$$

Usando a álgebra das matrizes de Dirac e calculando o traço, essa expressão se reduz a

$$
-6 g^{4} 2^{\frac{d+2}{2}} \int \frac{d k_{0}}{2 \pi} \frac{d^{d} k}{(2 \pi)^{d}} \frac{1}{\left[k_{0}^{2}-b_{\psi}^{2} \mathbf{k}^{2}-\left(\alpha_{\psi} \mathbf{k}^{2}+M\right)^{2}\right]^{2}} .
$$

De acordo com (A.1), obtemos

$$
-6 g^{4} 2^{\frac{d+2}{2}} J(0,0,2) .
$$

Finalmente, o termo de pólo é

$$
\frac{3 i}{8 \pi^{3}} \frac{1}{\alpha_{\psi}^{3}} g^{4} \frac{1}{d-6} .
$$

Agora que finalizamos o cálculo da parte divergente de todas as funções de vértice relevantes podemos prosseguir com o grupo de renormalização. O primeiro passo é deduzir a equação do grupo de renormalização para uma teoria com campos bosônicos e fermiônicos.

\section{A.2 Grupo de Renormalização}

A dedução da equação do grupo de renormalização no presente caso é semelhante àquela desenvolvida no segundo capítulo e então podemos seguir o mesmo raciocínio. A função de vértice com $N_{B}$ linhas externas bosônicas amputadas e $N_{F}$ fermiônicas é definida como

$$
\begin{aligned}
& (2 \pi)^{d+1} \delta\left(p_{1}+\cdots+p_{N_{B}}+q_{1}+\cdots+q_{N_{F}}\right) \Gamma_{a_{1} \cdots a_{N_{F}}}^{\left(N_{B}+N_{F}\right)}\left(p_{1}, \cdots, p_{N_{B}}, q_{1}, \cdots, q_{N_{F}}\right) \\
\equiv & \prod_{i=1}^{N_{B}} \Delta^{-1}\left(p_{i}\right) \prod_{j=1}^{N_{F} / 2} S_{a_{j} b_{j}}^{-1}\left(q_{j}\right) \prod_{k=N_{F} / 2+1}^{N_{F}} S_{b_{k} a_{k}}^{-1}\left(q_{k}\right) \int d^{D} x_{1} \cdots d^{D} x_{N_{B}} \int d^{D} y_{1} \cdots d^{D} y_{N_{F}} \\
\times & \left\langle 0\left|T \varphi\left(x_{1}\right) \cdots \varphi\left(x_{N_{B}}\right) \psi_{b_{1}}\left(y_{1}\right) \cdots \psi_{b_{N_{F} / 2}}\left(y_{N_{F} / 2}\right) \bar{\psi}_{b_{N_{F} / 2+1}}\left(y_{b_{N_{F} / 2+1}}\right) \cdots \bar{\psi}_{b_{N_{F}}}\left(y_{b_{N_{F}}}\right)\right| 0\right\rangle^{\text {prop }} \\
\times & e^{i \sum_{i=1}^{N_{B} p_{i} x_{i}+i \sum_{j=1}^{N_{F}} q_{j} y_{j}} .}
\end{aligned}
$$


As letras $a$ e $b$ designam índices espinoriais. A relação entre a função de vértice renormalizada, $\Gamma^{\left(N_{B}+N_{F}\right)}$, e a não-renormalizada, $\Gamma_{n}^{\left(N_{B}+N_{F}\right)}$, é a seguinte

$$
\Gamma_{n}^{\left(N_{B}+N_{F}\right)}=Z_{\varphi}^{-\frac{N_{B}}{2}} Z_{\psi}^{-\frac{N_{F}}{2}} \Gamma^{\left(N_{B}+N_{F}\right)} .
$$

A partir daí é fácil obter a equação do grupo de renormalização;

$$
\left[\mu \frac{\partial}{\partial \mu}+D_{\varphi}+D_{\psi}+\beta_{g} \frac{\partial}{\partial g}+\beta_{\lambda} \frac{\partial}{\partial \lambda}-N_{B} \gamma_{\varphi}-N_{F} \gamma_{\psi}\right] \Gamma^{(N)}(p, m, b, \alpha, \lambda, \mu)=0,
$$

em que definimos os operadores diferenciais

$$
D_{\varphi} \equiv \delta_{m^{2}} \frac{\partial}{\partial m^{2}}+\beta_{b_{\varphi}^{2}} \frac{\partial}{\partial b_{\varphi}^{2}}+\beta_{\alpha_{\varphi}^{2}} \frac{\partial}{\partial \alpha_{\varphi}^{2}}
$$

e

$$
D_{\psi} \equiv \delta_{M} \frac{\partial}{\partial M}+\beta_{b_{\psi}} \frac{\partial}{\partial b_{\psi}}+\beta_{\alpha_{\psi}} \frac{\partial}{\partial \alpha_{\psi}} .
$$

A dependência da função de vértice com os parâmetros está sendo indicada genericamente por $m, b, \alpha, \lambda$. O parâmetro $\mu$ é introduzido da maneira usual por meio de $g \rightarrow g \mu^{\frac{\epsilon}{2}} \mathrm{e}$ $\lambda \rightarrow \lambda \mu^{\epsilon}$. Como os próximos passos já foram ilustrados em detalhes no caso do campo escalar, nas seções seguintes apresentaremos de modo sucinto os resultados para as funções de vértices envolvidas.

\section{A.2.1 Função de 2 pontos escalar}

A função de vértice de dois pontos renormalizada $\left(N_{B}=2\right.$ e $\left.N_{F}=0\right)$, ou seja, depois da aplicação do operador de subtração dos pólos, é dada por

$$
\begin{aligned}
\Gamma_{\varphi}^{(2)}(p) & =i\left[p_{0}^{2}-b_{\varphi}^{2} \mathbf{p}^{2}-\alpha_{\varphi}^{2}\left(\mathbf{p}^{2}\right)^{2}-m^{2}+g^{2}\left(\text { Parte finita }_{1}-\ln \mu \text { Resíduo }_{1}\right)\right. \\
& \left.-i \lambda\left(\text { Parte finita }_{2}-\ln \mu \text { Resíduo }_{2}\right)\right] .
\end{aligned}
$$

Os resíduos possuem a seguinte estrutura

$$
\text { Resíduo }_{1}=A_{1}+A_{2} p_{0}^{2}+A_{3} \mathbf{p}^{2}+A_{4}\left(\mathbf{p}^{2}\right)^{2}
$$

$\mathrm{e}$

$$
\operatorname{Resíduo}_{2}=\widetilde{A}_{1}
$$

Os valores das constantes $A_{i}$ podem ser lidos dos cálculos anteriores:

$$
A_{1}=-\frac{\left(3 b_{\psi}^{4}+12 \alpha_{\psi} b_{\psi}^{2} M+8 \alpha_{\psi}^{2} M^{2}\right)}{64 \pi^{3} \alpha_{\psi}^{5}}, \quad \widetilde{A}_{1}=-i \frac{\left(-3 b_{\varphi}^{4}+4 \alpha_{\varphi}^{2} m^{2}\right)}{2048 \pi^{3}} \frac{1}{\alpha_{\varphi}^{5}}
$$

$\mathrm{e}$

$$
A_{2}=-\frac{1}{32 \pi^{3}} \frac{1}{\alpha_{\psi}^{3}}, \quad A_{3}=-\frac{1}{16 \pi^{3}} \frac{M}{\alpha_{\psi}^{2}}, \quad A_{4}=-\frac{1}{128 \pi^{3}} \frac{1}{\alpha_{\psi}} .
$$




\section{A.2.2 Função de 2 pontos espinorial}

A função de dois pontos espinorial renormalizada, $N_{B}=0$ e $N_{F}=2$, é

$$
\Gamma_{\psi}^{(2)}(p)=i\left[\gamma^{0} p^{0}-b_{\psi} \gamma \cdot \mathbf{p}-\alpha_{\psi} \mathbf{p}^{2}-M+g^{2}\left(\text { Parte finita }_{3}-\ln \mu \text { Resíduo }_{3}\right)\right]
$$

com o resíduo

$$
\text { Resíduo }_{3}=B_{1}+B_{2} \gamma^{0} p^{0}+B_{3} \gamma \cdot \mathbf{p}+B_{4} \mathbf{p}^{2} .
$$

Os valores das constantes $B_{i}$ são dados por

$$
\begin{gathered}
B_{1}=-\frac{1}{256 \pi^{3} \alpha_{\varphi}^{3} \alpha_{\psi}^{2}\left(\alpha_{\varphi}^{2}-\alpha_{\psi}^{2}\right)^{2}} \\
\times\left[\alpha_{\psi}^{5} b_{\varphi}^{2}+\alpha_{\varphi}^{2} \alpha_{\psi}^{2}\left[\left(2 \alpha_{\varphi}-3 \alpha_{\psi}\right) b_{\varphi}^{2}+\left(-3 \alpha_{\varphi}+2 \alpha_{\psi}\right) b_{\psi}^{2}+2 \alpha_{\psi}\left(-2 \alpha_{\varphi}+\alpha_{\psi}\right) M\right)\right] \\
\left.+\alpha_{\varphi}^{4}\left(\alpha_{\varphi} b_{\psi}^{2}+2 \alpha_{\psi}^{2} M\right)\right] \\
B_{2}=-\frac{1}{128 \pi^{3}} \frac{1}{\alpha_{\varphi}\left(\alpha_{\varphi}+\alpha_{\psi}\right)^{2}} \\
B_{3}=\frac{1}{384 \pi^{3}} \frac{b_{\psi}}{\alpha_{\varphi} \alpha_{\psi}} \frac{\left(2 \alpha_{\varphi}+\alpha_{\psi}\right)}{\left(\alpha_{\varphi}+\alpha_{\psi}\right)^{2}} \\
B_{4}=-\frac{1}{384 \pi^{3}} \frac{\alpha_{\psi}}{\alpha_{\varphi}} \frac{\left(3 \alpha_{\varphi}+\alpha_{\psi}\right)}{\left(\alpha_{\varphi}+\alpha_{\psi}\right)^{3}}
\end{gathered}
$$

e

\section{A.2.3 Função de 3 pontos}

A função de 3 pontos renormalizada, $N_{B}=1$ e $N_{F}=2$, é dada por

$$
\Gamma^{(3)}=-g \Gamma^{5}-g^{3}\left(\text { Parte finita }_{4}-\ln \mu \text { Resíduo }_{4}\right),
$$

com o resíduo

$$
\text { Resíduo }_{4}=C_{1}=\frac{1}{128 \pi^{3}} \frac{1}{\alpha_{\varphi} \alpha_{\psi}^{2}+\alpha_{\varphi}^{2} \alpha_{\psi}} \Gamma^{5}
$$

\section{A.2.4 Função de 4 pontos}

A função de 4 pontos renormalizada, $N_{B}=4$ e $N_{F}=0$, é $\Gamma^{(4)}=-i \lambda+g^{4}\left(\right.$ Parte finita $_{5}-\ln \mu$ Resíduo $\left._{5}\right)-\lambda^{2}\left(\right.$ Parte finita $_{6}-\ln \mu$ Resíduo $\left._{6}\right)$, 
em que,

$$
\text { Resíduo }_{5}=D_{1}=\frac{3 i}{8 \pi^{3}} \frac{1}{\alpha_{\psi}^{3}}
$$

e

$$
\text { Resíduo }_{6}=\widetilde{D}_{1}=i \frac{3}{512 \pi^{3}} \frac{1}{\alpha_{\varphi}^{3}} .
$$

\section{A.3 Solução Perturbativa}

Vamos considerar expansões de todas as funções envolvidas nas equações do grupo de renormalização em termos das constantes de acoplamento $g$ e $\lambda$. Isso nos levará a um sistema de equações que podem ser resolvidas para determinar os parâmetros efetivos.

\section{A.3.1 Função de 2 pontos escalar}

Para a função de 2 pontos escalar (A.58), obtemos as seguintes relações:

$$
\begin{gathered}
-g^{2} A_{1}-\delta_{m^{2}}\left(g^{2}\right)+2 m^{2} \gamma_{\varphi}\left(g^{2}\right)=0, \\
-\lambda \widetilde{A}_{1}-i \delta_{m^{2}}(\lambda)=0, \\
\gamma_{\varphi}\left(g^{2}\right)=-\frac{A_{2}}{2} g^{2} \\
-g^{2} A_{3}-\beta_{b_{\varphi}^{2}}\left(g^{2}\right)+2 b_{\varphi}^{2} \gamma_{\varphi}\left(g^{2}\right)=0
\end{gathered}
$$

e

$$
-g^{2} A_{4}-\beta_{\alpha_{\varphi}^{2}}\left(g^{2}\right)+2 \alpha_{\varphi}^{2} \gamma_{\varphi}\left(g^{2}\right)=0 .
$$

Os termos entre parênteses denotam a ordem da constante de acolpamento correspondente.

\section{A.3.2 Função de 2 pontos espinorial}

Para a função de 2 pontos espinorial (A.63), encontramos,

$$
\begin{gathered}
-g^{2} B_{1}-\delta_{M}\left(g^{2}\right)+2 M \gamma_{\psi}\left(g^{2}\right)=0, \\
\gamma_{\psi}\left(g^{2}\right)=-\frac{B_{2}}{2} g^{2}, \\
-g^{2} B_{3}-\beta_{b_{\psi}}\left(g^{2}\right)+2 b_{\psi} \gamma_{\psi}\left(g^{2}\right)=0
\end{gathered}
$$

e

$$
-g^{2} B_{4}-\beta_{\alpha_{\psi}}\left(g^{2}\right)+2 \alpha_{\psi} \gamma_{\psi}\left(g^{2}\right)=0 .
$$




\section{A.3.3 Função de 3 pontos}

Para a função de 3 pontos (A.69), temos apenas

$$
C_{1} g^{3}-\beta_{g}\left(g^{3}\right)+g\left(\gamma_{\varphi}\left(g^{2}\right)+2 \gamma_{\psi}\left(g^{2}\right)\right)=0 .
$$

\section{A.3.4 Função de 4 pontos}

Finalmente, para a função de 4 pontos (A.71), obtemos

$$
\begin{gathered}
\beta_{\lambda}\left(g^{4}\right)=i D_{1} g^{4}, \\
\beta_{\lambda}\left(\lambda^{2}\right)=-i \widetilde{D}_{1} \lambda^{2}
\end{gathered}
$$

e

$$
\beta_{\lambda}\left(\lambda g^{2}\right)=4 \lambda \gamma_{\varphi}\left(g^{2}\right)
$$

\section{A.4 Parâmetros Efetivos}

A partir dos resultados anteriores, obtemos as relações desejadas para os parâmetros efetivos de interesse

$$
\begin{gathered}
\beta_{b_{\varphi}^{2}}\left(g^{2}\right)=-\left(b_{\varphi}^{2} A_{2}+A_{3}\right) g^{2}=\frac{1}{32 \pi^{3}} \frac{\left(b_{\varphi}^{2}+2 \alpha_{\psi} M\right)}{\alpha_{\psi}^{3}} g^{2}, \\
\beta_{\alpha_{\varphi}^{2}}\left(g^{2}\right)=-\left(\alpha_{\varphi}^{2} A_{2}+A_{4}\right) g^{2}=\frac{1}{384 \pi^{3}} \frac{\left(12 \alpha_{\varphi}^{2}+3 \alpha_{\psi}^{2}\right)}{\alpha_{\psi}^{3}} g^{2}, \\
\beta_{b_{\psi}}\left(g^{2}\right)=-\left(b_{\psi} B_{2}+B_{3}\right) g^{2}=\frac{b_{\psi}}{192 \pi^{3}} \frac{\left(\alpha_{\varphi}+2 \alpha_{\psi}\right)}{\alpha_{\varphi} \alpha_{\psi}\left(\alpha_{\varphi}+\alpha_{\psi}\right)^{2}} g^{2}, \\
\beta_{\alpha_{\psi}}\left(g^{2}\right)=-\left(\alpha_{\psi} B_{2}+B_{4}\right) g^{2}=\frac{1}{192 \pi^{3}} \frac{\alpha_{\psi}\left(3 \alpha_{\varphi}+2 \alpha_{\psi}\right)}{\alpha_{\varphi}\left(\alpha_{\varphi}+\alpha_{\psi}\right)^{3}} g^{2}, \\
\beta_{g}\left(g^{3}\right)=\left(-\frac{A_{2}}{2}-B_{2}+C_{1}\right) g^{3}=\frac{1}{128 \pi^{3}}\left[\frac{2 \alpha_{\varphi}^{3}+4 \alpha_{\varphi}^{2} \alpha_{\psi}+3 \alpha_{\varphi} \alpha_{\psi}^{2}+2 \alpha_{\psi}^{3}}{\alpha_{\psi}^{3} \alpha_{\varphi}\left(\alpha_{\varphi}+\alpha_{\psi}\right)^{2}}\right] g^{3}
\end{gathered}
$$

e

$$
\beta_{\lambda}=-i \widetilde{D}_{1} \lambda^{2}+i D_{1} g^{4}-2 A_{2} \lambda g^{2}=\frac{3}{512 \pi^{3}} \frac{\lambda^{2}}{\alpha_{\varphi}^{3}}-\frac{3}{8 \pi^{3}} \frac{g^{4}}{\alpha_{\psi}^{3}}+\frac{1}{16 \pi^{3}} \frac{\lambda g^{2}}{\alpha_{\psi}^{3}} \text {. }
$$




\section{Apêndice B}

\section{Teorema de Noether para teorias com derivadas de terceira ordem}

Neste apêndice, apresentamos uma dedução do teorema de Noether para teorias com derivadas de terceira ordem. Vamos supor que o campo básico varia continuamente de $\varphi(x)$ para $\varphi(x)+\delta \varphi$ implicando na seguinte variação para a densidade de lagrangiana

$$
\delta \mathcal{L}=\frac{\partial \mathcal{L}}{\partial \varphi} \delta \varphi+\frac{\partial \mathcal{L}}{\partial \partial_{\mu} \varphi} \delta \partial_{\mu} \varphi+\frac{\partial \mathcal{L}}{\partial \partial_{\mu} \partial_{\nu} \varphi} \delta \partial_{\mu} \partial_{\nu} \varphi+\frac{\partial \mathcal{L}}{\partial \partial_{\mu} \partial_{\nu} \partial_{\rho} \varphi} \delta \partial_{\mu} \partial_{\nu} \partial_{\rho} \varphi
$$

Agora, usando a equação de movimento de Euler-Lagrange,

$$
-\frac{\partial \mathcal{L}}{\partial \varphi}+\partial_{\mu} \frac{\partial \mathcal{L}}{\partial \partial_{\mu} \varphi}-\partial_{\mu} \partial_{\nu} \frac{\partial \mathcal{L}}{\partial \partial_{\mu} \partial_{\nu} \varphi}+\partial_{\mu} \partial_{\nu} \partial_{\rho} \frac{\partial \mathcal{L}}{\partial \partial_{\mu} \partial_{\nu} \partial_{\rho} \varphi}=0
$$

obtemos

$$
\delta \mathcal{L}=\partial_{\mu}\left(\frac{\partial \mathcal{L}}{\partial \partial_{\mu} \varphi} \delta \varphi+\frac{\partial \mathcal{L}}{\partial \partial_{\mu} \partial_{\nu} \varphi} \overleftrightarrow{\partial}_{\nu} \delta \varphi\right)+\left(\partial_{\mu} \partial_{\nu} \partial_{\rho} \frac{\partial \mathcal{L}}{\partial \partial_{\mu} \partial_{\nu} \partial_{\rho} \varphi}\right) \delta \varphi+\frac{\partial \mathcal{L}}{\partial \partial_{\mu} \partial_{\nu} \partial_{\rho} \varphi} \delta \partial_{\mu} \partial_{\nu} \partial_{\rho} \varphi
$$

Ao usar esse resultado em (B.1), podemos escrevê-la como

$$
\begin{aligned}
\delta \mathcal{L} & =\partial_{\mu}\left(\frac{\partial \mathcal{L}}{\partial \partial_{\mu} \varphi} \delta \varphi+\frac{\partial \mathcal{L}}{\partial \partial_{\mu} \partial_{\nu} \varphi} \stackrel{\leftrightarrow}{\partial}_{\nu} \delta \varphi\right)+\partial_{\mu}\left(\partial_{\nu} \partial_{\rho} \frac{\partial \mathcal{L}}{\partial \partial_{\mu} \partial_{\nu} \partial_{\rho} \varphi} \delta \varphi\right) \\
& -\partial_{\nu}\left(\partial_{\rho} \frac{\partial \mathcal{L}}{\partial \partial_{\mu} \partial_{\nu} \partial_{\rho} \varphi} \partial_{\mu} \delta \varphi\right)+\partial_{\rho} \frac{\partial \mathcal{L}}{\partial \partial_{\mu} \partial_{\nu} \partial_{\rho} \varphi} \partial_{\nu} \partial_{\mu} \delta \varphi+\frac{\partial \mathcal{L}}{\partial \partial_{\mu} \partial_{\nu} \partial_{\rho} \varphi} \delta \partial_{\mu} \partial_{\nu} \partial_{\rho} \varphi
\end{aligned}
$$

em que $A \overleftrightarrow{\partial}_{\mu} B \equiv A \partial_{\mu} B-\left(\partial_{\mu} A\right) B$. Por outro lado, sem empregar a equação de movimento, se a variação da lagrangiana for uma derivada total $\delta \mathcal{L}=\partial_{\mu} S^{\mu}$, podemos então definir uma corrente conservada por

$$
J^{\mu} \equiv \frac{\partial \mathcal{L}}{\partial \partial_{\mu} \varphi} \delta \varphi+\left(\frac{\partial \mathcal{L}}{\partial \partial_{\mu} \partial_{\nu} \varphi}-\partial_{\rho} \frac{\partial \mathcal{L}}{\partial \partial_{\mu} \partial_{\nu} \partial_{\rho} \varphi}\right) \stackrel{\leftrightarrow}{\partial} \delta \varphi+\frac{\partial \mathcal{L}}{\partial \partial_{\mu} \partial_{\nu} \partial_{\rho} \varphi} \partial_{\nu} \partial_{\rho} \delta \varphi-S^{\mu}
$$


Apêndice B. Teorema de Noether para teorias com derivadas de terceira ordem

Esse resultado pode ser facilmente generalizado. Se a lagrangiana contem derivadas de quarta ordem, um procedimento semelhante mostra que a corrente conservada é dada por

$$
\begin{aligned}
J^{\mu}= & \frac{\partial \mathcal{L}}{\partial \partial_{\mu} \varphi} \delta \varphi+\left(\frac{\partial \mathcal{L}}{\partial \partial_{\mu} \partial_{\nu} \varphi}-\partial_{\rho} \frac{\partial \mathcal{L}}{\partial \partial_{\mu} \partial_{\nu} \partial_{\rho} \varphi}+\partial_{\rho} \partial_{\sigma} \frac{\partial \mathcal{L}}{\partial \partial_{\mu} \partial_{\nu} \partial_{\rho} \partial_{\sigma} \varphi}\right) \stackrel{\leftrightarrow}{\partial_{\nu}} \delta \varphi+\frac{\partial \mathcal{L}}{\partial \partial_{\mu} \partial_{\nu} \partial_{\rho} \varphi} \partial_{\nu} \partial_{\rho} \delta \varphi \\
& +\frac{\partial \mathcal{L}}{\partial \partial_{\mu} \partial_{\nu} \partial_{\rho} \partial_{\sigma} \varphi} \stackrel{\leftrightarrow}{\partial}_{\nu}\left(\partial_{\rho} \partial_{\sigma} \delta \varphi\right)-S^{\mu} .
\end{aligned}
$$




\section{Apêndice $\mathrm{C}$}

\section{Soma de Séries Infinitas via Teorema de Resíduos}

Queremos avaliar uma somatória do tipo:

$$
\sum_{n=-\infty}^{+\infty} \frac{1}{n^{2}+y^{2}}
$$

Nossa estratégia para este fim, trata-se essencialmente da utilização do teorema dos resíduos, como passamos a descrever. Consideremos uma função $g(z)$ composta pelo produto de uma função $f(z)$, que não tenha pólos sobre o eixo imaginário, pela função $\operatorname{coth}\left(\frac{\beta z}{2}\right)$, ou seja,

$$
g(z) \equiv f(z) \operatorname{coth}\left(\frac{\beta z}{2}\right)
$$

Notemos que os pólos de $g(z)$ coincidem com os de $\operatorname{coth}\left(\frac{\beta z}{2}\right)$ e estão lozalizados em $z=2 \pi n i / \beta$. Consideremos agora a integral no plano complexo de $g(z)$ com o contorno $C=\bigcup_{i} C_{i}$ mostrado na figura (C.1),

$$
I \equiv \oint_{C} d z f(z) \operatorname{coth}\left(\frac{\beta z}{2}\right) .
$$

Então, de acordo com o teorema dos resíduos,

$$
\oint_{C} d z f(z)=2 \pi i \sum \operatorname{Res} f(z)
$$




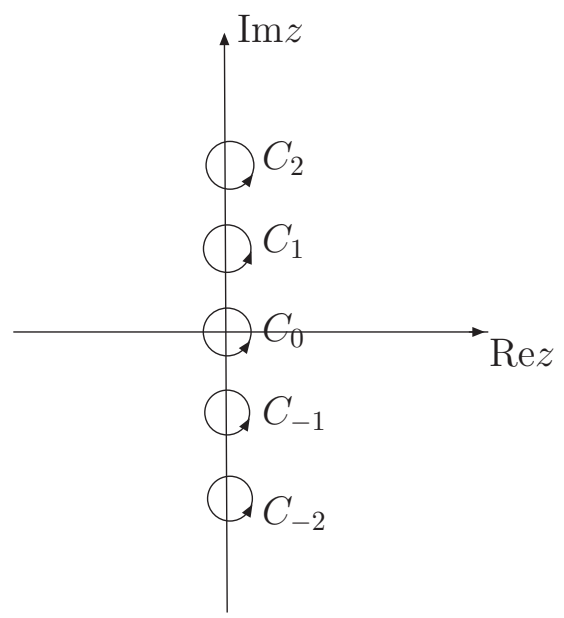

Figura C.1: Contorno $C=\bigcup_{i} C_{i}$ no plano complexo de $z$.

a expressão (C.3) torna-se

$$
\begin{aligned}
I & =2 \pi i \sum_{n=-\infty}^{+\infty} \operatorname{Res}_{z_{0}=\frac{2 \pi n i}{\beta}} f\left(z_{0}\right) \operatorname{coth}\left(\frac{\beta z_{0}}{2}\right) \\
& =2 \pi i \sum_{n=-\infty}^{+\infty} \lim _{z_{0} \rightarrow \frac{2 \pi n i}{\beta}}\left(z_{0}-\frac{2 \pi n i}{\beta}\right) f\left(z_{0}\right) \operatorname{coth}\left(\frac{\beta z_{0}}{2}\right) \\
& =4 \pi i \sum_{n=-\infty}^{+\infty} \frac{1}{\beta} f\left(\frac{2 \pi n i}{\beta}\right),
\end{aligned}
$$

ou ainda,

$$
\sum_{n=-\infty}^{+\infty} \frac{1}{\beta} f\left(\frac{2 \pi n i}{\beta}\right)=\frac{1}{4 \pi i} I .
$$

Por outro lado, podemos escrever a integral de contorno $I$ em (C.3) de outra maneira deformando o contorno $C$ original como indicado na figura (‥2). Dessa modo, $I$ tornase:

$$
I=\int_{-i \infty+\epsilon}^{i \infty+\epsilon} d z f(z) \operatorname{coth}\left(\frac{\beta z}{2}\right)+\int_{i \infty-\epsilon}^{-i \infty-\epsilon} d z f(z) \operatorname{coth}\left(\frac{\beta z}{2}\right) .
$$

A expressão (C.7) pode facilmente ser separada em uma parte dependente da temperatura e uma independente, tal que podemos escrever

$$
\sum_{n=-\infty}^{+\infty} \frac{1}{\beta} f\left(\frac{2 \pi n i}{\beta}\right)=\frac{1}{2 \pi i} \int_{-i \infty+\epsilon}^{i \infty+\epsilon} d z[f(z)+f(-z)] n_{B}(z)+\frac{1}{4 \pi i} \int_{i \infty}^{-i \infty} d z[f(z)+f(-z)] .
$$




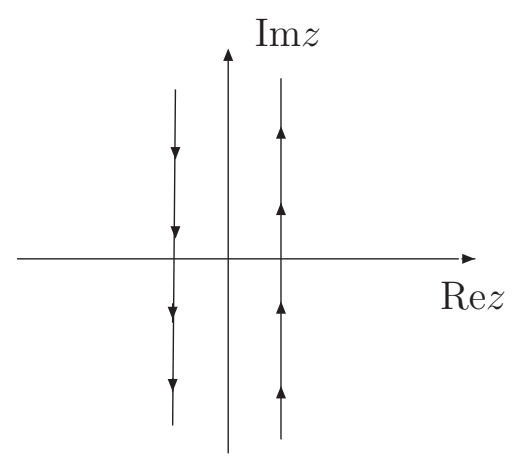

Figura C.2: Contorno deformado.

Esse é nosso resultado final. Ele nos diz como relacionar uma soma infinita com integrais no plano complexo, que frequentemente podem ser calculadas.

Vamos aplicar (C.8) para o cálculo da série (C.1). Nesse caso, devemos identificar

$$
f\left(\frac{2 \pi n i}{\beta}\right) \equiv \frac{\beta}{n^{2}+y^{2}} .
$$

Fazendo $z \equiv \frac{2 \pi n i}{\beta}$, obtemos

$$
f(z)=\frac{(2 \pi i)^{2}}{\beta} \frac{1}{z^{2}-\left(\frac{2 \pi y}{\beta}\right)^{2}}
$$

Note que os pólos de $\mathrm{f}(\mathrm{z})$ estão localizados sobre o eixo real, tal que o desenvolvimento acima podem ser usados sem dificuldades. De acordo com (C.8), segue que

$$
\sum_{n=-\infty}^{+\infty} \frac{1}{n^{2}+y^{2}}=\frac{4 \pi i}{\beta} \int_{-i \infty+\epsilon}^{i \infty+\epsilon} d z \frac{1}{z^{2}-\left(\frac{2 \pi y}{\beta}\right)^{2}} \frac{1}{e^{\beta z}-1}+\frac{2 \pi i}{\beta} \int_{i \infty}^{-i \infty} d z \frac{1}{z^{2}-\left(\frac{2 \pi y}{\beta}\right)^{2}}
$$

Basta calcular as duas integrais do lado direito. Para calcular a primeira delas consideremos a seguinte integral sobre o contorno $C_{1}$ mostrado na figura (C.3),

$$
\oint_{C_{1}} d z \frac{1}{z^{2}-\left(\frac{2 \pi y}{\beta}\right)^{2}} \frac{1}{e^{\beta z}-1}
$$

Pelo teorema dos rezíduos, temos

$$
\oint_{C_{1}} d z \frac{1}{z^{2}-\left(\frac{2 \pi y}{\beta}\right)^{2}} \frac{1}{e^{\beta z}-1}=-\frac{i \beta}{2 y} \frac{1}{e^{2 \pi y}-1}
$$




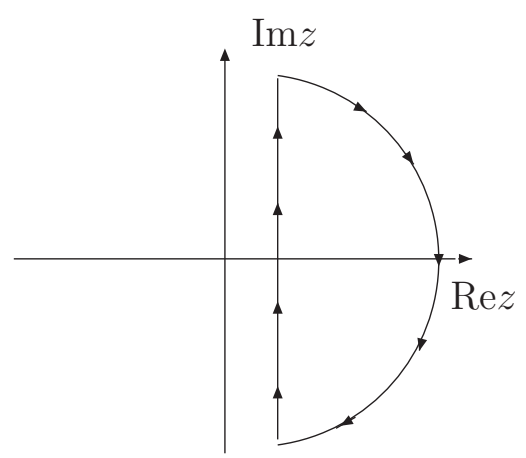

Figura C.3: Contorno $C_{1}$.

O sinal negativo vem do fato que o sentido adotado para o caminho $C_{1}$ é oposto àquele definido nas figuras (C.1) e (C.2). Observe também que o integrando acima tem dois pólos (sobre o eixo real); um dentro do contorno $C_{1}$, fornecendo a contribuição não nula, e o outro pólo fora do contorno. O lado esquerdo de (C.13) pode ser decomposto em uma soma sobre os dois caminhos que compõe o contorno $C_{1}$, quer dizer,

$$
\oint_{C_{1}} d z \frac{1}{z^{2}-\left(\frac{2 \pi y}{\beta}\right)^{2}} \frac{1}{e^{\beta z}-1}=\int_{-i \infty+\epsilon}^{i \infty+\epsilon} d z \frac{1}{z^{2}-\left(\frac{2 \pi y}{\beta}\right)^{2}} \frac{1}{e^{\beta z}-1}+\lim _{R \rightarrow \infty} \int_{R} d z \frac{1}{z^{2}-\left(\frac{2 \pi y}{\beta}\right)^{2}} \frac{1}{e^{\beta z}-1} .
$$

A segunda integral do lado é direito é nula. Para ver isso, basta fazer a parametrização $z=R e^{i \theta}$. Dessa forma,

$$
\lim _{R \rightarrow \infty} \int_{\frac{\pi}{2}}^{-\frac{\pi}{2}} d \theta \frac{i e^{i \theta}}{R-\frac{1}{R}\left(\frac{2 \pi y}{\beta}\right)^{2}} \frac{1}{e^{\beta R(\cos \theta+i \sin \theta)}-1}=0
$$

Portanto, estabelecemos o resultado desejado

$$
\int_{-i \infty+\epsilon}^{i \infty+\epsilon} d z \frac{1}{z^{2}-\left(\frac{2 \pi y}{\beta}\right)^{2}} \frac{1}{e^{\beta z}-1}=-\frac{i \beta}{2 y} \frac{1}{e^{2 \pi y}-1}
$$

A segunda integral em (C.11) pode ser calculada da mesma forma. Consideremos a integral sobre o contorno $C_{2}$ indicado na figura (C.4),

$$
\oint_{C_{2}} d z \frac{1}{z^{2}-\left(\frac{2 \pi y}{\beta}\right)^{2}}
$$

De acordo com o teorema dos rezíduos, temos que 


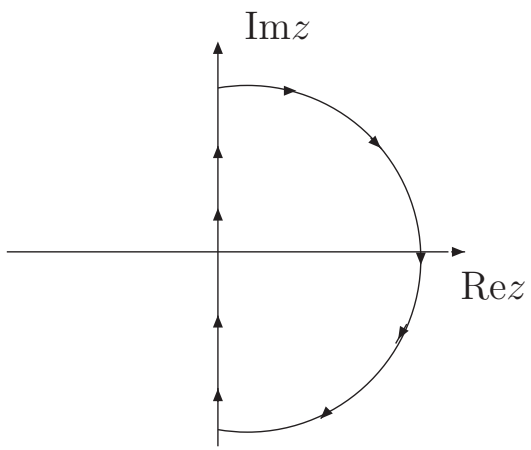

Figura C.4: Contorno $C_{2}$.

$$
\oint_{C_{2}} d z \frac{1}{z^{2}-\left(\frac{2 \pi y}{\beta}\right)^{2}}=-\frac{i \beta}{2 y} .
$$

Decompondo a integral sobre o caminho $C_{2}$ nas duas partes que o compõe e observando que apenas a parte sobre o eixo imaginário contribui, concluímos que

$$
\int_{-i \infty}^{i \infty} d z \frac{1}{z^{2}-\left(\frac{2 \pi y}{\beta}\right)^{2}}=-\frac{i \beta}{2 y} .
$$

Finalmente, substituindo (C.16) e (C.19) em (C.11) obtemos o resultado

$$
\sum_{n=-\infty}^{+\infty} \frac{1}{n^{2}+y^{2}}=\frac{\pi}{y} \operatorname{coth}(\pi y)
$$




\section{Referências Bibliográficas}

[1] G. t'Hooft, M. Veltman, One loop divergencies in the theory of gravitation, Ann. Inst Henri Poincare, 20, 69-94, (1974).

[2] J. Polchinski, String theory. An Introduction to the Bosonic String. Vol.1, Cambridge University Press, Cambridge, 1998.

[3] S. Chadha, H. B. Nielsen, Lorentz Invariance As A Low-energy Phenomenon, Nucl. Phys. B 217, 125-144, (1983).

[4] S. Carroll, G. Field, R. Jackiw, Limits on a Lorentz and Parity Violating Modification of Electrodynamics, Phys. Rev. D 41, 1231, (1990).

[5] V. A. Kostelecky, Gravity, Lorentz violation, and the standard model, Phys. Rev. D 69, 105009, (2004), hep-th/0312310.

[6] M. Gomes, J.R. Nascimento, A. Yu Petrov, A. J. Silva, On the aether-like Lorentzbreaking actions, Phys. Rev. D, 045018, (2010), arXiv:0911.3548.

[7] T. Jacobson, S. Liberati, D. Mattingly, Lorentz violation at high energy: Concepts, phenomena and astrophysical constraints, Ann. Phys. 321, 150, (2006), astro$\mathrm{ph} / 0505267$.

[8] S. W. Hawking, T. Hertog, Living with ghosts, Phys. Rev. D 65, 103515, (2002), hep-th/0107088.

[9] I. Antoniadis, E. Dudas, D. M. Ghilencea, Living with ghosts and their radiative corrections, Nucl. Phys. B 767, 29-53, (2007), hep-th/0608094.

[10] D. Anselmi, M. Halat, Renormalization of Lorentz violating theories, Phys. Rev. D 76, 125011, (2007), arXiv:0707.2480.

[11] D. Anselmi, Weighted scale invariant quantum field theories, JHEP 0802, 051, (2008), arXiv:0801.1216.

[12] P. Horava, Membranes at Quantum Criticality, JHEP 0903, 020, (2009), arXiv:0812.4287. 
[13] P. Horava, Quantum Gravity at a Lifshitz Point, Phys. Rev. D 79, 084008, (2009), arXiv:0901.3775.

[14] T. Vojta, Quantum phase transitions, em K.H. Hoffmann e M. Schreiber (Eds): Computational Statistical Physics, Springer, Berlin (2002), cond-mat/0010285.

[15] T. Vojta, Quantum phase transitions, Rep. Prog. Phys. 66, 2069, (2003), condmat/0309604.

[16] I. Herbut, A Modern Approach to Critical Phenomena, Cambridge University Press, Cambridge, 2007.

[17] R. M. Hornreich, The Lifshitz Point: Phase Diagrams and Critical Behavior, J. Magn. Magn. Mater. 15-18, 387, (1980).

[18] K.G. Wilson, and J. B. Kogut, The Renormalization group and the epsilon expansion, Phys. Rept. 12: 75-200, (1974).

[19] D. J. Amit, Field Theory, the Renormalization Group, and Critical Phenomena, World Scientific, Singapore, 1984.

[20] J. Z. Justin, Quantum Field Theory and Critical Phenomena, Clarendon Press Oxford, 1996.

[21] T. H. Berlin and M. Kac, The Spherical Model of a Ferromagnet, Phys. Rev. 86, 821 (1952).

[22] H. E. Stanley, Spherical Model as the Limit of Infinite Spin Dimensionality, Phys. Rev. 176, 718, (1968).

[23] G. Obermair, Dynamical Aspects of Critical Phenomena, edited by J. I. Budnick and M. P. Kawara (Gordon and Breach, New York, 1972), p. 137.

[24] Th. M. Nieuwenhuizen, Quantum description of spherical spins, Phys. Rev. Lett. 74, 4293, (1995), arXiv:cond-mat/9408055.

[25] S. Sachdev, Quantum Phase Transitions, Cambridge University Press, New York, 2006.

[26] T. Vojta, Quantum version of a spherical model: Crossover from quantum to classical critical behavior, Phys. Rev. B 53, 710, (1996).

[27] H. Nicolai, Supersymmetry and spin systems, J. Phys. A: Math. Gen., Vol. 9, 1497, (1976). 
[28] H. Nicolai, Extensions of supersymmetric spin systems, J. Phys. A: Math. Gen., Vol. 10, 2143, (1977).

[29] E. Witten, Dynamical Breaking of Supersymmetry, Nucl. Phys. B 188, 513 (1981).

[30] G. Parisi and N. Sourlas, Random Magnetic Fields, Supersymmetry and Negative Dimensions, Phys. Rev. Lett. 43, 744, (1979).

[31] N. Sourlas, Introduction to supersymmetry in condensed matter physics, Physica 15 D, 115-122, (1985).

[32] G. Junker, Supersymmetric Methods in Quantum and Statistical Physics, SpringerVerlag Berlin Heidelberg New York, 1995.

[33] K. S. Stelle, Renormalization of Higher Derivative Quantum Gravity, Phys. Rev. D 16, 953-969, (1977).

[34] R. Iengo, J. G. Russo e M. Serone, Renormalization group in Lifshitz-type theories, JHEP 0911, 020 (2009), arXiv:0906.3477.

[35] R. Iengo e M. Serone, A Simple UV-Completion of QED in 5D, Phys. Rev. D81, 125005 (2010), arXiv:1003.4430.

[36] J. J. Binney, N. J. Dowrick, A. J. Fisher, M. E. J. Newman, The Theory of Critical Phenomena, Oxford Univ. Press, New York, 1992.

[37] P. R. S. Gomes, Uma Introdução a Fenômenos Críticos via Teoria de Perturbação, Trabalho de Graduação, Departamento de Física, UEM, 2006.

[38] P. F. Bienzobaz e S. R. Salinas, Quantum spherical model with competing interactions, Physica A, 391, 6399, (2012), arXiv:1203.4073.

[39] P. Horava, Quantum Criticality and Yang-Mills Gauge Theory, Phys. Lett. B 694, 172-176, (2010), arXiv:0811.2217.

[40] R. L. Arnowitt, S. Deser e C. W. Misner, The Dynamics of general relativity, grqc/0405109.

[41] R. M. Wald, General Relativity, The University of Chicago Press, Chicago, 1984.

[42] P. R. S. Gomes e M. Gomes, On Higher Spatial Derivative Field Theories, Phys. Rev. D 85, 085018, (2012), arXiv:1107.6040.

[43] T. Muta, Foundations of Quantum Chromodynamics, World Scientific, Singapore, 2010. 
[44] J. Collins, Renormalization, Cambridge University Press, New York, 1984.

[45] M. Gomes, Teoria Quântica dos Campos, Editora da Universidade de São Paulo, São Paulo, 2002.

[46] C. Mergulhão, C. E. I. Carneiro, Field-theoretic calculation of critical exponents for the Lifshitz point, Phys. Rev. B 59, 13954, (1998).

[47] P. R. S. Gomes e M. Gomes, On Ward Identities in Lifshitz-like Field Theories, Phys. Rev. D 85, 065010, (2012), arXiv:1112.3887.

[48] S. Pokorski, Gauge Field Theories, Cambridge University Press, 2000.

[49] K. Fujikawa e H. Suzuki, Path Integral and Quantum Anomalies, Oxford University Press, 2004.

[50] W. Zimmermann, W. Zimmermann, em Lectures on Elementary Particles and Quantum Field Theory, edited by S. Deser, M. Grisaru, and H. Pendleton (MIT Press, Cambridge, 1970), 397.

[51] J. H. Lowenstein, Normal product quantization of currents in Lagrangian field theory, Phys. Rev.D 4 2281, (1971).

[52] I. Adam, I. V. Melnikov and S. Theisen, A Non-Relativistc Weyl Anomaly, JHEP 0909:130, 2009, arXiv:0907.2156.

[53] I. Bakas and D. Lust, Axial anomalies of Lifshitz fermions, Fortschr. Phys. 59 (2011) 937, hep-th/1103.5693.

[54] I. Bakas, More on axial anomalies of Lifshitz fermions, hep-th/1110.1332.

[55] M. Baggio, J. de Boer e K. Holsheimer, Anomalous Breaking of Anisotropic Scaling Symmetry in the Quantum Lifshitz Model, JHEP 1207, 099, (2012), arXiv:1112.6416.

[56] G. Mussardo, Statistical Field Theory, Oxford University Press, New York, 2010.

[57] G. S. Joyce, Phase Transitions and Critical Phenomena, edited by C. Domb and M. Grenn, Vol. 2, Academic Press, New York, (1972).

[58] P. F. Bienzobaz, Quantização Canônica e Integração Funcional no Modelo Esférico Médio, Tese de doutorado, IFUSP, 2012.

[59] P. R. S. Gomes, P. F. Bienzobaz e M. Gomes, Supersymmetic Extension of the Quantum Spherical Model, Phys. Rev. E 85, 061109, (2012), arXiv:1203.5074.

[60] M. H. Oliveira, E. P. Raposo, e M. D. Coutinho Filho, Quantum spherical spin model on hypercubic lattices, Phys. Rev. B 74, 184101, (2006). 
[61] A. A. Abrikosov, L. P. Gorkov e I. E. Dzyaloshinski, Methods of Quantum Field Theory in Statistical Physics, Pergamon Press, New York, 1965.

[62] J. I. Kapusta, Finite-Temperature Field Theory, Cambridge University Press, New York, 1989.

[63] A. Das, Finite Temperature Field Theory, World Scientific, Singapore, 1997.

[64] S. Weinberg, The Quantum Theory of Fields, Vol.3, Supersymmetry, Cambridge University Press, New York, 2010.

[65] A. Das e M. Kaku, Supersymmetry At High Temperatures, Phys. Rev. D 18, 4540, (1978).

[66] H. Matsumoto, M. Nakahara, Y. Nakano and H. Umezawa, Supersymmetry at finite temperature, Phys. Rev. D 29, 28382850 (1984).

[67] E. Witten, Constraints on Supersymmetry Breaking, Nucl. Phys. B 202, 253, (1982).

[68] A. Das, A. Kharev e V. S. Mathur, The Witten Index at Finite Temperature, Phys. Lett. B181, 299, (1986).

[69] G. W. Semenoff e H. Umezawa, Functional Methods In Thermo Field Dynamics: A Real Time Perturbation Theory For Quantum Statistical Mechanics, Nucl. Phys. B 220, 196-212, (1983).

[70] P.F. Bienzobaz, P. R.S. Gomes e M. Gomes, Stochastic Quantization of the Spherical Model and Supersymmetry, arXiv:1211.5081.

[71] W. Siegel, Supersymmetric dimensional regularization via dimensional reduction, Phys. Lett. 84 B, 193, (1979).

[72] S. J. Gates, M. T. Grisaru, M. Rocek e W. Siegel, Superspace or One thousand and one lessons in supersymmetry, Front. Phys. 58, 1-548, (1983), hep-th/0108200. 